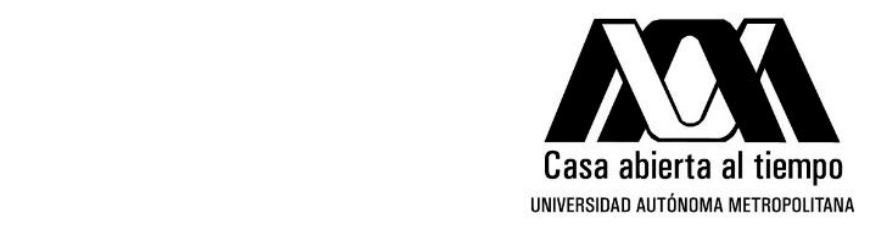

UNIVERSIDAD AUTÓNOMA METROPOLITANA - IZTAPALAPA

DIVISIÓN DE CIENCIAS BÁSICAS E INGENIERÍA

\title{
TRANSFORMADAS NUMÉRICAS APLICADAS A SISTEMAS CUÁNTICOS
}

\author{
Tesis que presenta \\ Saúl Juan Carlos Salazar Samaniego \\ Para obtener el grado de \\ Maestro en Ciencias (Química)
}

Asesor: $\quad$ Dr. Robin Preenja Sagar

Jurado calificador:

Presidente: Dr. José Luis Gázquez Mateos

Secretario: $\quad$ Dr. Marcelo Enrique Galván Espinosa

Vocal:

Dr. Jesús Hernández Trujillo 


\section{AGRADECIMIENTOS}

Quiero agradecer a mi madre y hermana por apoyarme en este camino ustedes son esa luz que me ilumina. Deseando ser un buen ejemplo para mi sobrina.

A mi novia por siempre hecharme porritas tu compañía es el mejor regalo.

A mi asesor el Dr. Robin P. Sagar por su enorme paciencia y disposcición para discutir siempre las ideas con un café. Su guía y consejo enriquecen mi formación.

A mi amigo el Dr. Humberto Laguna por sus siempre acertados comentarios y sus amenas charlas.

A los sinodales por su disposición y tiempo que llevaron en la revisión de este trabajo así como por las observaciones que ayudaron a mejorarlo.

Probamos por medio de la lógica, pero descubrimos por medio de la intuición.

H. Poincaré

Dijo Tennyson que si pudiéramos comprender una sola flor sabríamos quiénes somos y qué es el mundo.

J. L. Borges 


\section{Índice}

1 Introducción 4

2 Espacio de momentos $\quad 6$

2.1 ¿Por qué estudiar el espacio de momentos? . . . . . . . . . 6

2.2 Representación en el espacio de momentos . . . . . . . . . 8

3 Transformada de Fourier $\quad 11$

3.1 Transformada de Fourier en una dimensión . . . . . . . . . . . 12

3.2 Transformada de Fourier en dos dimensiones . . . . . . . . . . 12

3.2.1 Transformada de Hankel . . . . . . . . . . . . . . 12

3.2.1.1 Simetría circular . . . . . . . . . . 13

3.3 Transformada de Fourier en tres dimensiones . . . . . . . . . . 14

3.3.1 Transformada de Bessel esférica . . . . . . . . . 15

3.4 Transformada de Fourier en $n$-dimensiones . . . . . . . . . . 15

3.5 Transformada de Dirac-Fourier de funciones de onda en el espacio de posición . . . . . . . . . . . 16

3.6 Transformada de Dirac-Fourier de orbitales atómicos . . . . . 17

4 Aproximaciones numéricas $\quad 19$

4.1 Cuadratura Gaussiana . . . . . . . . . . . . . . 20

4.2 Polinomios ortogonales . . . . . . . . . . . . . . 21

4.3 Fórmulas de cuadratura Gaussiana y polinomios ortogonales . 21

4.4 Cálculo numérico de integrales con funciones de Bessel y cuadratura Gaussiana en el plano complejo . . . . . . . . . . 24

4.5 Aplicación de la metodología . . . . . . . . . . . . 28

$\begin{array}{llr}5 & \text { Objetivos y alcance } & 30\end{array}$

6 Transformada de Hankel $\quad 31$

6.1 Átomo de hidrógeno en dos dimensiones . . . . . . . . . . 31

6.2 Oscilador armónico en dos dimensiones . . . . . . . . . . . 34

6.3 Discusión de resultados . . . . . . . . . . . . . . . . . . 34

6.3.1 Átomo de hidrógeno en dos dimensiones . . . . . . . . 34

6.3.1.1 $m=0 \ldots \ldots \ldots \ldots \ldots$

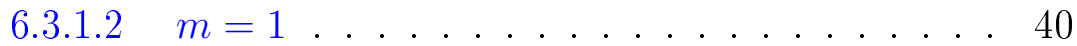

6.3.1.3 $m=2,3 \ldots \ldots \ldots \ldots 42$

6.3.2 Comportamiento del método para valores de $Z>1$. . 44

6.4 Oscilador armónico en dos dimensiones . . . . . . . . . . 45

6.5 Comparación con otra metodología . . . . . . . . . 47 
6.6 Conclusiones ........................ 51

7 Transformada de Bessel esférica $\quad 52$

7.1 Funciones de base tipo Slater . . . . . . . . . . . . . 52

7.1 .1 Desarrollo .................... 53

7.1 .2 Procedimiento . . . . . . . . . . 57

7.2 Funciones de base tipo Gaussianas . . . . . . . . . . . . 58

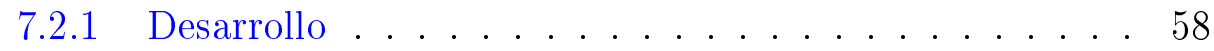

7.2.2 Procedimiento................... 63

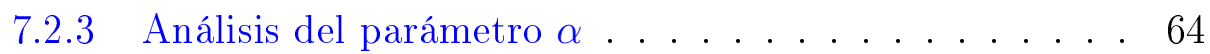

7.2.3.1 $\quad \alpha<1 \ldots \ldots \ldots . \ldots 65$

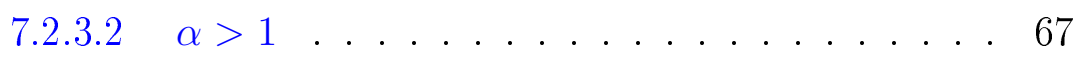

7.2.3.3 Análisis de las sucesiones generadas en el plano complejo al evaluar el método . . . . . 71

7.3 Aplicación a sistemas atómicos: extensión a orbitales atómicos 75

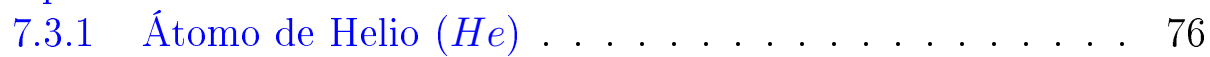

7.3 .2 Átomo de Neón $(\mathrm{Ne}) \ldots \ldots \ldots \ldots$. . . . . 76

7.3 .3 Átomo de Hierro $(F e) \ldots \ldots . \ldots 78$

7.4 Discusión de resultados . . . . . . . . . . . . 78

7.4.1 Funciones de base tipo Slater . . . . . . . . . 78

7.4.1.1 $l=0 \ldots \ldots \ldots \ldots . \ldots \ldots$

$7.4 .1 .2 \quad l=1 \ldots \ldots \ldots . \ldots \ldots 8$

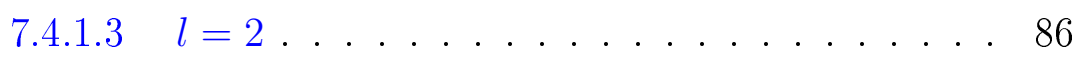

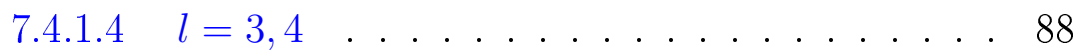

7.4.2 Funciones de base tipo Gaussianas . . . . . . . . 90

7.4.2.1 $l=0 \ldots \ldots \ldots \ldots . \ldots . \ldots 90$

$7.4 .2 .2 \quad l=1 \ldots \ldots \ldots \ldots 2 \ldots \ldots 9 . \ldots \ldots$

7.4.2.3 $\quad l=2,3 \ldots \ldots \ldots . \ldots . \ldots 93$

7.4.2.4 Gaussiana sin transformar . . . . . . . . 95

7.4.3 Aplicación a sistemas atómicos: extensión a orbitales . 96

7.4.3.1 Átomo de Helio $(\mathrm{He}) \ldots . . . . . . .996$

7.4.3.2 Átomo de Neon $(N e) \ldots \ldots . . . . . . . . . .997$

7.4.3.3 Átomo de Hierro $(\mathrm{Fe})$. . . . . . . . . . 101

7.5 Extensión de la metodología para las funciones de base tipo

Slater usando el parámetro $\alpha$. . . . . . . . . . . 102

7.6 Comparación con otra metodología . . . . . . . . . . . . 104

7.6.1 Funciones de base tipo Slater . . . . . . . . . . . . . . 104

7.6.2 Funciones de base tipo Gaussianas . . . . . . . . . . 105

7.7 Conclusiones . . . . . . . . . . . . . . 107

8 Conclusiones generales 109 
10 Apéndice A

10.1 Coeficientes de recurrencia y cuadraturas Gaussianas para funciones de peso $w(x)=x^{m} K_{m}(x)$ para $m=0, m=1$,

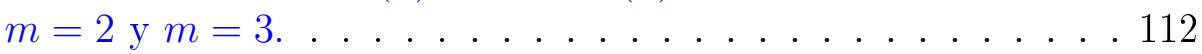

11 Apéndice B

11.1 Gráficas de polinomios ortogonales para $w(x)=x^{m} K_{m}(x)$. . 124

12 Apéndice $\mathrm{C}$

12.1 Coeficientes de recurrencia y cuadraturas Gaussianas para funciones de peso $w(x)=e^{-x} \Theta_{l}(x)$ para $l=0, l=1, l=2 \mathrm{y}$ $l=3$ con el polinomio de Bessel inverso $\Theta_{l}(x) \ldots \ldots . . . .125$

13 Apéndice D

13.1 Gráficas de polinomios ortogonales para $w(x)=e^{-x} \Theta_{l}(x)$. . 150

14 Apéndice E

14.1 Gráficas de las funciones de peso $w(x)=x^{m} K_{m}(x)$. . . . . 151

14.2 Gráficas de las funciones de peso $w(x)=e^{-x} \Theta_{l}(x)$. . . . . 152

15 Apéndice $\mathrm{F}$

16 Bibliografía 


\section{Introducción}

Los métodos de la física matemática han sido fundamentales para el desarrollo de la mecánica cuántica. Desde el origen de esta ciencia la herramienta matemática contribuyó a entender los principios que gobiernan las leyes de las partículas atómicas así como su funcionamiento y lógica.

El auge de la química cuántica en aplicaciones que involucran la interacción entre varias partículas exige el uso de métodos aproximados o numéricos. La importancia de calcular numéricamente integrales viene del hecho de que con frecuencia no se cuenta con las funciones explícitas o analíticas ya que sólo se cuenta con datos numéricos o experimentales.

La correlación entre las variables posición $x$ y momento $p$ que aparece de manera natural en la mecánica cuántica y su relación con el principio de incertidumbre de Heisenberg explican la existencia de una transformación fundamental entre ambos espacios. En este punto radica la importancia de estudiar la información que cada espacio puede capturar de un sistema químico debido a su reciprocidad.

El estudio de modelos atómicos en tres dimensiones fue en un inicio el tema principal de la química cuántica. Posteriormente el desarrollo de modelos atómicos en dos dimensiones se convirtió en un asunto de interés por su aplicación en áreas como la denominada química plana, nanotecnología en 2D, grafeno, entre otras.

En este trabajo se estudia y explora la transformación al espacio de momentos mediante la transformada de Fourier usando una metodología de cálculo numérico aplicada a funciones radiales para el átomo de hidrógeno y el oscilador armónico en dos dimensiones además de átomos en tres dimensiones. En particular la transformada de Fourier en 2D de la parte radial de la función de onda es la transformada de Hankel y la transformada de Fourier en 3D de la parte radial de la función de onda corresponde a la transformada de Bessel esférica.

Para el caso de dos dimensiones se estudia la posibilidad de aplicar la metodología de Wong a transformadas de Hankel en donde el orden de dichas funciones tiene una dependencia respecto al número cuántico magnético $|\mathrm{m}|$. También se extiende su uso a funciones de Bessel de primer tipo de órdenes mayores a cero como $J_{1}(x), J_{2}(x)$ y $J_{3}(x)$.

Para el caso de tres dimensiones se desarrolla y aplica una nueva metodología en funciones de Bessel de orden semientero correspondientes a las funciones de Bessel esféricas $j_{0}(x), j_{1}(x), j_{2}(x), j_{3}(x)$ y $j_{4}(x)$. El orden de estas funciones tiene una dependencia respecto al número cuántico de momento angular $l$.

Se presentan las ventajas y dificultades que aparecen al utilizar este 
procedimiento para transformar funciones de tipo exponencial y Gaussiana con diferente comportamiento.

Los resultados numéricos obtenidos con esta metodología son comparados con otros procedimientos lo cual permite realizar un estudio completo de su alcance y factibilidad en dichas aplicaciones.

Las pruebas realizadas para sistemas cuánticos en $2 \mathrm{D}$ muestran que este método funciona para el átomo de hidrógeno dando resultados muy buenos en la región asintótica con órdenes de cuadratura bajos. Sin embargo para el oscilador armónico falla.

Las pruebas efectuadas en el caso 3D para funciones de base tipo Slater dan buenos resultados. En el caso de las funciones de base tipo Gaussianas se introdujo un parámetro para poder usar la metodología propuesta bajo ciertas restricciones.

Finalmente se probó el uso de esta metodología en la transformación de orbitales atómicos correspondientes a ciertos átomos para estudiar la dependencia con respecto a los valores de las constantes en el exponente de la exponencial de funciones de base tipo Slater. 


\section{Espacio de momentos}

El espacio de momentos y el espacio de posición son fundamentales en la química cuántica debido a su relación de reciprocidad originada por el principio de incertidumbre de Heisenberg y la no conmutatividad de los operadores. El estudio de ambos espacios permite una comprensión más precisa de los sistemas químicos. En esta parte es importante señalar que algunos fenómenos físicos se estudian con mayor facilidad a través del espacio de momentos.

\section{1 ¿Por qué estudiar el espacio de momentos?}

London [1] en sus estudios sobre la superconductividad establece que los electrones se mueven con la misma velocidad lo cual significa que se encuentran más correlacionados en el espacio de momentos por lo que aparece una condensación en este espacio. Dirac y Jordan [2] mostraron que la distribución de momentos es tan importante como la distribución espacial. Sin embargo, es más conocida y usada en química cuántica la densidad de carga $\rho(\boldsymbol{r})$.

Es fundamental, por ejemplo, encontrar expresiones teóricas para la distribución de momentos de los electrones en átomos ya que con estas se pueden determinar las curvas de densidad para el momento $p$ y calcular las formas de las líneas de Compton modificadas en átomos [3]. Es decir, el problema de determinar el perfil de la línea de Compton modificada se reduce a encontrar la función de densidad radial de momentos

$$
I(p)=\int|\widetilde{\Psi}(\boldsymbol{p})|^{2} p^{2} \mathrm{~d} \Omega .
$$

Además estas distribuciones son experimentalmente accesibles a través de la espectroscopia $(e, 2 e)[4]$.

Sabemos que la densidad de carga $\rho(\boldsymbol{r})$ en átomos y moléculas es un concepto muy importante el cual permite interpretar algunos fenómenos químicos. Por ejemplo los conceptos empíricos estrechamente relacionados con la densidad de carga son las longitudes de enlace, ángulos, radios atómicos, radios de Van der Waals, electronegatividad, etc [5]. También es posible obtener de manera experimental la densidad de carga $\rho(\boldsymbol{r})$ a través de la difracción Rayleigh de rayos X [6].

Es importante destacar que el análisis de la densidad radial de momentos (2.1.1) nos permite obtener información complementaria para estudiar átomos y moléculas. 
La definición de los momentos $\left\langle p^{k}\right\rangle$ a partir de la densidad radial de momentos es

$$
\left\langle p^{k}\right\rangle=\int_{0}^{\infty} p^{k} I(p) \mathrm{d} p
$$

esto hace posible obtener algunos valores relacionados con los momentos de $p$ que tienen interpretaciones físicas importantes. Así tenemos que el momento con $k=2$ es proporcional a la energía cinética electrónica no relativista. También $\langle p\rangle$ es proporcional a la energía de intercambio de Slater-Dirac (con un alto grado de precisión) y $\left\langle p^{3}\right\rangle$ es aproximadamente proporcional al valor inicial de la función de Patterson en cristalografía de rayos X [7]. Se puede obtener una forma cuasirelativista de la energía cinética de los electrones dada por

$$
E=\frac{\left\langle p^{2}\right\rangle}{2 m_{e}}-\frac{\left\langle p^{4}\right\rangle}{8 m_{e}^{3} c^{2}}
$$

donde $m_{e}$ es la masa del electrón, $c$ es la velocidad de la luz en el vacío, así el primer término es la energía cinética no relativista y el segundo término es la corrección de masa-velocidad de Breit-Pauli [8] que involucra a $\left\langle p^{4}\right\rangle$.

En la Fig. (2.1) tenemos las gráficas correspondientes a la densidad radial en el espacio de posición y momentos para el átomo de Neón $(\mathrm{Ne})$ las cuales proporcionan distinta información en cada espacio. Por ejemplo la gráfica de la densidad de carga promediada esféricamente $\bar{\rho}(r)$ muestra un comportamiento monótonamente decreciente. Mientras que la gráfica para la densidad de momentos promediada esféricamente $\bar{\Pi}(p)$ presenta un máximo y después decae más lentamente que $\bar{\rho}(r)$. También mostramos la gráfica correspondiente a la distribución de carga radial $D(r)$ en donde observamos claramente las capas $K$ y $L$ del átomo de Neón. Por otro lado en la gráfica de la densidad radial de momentos $I(p)$ vemos que sólo aparece la capa $L$ para el mismo átomo de Neón. Debido a lo anterior existe una motivación e interés por estudiar y desarrollar una química cuántica en el espacio de momentos [9]. Asimismo con esto podemos apreciar la importancia de estudiar ambos espacios para tener una comprensión más completa sobre los sistemas químicos.

La resolución de la ecuación de Schrödinger con el enfoque de la química cuántica en el espacio de posición es fundamental pero también se puede formular el problema en el espacio de momentos aunque esta vía no esta completamente desarrollada. Entonces el modo más usado para obtener funciones y densidades en el espacio de momentos es a través de la transformada de Fourier. Por eso el interés en desarrollar metodologías numéricas que permitan calcular estas transformadas. 

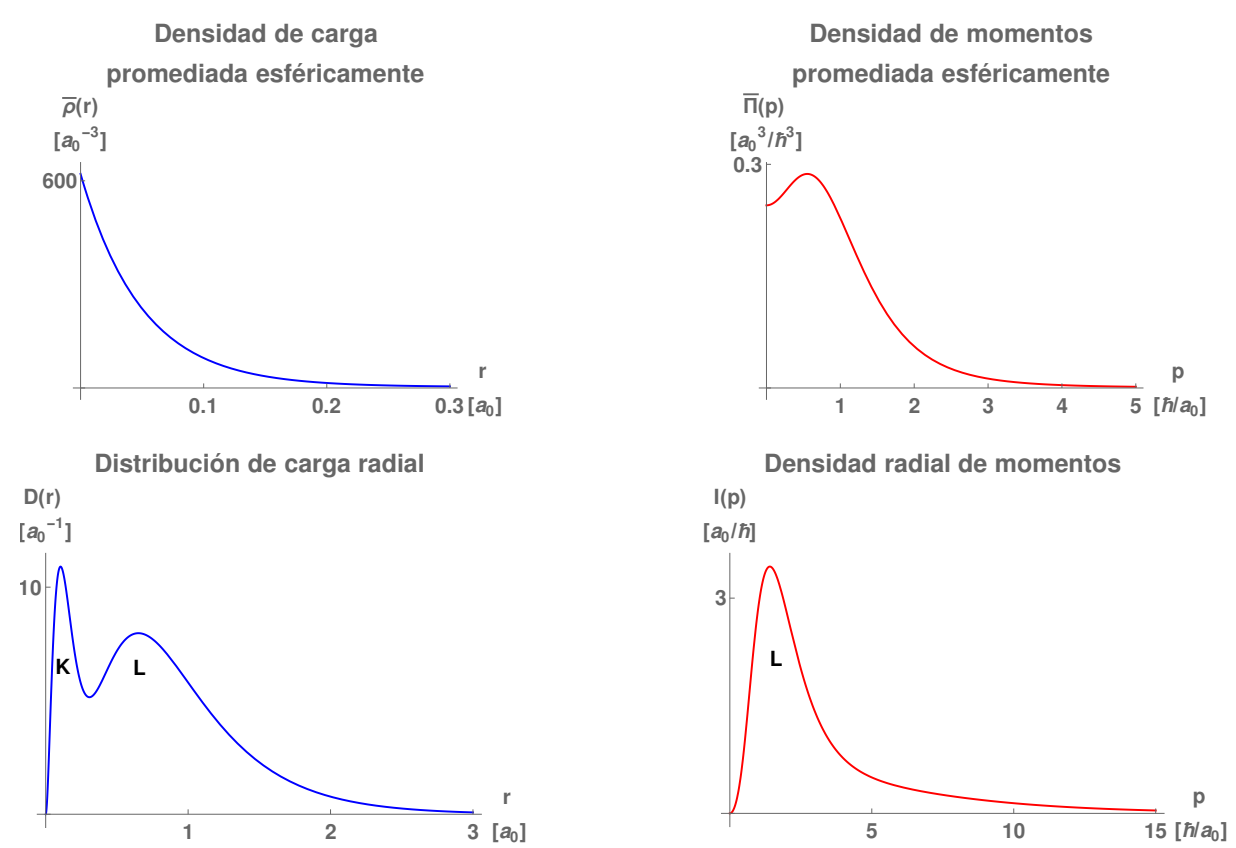

Figura 2.1: Representación en el espacio de posición y momentos de las densidades para el átomo de Neón $(N e)$.

\subsection{Representación en el espacio de momentos}

Veamos la forma en que se cambia de la representación en el espacio de posición $|\boldsymbol{r}\rangle$ a la representación en el espacio de momentos $|\boldsymbol{p}\rangle[10,11]$. Por la relación de cerradura se asume que cada ket $|\Psi\rangle$ tiene una expansión única sobre la base ortonormal continua $\left\{\left|u_{\alpha}\right\rangle\right\}$

$$
|\Psi\rangle=\int c_{\alpha}\left|u_{\alpha}\right\rangle \mathrm{d} \alpha
$$

siendo el coeficiente

$$
c_{\alpha}=\left\langle u_{\alpha} \mid \Psi\right\rangle \text {. }
$$

Para esto se establecen dos representaciones (bases ortonormales continuas) $\{|\boldsymbol{r}\rangle\}$ y $\{|\boldsymbol{p}\rangle\}$ dadas por

$$
\begin{array}{r}
|\boldsymbol{r}\rangle=\delta\left(\boldsymbol{r}-\boldsymbol{r}^{\prime}\right), \\
|\boldsymbol{p}\rangle=\left(\frac{1}{2 \pi \hbar}\right)^{-\frac{3}{2}} e^{\frac{\imath \boldsymbol{p} \cdot \boldsymbol{r}}{\hbar}} .
\end{array}
$$


donde los coeficientes $\langle\boldsymbol{r} \mid \Psi\rangle$ y $\langle\boldsymbol{p} \mid \Psi\rangle$ se calculan como

$$
\begin{array}{r}
\langle\boldsymbol{r} \mid \Psi\rangle=\int \delta\left(\boldsymbol{r}-\boldsymbol{r}^{\prime}\right) \Psi(\boldsymbol{r}) \mathrm{d} \boldsymbol{r}, \\
\langle\boldsymbol{p} \mid \Psi\rangle=\int\left(\frac{1}{2 \pi \hbar}\right)^{-\frac{3}{2}} e^{-\frac{\imath \boldsymbol{p} \cdot \boldsymbol{r}}{\hbar}} \Psi(\boldsymbol{r}) \mathrm{d} \boldsymbol{r} .
\end{array}
$$

El cambio de una base a otra está determinado por

$$
\langle\boldsymbol{r} \mid \boldsymbol{p}\rangle=\langle\boldsymbol{p} \mid \boldsymbol{r}\rangle^{*}=\left(\frac{1}{2 \pi \hbar}\right)^{-\frac{3}{2}} e^{\frac{\imath \boldsymbol{p} \cdot \boldsymbol{r}}{\hbar}} .
$$

Así que considerando un ket $|\Psi\rangle$ el cual queda representado como $\langle\boldsymbol{r} \mid \Psi\rangle=$ $\Psi(\boldsymbol{r})$ en el espacio de posición. Mientras que la representación en el espacio de momentos esta dada por $\langle\boldsymbol{p} \mid \Psi\rangle=\widetilde{\Psi}(\boldsymbol{p})$.

La fórmula para el cambio de representación al espacio de posición es

$$
\begin{gathered}
\langle\boldsymbol{r} \mid \Psi\rangle=\int\langle\boldsymbol{r} \mid \boldsymbol{p}\rangle\langle\boldsymbol{p} \mid \Psi\rangle \mathrm{d} \boldsymbol{p}, \\
\Psi(\boldsymbol{r})=\left(\frac{1}{2 \pi \hbar}\right)^{-\frac{3}{2}} \int e^{\frac{\imath \boldsymbol{p} \cdot \boldsymbol{r}}{\hbar}} \widetilde{\Psi}(\boldsymbol{p}) \mathrm{d} \boldsymbol{p} .
\end{gathered}
$$

En nuestro caso estamos interesados en el espacio de momentos

$$
\langle\boldsymbol{p} \mid \Psi\rangle=\int\langle\boldsymbol{p} \mid \boldsymbol{r}\rangle\langle\boldsymbol{r} \mid \Psi\rangle \mathrm{d} \boldsymbol{r}
$$

por lo que tenemos

$$
\widetilde{\Psi}(\boldsymbol{p})=\left(\frac{1}{2 \pi \hbar}\right)^{-\frac{3}{2}} \int e^{-\frac{\imath \boldsymbol{p} \cdot \boldsymbol{r}}{\hbar}} \Psi(\boldsymbol{r}) \mathrm{d} \boldsymbol{r},
$$

la ecuación anterior representa la transformada de Fourier de la función $\Psi(\boldsymbol{r})$. Por lo cual $\Psi(\boldsymbol{r})$ y $\widetilde{\Psi}(\boldsymbol{p})$ están relacionadas a través de la transformada de Fourier.

La interpretación física de esto es que cualquier función de onda $\Psi(\boldsymbol{r})$ puede expresarse como una superposición de estados $e^{\frac{i p \cdot r}{\hbar}}$ con momento definido $|\boldsymbol{p}\rangle$.

Además es importante mencionar que los operadores $|\boldsymbol{r}\rangle$ y $\quad|\boldsymbol{p}\rangle$ son equivalentes con el operador unitario dado explícitamente por la transformada de Fourier, esto significa que tienen el mismo espectro. 
Por otro lado, se puede pensar en reformular la ecuación de Schrödinger en el espacio de momentos para obtener sus soluciones en términos de $|\boldsymbol{p}\rangle[10]$. Pero este tratamiento no es muy común por lo que no se ha desarrollado de forma extendida su aplicación en la química cuántica.

El estudio de la función de distribución de momentos $\widetilde{\Psi}(\boldsymbol{p})$ que vamos a tratar se basa en las transformadas de Fourier de las funciones de onda espaciales $\Psi(\boldsymbol{r})$. Por lo que a partir de la teoría de Dirac [12] tenemos que $\widetilde{\Psi}(\boldsymbol{p})$ es simplemente la transformada de Fourier en tres dimensiones (3.3.1) de la función de onda $\Psi(\boldsymbol{r})$.

Si consideramos sistemas atómicos o moleculares con $n$ electrones la función de onda de momentos $\widetilde{\Psi}\left(\boldsymbol{p}_{1}, \boldsymbol{p}_{2}, \ldots, \boldsymbol{p}_{n}\right)$ se obtiene de la función de onda espacial $\Psi\left(\boldsymbol{r}_{1}, \boldsymbol{r}_{2}, \ldots, \boldsymbol{r}_{n}\right)$ usando la transformada de Fourier de $3 n$ dimensiones [3]

$$
\begin{aligned}
\widetilde{\Psi}\left(\boldsymbol{p}_{1}, \boldsymbol{p}_{2}, \ldots, \boldsymbol{p}_{n}\right)=\left(\frac{1}{2 \pi}\right)^{\frac{3 n}{2}} \int_{-\infty}^{\infty} \ldots \int_{-\infty}^{\infty} \Psi\left(\boldsymbol{r}_{1}, \boldsymbol{r}_{2}, \ldots, \boldsymbol{r}_{n}\right) \\
\\
\times e^{-\imath\left(\boldsymbol{p}_{1} \cdot \boldsymbol{r}_{1}+\cdots+\boldsymbol{p}_{n} \cdot \boldsymbol{r}_{n}\right)} \mathrm{d} \boldsymbol{r}_{1} \mathrm{~d} \boldsymbol{r}_{2} \cdots \mathrm{d} \boldsymbol{r}_{n} .
\end{aligned}
$$




\section{Transformada de Fourier}

Las transformadas integrales aparecen en múltiples problemas aplicados [13] en donde se requiere pasar de un espacio de funciones a otro a través del kernel integral $\mathcal{K}(p, x)$ mediante el operador

$$
\widetilde{f}(p)=\int_{-\infty}^{\infty} f(x) \mathcal{K}(p, x) \mathrm{d} x
$$

así tenemos la proyección de todo el espacio de funciones $f(x) \in U$ sobre $\mathcal{K}(p, x)$ en cada valor de $\widetilde{f}(p) \in V$, donde $U$ y $V \in L^{1}\left(\mathbb{R}^{n}\right)$ son funciones de tipo Lebesgue integrable.
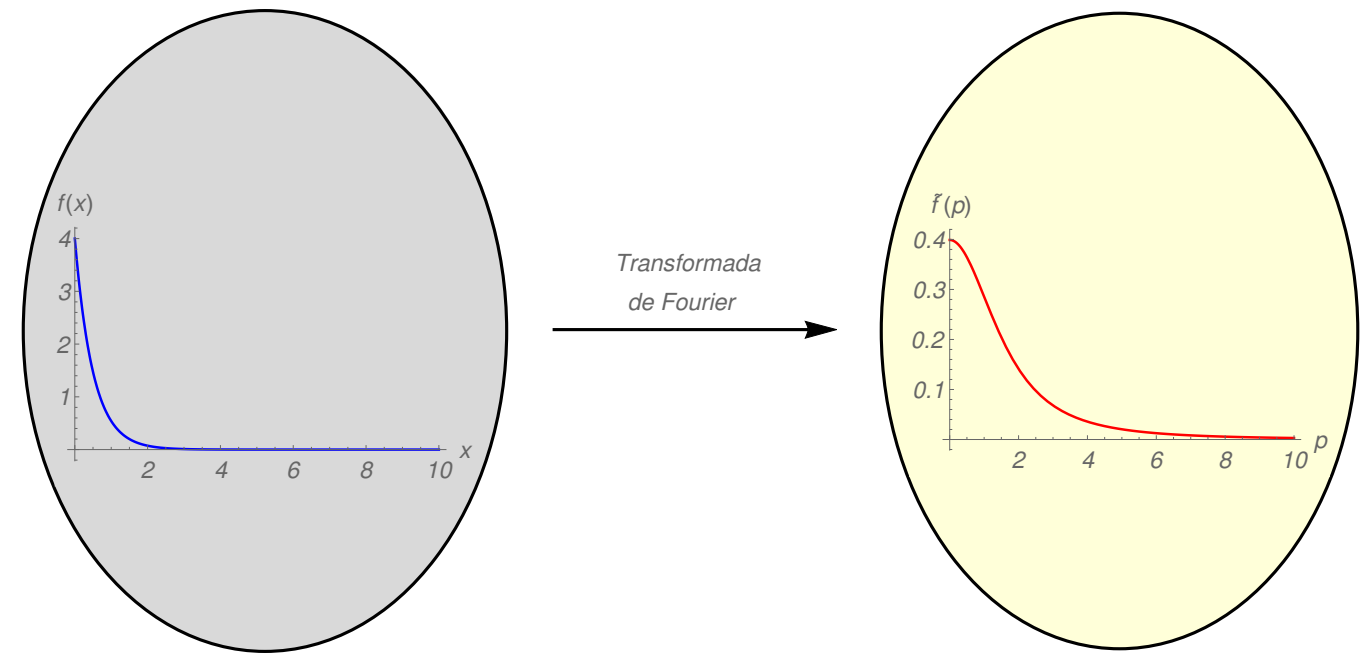

Figura 3.1: Esquema de la transformada de Fourier de $f(x)=e^{-x}$.

Existen varias transformadas integrales para distintos fines y dependiendo del tipo de kernel $\mathcal{K}(p, x)$ en el operador reciben su nombre [14].

En particular en este trabajo estamos interesados en usar la transformada de Fourier en dos y tres dimensiones. Así tenemos que el kernel integral usado en (3.0.13) se expresa en términos de la exponencial compleja $\mathcal{K}(p, x)=e^{-\imath p x}$.

Estamos enfocados en el estudio de las transformadas de Fourier en espacios Euclidianos debido a la interpretación dada a las variables ( $x$-posición, $p$-momento) y a la dimensionalidad del problema. Además en nuestro caso por la existencia de variables conjugadas o complementarias $(x, p)$ que se relacionan con el principio de incertidumbre de Heisenberg usamos la transformada de Fourier para pasar de una representación a la otra. 


\subsection{Transformada de Fourier en una dimensión}

La definición de la transformada de Fourier $\widetilde{f}(p)$ de $f(x)$ en una dimensión está dada por

$$
\tilde{f}(p)=\frac{1}{\sqrt{2 \pi}} \int_{-\infty}^{\infty} f(x) e^{-\imath p x} \mathrm{~d} x
$$

y la transformada inversa de Fourier es

$$
f(x)=\frac{1}{\sqrt{2 \pi}} \int_{-\infty}^{\infty} \widetilde{f}(p) e^{\imath p x} \mathrm{~d} p .
$$

Se tiene que $f(x)$ debe satisfacer las condiciones de Dirichlet en su dominio de integración y la integral $\int_{-\infty}^{\infty}|f(x)| \mathrm{d} x$ debe ser convergente [3].

Se ha trabajado ampliamente en usar la transformada de Fourier unidimensional para transformar la representación de la función de onda dependiente en una variable (posición o momento) veáse [15].

\subsection{Transformada de Fourier en dos dimensiones}

Nuestro trabajo comienza con el estudio de la transformada de Fourier en dos dimensiones. Así consideramos la transformada de Fourier $\widetilde{f}(\boldsymbol{p})=\widetilde{f}\left(p_{1}, p_{2}\right)$ de $f(\boldsymbol{x})=f\left(x_{1}, x_{2}\right)$ la cual está dada por

$$
\widetilde{f}\left(p_{1}, p_{2}\right)=\frac{1}{2 \pi} \int_{-\infty}^{\infty} \int_{-\infty}^{\infty} f\left(x_{1}, x_{2}\right) e^{-\imath\left(p_{1} x_{1}+p_{2} x_{2}\right)} \mathrm{d} x_{1} \mathrm{~d} x_{2},
$$

y su transformada inversa de Fourier es

$$
f\left(x_{1}, x_{2}\right)=\frac{1}{2 \pi} \int_{-\infty}^{\infty} \int_{-\infty}^{\infty} \tilde{f}\left(p_{1}, p_{2}\right) e^{\imath\left(p_{1} x_{1}+p_{2} x_{2}\right)} \mathrm{d} p_{1} \mathrm{~d} p_{2} .
$$

Donde sabemos que en un espacio de dos dimensiones el producto punto entre dos vectores se define como $\boldsymbol{p} \cdot \boldsymbol{x}=p_{1} x_{1}+p_{2} x_{2}$. El cual aparece en el kernel integral $\mathcal{K}(\boldsymbol{p}, \boldsymbol{x})=e^{ \pm \imath \boldsymbol{p} \cdot \boldsymbol{x}}$.

Existe una relación entre la transformada de Fourier en dos dimensiones y la transformada de Hankel al cambiar el sistema de coordenadas.

\subsubsection{Transformada de Hankel}

En este caso al transformar el sistema de coordenadas cartesianas a coordenadas polares los cambios de variable correspondientes son

$$
x_{1}=r \cos \theta, \quad x_{2}=r \operatorname{sen} \theta, \quad p_{1}=p \cos \phi, \quad p_{2}=p \operatorname{sen} \phi,
$$




$$
r=\sqrt{x_{1}^{2}+x_{2}^{2}}, \quad p=\sqrt{p_{1}^{2}+p_{2}^{2}},
$$

de donde el elemento de volumen y el producto punto se representan como

$$
\mathrm{d} x_{1} \mathrm{~d} x_{2}=r \mathrm{~d} r \mathrm{~d} \theta, \quad p_{1} x_{1}+p_{2} x_{2}=p r \cos (\theta-\phi) .
$$

Considerando una función de tipo separable $f(\boldsymbol{r})=f(r, \phi)$ tal que tenemos el producto de la parte radial y angular como $f(\boldsymbol{r})=f(r) e^{\imath m \phi}$ que al sustituirse en (3.2.1)

$$
\begin{gathered}
\tilde{f}(\boldsymbol{p})=\frac{1}{2 \pi} \int_{0}^{\infty} f(\boldsymbol{r}) e^{-\imath \boldsymbol{p} \cdot \boldsymbol{r}} \mathrm{d} \boldsymbol{r} \\
\tilde{f}(\boldsymbol{p})=\frac{1}{2 \pi} \int_{0}^{\infty} \int_{0}^{2 \pi} f(r) e^{\imath m \phi} e^{-\imath p r \cos \left(\phi-\phi_{p}\right)} r \mathrm{~d} r \mathrm{~d} \phi
\end{gathered}
$$

donde $\phi_{p}$ es el ángulo entre el vector resultante y el eje $\phi=0$. Definiendo entonces $\theta=\phi-\phi_{p}$ y utilizando la periodicidad del integrando sobre las variables angulares $[3,13,16]$

$$
\widetilde{f}(\boldsymbol{p})=\imath^{m} e^{\imath m \phi_{p}} \int_{0}^{\infty} f(r) r \mathrm{~d} r\left[\int_{0}^{2 \pi} e^{\imath m \theta} e^{\imath p r \cos \theta} \mathrm{d} \theta\right],
$$

el paréntesis es la representación integral de la función de Bessel de $m$-ésimo orden con argumento $p r$ multiplicado por $2 \pi \imath^{m}$

$$
\widetilde{f}(\boldsymbol{p})=\imath^{m} e^{\imath m \phi_{p}} \int_{0}^{\infty} f(r) J_{m}(p r) r \mathrm{~d} r
$$

por lo que la integral $\int_{0}^{\infty} f(r) J_{m}(p r) r \mathrm{~d} r$ se conoce como la transformada de Hankel de $m$-ésimo orden de la función radial $f(r)$. Así la transformada de Fourier en 2D está relacionada con la transformada de Hankel de la función radial.

\subsubsection{Simetría circular}

Es importante señalar que los sistemas en dos dimensiones muestran con frecuencia simetría circular. Si existe simetría circular se tiene que

$$
\begin{aligned}
f\left(x_{1}, x_{2}\right)=f(\boldsymbol{r}), & r^{2}=x_{1}^{2}+x_{2}^{2}, \\
\widetilde{f}\left(p_{1}, p_{2}\right)=\widetilde{f}(\boldsymbol{p}), & p^{2}=p_{1}^{2}+p_{2}^{2},
\end{aligned}
$$


y además

$$
x_{1}+\imath x_{2}=r e^{\imath \phi}, \quad p_{1}+\imath p_{2}=p e^{\imath \phi_{p}} .
$$

$\mathrm{Al}$ aplicar la transformada de Fourier en dos dimensiones a una función se prefiere pasar a coordenadas polares e integrar sobre la variable angular [13], tal y como se hizo en (3.2.4). También si la función $f(\boldsymbol{r})$ resulta circularmente simétrica $(m=0)$ consideramos que el ángulo $\phi$ está entre el vector $\boldsymbol{r}$ y el eje $\phi=0$. Con lo que obtenemos la transformada de Hankel de orden cero de la función $f(\boldsymbol{r})$

$$
\widetilde{f}(\boldsymbol{p})=\int_{0}^{\infty} r f(r) J_{0}(p r) \mathrm{d} r
$$

mientras que la transformada inversa de Hankel de orden cero es

$$
f(\boldsymbol{r})=\int_{0}^{\infty} p \widetilde{f}(p) J_{0}(p r) \mathrm{d} p
$$

\subsection{Transformada de Fourier en tres dimensiones}

Sea la transformada de Fourier en tres dimensiones

$$
\widetilde{f}(\boldsymbol{p})=\left(\frac{1}{2 \pi}\right)^{\frac{3}{2}} \int_{-\infty}^{\infty} \int_{-\infty}^{\infty} \int_{-\infty}^{\infty} f(\boldsymbol{r}) e^{-\imath(\boldsymbol{p} \cdot \boldsymbol{r})} \mathrm{d} \boldsymbol{r}
$$

si usamos coordenadas esféricas tenemos

$$
\widetilde{f}\left(p, \theta_{p}, \phi_{p}\right)=\left(\frac{1}{2 \pi}\right)^{\frac{3}{2}} \int_{0}^{\infty} \int_{0}^{\pi} \int_{0}^{2 \pi} f(r, \theta, \phi) e^{-\imath \mu} r^{2} \operatorname{sen} \theta \mathrm{d} r \mathrm{~d} \theta \mathrm{d} \phi,
$$

donde los cambios de variable correspondientes son

$\boldsymbol{p}=p\left(\operatorname{sen} \theta_{p} \cos \phi_{p}, \operatorname{sen} \theta_{p} \operatorname{sen} \phi_{p}, \cos \theta_{p}\right), \quad \boldsymbol{r}=r(\operatorname{sen} \theta \cos \phi, \operatorname{sen} \theta \operatorname{sen} \phi, \cos \theta)$,

$$
\mu=\operatorname{pr}\left(\operatorname{sen} \theta \operatorname{sen} \theta_{p} \cos \left(\phi_{p}-\phi\right)+\cos \theta \cos \theta_{p}\right)
$$

La integral (3.3.2) se usa para transformar la función de onda del átomo hidrogenoide en el espacio de posición al espacio de momentos. Así en el artículo de Podolsky y Pauling [17] se efectúa el tratamiento y desarrollo de esta integral para obtener la expresión general de las funciones hidrogenoides en el espacio de momentos. 


\subsubsection{Transformada de Bessel esférica}

En este caso utilizamos el sistema de coordenadas esféricas $f(\boldsymbol{r})=f(r, \theta, \phi)$. A partir de la ecuación (3.3.1) con una función $f(\boldsymbol{r})=f(r) Y_{l, m}(\theta, \phi)$ separable la transformada integral se convierte en

$$
\tilde{f}(\boldsymbol{p})=\left(\frac{1}{2 \pi}\right)^{\frac{3}{2}} \int_{0}^{\infty} \int_{0}^{\pi} \int_{0}^{2 \pi} f(r) Y_{l, m}(\theta, \phi) e^{-\imath p r \omega} r^{2} \operatorname{sen} \theta \mathrm{d} r \mathrm{~d} \theta \mathrm{d} \phi,
$$

donde se define $\omega=\left(\operatorname{sen} \theta \operatorname{sen} \theta_{p} \cos \left(\phi_{p}-\phi\right)+\cos \theta \cos \theta_{p}\right)$ y también $Y_{l, m}(\theta, \phi)=N e^{-\imath m \phi} P_{l}^{m}(\cos \theta)$ con su correspondiente constante de normalización $N$

$$
\tilde{f}(\boldsymbol{p})=\left(\frac{N}{2 \pi}\right)^{\frac{3}{2}} \int_{0}^{\infty} f(r) r^{2} \mathrm{~d} r\left[\int_{0}^{\pi} \int_{0}^{2 \pi} e^{-\imath m \phi} P_{l}^{m}(\cos \theta) e^{-\imath p r \omega} \operatorname{sen} \theta \mathrm{d} \theta \mathrm{d} \phi\right],
$$

considerando el tratamiento en $[16,17]$ las integrales en el corchete llevan a

$$
\widetilde{f}(\boldsymbol{p})=(-\imath)^{l}\left(\frac{2}{\pi}\right)^{\frac{1}{2}} Y_{l, m}\left(\theta_{p}, \phi_{p}\right) \int_{0}^{\infty} r^{2} f(r) j_{l}(p r) \mathrm{d} r
$$

la integral $\int_{0}^{\infty} r^{2} f(r) j_{l}(p r) \mathrm{d} r$ corresponde a la transformada de Bessel esférica. Por lo que la transformada de Fourier en 3D está relacionada con la transformada de Bessel esférica de la función radial.

En general se considera que la transformada de Fourier de una función radial es también una función radial para dimensiones de $n \geq 2$ [16], donde si $n=2$ tenemos a la transformada de Hankel relacionada con funciones de Bessel de orden entero. Para $n=3$ lo que aparece en el integrando de las transformadas de funciones radiales es una función de Bessel esférica o de orden semientero.

\subsection{Transformada de Fourier en $n$-dimensiones}

Existe una generalización en dimensiones mayores donde la función de varias variables $f\left(x_{1}, x_{2}, \ldots, x_{n}\right)=f(\boldsymbol{r})$ y $r=\sqrt{x_{1}^{2}+x_{2}^{2}+\ldots+x_{n}^{2}}$. Considerando la transformada de Fourier como

$$
\begin{aligned}
\tilde{f}\left(p_{1}, p_{2}, \ldots, p_{n}\right)=\left(\frac{1}{2 \pi}\right)^{\frac{n}{2}} \int_{-\infty}^{\infty} & \ldots \int_{-\infty}^{\infty} f\left(x_{1}, x_{2}, \ldots, x_{n}\right) \\
\times & e^{-\imath\left(p_{1} x_{1}+p_{2} x_{2}+\ldots+p_{n} x_{n}\right)} \mathrm{d} x_{1} \mathrm{~d} x_{2} \ldots \mathrm{d} x_{n},
\end{aligned}
$$


donde $\boldsymbol{p} \cdot \boldsymbol{r}=p_{1} x_{1}+p_{2} x_{2}+\ldots+p_{n} x_{n}$ es el producto interno correspondiente.

En términos generales si se conoce la función de onda espacial $\Psi\left(r_{1}, r_{2}, \ldots, r_{n}\right)$ entonces determinar la función de onda de momentos $\widetilde{\Psi}\left(p_{1}, p_{2}, \ldots, p_{n}\right)$ consiste en aplicar la transformada de Fourier a la función $\Psi\left(r_{1}, r_{2}, \ldots, r_{n}\right)$.

\subsection{Transformada de Dirac-Fourier de funciones de onda en el espacio de posición}

La transformada de Dirac-Fourier se define como en (2.2.12)

$$
\widetilde{\Psi}(\boldsymbol{P}, \boldsymbol{\sigma})=\left(\frac{1}{2 \pi}\right)^{\frac{3 n}{2}} \int_{-\infty}^{\infty} \Psi(\boldsymbol{R}, \boldsymbol{\sigma}) e^{-\imath(\boldsymbol{P} \cdot \boldsymbol{R})} \mathrm{d} \boldsymbol{R},
$$

donde $\boldsymbol{P}=\left(\boldsymbol{p}_{1}, \boldsymbol{p}_{2}, \ldots, \boldsymbol{p}_{n}\right)$ son las coordenadas de momento, $\boldsymbol{R}=$ $\left(\boldsymbol{r}_{1}, \boldsymbol{r}_{2}, \ldots, \boldsymbol{r}_{n}\right)$ son las coordenadas de posición, $\boldsymbol{\sigma}=\left(\sigma_{1}, \sigma_{2}, \ldots, \sigma_{n}\right)$ son las coordenadas de espín y $\mathrm{d} \boldsymbol{R}=\mathrm{d} \boldsymbol{r}_{1} \mathrm{~d} \boldsymbol{r}_{2} \cdots \mathrm{d} \boldsymbol{r}_{n}$ es el elemento de volumen [18].

De acuerdo a [19-21] se considera que las variables de espín no aparecen en la transformación de Dirac-Fourier. Por lo que se puede expresar en términos de los orbitales naturales $\boldsymbol{\chi}(\boldsymbol{r})=\left\{\chi_{1}(\boldsymbol{r}), \chi_{2}(\boldsymbol{r}), \ldots, \chi_{n}(\boldsymbol{r})\right\}$ los cuales son combinaciones lineales de un conjunto de funciones de base linealmente independientes $\boldsymbol{\phi}(\boldsymbol{r})=\left\{\phi_{1}(\boldsymbol{r}), \phi_{2}(\boldsymbol{r}), \ldots, \phi_{n}(\boldsymbol{r})\right\}$ como en la aplicación para orbitales atómicos (7.3). Así se definen las transformadas de Fourier $\widetilde{\chi}(\boldsymbol{p})$ y $\widetilde{\phi}(\boldsymbol{p})$ de los orbitales naturales $\boldsymbol{\chi}(\boldsymbol{r})$ y las funciones de base $\boldsymbol{\phi}(\boldsymbol{r})$ como $(3.5 .1)$

$$
\begin{aligned}
\tilde{\chi}_{i}(\boldsymbol{p}) & =\left(\frac{1}{2 \pi}\right)^{\frac{3 n}{2}} \int_{-\infty}^{\infty} \chi_{i}(\boldsymbol{r}) e^{-\imath(\boldsymbol{p} \cdot \boldsymbol{r})} \mathrm{d} \boldsymbol{r} \\
& =\left(\frac{1}{2 \pi}\right)^{\frac{3 n}{2}} e^{-\imath\left(\boldsymbol{p} \cdot \boldsymbol{A}_{i}\right)} \int_{-\infty}^{\infty} \chi_{i}\left(\boldsymbol{r}_{i}\right) e^{-\imath\left(\boldsymbol{p} \cdot \boldsymbol{r}_{i}\right)} \mathrm{d} \boldsymbol{r}_{i},
\end{aligned}
$$

$\mathrm{y}$

$$
\begin{aligned}
\widetilde{\phi}_{i}(\boldsymbol{p}) & =\left(\frac{1}{2 \pi}\right)^{\frac{3 n}{2}} \int_{-\infty}^{\infty} \phi_{i}(\boldsymbol{r}) e^{-\imath(\boldsymbol{p} \cdot \boldsymbol{r})} \mathrm{d} \boldsymbol{r} \\
& =\left(\frac{1}{2 \pi}\right)^{\frac{3 n}{2}} e^{-\imath\left(\boldsymbol{p} \cdot \boldsymbol{A}_{i}\right)} \int_{-\infty}^{\infty} \phi_{i}\left(\boldsymbol{r}_{i}\right) e^{-\imath\left(\boldsymbol{p} \cdot \boldsymbol{r}_{i}\right)} \mathrm{d} \boldsymbol{r}_{i}
\end{aligned}
$$

donde $\boldsymbol{r}=\boldsymbol{A}_{i}+\boldsymbol{r}_{i}$ tal que $\boldsymbol{A}_{i}$ es la posición del centro para $\phi_{i}$ a partir de la cual se define $\boldsymbol{r}_{i}$ [18]. 


\subsection{Transformada de Dirac-Fourier de orbitales atómicos}

La ecuación de Schrödinger para una partícula con un potencial central en coordenadas polares esféricas resulta separable. Además sabemos que las soluciones en el espacio de posición se escriben como

$$
\phi_{n, l, m}(\boldsymbol{r})=f_{n, l}(r) Y_{l, m}(\theta, \phi),
$$

donde $n, l$ y $m$ son los números cuánticos y $Y_{l, m}(\theta, \phi)$ corresponde a la función armónica esférica normalizada para $m \geq 0$ [22].

Para poder transformar $\phi(\boldsymbol{r})$ se deben cambiar las variables a su centro $\boldsymbol{A}$ y utilizar la expansión de Messiah en (3.5.3) tal que

$$
e^{-\imath(\boldsymbol{p} \cdot \boldsymbol{r})}=4 \pi \sum_{l=0}^{\infty} \sum_{m=-l}^{l}(-\imath)^{l} j_{l}(p r) Y_{l, m}^{*}(\theta, \phi) Y_{l, m}\left(\theta_{p}, \phi_{p}\right),
$$

así $j_{l}(p r)$ es una función de Bessel esférica de orden $l$ mientras que $\theta_{p}$ y $\phi_{p}$ son las variables angulares en el espacio de momentos. Al integrar sobre la parte angular en la transformada de Dirac-Fourier y usando propiedades de ortogonalidad de los armónicos esféricos tenemos que

$$
\widetilde{\phi}_{n, l, m}(\boldsymbol{p})=e^{-\imath(\boldsymbol{p} \cdot \boldsymbol{A})}(-\imath)^{l} Y_{l, m}\left(\theta_{p}, \phi_{p}\right)\left(\frac{2}{\pi}\right)^{\frac{1}{2}} \int_{0}^{\infty} r^{2} f_{n, l}(r) j_{l}(p r) \mathrm{d} r,
$$

se debe mencionar que esto es una manera análoga a las expresiones dadas en (3.3.4) y (3.3.5).

El factor de fase $e^{-\imath(\boldsymbol{p} \cdot \boldsymbol{A})}$ se descarta en sistemas atómicos seleccionando como origen el núcleo. Por lo tanto tenemos que

$$
\widetilde{\phi}_{n, l, m}(\boldsymbol{p})=u_{n, l}(p) Y_{l, m}\left(\theta_{p}, \phi_{p}\right),
$$

identificando a $u_{n, l}(p)$ como la parte radial del orbital en el espacio de momentos

$$
\begin{aligned}
u_{n, l}(p) & =(-\imath)^{l}\left(\frac{2}{\pi}\right)^{\frac{1}{2}} \int_{0}^{\infty} r^{2} f_{n, l}(r) j_{l}(p r) \mathrm{d} r \\
& =\frac{(-\imath)^{l}}{p} h_{n, l}(p)
\end{aligned}
$$

donde $h_{n, l}(p)$ es la transformada de Hankel de orden $\nu=l+\frac{1}{2}$ ver (3.2.8) de la función $r f_{n, l}(r)$ definida como

$$
h_{n, l}(p)=\int_{0}^{\infty}\left[r f_{n, l}(r)\right] J_{l+\frac{1}{2}}(p r)(p r)^{\frac{1}{2}} \mathrm{~d} r .
$$

Es importante mencionar que [18] 
- $u_{n, l}(p)$ es una transformación que depende en la simetría de la parte radial de $\phi_{n, l, m}(\boldsymbol{r})$ la cual se relaciona con una función de Bessel esférica del mismo orden que el número cuántico orbital $l$.

- Como $u_{n, l}(p)$ está determinada por esta transformación dependiente de la simetría y no por la transformada de Fourier unidimensional. Se debe tener cuidado en hacer suposiciones acerca de $u_{n, l}(p)$ a partir de $f_{n, l}(r)$ en particular cuando se comparan orbitales con diferente $l$. 


\section{Aproximaciones numéricas}

El problema matemático general consiste en resolver integrales de la forma

$$
I(\eta, \nu, p)=\int_{0}^{\infty} x^{\eta} f(x) J_{\nu}(p x) \mathrm{d} x
$$

donde la integración analítica es imposible en muchos casos [14]. Así por ejemplo en algunas aplicaciones relacionadas con fenómenos ondulatorios es necesario calcular la integral numéricamente.

Las integrales referentes a la ecuación (4.0.7) son difíciles de resolver numéricamente debido a la oscilación en el integrando y además esto se intensifica cuando el valor del argumento $p$ crece [14, 23-34].

Los métodos numéricos para resolver integrales con funciones de Bessel se restringen a los casos donde $f(x)$ es una función suave.

Un método para resolver este tipo de integrales consiste en transformar la integral (4.0.7) en una integral doble [35] lo cual se abordará en la sección (6.5).

También existe un método para calcular integrales que implican funciones de Bessel de primer tipo $J_{0}(x)$. Esto mediante cuadraturas Gaussianas no estándar basadas en funciones de peso que se representan a través de funciones de Bessel de tercer tipo $K_{0}(x)$ [36]. Este método es el que se estudia y se aplica para resolver el problema de transformar al espacio de momentos funciones radiales del átomo de hidrógeno y el oscilador armónico en 2D. Además se examina su extensión y uso en funciones de base tipo Slater, Gaussianas y orbitales atómicos en 3D.

Es importante mencionar que en el trabajo original de Wong [36] sólo se estudian funciones de Bessel de orden entero y de tipo $J_{0}(x)$ aplicadas a funciones que no presentan comportamiento exponencial ni Gaussiano. Además este es un excelente método para regiones asintóticas, donde existe dificultad en la aplicación de métodos tradicionales debido a la oscilación de las funciones de peso en el integrando (4.0.7).

Sin embargo en este trabajo estudiamos la región no asintótica y su aplicabilidad a órdenes más altos de $J_{\nu}(x)$, donde el valor de $\nu$ tiene relación con el número cuántico magnético $|m|$ en los sistemas cuánticos en 2D y su extensión a sistemas en 3D.

Las relaciones entre las funciones de Bessel de distinto orden y tipo pueden encontrarse en diversos tratados de funciones especiales [37-40]. 


\subsection{Cuadratura Gaussiana}

En muchas aplicaciones es imprescindible calcular numéricamente integrales lo cual consiste en obtener el valor aproximado de una integral definida

$$
\int_{a}^{b} f(x) \mathrm{d} x
$$

Existen diversos esquemas para resolver (4.1.1) dependiendo del comportamiento de la función $f(x)$ a integrar y del intervalo de integración $[a, b]$.

En este trabajo estamos interesados en integrales que tienen un intervalo de integración que se extiende a infinito $[0, \infty]$ y la función $f(x)$ oscila rápidamente. Para superar las dificultades mencionadas se puede usar el método para integrar un producto de funciones mediante cuadraturas con peso, donde se incorpora el comportamiento problemático en la función de peso $w(x)$ convirtiéndose en un factor en el integrando mientras que el otro factor $f(x)$ representa una función bien comportada [41,42].

Por lo que la cuadratura con peso se representa mediante una fórmula de integración numérica como

$$
\int_{a}^{b} f(x) w(x) \mathrm{d} x=\sum_{k=1}^{N} w_{k} f\left(x_{k}\right)+E_{N}(f),
$$

donde se cumple que la función de peso $w(x) \geq 0$ es definida positiva en el intervalo $[a, b]$. Además de que todos sus momentos $\mu_{n}=\int_{a}^{b} x^{n} w(x) \mathrm{d} x$ con $n=0,1,2, \ldots$, existen y son finitos $[42,43]$. Es importante mencionar que en nuestro caso $J_{\nu}(x)$ no cumple con la primera condición sobre la positividad de la función de peso $w(x)$. Asimismo no es una función bien comportada por su fuerte oscilación en la región asintótica.

Así tenemos que la fórmula de cuadratura (4.1.2) es una fórmula de Newton-Cotes

$$
\int_{a}^{b} f(x) w(x) \mathrm{d} x \approx \sum_{k=1}^{N} w_{k} f\left(x_{k}\right)
$$

que consiste en aproximar el lado izquierdo de la ecuación (4.1.2) a través de una suma finita que involucra $n$ valores de $f\left(x_{k}\right)$ evaluados en distintos nodos $x_{k}$ seleccionados adecuadamente.

Se considera que la fórmula (4.1.3) es de tipo interpolatorio si y sólo si es exacta para la base de polinomios $\left\{\pi_{j}\right\}_{j=0}^{n}=\left\{1, x, \ldots, x^{n-1}\right\}$, donde el problema de encontrar los pesos $w_{k}$ consiste en resolver el sistema lineal 
(matriz de Vandermonde transpuesta) que se obtiene imponiendo las $n$ condiciones de exactitud.

La fórmula de cuadratura tiene grado de precisión $d$ si es exacta para todo polinomio $p(x)$ de grado menor o igual a $d$. Una fórmula de tipo interpolatorio con $n$ nodos posee al menos grado de precisión $n-1$.

\subsection{Polinomios ortogonales}

Una sucesión de polinomios ortogonales $\left\{\pi_{n}\right\}_{n=0}^{\infty}$ forma una base por lo que $f, g \in \pi_{n}(x)$ definen el producto escalar con respecto a una función de peso $w(x)$ como

$$
\begin{gathered}
(f, g)=\int_{a}^{b} f(x) g(x) w(x) \mathrm{d} x, \\
\left(\pi_{j}, \pi_{k}\right)=0, \quad j \neq k .
\end{gathered}
$$

Lo anterior significa que dada una sucesión de polinomios ortogonales respecto a una función de peso cada $\pi_{n}(x)$ está determinado de forma única salvo por la multiplicación de una constante no nula. Por lo tanto todos los polinomios tienen el coeficiente con la potencia $x$ de mayor grado igual a 1 .

Otro aspecto fundamental de la teoría de polinomios ortogonales es la existencia de relaciones de recurrencia, las cuales son importantes para el cálculo efectivo de los polinomios ortogonales y de las fórmulas de cuadratura Gaussiana.

\subsection{Fórmulas de cuadratura Gaussiana y polinomios ortogonales}

La teoría sobre la construcción de fórmulas de cuadratura Gaussiana se basa en la teoría de polinomios ortogonales [44].

Existe una relación con las fórmulas interpolatorias en las cuales la aproximación se obtiene integrando el polinomio de interpolación $p(x)$ de la función $f(x)$ tal que a partir de (4.1.3) tenemos

$$
\begin{gathered}
\int_{a}^{b} f(x) w(x) \mathrm{d} x \approx \int_{a}^{b} p(x) w(x) \mathrm{d} x \\
\int_{a}^{b} p(x) w(x) \mathrm{d} x=\sum_{k=1}^{N} w_{k} f\left(x_{k}\right),
\end{gathered}
$$


donde suponemos que $p(x)$ es el polinomio interpolante de Lagrange de $f(x)$ en los puntos $x_{1}, \ldots, x_{n}$.

A partir de la definición de los polinomios interpolantes de Lagrange $\ell_{k}(x)=\prod_{\substack{l=1 \\ l \neq k}}^{n} \frac{x-x_{l}}{x_{k}-x_{l}}$ tenemos que en la base de Lagrange $\left\{\ell_{k}(x)\right\}_{1}^{n}$ se puede expresar $p(x)$ como

$$
p(x)=\sum_{k=1}^{N} f\left(x_{k}\right) \ell_{k}(x) .
$$

Así que la fórmula es de tipo interpolatorio si los pesos $w_{k}$ vienen dados por

$$
w_{k}=\int_{a}^{b} \ell_{k}(x) w(x) \mathrm{d} x, \quad k=1,2, \ldots, n .
$$

Cabe mencionar que la formulación en términos de las integrales de los polinomios de Lagrange es equivalente a encontrar la solución de la matriz de Vandermonde transpuesta.

Ahora definimos al polinomio ortogonal de $n$-ésimo grado relacionado con la función de peso $w(x)$ como $\pi_{n}(x)=\prod_{k=1}^{n}\left(x-x_{k}\right)$, el cual es ortogonal al conjunto de polinomios $\mathbb{P}_{k-1}$ donde $k \leq n$ y satisface [42]

$$
\int_{a}^{b} \pi_{n}(x) p(x) w(x) \mathrm{d} x=0 \quad \forall p \in \mathbb{P}_{k-1} .
$$

Vamos a mostrar que los ceros del polinomio ortogonal son óptimos para esta fórmula interpolatoria y tiene $2 n-1$ grados de exactitud. Por lo que primero dividimos el polinomio $p(x) \in \mathbb{P}_{2 n-1}$ por el polinomio $\pi_{n}(x)$ tal que

$$
p(x)=q(x) \pi_{n}(x)+r(x), \quad q(x), r(x) \in \mathbb{P}_{n-1},
$$

donde $q(x)$ es el cociente y $r(x)$ es el residuo, así tenemos que

$$
\int_{a}^{b} p(x) w(x) \mathrm{d} x=\int_{a}^{b} q(x) \pi_{n}(x) w(x) \mathrm{d} x+\int_{a}^{b} r(x) w(x) \mathrm{d} x,
$$

de donde la primera integral del lado derecho es cero por la condición (4.3.5) ya que $q(x) \in \mathbb{P}_{n-1}$. Como la regla es interpolatoria resulta exacta para $r(x) \in \mathbb{P}_{n-1}$

$$
\int_{a}^{b} p(x) w(x) \mathrm{d} x=\int_{a}^{b} r(x) w(x) \mathrm{d} x
$$




$$
\sum_{k=1}^{N} w_{k} p\left(x_{k}\right)=\sum_{k=1}^{N} w_{k}\left[p\left(x_{k}\right)-q\left(x_{k}\right) \pi_{n}\left(x_{k}\right)\right]=\sum_{k=1}^{N} w_{k} r\left(x_{k}\right)
$$

ya que $\pi_{n}\left(x_{k}\right)=0$ para $k=1,2, \ldots, n$. Por lo tanto los ceros del polinomio ortogonal optimizan la fórmula interpolatoria y la cuadratura tiene una exactitud de $2 n-1$.

La fórmula que se construyó es la llamada cuadratura Gaussiana asociada con la función de peso $w(x)$ en la que los nodos corresponden a los ceros de $\pi_{n}(x)=P_{n}(x ; w)$ y los pesos $w_{k}$ están dados por [42]

$$
\begin{array}{r}
P_{n}\left(x_{k} ; w\right)=0 \quad k=1,2, \ldots, n . \\
w_{k}=\int_{a}^{b} \frac{P_{n}(x ; w)}{\left(x-x_{k}\right) P_{n}^{\prime}\left(x_{k} ; w\right)} w(x) \mathrm{d} x,
\end{array}
$$

La regla de cuadratura es óptima ya que presenta el máximo grado de exactitud con $2 n-1$ usando $n$ nodos.

A partir de lo anterior se pueden establecer algunas características importantes para la regla de cuadratura Gaussiana

- Todos los nodos $x_{k}$ son reales, distintos y están contenidos en el intervalo $(a, b)$ esto cumple con la propiedad para los ceros de polinomios ortogonales.

- Todos los pesos $w_{k}$ son positivos.

- Existen algoritmos eficientes para calcular fórmulas de cuadratura Gaussiana en los que se definen coeficientes de recursión $\alpha_{k}=\alpha_{k}(w)$ y $\beta_{k}=\beta_{k}(w)$ para los polinomios $P_{k}(x)=P_{k}(x ; w)$ que cumplen con la relación de recurrencia de tres términos

$$
\begin{array}{r}
P_{k+1}(x)=\left(x-\alpha_{k}\right) P_{k}(x)-\beta_{k} P_{k-1}(x), \\
P_{0}(x)=1 \quad P_{-1}(x)=0, \quad k=0,1,2, \ldots,
\end{array}
$$

definiendo $\beta_{0}=\int_{a}^{b} w(x) \mathrm{d} x$ que se conoce como primer momento $\mu_{0} \mathrm{y}$ es la medida del intervalo $(a, b)$.

De donde calcular la fórmula Gaussiana consiste en resolver un problema de eigenvalores y eigenvectores para una matriz tridiagonal simétrica correspondiente a la matriz de Jacobi de orden $n\left(\mathbb{J}_{n}\right)$ para la función de peso $w(x)$ [42]. 
Los coeficientes $\alpha_{k}$ y $\beta_{k}$ se definen a partir del producto interno de los polinomios ortogonales

$$
\begin{array}{r}
\alpha_{k}=\frac{\left(P_{k}, x P_{k}\right)}{\left(P_{k}, P_{k}\right)}, \quad k=0,1, \ldots, \\
\beta_{k}=\frac{\left(P_{k}, x P_{k}\right)}{\left(P_{k-1}, P_{k-1}\right)}, \quad k=1,2, \ldots
\end{array}
$$

La matriz de Jacobi de orden $n\left(\mathbb{J}_{n}\right)$ se construye a partir de los coeficientes $\alpha_{k}$ y $\beta_{k}$ con la relación de recurrencia para los polinomios mónicos ortogonales respecto al producto escalar (4.2.1). Los coeficientes $\beta_{k}$ son todos positivos y por lo tanto podemos tomar la raíz cuadrada positiva $\beta_{k}$ de cada uno de ellos.

$$
\mathbb{J}_{n}=\left(\begin{array}{ccccc}
\alpha_{0} & \sqrt{\beta_{1}} & \ldots & 0 & 0 \\
\sqrt{\beta_{1}} & \alpha_{1} & \sqrt{\beta_{2}} & \ldots & 0 \\
\vdots & \ddots & \ddots & \ddots & \vdots \\
0 & \ldots & \sqrt{\beta_{n-2}} & \alpha_{n-2} & \sqrt{\beta_{n-1}} \\
0 & 0 & \ldots & \sqrt{\beta_{n-1}} & \alpha_{n-1}
\end{array}\right),
$$

Así los nodos $x_{k}$ correponden a los valores propios de $\left(\mathbb{J}_{n}\right)$ para la fórmula de cuadratura Gaussiana con $n$ puntos. Los pesos vienen dados por $w_{k}=v_{k, 1}^{2}$ con $k=1,2, \ldots, n$, donde $v_{k, 1}$ es la primer componente del vector propio $v_{k}$.

En la Tabla (4.1) se presentan las funciones de peso clásicas con sus respectivos polinomios ortogonales y coeficientes de recursión que los generan.

Aunque existe una metodología bastante conocida para cuadraturas Gaussianas clásicas el problema que presentamos no se puede resolver directamente usando alguno de los esquemas para polinomios clásicos.

\subsection{Cálculo numérico de integrales con funciones de Bessel y cuadratura Gaussiana en el plano complejo}

En la evaluación numérica de la integral (4.0.7) se podría pensar en utilizar una cuadratura compuesta de Gauss-Legendre transformada para considerar los intervalos entre los nodos del integrando. Sin embargo la convergencia sería lenta dependiendo del valor de $p$. Es posible desarrollar y aplicar cuadraturas Gaussianas específicas con funciones de peso $J_{\nu}(t)(t=p x)$ sobre el intervalo $[0, \infty]$. Sin embargo el problema que aparece está relacionado con la forma usual de definir cuadraturas Gaussianas mediante 


\begin{tabular}{ccccc}
\hline Polinomio ortogonal & $w(x)$ & {$[a, b]$} & $\alpha_{k}$ & $\beta_{k}$ \\
\hline Legendre & 1 & {$[-1,1]$} & 0 & $2(k=0)$ \\
$P_{n}$ & & & $\left(4-k^{-2}\right)^{-1}(k>0)$ \\
Chebyshev tipo 1 & $\left(1-x^{2}\right)^{-1 / 2}$ & {$[-1,1]$} & 0 & $\pi(k=0)$ \\
$T_{n}$ & & & & $\frac{1}{2}(k=1)$ \\
& & & & $\frac{1}{4}(k>1)$ \\
Chebyshev tipo 2 & $\left(1-x^{2}\right)^{1 / 2}$ & {$[-1,1]$} & 0 & $\frac{1}{2} \pi(k=0)$ \\
$U_{n}$ & & & $\frac{1}{4}(k>0)$ \\
Jacobi & $(1-x)^{\alpha}(1+x)^{\beta}$ & {$[-1,1]$} & - & - \\
$P_{n}^{(\alpha, \beta)}$ & $\alpha>-1, \beta>-1$ & & & \\
Laguerre & $x^{\alpha} e^{-x}$ & {$[0, \infty]$} & $2 k+\alpha+1$ & $\Gamma(1+\alpha)(k=0)$ \\
$L_{n}^{(\alpha)}$ & $\alpha>-1$ & & & $\sqrt{\pi}(k=0)$ \\
Hermite & $e^{-x^{2}}$ & {$[-\infty, \infty]$} & 0 & $\frac{1}{2} k(k>0)$ \\
$H_{n}$ & & & &
\end{tabular}

Tabla 4.1: Polinomios ortogonales clásicos [42].

los polinomios ortogonales asociados y su construcción a través de las relaciones de recurrencia de tres términos [44] las cuales se cumplen cuando $w(t) \geq 0$ en el intervalo. Pero la función $J_{\nu}(t)$ no cumple con la condición anterior y además los momentos de orden mayor para la función de peso no existen. En [45] se aborda una generalización de polinomios ortogonales con funciones de peso negativas.

Otra alternativa consiste en formular el problema como un sistema de ecuaciones no lineales cuyas soluciones son los nodos y pesos en donde los momentos de la distribución auxiliar se definen como la integral $\int_{0}^{\infty} t^{\beta+\eta} J_{\nu}(t) e^{-t} \mathrm{~d} t$, así el factor exponencial que aparece permite la convergencia de las integrales. Este problema es difícil ya que implica encontrar la solución de un sistema de $n$ ecuaciones no lineales en dos variables. Además el vector que contiene los pesos y las abscisas puede ser de valor complejo [46], veáse el apéndice $\mathrm{F}$ (15). Los métodos en el plano complejo para la evaluación de este tipo de integrales son de interés y aparecen en [45, 47-49].

Existe la alternativa de transformar la representación integral de la función de Bessel como un problema de una integral doble en el que las cuadraturas de Gauss-Laguerre y Gauss-Chebyshev pueden ser usadas. En este caso las cuadraturas de Gauss-Laguerre permiten transformar al plano complejo [35].

La característica principal en el desarrollo de cuadraturas Gaussianas con funciones de peso $J_{\nu}(t)$ es que la función de Bessel puede tomar valores negativos en el intervalo $[0, \infty]$. En el trabajo de Wong [36] se presenta una 
manera de resolver esta dificultad usando la función de Bessel de primer tipo en términos de las funciones de Hankel

$$
J_{\nu}(t)=\frac{1}{2}\left\{H_{\nu}^{(1)}(t)+H_{\nu}^{(2)}(t)\right\} .
$$

A su vez las funciones de Hankel están definidas en términos de $K_{\nu}(t)$ que son las funciones de Bessel modificadas de tercer tipo

$$
\begin{aligned}
& H_{\nu}^{(1)}(t)=\frac{2}{\pi \imath} e^{-\imath \pi \nu / 2} K_{\nu}(-\imath t) \\
& H_{\nu}^{(2)}(t)=-\frac{2}{\pi \imath} e^{\imath \pi \nu / 2} K_{\nu}(\imath t) .
\end{aligned}
$$

El beneficio es que la función de Bessel modificada del tercer tipo no es negativa en el intervalo $[0, \infty]$. Por lo tanto los polinomios ortogonales y la cuadratura Gaussiana se pueden construir usando $K_{\nu}(x)[x= \pm \imath t]$ como función de peso. Esto conduce a reglas de cuadratura que son de valor real y que sirven para resolver integrales de tipo $I(\eta, \nu, p)=\int_{0}^{\infty} x^{\eta} f(x) J_{\nu}(p x) \mathrm{d} x$ en (4.0.7) que al realizar la sustitución (4.4.1) obtenemos

$$
I(\eta, \nu, p)=\frac{1}{2}\left\{\int_{0}^{\infty} x^{\eta} f(x) H_{\nu}^{(1)}(p x) \mathrm{d} x+\int_{0}^{\infty} x^{\eta} f(x) H_{\nu}^{(2)}(p x) \mathrm{d} x\right\},
$$

donde se debe satisfacer $\eta \pm \nu>-1$ para asegurar la convergencia de las integrales [36]. Usando la transformación $t=p x$ en las dos integrales de (4.4.4) tenemos que

$$
I(\eta, \nu, p)=\frac{1}{2 p^{\eta+1}}\left\{\int_{0}^{\infty} t^{\eta} f(t / p) H_{\nu}^{(1)}(t) \mathrm{d} t+\int_{0}^{\infty} t^{\eta} f(t / p) H_{\nu}^{(2)}(t) \mathrm{d} t\right\}
$$

y sustituyendo las expresiones de las ecuaciones (4.4.2) y (4.4.3)

$$
\begin{array}{r}
I(\eta, \nu, p)=\frac{1}{\imath \pi p^{\eta+1}}\left\{e^{-\imath \pi \nu / 2} \int_{0}^{\infty} t^{\eta} f(t / p) K_{\nu}(-\imath t) \mathrm{d} t-\right. \\
\left.e^{\imath \pi \nu / 2} \int_{0}^{\infty} t^{\eta} f(t / p) K_{\nu}(\imath t) \mathrm{d} t\right\} .
\end{array}
$$

Finalmente reemplazamos $x= \pm \imath t$

$$
\begin{aligned}
& I(\eta, \nu, p)=\frac{1}{\pi p^{\eta+1}}\left\{e^{\imath(\eta-\nu) \pi / 2}\right. \int_{0}^{\infty} x^{\eta} f(\imath x / p) K_{\nu}(x) \mathrm{d} x+ \\
&\left.e^{-\imath(\eta-\nu) \pi / 2} \int_{0}^{\infty} x^{\eta} f(-\imath x / p) K_{\nu}(x) \mathrm{d} x\right\} .
\end{aligned}
$$


Así estas integrales son construidas para cuadraturas Gaussianas con respecto a funciones de peso $x^{\eta} K_{\nu}(x)$ sobre el intervalo $[0, \infty]$. Estas cuadraturas Gaussianas se pueden utilizar para aproximar la integral de una función $g(x)$

$$
\int_{0}^{\infty} g(x) x^{\eta} K_{\nu}(x) d x \approx \sum_{k=1}^{N} g\left(x_{k}\right) w_{k}
$$

donde $x_{k}$ y $w_{k}$ que dependen de $\eta$ y $\nu$ corresponden a las abscisas y pesos de la cuadratura Gaussiana de orden $N$ con función de peso $w(x)=x^{\eta} K_{\nu}(x)$. La fórmula es exacta cuando $g(x)$ es un polinomio de grado $2 N-1$ o menor.

La integral original en la ecuación (4.0.7) puede escribirse como

$$
\begin{array}{r}
I(\eta, \nu, p) \approx \frac{1}{\pi p^{\eta+1}}\left\{e^{\imath(\eta-\nu) \pi / 2} \sum_{k=1}^{N} f\left(\imath x_{k} / p\right) w_{k}+\right. \\
\left.e^{-\imath(\eta-\nu) \pi / 2} \sum_{k=1}^{N} f\left(-\imath x_{k} / p\right) w_{k}\right\},
\end{array}
$$

donde $x_{k}$ y $w_{k}$ son los valores de las abscisas y pesos para la fórmula de cuadratura Gaussiana de orden $N$ basada en la función de peso $x^{\eta} K_{\nu}(x)$. Estas cuadraturas pueden obtenerse de rutinas como el procedimiento de Stieltjes [50,51]. El procedimiento en este caso particular consiste en calcular los respectivos polinomios ortogonales a través de su relación de recurrencia de tres términos (4.3.11)

$$
P_{n+1}(\eta, \nu, x)=\left(x-\alpha_{n}\right) P_{n}(\eta, \nu, x)-\beta_{n} P_{n-1}(\eta, \nu, x),
$$

donde los coeficientes de recursión $\alpha_{n}$ y $\beta_{n}$ se definen como en (4.3.12)

$$
\alpha_{n}=\frac{\int_{0}^{\infty} x P_{n}^{2}(\eta, \nu, x) w(\eta, \nu, x) \mathrm{d} x}{\int_{0}^{\infty} P_{n}^{2}(\eta, \nu, x) w(\eta, \nu, x) \mathrm{d} x}, \quad \beta_{n}=\frac{\int_{0}^{\infty} P_{n}^{2}(\eta, \nu, x) w(\eta, \nu, x) \mathrm{d} x}{\int_{0}^{\infty} P_{n-1}^{2}(\eta, \nu, x) w(\eta, \nu, x) \mathrm{d} x},
$$

$w(\eta, \nu, x)=x^{\eta} K_{\nu}(x)$ es la función de peso del sistema de polinomios ortogonales $\beta_{0}=\int_{0}^{\infty} w(\eta, \nu, x) \mathrm{d} x$ y $P_{0}(x)=1$ mientras que $P_{-1}(x)=0$.

Las integrales correspondientes a los coeficientes de recursión se calculan por la cuadratura de Féjer. Las cuadraturas Gaussianas se obtienen de los coeficientes de recursión por la diagonalización de una matriz tridiagonal de Jacobi $\left(\mathbb{J}_{n}\right)$ (4.3.13) cuyas entradas dependen de los coeficientes de recursión.

Wong [36] examinó las reglas de cuadratura de la función de peso $K_{0}(x)$ para el caso de las integrales con $J_{0}(p x)$ y la función de prueba $f(x)=\frac{1}{(1+x)}$. El método funciona muy bien en las regiones asintóticas 
de $p$ precisamente donde uno encuentra problemas debido a la naturaleza oscilatoria del integrando. Además cabe destacar que los resultados se obtuvieron con cuadraturas de orden bajo $(N=5)$.

La ventaja del procedimiento de Wong es que se consiguen funciones de peso positivas $w(x)=x^{\eta} K_{\nu}(x)$ en el intervalo $[0, \infty]$.

\subsection{Aplicación de la metodología}

Los polinomios ortogonales así como las fórmulas de cuadratura Gaussiana para funciones de peso $K_{0}(x), x K_{1}(x), x^{2} K_{2}(x)$ y $x^{3} K_{3}(x)$ en el intervalo $[0, \infty]$ fueron calculadas para órdenes de $N=5$ hasta $N=70$ usando el procedimiento de Stieltjes discretizado [50,51]. Los coeficientes de recursión así como las cuadraturas para cada función de peso se presentan en el apéndice A (10). Las gráficas de los polinomios ortogonales de primer, segundo y tercer orden para estas funciones de peso se presentan en la Fig. (11.1) del apéndice B (11). Los ceros de estos polinomios corresponden a las abscisas de la respectiva cuadratura Gaussiana de orden $N$.

En el trabajo de Wong [36] sólo se reportan fórmulas de cinco puntos para $K_{0}(x)$, las cuales fueron comparadas y verificadas para demostrar su consistencia. Se han presentado polinomios ortogonales para la función de peso $K_{1 / 3}(x)$ [52].

El algoritmo de Stieltjes requiere de los valores de la función de peso en diferentes valores de $x$. Esto se hizo para $K_{\nu}(x)$ usando la representación integral

$$
K_{\nu}(x)=\int_{0}^{\infty} e^{-x \cosh (t)} \cosh (\nu t) \mathrm{d} t,
$$

se calculó la integral usando una cuadratura estándar de Gauss-Legendre subdividiendo el intervalo de $[0, \infty]$ en subintervalos. Para valores de $x$ pequeños se usó la expansión en serie [37]

$$
\begin{aligned}
K_{\nu}(x)= & \frac{1}{2} \sum_{k=0}^{\nu-1} \frac{(-1)^{k}(\nu-k-1) !}{k !}\left(\frac{x}{2}\right)^{2 k-\nu}+ \\
& \frac{1}{2}(-1)^{\nu-1} \sum_{k=0}^{\infty} \frac{\left(\frac{x}{2}\right)^{2 k+\nu}}{k !(k+\nu) !}\left[2 \log \left(\frac{x}{2}\right)-\psi(k+1)-\psi(k+\nu+1)\right],
\end{aligned}
$$

donde $\psi(x)$ es la derivada logarítmica de la función gamma y la primera suma se omite para $\nu=0$.

Las funciones radiales del átomo de hidrógeno y del oscilador armónico en 2D fueron elegidas para probar la metodología (4.4.9) en esta aplicación 
particular consideramos $x=r$. Así la aproximación (4.4.9) toma la forma

$$
\begin{array}{r}
\widetilde{R}_{n, m}(p) \approx \frac{\imath^{m}}{\pi p^{\eta+1}}\left\{e^{\imath(\eta-\nu) \pi / 2} \sum_{k=1}^{N}\left(\imath r_{k} / p\right) R_{n, m}\left(\imath r_{k} / p\right) w_{k}+\right. \\
\left.e^{-\imath(\eta-\nu) \pi / 2} \sum_{k=1}^{N}\left(-\imath r_{k} / p\right) R_{n, m}\left(-\imath r_{k} / p\right) w_{k}\right\},
\end{array}
$$

donde las $r_{k}$ y $w_{k}$ son las abscisas y pesos de la cuadratura Gaussiana con función de peso $w_{\eta, \nu}(x)$ donde $\eta=\nu=m$. El factor $i r_{k} / p$ en ambas sumas representa el elemento Jacobiano de la integración. 


\section{Objetivos y alcance}

En este trabajo estamos interesados principalmente en:

- Extender y examinar el funcionamiento del método propuesto por Wong para transformar del espacio de posición al espacio de momentos funciones de tipo exponencial y Gaussiano.

- Estudiar el funcionamiento del método cuando se transforma al espacio de momentos en dos dimensiones mediante la transformada de Hankel para el átomo de hidrógeno y el oscilador armónico.

- Introducir una nueva metodología para transformar al espacio de momentos en tres dimensiones mediante la transformada de Bessel esférica en funciones radiales de tipo $f_{n, l}^{S T O}(r)$ y $f_{n, l}^{G T O}(r)$.

- Aplicar la metodología en tres dimensiones para transformar orbitales atómicos al espacio de momentos.

Debemos resaltar que en este trabajo no se va a calcular ninguna integral que tenga un significado físico como en los ejemplos mostrados en la sección (2). Sin embargo, si destacamos la importancia de calcular las funciones en el espacio de momentos $\widetilde{\Psi}(\boldsymbol{p})$ como primer paso para obtener la densidad de momento $I(p)$ ya que una vez calculadas las $\widetilde{\Psi}(\boldsymbol{p})$ no resulta complicado conseguir los momentos $\left\langle p^{k}\right\rangle$ a través de las correspondientes densidades. 


\section{Transformada de Hankel}

En esta parte examinamos el funcionamiento y extendemos la metodología de Wong transformando del espacio de posición al espacio de momentos funciones de tipo exponencial con diferente comportamiento tales como $f_{1}(x)=e^{-x}$ y $f_{2}(x)=e^{-x^{2}}$. Ambas funciones son importantes y aparecen en los modelos del átomo de hidrógeno y el oscilador armónico en 2D. Exploramos el comportamiento del método para valores pequeños de la variable $p$. Estudiamos las propiedades de convergencia en varias regiones de $p$. Analizamos su factibilidad para diferentes órdenes $\nu$ de la función de Bessel correspondientes al número cuántico magnético $|m|$. Finalmente comparamos esta metodología con otro procedimiento.

\section{1 Átomo de hidrógeno en dos dimensiones}

Sabemos que los orbitales hidrogenoides son soluciones de la ecuación de Schrödinger no relativista e independiente del tiempo además forman un conjunto básico que describe el movimiento de un electrón en un campo Coulómbico con un núcleo.

El átomo de hidrógeno en dos dimensiones puede definirse en el contexto de la mecánica cuántica si el movimiento del electrón alrededor del núcleo queda restringido al plano [53]. Para tener una aplicación directa en el mundo físico real ciertas fuerzas tienen que aplicarse para imponer la restricción de planaridad sobre el electrón y el núcleo.

La solución de

$$
H \Psi(x)=E \Psi(x),
$$

se puede obtener en forma cerrada usando coordenadas polares $(r, \phi)$. En este caso se considera una energía potencial $V(r)=\frac{-Z e^{2}}{r}$ al igual que en un sistema unidimensional [54] (en unidades atómicas)

$$
\left[-\frac{\hbar^{2}}{2 m_{e}}\left(\frac{\partial^{2}}{\partial r^{2}}+\frac{1}{r} \frac{\partial}{\partial r}+\frac{1}{r^{2}} \frac{\partial^{2}}{\partial \phi^{2}}\right)-\frac{Z e^{2}}{r}\right] \Psi_{n, m}(r, \phi)=E \Psi_{n, m}(r, \phi),
$$

usando separación de variables se puede escribir

$$
\Psi_{n, m}(r, \phi)=R(r)_{n, m} \Phi_{m}(\phi),
$$

donde para la parte angular tenemos

$$
\Phi_{m}(\phi)=\frac{e^{\imath m \phi}}{\sqrt{2 \pi}}
$$


y para la parte radial

$$
\frac{d^{2}}{d r^{2}} R_{n, m}(r)+\frac{1}{r} \frac{d}{d r} R_{n, m}(r)+\left[\frac{2 m_{e}}{\hbar^{2}}\left(E+\frac{Z e^{2}}{r}\right)-\frac{m^{2}}{r^{2}}\right] R_{n, m}(r)=0,
$$

siendo (6.1.5) la ecuación radial de Schrödinger en dos dimensiones cuya solución $R(r)$ depende sólo de $|m|$. Así la solución (6.1.4) es también la función propia del momento angular a lo largo de la dirección z [55]

$$
\hat{L}_{z}=-\imath \hbar \frac{\partial}{\partial \phi},
$$

que conmuta con el Hamiltoniano por lo que $m$ es un número cuántico adecuado.

La función de onda en coordenadas polares en el espacio de posición es

$$
\Psi_{n, m}(r, \phi)=N_{n, m}\left(2 p_{0} r\right)^{|m|} e^{-p_{0} r} L_{n-|m|-1}^{2|m|}\left(2 p_{0} r\right) e^{\imath m \phi},
$$

donde tenemos que $p_{0}=\frac{2 Z}{2 n-1}$ con $Z$ como la carga nuclear; los números cuánticos $n=1,2,3, \ldots ; m=0, \pm 1, \pm 2, \ldots ; L_{n-|m|-1}^{2|m|}$ el polinomio de Laguerre asociado y la constante de normalización $N_{n, m}=2 p_{0}\left[\frac{(n-|m|-1) !}{2 \pi(2 n-1)[(n+|m|-1) !]^{3}}\right]^{1 / 2}$.

En la Tabla (6.1) mostramos las funciones radiales normalizadas para el átomo de hidrógeno $(Z=1)$ en dos dimensiones $[53,55,56]$ que se usaron para probar la metodología propuesta.

Es importante enfatizar que estamos interesados en aplicar la transformada de Fourier en dos dimensiones (transformada de Hankel) a la función de onda en el espacio de posición (6.1.7).

En [57] se menciona que las funciones de onda en el espacio de momentos son los homólogos a través de la transformada de Fourier de los orbitales en el espacio de posición. Sin embargo, en esta misma referencia aplican la proyección de Fock al átomo de hidrógeno en dos dimensiones y consiguen las eigenfunciones que corresponden a los armónicos esféricos ordinarios en el espacio de momentos

$$
\widetilde{\Psi}_{n, m}\left(p, \phi_{p}\right)=\imath^{m}(-1)^{n-1} \frac{\sqrt{8} p_{0}^{2}}{\left(p_{0}^{2}+p^{2}\right)^{3 / 2}} Y_{n-1, m}\left(\theta_{p}, \phi_{p}\right) .
$$

En este punto debemos considerar que los armónicos esféricos de (6.1.8) tienen expresiones que dependen de senos y cosenos los cuales se representan como [56]

$$
\begin{aligned}
& \sin \theta_{p}=\frac{2 p_{0} p}{\left(p_{0}^{2}+p^{2}\right)}, \\
& \cos \theta_{p}=\frac{\left(p_{0}^{2}-p^{2}\right)}{\left(p_{0}^{2}+p^{2}\right)},
\end{aligned}
$$




\begin{tabular}{cccc}
$n$ & $m$ & $R_{n, m}(r)$ & $\widetilde{R}_{n, m}(p)$ \\
\hline 1 & 0 & $2 e^{-p_{0} r} p_{0}$ & $\frac{4 \sqrt{\frac{2}{\pi}}}{\left(4+p^{2}\right)^{3 / 2}}$ \\
2 & 0 & {$\left[\frac{2}{\sqrt{3}}\right] e^{-p_{0} r} p_{0}\left(1-2 p_{0} r\right)$} & $\frac{12 \sqrt{\frac{6}{\pi}}\left(9 p^{2}-4\right)}{\left(4+9 p^{2}\right)^{5 / 2}}$ \\
2 & \pm 1 & {$\left[\frac{4}{\sqrt{6}}\right] e^{-p_{0} r} p_{0}^{2} r$} & $-\frac{144 \sqrt{\frac{3}{\pi}} p}{\left(4+9 p^{2}\right)^{5 / 2}}$ \\
3 & 0 & {$\left[\frac{1}{\sqrt{5}}\right] e^{-p_{0} r} p_{0}\left(2-8 p_{0} r+4 p_{0}^{2} r^{2}\right)$} & $\frac{20 \sqrt{\frac{10}{\pi}}\left(16-400 p^{2}+625 p^{4}\right)}{\left(4+25 p^{2}\right)^{7 / 2}}$ \\
3 & \pm 1 & {$\left[\frac{4}{\sqrt{30}}\right] e^{-p_{0} r} p_{0}^{2} r\left(3-2 p_{0} r\right)$} & $-\frac{4002 \sqrt{\frac{15}{\pi}} p\left(25 p^{2}-4\right)}{\left(4+25 p^{2}\right)^{7 / 2}}$ \\
3 & \pm 2 & {$\left[\frac{8}{\sqrt{120}}\right] e^{-p_{0} r} p_{0}^{3} r^{2}$} & $-\frac{4000 \sqrt{\frac{15}{\pi}}}{\left(4+25 p^{2}\right)^{7 / 2}}$ \\
4 & 0 & {$\left[\frac{1}{3 \sqrt{7}}\right] e^{-p_{0} r} p_{0}\left(6-36 p_{0} r+36 p_{0}^{2} r^{2}-8 p_{0}^{3} r^{3}\right)$} & $\frac{28 \sqrt{\frac{14}{\pi}}\left(117649 p^{6}-86436 p^{4}+7056 p^{2}-64\right)}{\left(4+49 p^{2}\right)^{9 / 2}}$ \\
4 & \pm 1 & {$\left[\frac{1}{\sqrt{21}}\right] e^{-p_{0} r} p_{0}^{2} r\left(12-16 p_{0} r+4 p_{0}^{2} r^{2}\right)$} & $-\frac{7482 \sqrt{\frac{42}{\pi}} p\left(16-588 p^{2}+2401 p^{4}\right)}{\left(4+49 p^{2}\right)^{9 / 2}}$ \\
4 & \pm 2 & {$\left[\frac{4}{\sqrt{210}}\right] e^{-p_{0} r} p_{0}^{3} r^{2}\left(5-2 p_{0} r\right)$} & $-\frac{10976 \sqrt{\frac{105}{\pi} p^{2}\left(49 p^{2}-4\right)}}{\left(4+49 p^{2}\right)^{9 / 2}}$ \\
4 & \pm 3 & {$\left[\frac{4}{3 \sqrt{35}}\right] e^{-p_{0} r} p_{0}^{4} r^{3}$} & $\frac{153664 i \sqrt{\frac{70}{\pi}} p^{3}}{\left(4+49 p^{2}\right)^{9 / 2}}$ \\
\hline
\end{tabular}

Tabla 6.1: Funciones de onda radiales para el átomo de hidrógeno en dos dimensiones $R_{n, m}(r)$ con $Z=1$ [57]. Las funciones de onda radial en el espacio de momentos son $\widetilde{R}_{n, m}(p)$ [58].

estas dos transformaciones permiten escribir la ecuación (6.1.8) solamente en términos de $p$ y de la eigenfunción del momento angular $e^{\imath m \phi_{p}}$. Por lo tanto es posible conseguir las fórmulas analíticas en el espacio de momentos y así comparar los resultados del método numérico.

Las funciones radiales en el espacio de momentos están relacionadas con el espacio de posición a través de la transformada de Hankel

$$
\widetilde{R}_{n, m}(p)=\imath^{m} \int_{0}^{\infty} R_{n, m}(r) J_{\nu}(p r) r d r .
$$

Las soluciones en forma cerrada de estas integrales están disponibles en [14]. Las representaciones analíticas de las funciones en el espacio de momentos pueden usarse para probar el procedimiento numérico y obtener las transformaciones de las funciones del espacio de posición.

Los resultados obtenidos se analizan y presentan en gráficas que aparecen en la sección (6.3). 


\subsection{Oscilador armónico en dos dimensiones}

El oscilador armónico en dos dimensiones se define a partir de la ecuación de Schrödinger (6.1.1). Considerando un potencial $V(r)=\frac{1}{2} \lambda^{2} r^{2}$ para el oscilador armónico isotrópico tenemos la siguiente ecuación en coordenadas polares

$$
\left[-\frac{\hbar^{2}}{2 m_{e}}\left(\frac{\partial^{2}}{\partial r^{2}}+\frac{1}{r} \frac{\partial}{\partial r}-\frac{1}{r^{2}} \frac{\partial^{2}}{\partial \phi^{2}}\right)+\frac{1}{2} \lambda^{2} r^{2}\right] \Psi_{n, m}(r, \phi)=E \Psi_{n, m}(r, \phi),
$$

podemos realizar la separación de variables como en (6.1.3). Con lo que se obtiene la ecuación diferencial asociada a la parte radial

$$
\left[-\frac{\hbar^{2}}{2 m_{e}}\left(\frac{d^{2}}{d r^{2}}+\frac{1}{r} \frac{d}{d r}-\frac{m^{2}}{r^{2}}\right)+\frac{1}{2} \lambda^{2} r^{2}\right] R_{n, m}(r)=E R_{n, m}(r),
$$

al igual que en la expresión para el átomo de hidrógeno (6.1.5) existe una dependencia con respecto al número cuántico magnético $|m|$. [59]

Resolviendo la ecuación (6.2.2) obtenemos las soluciones normalizadas

$$
\Psi_{n, m}(r, \phi)=N_{n, m} r^{m} e^{-\frac{\lambda r^{2}}{2}} L_{n}^{m}(\lambda r) e^{\imath m \phi},
$$

donde $L_{n}^{m}$ es el polinomio de Laguerre asociado y la respectiva constante de normalización $N_{n, m}=\left[\frac{2 n ! \lambda^{m+1}}{\Gamma(n+m+1)}\right]^{1 / 2}$. Al aplicar la transformada de Fourier a la ecuación (6.2.3) tenemos

$$
\widetilde{\Psi}_{n, m}\left(p, \phi_{p}\right)=N_{n, m} p^{m} e^{-\frac{p^{2}}{2 \lambda}} L_{n}^{m}\left(\frac{p^{2}}{\lambda}\right) Y_{m}\left(\theta_{p}, \phi_{p}\right),
$$

en la que la constante es $N_{n, m}=\left[\frac{2 n ! \lambda^{-m-1}}{\Gamma(n+m+1)}\right]^{1 / 2}$.

Las expresiones analíticas para el oscilador armónico en dos dimensiones se encuentran en la Tabla (6.2).

Los resultados obtenidos se analizan y presentan en gráficas que aparecen en la sección (6.3).

\subsection{Discusión de resultados}

\subsection{1 Átomo de hidrógeno en dos dimensiones}

Los resultados para el átomo de hidrógeno se agrupan y analizan de acuerdo al valor de $m$ ya que controla el orden de la transformada de Hankel. El método utiliza una cuadratura diferente para cada valor de $m$. 


\begin{tabular}{cccc}
$n$ & $m$ & $R_{n, m}(r)$ & $\widetilde{R}_{n, m}(p)$ \\
\hline 0 & 0 & $\sqrt{2} e^{-\beta r^{2}}$ & $\frac{e^{-\frac{p^{2}}{2}}}{\sqrt{\pi}}$ \\
1 & 1 & $r e^{-\beta r^{2}}\left(2-r^{2}\right)$ & $\frac{e^{-\frac{p^{2}}{2}} p\left(2-p^{2}\right)}{2 \sqrt{2 \pi}}$ \\
1 & 2 & {$\left[\frac{r^{2}}{\sqrt{3}}\right] e^{-\beta r^{2}}\left(3-r^{2}\right)$} & $\frac{e^{-\frac{p^{2}}{2}} p^{2}\left(3-p^{2}\right)}{\sqrt{6 \pi}}$ \\
2 & 0 & {$\left[\frac{\sqrt{2}}{2}\right] e^{-\beta r^{2}}\left(2-4 r^{2}+r^{4}\right)$} & $\frac{e^{-\frac{p^{2}}{2}}\left(2-4 p^{2}+p^{4}\right)}{2 \sqrt{\pi}}$ \\
2 & 3 & {$\left[\frac{r^{3}}{2 \sqrt{30}}\right] e^{-\beta r^{2}}\left(20-10 r^{2}+r^{4}\right)$} & $\frac{e^{-\frac{p^{2}}{2}} p^{3}\left(20-10 p^{2}+p^{4}\right)}{4 \sqrt{15 \pi}}$ \\
3 & 1 & {$\left[\frac{r}{\sqrt{2}}\right] e^{-\beta r^{2}}\left(24-36 r^{2}+12 r^{4}-r^{6}\right)$} & $\frac{e^{-\frac{p^{2}}{2}} p\left(24-36 p^{2}+12 p^{4}-p^{6}\right)}{12 \sqrt{\pi}}$ \\
3 & 2 & {$\left[\frac{r^{2}}{6 \sqrt{10}}\right] e^{-\beta r^{2}}\left(60-60 r^{2}+15 r^{4}-r^{6}\right)$} & $\frac{e^{-\frac{p^{2}}{2}} p^{2}\left(60-60 p^{2}+15 p^{4}-p^{6}\right)}{12 \sqrt{5 \pi}}$ \\
4 & 0 & {$\left[\frac{\sqrt{2}}{24}\right] e^{-\beta r^{2}}\left(24-96 r^{2}+72 r^{4}-16 r^{6}+r^{8}\right)$} & $\frac{e^{\frac{p^{2}}{2}}\left(24-96 p^{2}+72 p^{4}-16 p^{6}+p^{8}\right)}{24 \sqrt{\pi}}$ \\
4 & 3 & {$\left[\frac{r^{3}}{24 \sqrt{105}}\right] e^{-\beta r^{2}}\left(840-840 r^{2}+252 r^{4}-28 r^{6}+r^{8}\right)$} & $\frac{e^{-\frac{p^{2}}{2}} p^{3}\left(840-840 p^{2}+252 p^{4}-28 p^{6}+p^{8}\right)}{24 \sqrt{210 \pi}}$ \\
\hline
\end{tabular}

Tabla 6.2: Funciones de onda radiales para el oscilador armónico en dos dimensiones $R_{n, m}(r)$ donde $\beta=\frac{\lambda}{2}$ con $\lambda=1$. Las funciones de onda radial en el espacio de momentos son $\widetilde{R}_{n, m}(p)$ [58].

Las gráficas se dividen para analizar el funcionamiento del método en dos intervalos llamados la región asintótica $1 \leq p \leq 50$ y la región no asintótica $0<p \leq 1$. Estudiamos la convergencia del método con respecto al orden de la cuadratura $N$ y los valores de $p$ en la región no asintótica. También se presentan las gráficas de una función por cada valor de $m$. Además tenemos que los resultados y el comportamiento son representativos para otras funciones con el mismo valor de $m$.

Usamos la metodología de Wong para calcular numéricamente las integrales que involucran la transformación de funciones radiales en el espacio de momentos empleando un código escrito en FORTRAN. Se calcularon las integrales para funciones radiales con $m=0, m=1, m=2$ y $m=3$ utilizando aritmética en precisión doble para diferentes valores de la variable $p$ y comparamos con los valores que se obtienen a partir de las expresiones analíticas presentes en la Tabla (6.1). Las gráficas se generaron calculando valores con pasos de una unidad en la región $1 \leq p \leq 50$ y 0.02 unidades en la región $0<p \leq 1$. Graficamos el logaritmo base 10 del valor absoluto del error relativo $\log _{10}[$ Error $]$ contra los valores de $p$, donde definimos al Error $=\left|\frac{v_{n}-v_{a}}{v_{a}}\right|$ con $v_{n}$ y $v_{a}$ como el valor numérico y el valor analítico respectivamente. 


\subsubsection{1 $m=0$}

En la Fig. (6.1) se muestra el error como una función de $p$ la cual se obtiene a partir de las cuadraturas de orden $N=5$ y $N=10$ basadas en la función de peso $K_{0}(r)$. Las gráficas del cálculo de $\widetilde{R}_{4,0}(p)$ muestran que el método funciona mejor (el error disminuye) a medida que $p$ aumenta para un orden de cuadratura particular. Resaltamos que se pueden obtener buenos resultados para $p$ mayor con tan sólo una cuadratura de cinco puntos lo cual está de acuerdo con la observación de Wong [36].

El método funciona mejor en regiones grandes del valor de $p$ donde los métodos convencionales tienen problemas debido a la naturaleza oscilatoria del integrando. La cuadratura de diez puntos produce resultados esencialmente exactos para $p>2$.

El mejor funcionamiento del método para $p$ grande con una cuadratura de orden inferior significa que el integrando debe comportarse como un polinomio de orden inferior para $p$ grande. Lo anterior se puede entender a partir de (4.5.2) donde $\widetilde{R}_{4,0}(p)$ es una función de valor real por lo que los componentes imaginarios de la ecuación (4.5.2) deben cancelarse. El comportamiento de la componente real del integrando en el primer término para diferentes valores de $p$ se presenta en la Fig. (6.2). Se observa que el comportamiento oscilatorio del integrando para $p$ menor se amortigua a medida que $p$ aumenta de hecho la función se asemeja a un polinomio de orden inferior para $p$ grande. El integrando del segundo término de la ecuación (4.5.2) muestra el mismo comportamiento. 


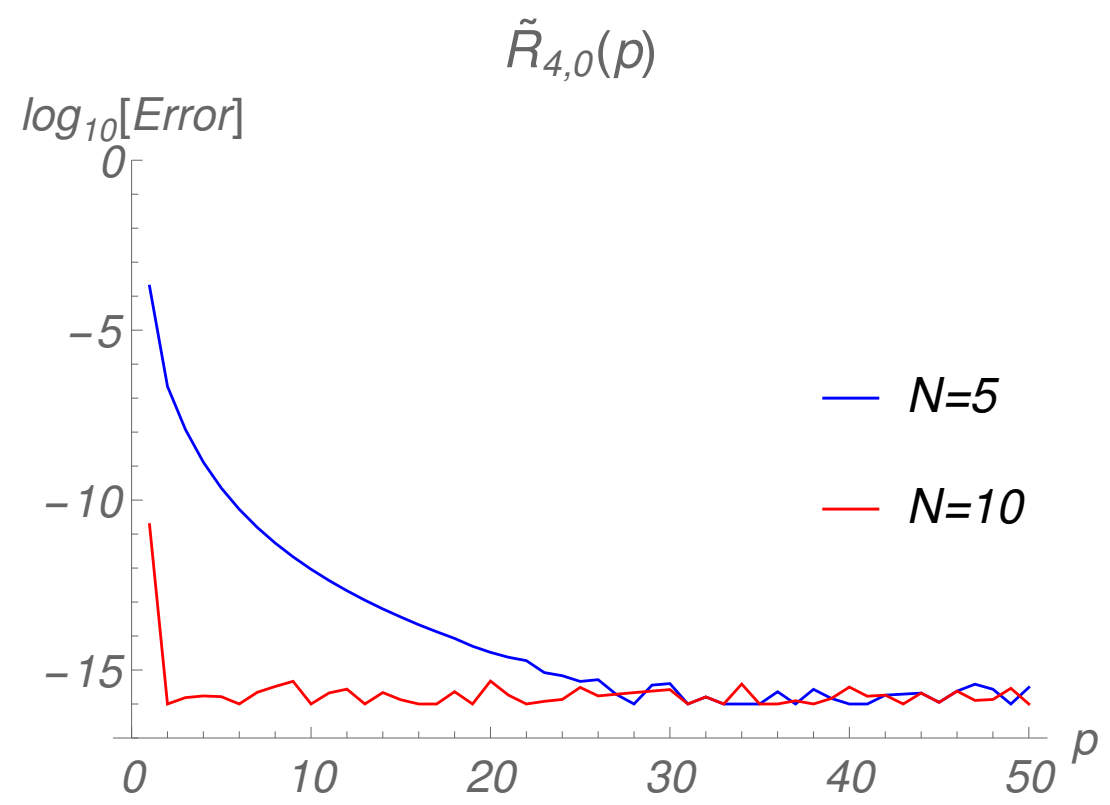

Figura 6.1: Gráfica del logaritmo del error relativo contra el valor de $p$ para funciones radiales con $m=0$ en la región de $1.0-50.0$ en $p$.

La comparación de las curvas de la Fig. (6.1) muestra que se pueden obtener mejores resultados para un valor particular de $p$ aumentando el orden de la cuadratura. Esto es evidente en las regiones más pequeñas de $p$. Una característica importante del método es que el error aumenta al disminuir $p$. Uno de los objetivos principales de este trabajo es determinar si se pueden obtener resultados confiables con este método en esta región. 

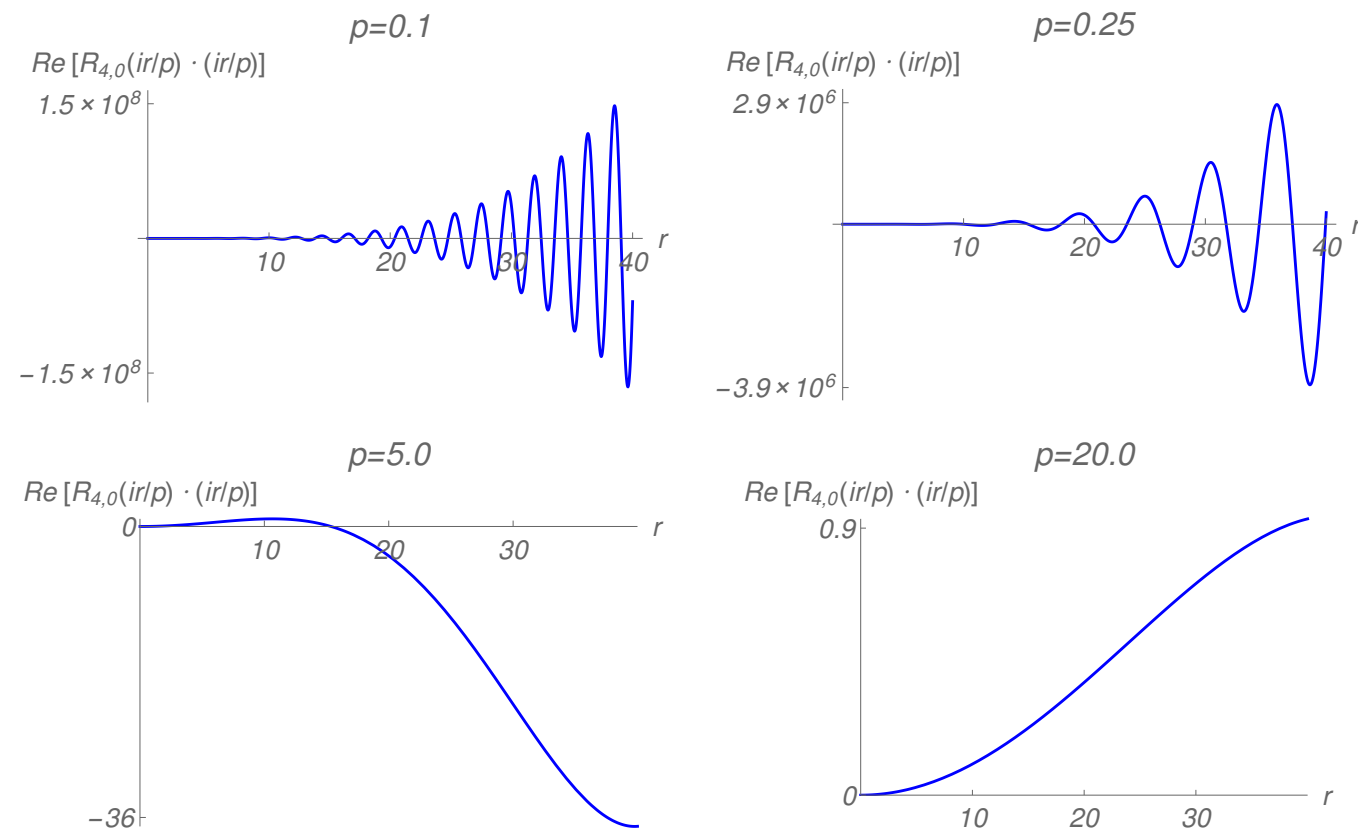

Figura 6.2: Gráfica de $\operatorname{Re}\left[R_{4,0}(\imath r / p) \cdot(\imath r / p)\right]$ contra $r$ para diferentes valores de $p$.

La Fig. (6.3) presenta el análisis del error con diferentes órdenes de cuadratura para $0<p \leq 1$. Se observa que cuadraturas de orden superior tienen que ser empleadas en esta región con el fin de alcanzar resultados razonables. Por lo que se obtienen mejores resultados aumentando el orden de la cuadratura.

Un estudio del error frente al orden de la cuadratura para $p=0.2$ se muestra en la Fig. (6.4). Vemos que pueden conseguirse resultados confiables aumentando el orden de la cuadratura, sin embargo, se enfatiza que el método falla para valores menores de $p$. 


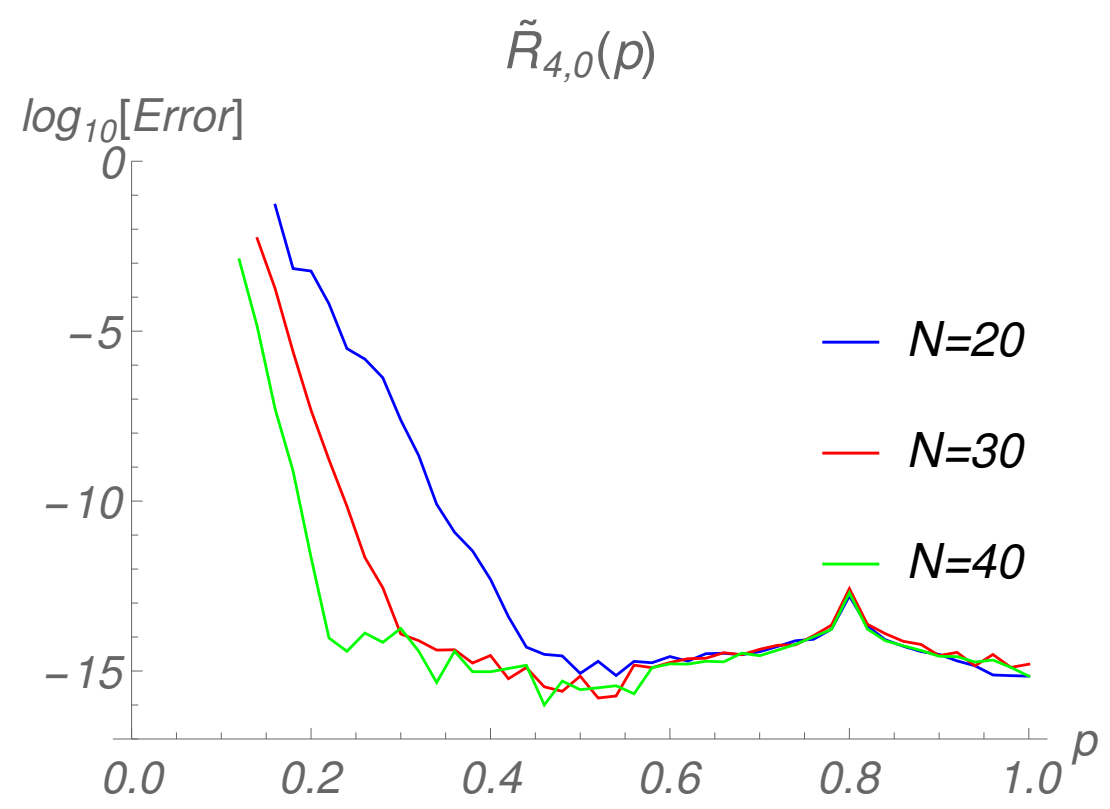

Figura 6.3: Gráfica del logaritmo del error relativo contra el valor de $p$ para funciones radiales con $m=0$ en la región de $0.0-1.0$ en $p$.

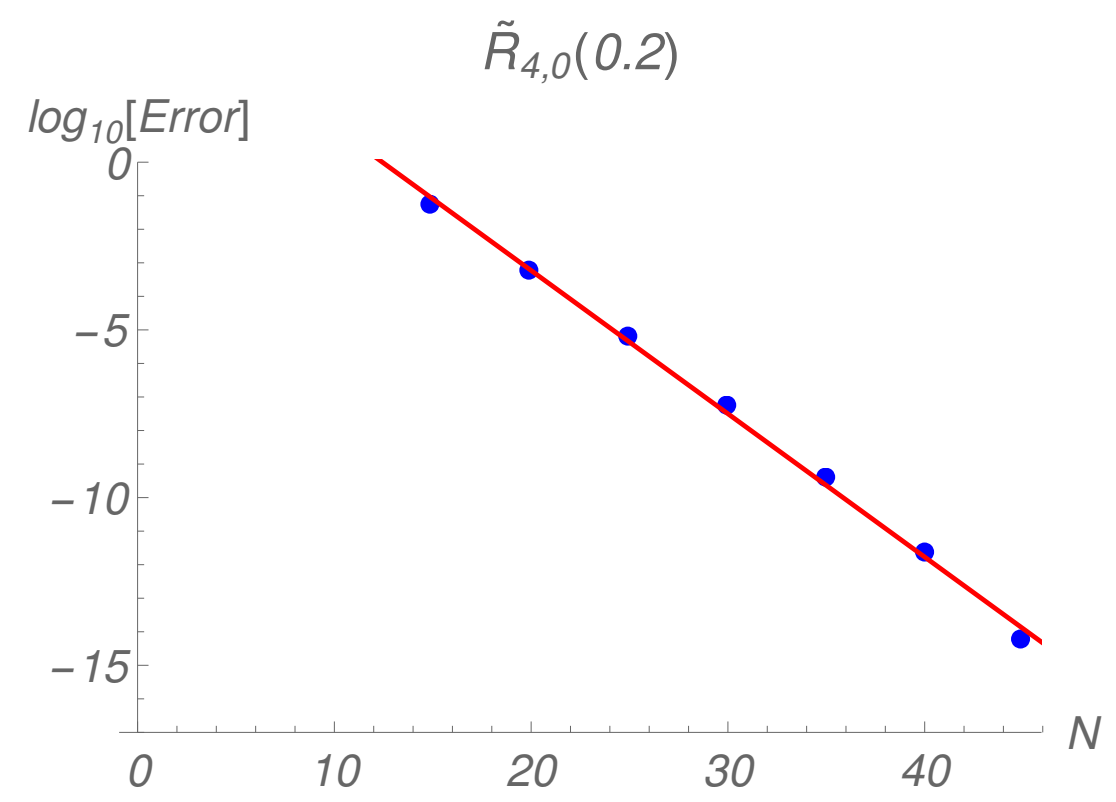

Figura 6.4: Gráfica del logaritmo del error relativo contra el orden de la cuadratura $N$ para la función con $m=0$ en $p=0.2$. 


\subsubsection{2 $m=1$}

En esta sección se estudia el funcionamiento de las cuadraturas basadas en la función de peso $r K_{1}(r)$. Es importante señalar que graficamos el error en los valores de la norma o densidades para la función de tipo $m=1$, es decir, $\left|\widetilde{R}_{4,1}(p)\right|^{2}$ ya que la función en sí es de valor complejo.

La Fig. (6.5) presenta las gráficas para la función $m=1$ donde se observan resultados similares a los de la función $m=0$. La cuadratura de cinco puntos produce valores esencialmente exactos para $p$ grandes del mismo modo la cuadratura de diez puntos produce resultados exactos en el intervalo $2<p \leq 50$. Una observación adicional para la gráfica $N=5$ es que esta cuadratura logra resultados exactos a valores más pequeños de $p$ en comparación con las cuadraturas $K_{0}(r)$ de cinco puntos para $m=0$ en la Fig. (6.1).

Los resultados para valores pequeños de $p(0<p \leq 1)$ se presentan en la Fig. (6.6). Obtenemos excelentes resultados con una cuadratura de $N=40$ hasta $p \approx 0.2$ y no se pueden conseguir resultados significativos para $p<0.2$ con las cuadraturas estudiadas. Por otro lado la Fig. (6.7) para $p=0.2$ ilustra que los resultados se pueden mejorar incrementando el orden de la cuadratura.

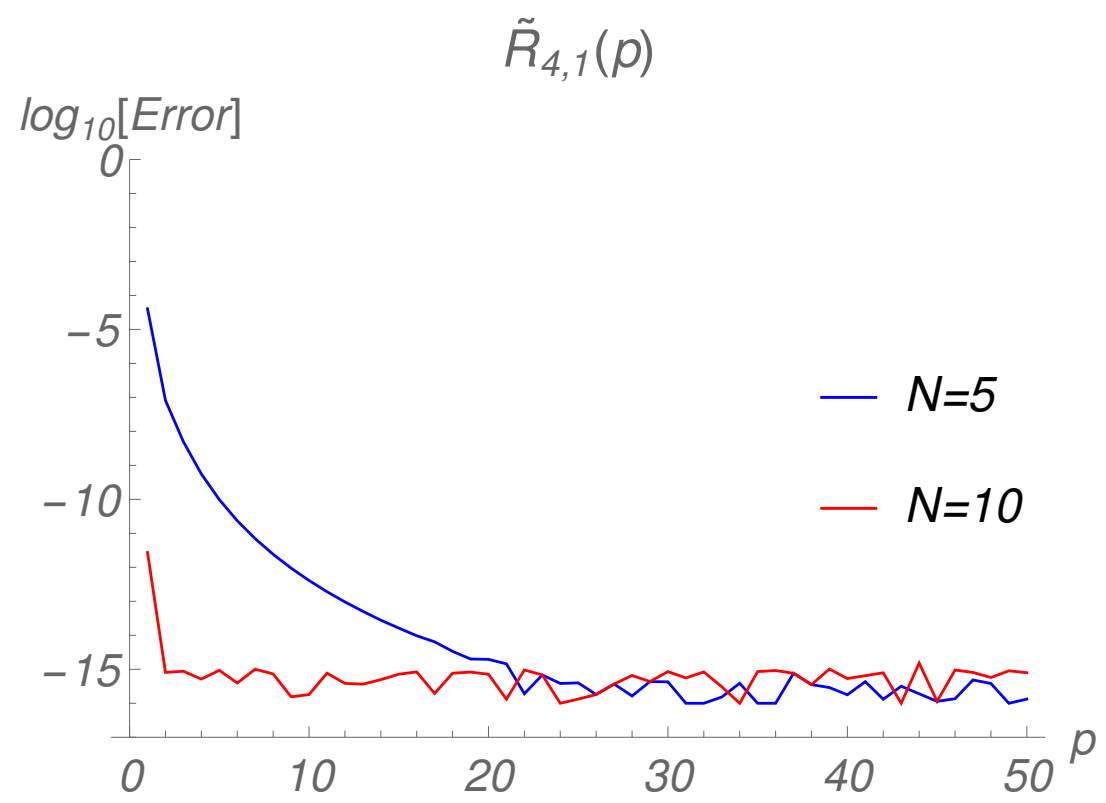

Figura 6.5: Gráfica del logaritmo del error relativo contra el valor de $p$ para funciones radiales con $m=1$ en la región de $1.0-50.0$ en $p$. 


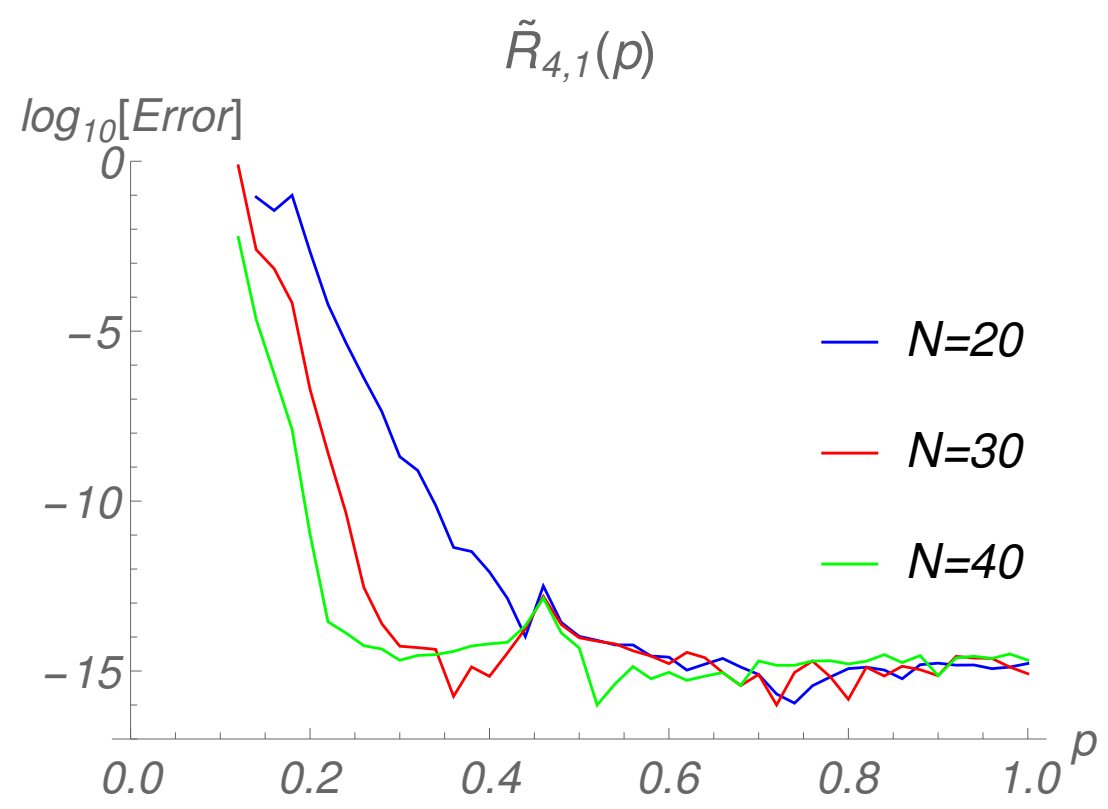

Figura 6.6: Gráfica del logaritmo del error relativo contra el valor de $p$ para funciones radiales con $m=1$ en la región de $0.0-1.0$ en $p$.

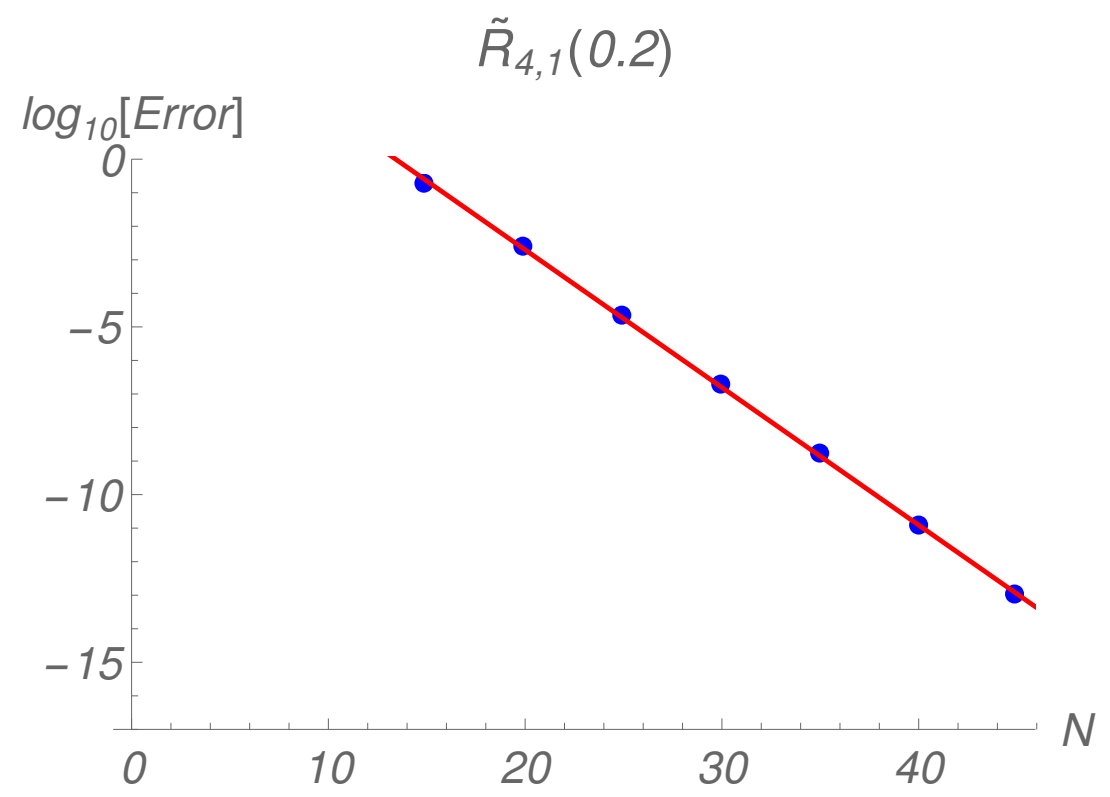

Figura 6.7: Gráfica del logaritmo del error relativo contra el orden de la cuadratura $N$ para la función con $m=1$ en $p=0.2$. 


\subsubsection{3 $m=2,3$}

Los resultados obtenidos de las cuadraturas basadas en la función de peso $r^{3} K_{3}(r)$ se presentan en las Figs. $(6.8-6.10)$. Debemos tomar en cuenta que el error en la norma y no el error en la función es el que se representa para $\left|\widetilde{R}_{4,3}(p)\right|^{2}$. También se estudiaron las gráficas pertenecientes a los valores de las cuadraturas con la función de peso $r^{2} K_{2}(r)$ pero no se presentan. Las gráficas muestran un comportamiento similar a lo visto para $m=3$.

Los resultados son semejantes a los presentados en $m=0,1$. La cuadratura de cinco puntos produce valores esencialmente exactos para $p>20$ mientras que la cuadratura de diez puntos da un excelente resultado en el intervalo de $1 \leq p \leq 50$. La cuadratura en orden $N=40$ proporciona buenas aproximaciones para $p>0.2$. El error aumenta rápidamente con $p<0.2$ por lo que al método le cuesta proporcionar valores precisos en esta región.

La Fig. (6.10) muestra la convergencia del método con el orden de cuadratura en $p=0.2$ lo cual demuestra que se pueden obtener mejores resultados aumentando el orden de la cuadratura.

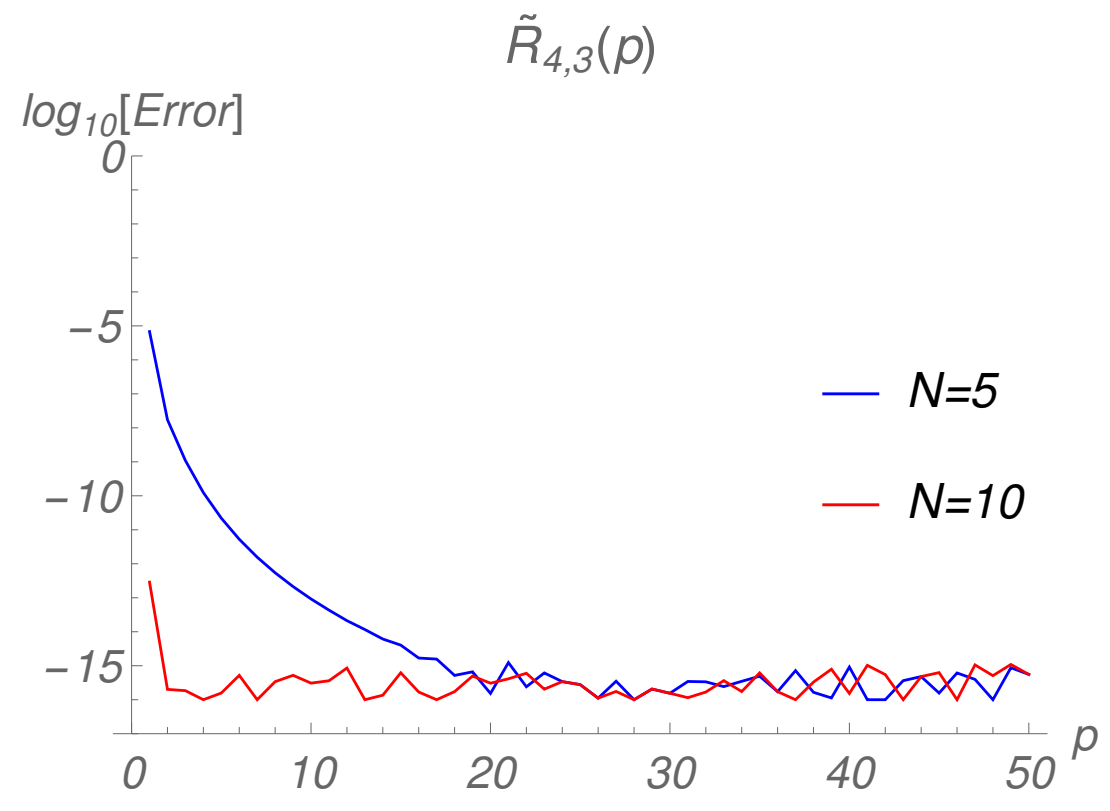

Figura 6.8: Gráfica del logaritmo del error relativo contra el valor de $p$ para funciones radiales con $m=3$ en la región de $1.0-50.0$ en $p$. 


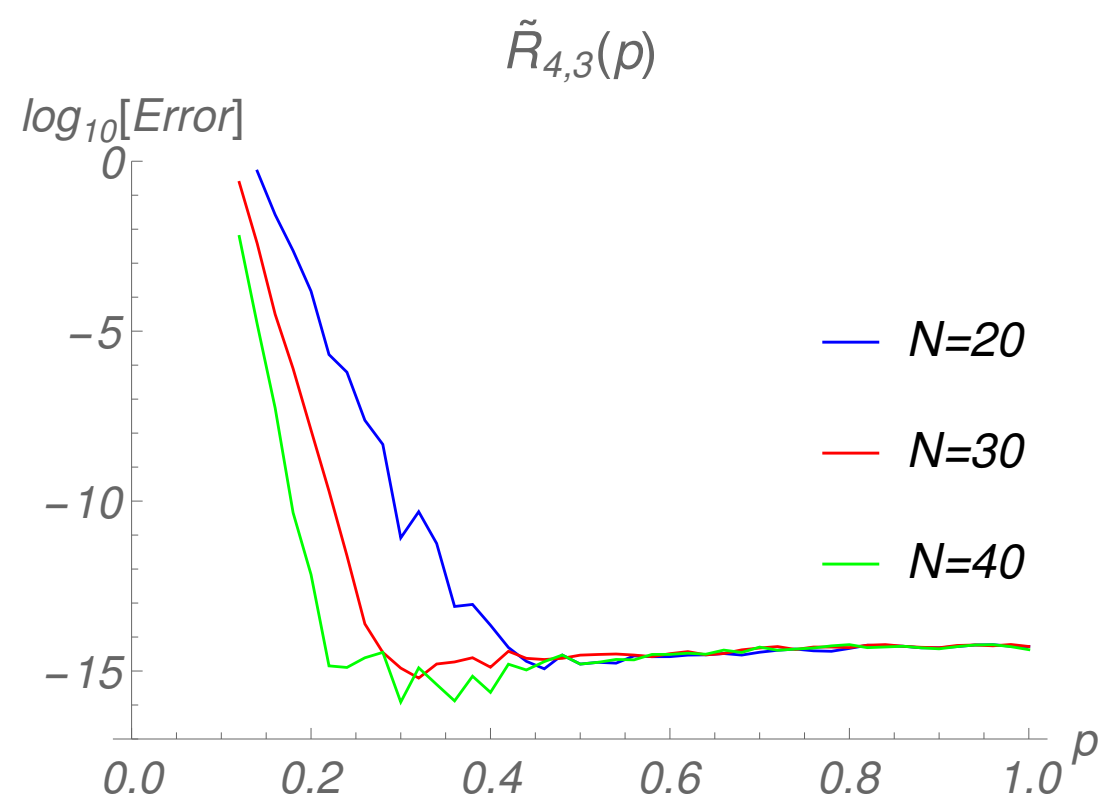

Figura 6.9: Gráfica del logaritmo del error relativo contra el valor de $p$ para funciones radiales con $m=3$ en la región de $0.0-1.0$ en $p$.

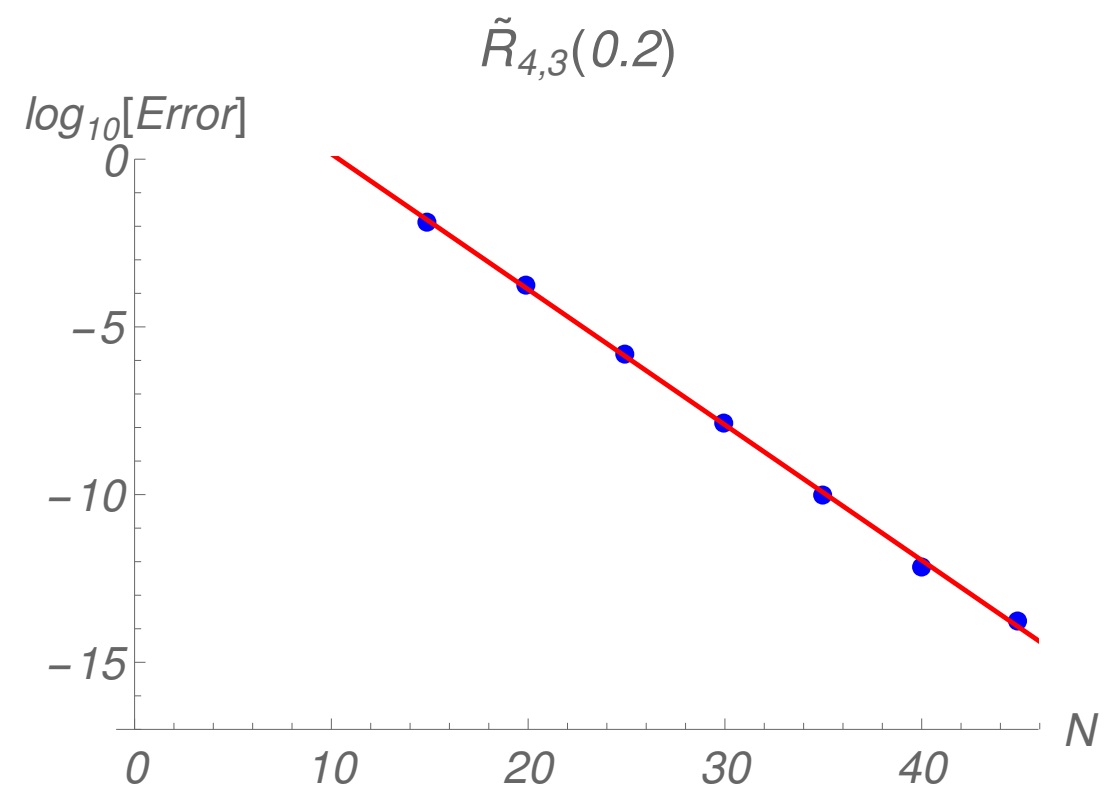

Figura 6.10: Gráfica del logaritmo del error relativo contra el orden de la cuadratura $N$ para la función con $m=3$ en $p=0.2$. 


\subsubsection{Comportamiento del método para valores de $Z>1$}

En esta parte mostramos como cambia el comportamiento del método cuando se modifican los valores de $Z$. Para demostrar esto usamos la función de prueba $\widetilde{R}_{1,0}(p)$ con $m=1$ y $w(r)=K_{0}(r)$. En la Fig (6.11) se observa que al incrementar el valor de $Z$ al método le cuesta conseguir buenos resultados en la región para valores de $p$ pequeños. Esto es evidente con el valor de $Z=20$ ya que al compararse contra el valor de $Z=1$ se muestra que la diferencia es notable aun incrementando el orden de la cuadratura. No se consiguen buenos resultados para $p \leq 10$ al incrementar el orden de la cuadratura $N$ en $Z=20$.

Mostramos que conforme crece el valor de $Z$ se requieren órdenes de cuadratura cada vez mayores para conseguir valores en la región no asintótica de $p$. Lo anterior indica que el método funciona muy bien para valores de $Z$ pequeños usando cuadraturas de orden bajo.

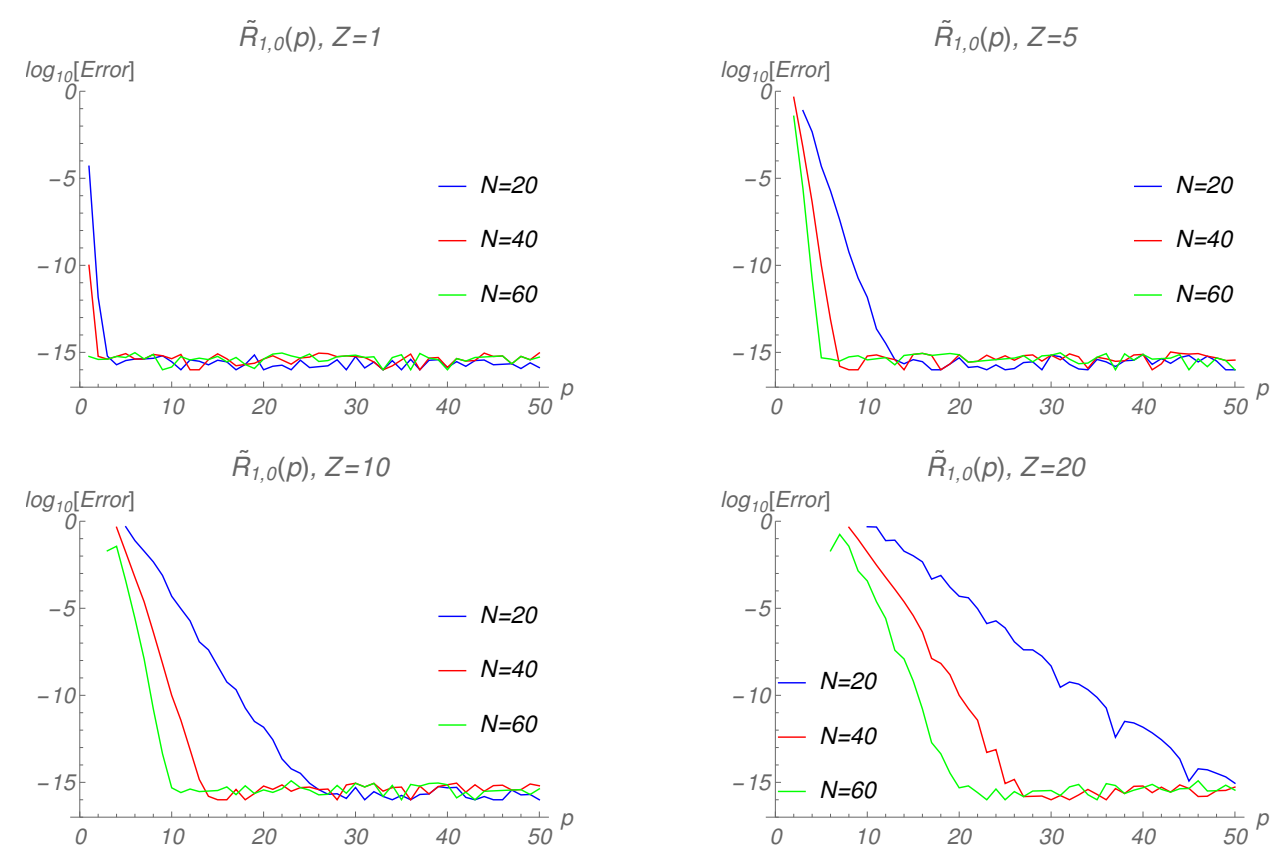

Figura 6.11: Gráfica del logaritmo del error relativo contra el valor de $p$ para la función radial $\widetilde{R}_{1,0}(p)$ con diferentes valores de $Z=1,5,10,20$ en la región de $1.0-50.0$ en $p$. 


\subsection{Oscilador armónico en dos dimensiones}

La metodología de Wong falla al calcular la transformada de Hankel. Por esta razón se realiza un estudio para identificar por qué el método no funciona en la aplicación del oscilador armónico.

Este resultado se puede entender al considerar la ecuación (4.5.2) donde se observa que en todas las funciones de peso consideradas $\left[K_{0}(r), r K_{1}(r)\right.$, $\left.r^{2} K_{2}(r), r^{3} K_{3}(r)\right] \eta=\nu$ por lo que los factores de fase que preceden a las sumas de la ecuación (4.5.2) son iguales a uno en todos los casos. La inspección de la Tabla (6.2) muestra que los factores $r^{m}$ en las funciones radiales se incorporan a las funciones de peso de la cuadratura gaussiana $(\eta=m)$ y las restantes funciones gaussianas multiplicadas por polinomios que son funciones pares. Pero también hay otro factor de $r$ a considerar que viene del elemento Jacobiano de la integración. La multiplicación de una función par por $r$ conduce a una función impar donde $f(-r)=-f(r)$. Al sustituir tales funciones en la ecuación (4.5.2) da como resultado que cada término de la segunda suma sea el negativo del término correspondiente en la primera suma esto conduce a la cancelación y por lo tanto el valor da cero.

También se puede considerar la incorporación del factor $r$ del elemento de integración a la función de peso de la cuadratura Gaussiana es decir $r^{m+1} K_{m}(r)$. Los factores de fase de la ecuación (4.5.2) ahora son $+\imath \mathrm{y}-\imath$ respectivamente. Las funciones radiales a integrar son ahora pares y cada término (valor real) de la primera suma es igual al término correspondiente de la segunda. Sin embargo, el primer término se multiplica por $+\imath$ mientras que el segundo con $-\imath$ que conduce a la cancelación y por lo tanto también da un valor de cero. Se debe considerar que existen restricciones en el tipo de partición de las funciones de peso que se pueden considerar debido a $\eta \pm \nu>-1$.

El método aplicado a la función Gaussiana da cero para la integral (4.5.2) independientemente del valor de $p$ esto puede analizarse examinando la ecuación (4.4.9). Debido a los valores de $\eta$ y $\nu$ los prefactores de cada suma en la ecuación (4.4.9) dan términos iguales con signos contrarios. Por lo tanto para una función que satisface $f\left(-\imath r_{k} / p\right)=f\left(\imath r_{k} / p\right)$ la primera suma se cancela con la segunda y de esto tenemos un resultado de cero en la transformada de Hankel.

Veamos lo anterior considerando la función del oscilador armónico como $f_{1,0}(r)=c e^{-\frac{\alpha r^{2}}{2}}$. Entonces a partir de la ecuación (4.4.7) para $n=1$ con $m=0$ tenemos que $\eta=1$ y $\nu=0$ ya que la transformada de Hankel tiene un factor de $r$ en la representación integral. Para este caso el factor de peso 
es $w(r)=r K_{0}(r)$ de donde

$$
\begin{aligned}
I(p) & =\frac{1}{\pi p^{2}}\left\{e^{\imath \pi / 2} \int_{0}^{\infty} f(\imath r / p) r K_{0}(r) \mathrm{d} r+e^{-\imath \pi / 2} \int_{0}^{\infty} f(-\imath r / p) r K_{0}(r) \mathrm{d} r\right\} \\
& =\frac{\imath}{\pi p^{2}}\left\{\int_{0}^{\infty} f(\imath r / p) w(r) \mathrm{d} r-\int_{0}^{\infty} f(-\imath r / p) w(r) \mathrm{d} r\right\} \\
& =\frac{c \imath}{\pi p^{2}}\left\{\int_{0}^{\infty} e^{-\frac{\alpha(\imath r / p)^{2}}{2}} w(r) \mathrm{d} r-\int_{0}^{\infty} e^{-\frac{\alpha(-\imath r / p)^{2}}{2}} w(r) \mathrm{d} r\right\} \\
& =\frac{c \imath}{\pi p^{2}}\left\{\int_{0}^{\infty} e^{\frac{\alpha r^{2}}{2 p^{2}}} w(r) \mathrm{d} r-\int_{0}^{\infty} e^{\frac{\alpha r^{2}}{2 p^{2}}} w(r) \mathrm{d} r\right\} \\
& =0 .
\end{aligned}
$$

Lo mismo se observa para los casos en que $w(r)$ es igual a $K_{0}(r)$ y $r K_{1}(r)$.

Una estrategia que podría resolver el problema consiste en romper la simetría que provoca la cancelación de términos en (4.4.7) mediante la introducción de un factor como $e^{\alpha r} e^{-\alpha r}$ (multiplicar por uno). Esto funcionaría si fuera posible incorporar el factor $e^{-\alpha r}$ dentro de la función de peso. Pero al no existir una expresión en términos de funciones elementales (exponenciales) para las funciones de Bessel de orden entero [37] no se puede eliminar la simetría. Sin embargo, en el caso de las funciones de Bessel esféricas si es posible introducir este factor.

Otra función problemática es $f(r)=\frac{r}{\left(1+r^{2}\right)^{3 / 2}}$ estudiada por Linz [23] y tratada en [35] que sin estar relacionada con la aplicación de las transformadas integrales también falla cuando se calcula usando el método de Wong.

Algo interesante es que el método funciona bien en la región asintótica $(p \geq 10)$. Para expresiones de tipo Gaussiano en las que no se aplica la transformada de Hankel, es decir en integrales donde el término $r^{\eta}=1$ siendo la función de peso $w(r)=K_{0}(r)$. En la Fig. (6.12) se muestran algunos resultados numéricos que confirman lo anterior. Además tenemos que al sustituir en la ecuación (4.4.9) el valor de la integral $I(p)$ es diferente de cero.

El problema en la aplicación de la metodología al oscilador armónico es el elemento Jacobiano de la integración en la transformación de Hankel. Aunque el método puede aplicarse a una integral con función general de Bessel la Fig. (6.12) muestra los resultados para los cálculos de $\int_{0}^{\infty} f(r) J_{0}(p r) \mathrm{d} r$ con $f(r)=e^{-r^{2}}$. Observamos que el método funciona bien en las regiones de $p$ más grandes asimismo se pueden obtener resultados más precisos usando cuadraturas de orden superior pero no es posible conseguir resultados significativos para $p$ cercanas a cero. En realidad hay regiones en 
$p$ donde la cuadratura de orden inferior produce mejores resultados que la de orden superior y aparecen problemas con la representación de la máquina para las cuadraturas de orden superior.

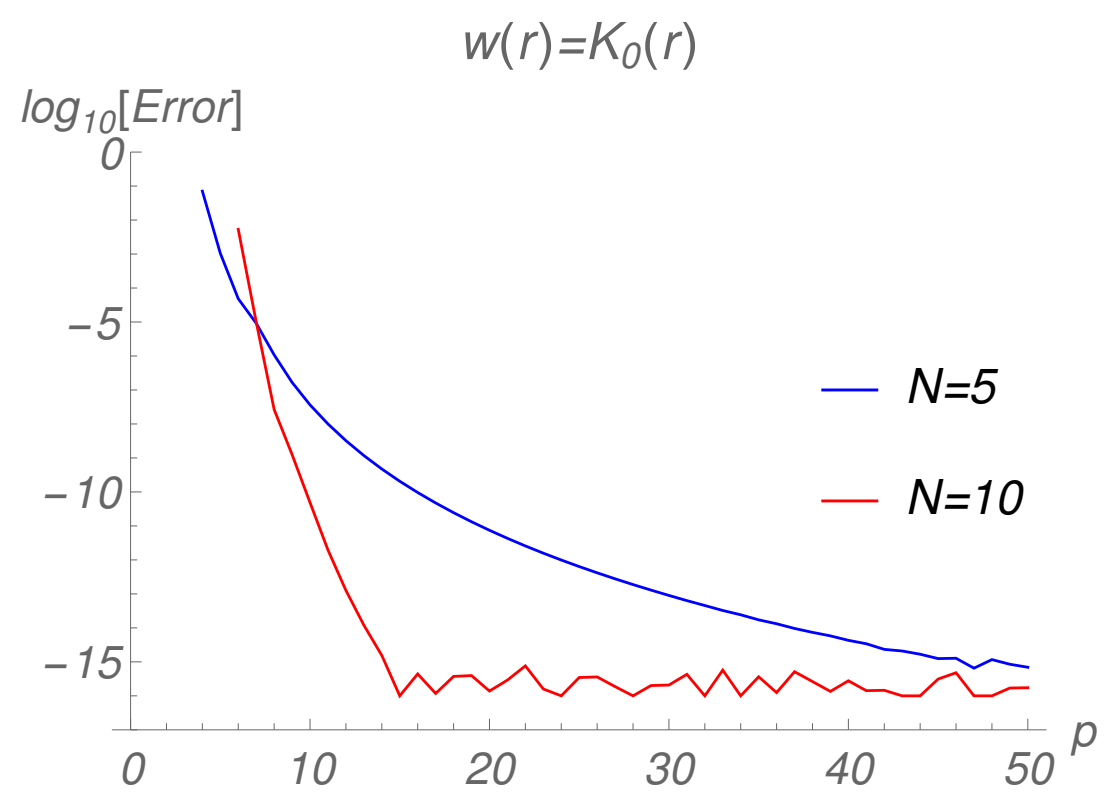

Figura 6.12: Gráfica del logaritmo del error relativo contra el valor de $p$ para la función Gaussiana sin transformar $f(r)=e^{-r^{2}}$ utilizando la función de peso $w(r)=K_{0}(r)$ en la región de $1.0-50.0$ en $p$.

\subsection{Comparación con otra metodología}

A continuación se compara este método con otra metodología que se desarrolla en [14] como una alternativa para resolver el problema de calcular numéricamente integrales dadas por la ecuación (4.0.7).

Este procedimiento numérico calcula integrales que tienen la función de Bessel y en donde aparecen funciones con comportamiento de tipo exponencial. El método utiliza la representación integral de la función de Bessel para reformular el problema como una integral doble. En donde una de las integrales se calcula con la cuadratura de Gauss-Chebyshev mientras que la otra integral utiliza una cuadratura de Gauss-Laguerre dependiente de un parámetro en el plano complejo [35]. Por lo anterior se espera que esta metodología sea exacta cuando se calcule una función exponencial como $f(r)=e^{-r}$.

Se debe considerar que al tratarse de una integral doble cada integral 
requiere de un orden cuadratura diferente para poder calcularse. Por esta razón se establecen dos órdenes de cuadratura $N$ y $M$ tales que $(N, M)=$ $N \times M$ donde para obtener el número total de puntos necesarios para calcular la integral original se multiplican los órdenes de ambas cuadraturas. La cuadratura de Gauss-Chebyshev para la primera integral es de orden $N=25$ y la cuadratura de Gauss-Laguerre para la segunda integral es de orden $M=50$, lo cual da $N \times M=1250$ puntos de evaluación total para calcular la integral.

La segunda integral requiere la introdución de un parámetro $\alpha$ que se utiliza para modelar el comportamiento exponencial de la función. Para esta prueba se selecciona un valor del parámetro $\alpha=20$ de acuerdo a [35].

En este caso no estamos usando la transformada de Hankel para transformar funciones. Lo que hacemos es calcular las integrales asociadas con la función de Bessel y la función de prueba seleccionada considerando integrales de la forma (4.0.7). Comparamos con respecto al $\log _{10}[$ Error $]$ en ambos métodos.

En la Tabla (6.3) se muestra el comparativo para una función con comportamiento de tipo exponencial $f(r)=\frac{1-e^{-5 r}}{r}$. Se selecciona esta función de prueba para que pueda ser comparado el cálculo con el método de Wong. Por lo mencionado arriba si se tomará solamente una función exponencial por construcción sería exacta usando el método de Luna et al.

\begin{tabular}{ccccc}
\hline$p$ & $\begin{array}{c}\text { Luna } \text { et al. }(\alpha=20) \\
(N \times M)=1250\end{array}$ & $\log _{10}[$ Error $]$ & $\begin{array}{c}\text { Wong } \\
N=15\end{array}$ & $\log _{10}[$ Error $]$ \\
\hline 0.5 & 2.99822295 & -9.8 & 2.38919972 & -0.6 \\
& 2.99822295 & & 2.99822295 & \\
1.0 & 2.31243834 & -9.7 & 2.27666191 & -1.8 \\
& 2.31243834 & & 2.31243834 & \\
2.0 & 1.64723115 & -9.5 & 1.64725797 & -4.7 \\
& 1.64723115 & & 1.64723115 & \\
5.0 & 0.88137359 & -9.3 & 0.88137359 & -12.0 \\
& 0.88137359 & & 0.88137359 & \\
10.0 & 0.48121189 & -9.0 & 0.48121189 & -11.4 \\
& 0.48121189 & & 0.48121189 & \\
20.0 & 0.24746646 & -8.7 & 0.24746646 & -11.1 \\
50.0 & 9.24746646 & & 0.24746646 & \\
& $9.98340784 \cdot 10^{-2}$ & -8.3 & $9.98340789 \cdot 10^{-3}$ & -10.6 \\
\hline
\end{tabular}

Tabla 6.3: Para $f(r)=\frac{1-e^{-5 r}}{r} \operatorname{con} J_{0}(r)(\eta=0, \nu=0)$. 
Lo primero que se debe mencionar es la difencia entre el número de puntos necesarios en cada método para realizar el cálculo numérico de la integral. El método de Luna et al. requiere 1250 puntos de evaluación mientras que el método de Wong usa solamente 15 puntos de evaluación para esta prueba comparativa.

Para $p<5$ observamos que el método de Luna et al. funciona bien en la región no asintótica mientras que con el método de Wong no se obtienen buenos resultados.

Para $p \geq 5$ vemos que el método de Luna et al. comienza a fallar y el método de Wong da mejores resultados para la región asintótica hasta $p=50$.

Lo anterior se justifica por el hecho de que el método de Luna et al. no es un método de tipo asintótico por esto es muy efectivo en la región donde los valores de $p$ son pequeños. Por otro lado el método de Wong al ser un método de tipo asintótico es más efectivo en regiones donde los valores de $p$ son grandes.

Con esto se puede pensar en desarrollar un método de tipo híbrido en el que se apliquen ambas metodologías para cada región de $p$ asintótica y no asintótica.

En la Tabla (6.4) se muestra el comparativo para una función con comportamiento Gaussiano $f(r)=e^{-r^{2}}$. La selección de esta función se justifica porque estamos interesados en probar funciones como las que aparecieron en la transformada de Hankel. 


\begin{tabular}{ccccc}
\hline$p$ & $\begin{array}{c}\text { Luna et al. }(\alpha=20) \\
(N \times M)=1250\end{array}$ & $\log _{10}[$ Error $]$ & $\begin{array}{c}\text { Wong } \\
N=15\end{array}$ & $\log _{10}[$ Error $]$ \\
\hline 0.5 & 0.859170309 & -14.6 & NaN & - \\
& 0.859170309 & & 0.859170309 & - \\
1.0 & 0.785150550 & -14.3 & NaN & - \\
& 0.785150550 & & 0.785150550 & \\
5.0 & 0.210416562 & -14.0 & $3.705214007 \cdot 10^{17}$ & - \\
& 0.210416562 & & 0.210416562 & -11.3 \\
10.0 & 0.101049304 & -15.8 & 0.101049304 & -15.0 \\
& 0.101049304 & & 0.101049304 & -14.9 \\
20.0 & $5.012643647 \cdot 10^{-2}$ & -9.9 & $5.012643648 \cdot 10^{-2}$ & \\
& $5.012643648 \cdot 10^{-2}$ & & $5.012643648 \cdot 10^{-2}$ & \\
30.0 & $3.336942420 \cdot 10^{-2}$ & -4.4 & $3.337055729 \cdot 10^{-2}$ & -15.5 \\
& $3.337055729 \cdot 10^{-2}$ & & $3.337055729 \cdot 10^{-2}$ & \\
40.0 & $2.491745719 \cdot 10^{-2}$ & -2.4 & $2.501566918 \cdot 10^{-2}$ & \\
& $2.501566918 \cdot 10^{-2}$ & & $2.501566918 \cdot 10^{-2}$ & \\
50.0 & $1.927353936 \cdot 10^{-2}$ & -1.4 & $2.000801445 \cdot 10^{-3}$ & -15.7 \\
& $2.000801445 \cdot 10^{-2}$ & & $2.000801445 \cdot 10^{-3}$ & \\
\hline
\end{tabular}

Tabla 6.4: Para $f(r)=e^{-r^{2}}$ con $J_{0}(r)(\eta=0, \nu=0)$.

También en este caso el método de Luna et al. requiere 1250 puntos de evaluación y el método de Wong usa 15 puntos de evaluación para realizar la prueba comparativa.

Para $p<5$ observamos que el método de Luna et al. funciona bastante bien en la región no asintótica mientras que el método de Wong falla completamente. En valores de $p$ pequeños el método de Wong presenta problemas con la representación numérica de la máquina $(\mathrm{NaN})$.

Sin embargo para $p>10$ vemos que el método de Luna et al. comienza a fallar y el método de Wong da mejores resultados para la región asintótica hasta $p=50$.

Los resultados mostrados en este comparativo se explican por las características propias de cada uno de los métodos. Esto se debe a que el método de Luna et al. no es un método de tipo asintótico y funciona para valores de $p$ pequeños. Mientras que el método de Wong es un método de tipo asintótico y funciona para valores de $p$ grandes.

También se puede pensar en desarrollar un método de tipo híbrido aplicando ambas metodologías para cada región de $p$. 


\subsection{Conclusiones}

Se definen cuadraturas Gaussianas especializadas basadas en funciones de peso correspondientes a las funciones de Bessel modificadas de tercer tipo $K_{\nu}(r)$ para ser usadas en calcular integrales con funciones que tienen comportamiento exponencial como $f_{1}(r)=e^{-r}$ y $f_{2}(r)=e^{-r^{2}}$.

Las cuadraturas gaussianas para estas funciones de peso se construyen y aplican en el cálculo numérico de integrales con las funciones de Bessel $J_{0}(r)$, $J_{1}(r), J_{2}(r)$ y $J_{3}(r)$. Extendiendo el trabajo original hecho por Wong para la función $J_{0}(r)$.

Los resultados numéricos muestran que en general el método funciona muy bien en las regiones asintóticas con $p$ grande incluso para cuadraturas de orden inferior.

En valores de $p$ cercanos a cero es difícil obtener una mayor precisión aún incrementando el orden de la cuadratura siendo el valor límite $p=0.1$. Por otro lado en el rango de [0.2 - 1.0] es posible obtener resultados precisos con órdenes de cuadratura mayores.

También encontramos casos donde la convergencia es monotóna para las funciones radiales con $m=1, m=2$ y $m=3$. De los cuales no es posible conseguir mayor precisión al aumentar el orden de la cuadratura.

Para las funciones de Bessel de orden superior se deben incluir factores $r^{m}$ en la función de peso asegurando así la convergencia de las integrales en el cálculo de cuadraturas gaussianas referentes al átomo de hidrógeno en dos dimensiones.

El método no funciona para la transformación en el espacio de momentos del oscilador armónico en dos dimensiones.

Se puede aplicar esta metodología para calcular integrales de funciones que poseen expansiones en series multipolares $f(r, \phi)=\sum_{m=-\infty}^{\infty} f_{m}(r) e^{\imath m \phi}$ en donde cada término de la expansión es la transformada de Hankel de $m$-ésimo orden de $f_{m}(r)$. 


\section{Transformada de Bessel esférica}

En la primera parte de este trabajo se planteó la metodología para transformar numéricamente funciones con comportamiento exponencial y Gaussiano del espacio de posición al espacio de momentos. Se usó esta metodología para transformar funciones radiales del átomo de hidrógeno en dos dimensiones. Además se estudió el caso del oscilador armónico en dos dimensiones donde falla el método.

Ahora en esta segunda parte estudiamos el problema en tres dimensiones donde desarrollamos una nueva metodología la cual permite transformar numéricamente funciones de base tipo Slater. También probamos funciones de base tipo Gaussiana ya que para este caso no falla el método con la introducción de un parámetro que llamamos $\alpha$.

La dificultad de resolver este tipo de integrales está en el hecho de que la función $j_{l}(p r)$ en el integrando oscila demasiado para valores de $p$ grandes. Ver la discusión alrededor de la ecuación (4.0.7). Por lo cual es importante encontrar métodos numéricos que permitan calcular con precisión dichas integrales. Algunos autores han trabajado en este problema [60,61] enfatizando en la precisión obtenida [18]. En este sentido estamos interesados en tratar dicho problema mediante una nueva metodología. Ver el planteamiento del método en la sección 4.4. Por lo que desarrollamos el método para resolver el problema en tres dimensiones relacionado con la transformada de Bessel esférica.

El objetivo es examinar el funcionamiento de esta nueva metodología cuando se transforma del espacio de posición al espacio de momentos en 3D. Por lo que probamos funciones con comportamiento exponencial y Gaussiano que aparecen en las funciones de base tipo Slater $f_{n, l}^{S T O}(r)$ y tipo Gaussianas $f_{n, l}^{G T O}(r)$. También exploramos el funcionamiento del método para valores pequeños de la variable $p$. Estudiamos las propiedades de convergencia en varias regiones de $p$. Analizamos su factibilidad para diferentes órdenes $\nu$ de la función de Bessel semientera correspondientes al número cuántico de momento angular $l$. Examinamos el comportamiento del parámetro $\alpha$ que aparece en la transformación de funciones de base tipo Gaussiano. Además comparamos con otra metodología para ver sus alcances.

\subsection{Funciones de base tipo Slater}

Estas funciones con comportamiento exponencial se usan ampliamente como funciones de base en átomos [62] y moléculas [63]. Se pueden representar 
como

$$
f_{n, l}^{S T O}(r)=N_{n, \zeta}^{S T O} r^{n-1} e^{-\zeta r}
$$

$\operatorname{con} \zeta>0, n-1 \geq l \geq 0$ y $N_{n, \zeta}^{S T O}=\left[\frac{(2 \zeta)^{2 n+1}}{\Gamma(2 n+1)}\right]^{\frac{1}{2}}$.

La expresión general para la transformada de Hankel (3.6.6) de estas funciones es

$$
h_{n, l}^{S T O}(p)=N_{n, \zeta}^{S T O} \int_{0}^{\infty}\left[r^{n} e^{-\zeta r}\right] J_{l+\frac{1}{2}}(p r)(p r)^{\frac{1}{2}} \mathrm{~d} r .
$$

En la Tabla (7.1) mostramos las funciones de base tipo Slater normalizadas radiales $\operatorname{con} \zeta=1$ en tres dimensiones [18] que se usaron para probar la metodología propuesta

\begin{tabular}{cccc}
$n$ & $l$ & $f_{n, l}^{S T O}(r)$ & $\tilde{f}_{n, l}^{S T O}(p)$ \\
\hline 1 & 0 & $2 e^{-r}$ & $\frac{4 \sqrt{\frac{2}{\pi}}}{\left(1+p^{2}\right)^{2}}$ \\
2 & 1 & {$\left[\frac{2}{\sqrt{3}}\right] r e^{-r}$} & $\frac{-16 i p \sqrt{\frac{2}{3 \pi}}}{\left(1+p^{2}\right)^{3}}$ \\
3 & 2 & {$\left[\frac{2 \sqrt{\frac{2}{5}}}{3}\right] r^{2} e^{-r}$} & $\frac{-64 p^{2}}{\sqrt{5 \pi}\left(1+p^{2}\right)^{4}}$ \\
4 & 3 & {$\left[\frac{2}{3 \sqrt{35}}\right] r^{3} e^{-r}$} & $\frac{256 i p^{3} \sqrt{\frac{2}{35 \pi}}}{\left(1+p^{2}\right)^{5}}$ \\
5 & 4 & {$\left[\frac{2 \sqrt{\frac{2}{7}}}{45}\right] r^{4} e^{-r}$} & $\frac{1024 p^{4}}{3 \sqrt{7 \pi}\left(1+p^{2}\right)^{6}}$ \\
\hline
\end{tabular}

Tabla 7.1: Funciones de base tipo Slater normalizadas $f_{n, l}^{S T O}(r) \operatorname{con} \zeta=1$ [18]. Las funciones de base tipo Slater en el espacio de momentos son $\tilde{f}_{n, l}^{S T O}(p)$ $[18,58]$.

\subsubsection{Desarrollo}

Es importante resolver numéricamente integrales que tienen funciones de Bessel esféricas $j_{l}(p r)$ por ser usadas como eigenfunciones para sistemas de coordenadas esféricas [64]

En química cuántica aparecen las funciones tipo Slater que se utilizan como base para los orbitales atómicos por lo que se representa la parte radial como en (7.1.1) siendo la función transformada

$$
I(l, p)=\int_{0}^{\infty} r^{2} f(r) j_{l}(p r) \mathrm{d} r
$$


donde $f(r)=f_{n, l}^{S T O}(r)$ y $l$ es el número cuántico de momento angular.

El problema radica en la evaluación numérica de la ecuación (7.1.3) para funciones que decaen lentamente en valores grandes de $p$ cuando el integrando oscila demasiado.

Wong [36] introdujo una metodología para tratar a las funciones de Bessel de orden entero $J_{m}(p r)$. Por lo que en esta parte desarrollamos una nueva metodología para funciones de Bessel esféricas $j_{l}(p r)$.

Para la función de base tipo Slater radial $f_{1,0}^{S T O}(r)(l=0)$ sustituimos $j_{0}(p r)$ usando la relación de la función de Bessel de orden semientero con la función de Bessel esférica

$$
j_{l}(p r)=\sqrt{\frac{\pi}{2 r}} J_{\frac{1}{2}+l}(p r),
$$

por lo cual $j_{0}(p r)=\sqrt{\frac{\pi}{2 r}} J_{\frac{1}{2}}(p r)$ que al sustituirse en la ecuación (7.1.3)

$$
I(l, p)=\frac{\pi^{1 / 2}}{2^{1 / 2} p^{1 / 2}} \int_{0}^{\infty} f(r) J_{\frac{1}{2}}(p r) r^{3 / 2} \mathrm{~d} r,
$$

se cumple la condición $(2+l) \pm\left(\frac{1}{2}+l\right)>-1$ a fin de asegurar la convergencia de las integrales [36].

Se transforma a las funciones de Bessel modificadas de segundo tipo $K_{\frac{1}{2}}(t)$ de donde sabemos que se cumple $J_{\frac{1}{2}}(t)=\frac{1}{2}\left\{H_{\frac{1}{2}}^{(1)}(t)+H_{\frac{1}{2}}^{(2)}(t)\right\}$ además de $H_{\frac{1}{2}}^{(1)}(t)=\frac{2}{\pi \imath} e^{-\imath \pi \nu / 2} K_{\frac{1}{2}}(-\imath t)$ y $H_{\frac{1}{2}}^{(2)}(t)=-\frac{2}{\pi \imath} e^{\imath \pi \nu / 2} K_{\frac{1}{2}}(\imath t)$. Al sustituirse en (7.1.5) y realizar los correspondientes cambios de variable tenemos

$$
\begin{array}{r}
I(l, p)=\left(\frac{\pi^{1 / 2}}{2^{1 / 2} p^{1 / 2}}\right)\left(\frac{1}{\pi p^{5 / 2}}\right)\left[e^{\imath \pi / 2} \int_{0}^{\infty} f(\imath r / p) K_{\frac{1}{2}}(r) r^{3 / 2} \mathrm{~d} r+\right. \\
\left.e^{-\imath \pi / 2} \int_{0}^{\infty} f(-\imath r / p) K_{\frac{1}{2}}(r) r^{3 / 2} \mathrm{~d} r\right]
\end{array}
$$

Considerando que la función de Bessel esférica modificada de tercer tipo $K_{l+\frac{1}{2}}(r)$ se puede escribir en términos de funciones elementales a través de la siguiente relación de recurrencia $[37,38]$

$$
f_{l}(r)=(-1)^{l+1} \sqrt{\frac{\pi}{2 r}} K_{l+\frac{1}{2}}(r) \quad l=0,1,2, \ldots,
$$

donde sabemos que

$$
K_{-l-\frac{1}{2}}(r)=K_{l+\frac{1}{2}}(r),
$$




$$
f_{l-1}(r)-f_{l+1}(r)=\frac{2 l+1}{r} f_{l}(r),
$$

para $l=0$ usamos $K_{\frac{1}{2}}(r)=\sqrt{\frac{\pi}{2 r}} e^{-r}[38]$ en la ecuación (7.1.6)

$$
\begin{array}{r}
I(l, p)=\left(\frac{1}{2^{1 / 2} \pi^{1 / 2} p^{3}}\right)\left(\frac{\pi^{1 / 2}}{2^{1 / 2}}\right)\left[\imath \int_{0}^{\infty} f(\imath r / p) r e^{-r} \mathrm{~d} r-\right. \\
\left.\iota \int_{0}^{\infty} f(-\imath r / p) r e^{-r} \mathrm{~d} r\right],
\end{array}
$$

separamos a la función de peso para obtener

$$
\begin{gathered}
I(l, p)=\frac{\imath}{2 p^{3}}\left[\int_{0}^{\infty} r f(\imath r / p) e^{-r} \mathrm{~d} r-\int_{0}^{\infty} r f(-\imath r / p) e^{-r} \mathrm{~d} r\right], \\
I(l, p)=\frac{1}{2 p^{2}}\left[\int_{0}^{\infty}(\imath r / p) f(\imath r / p) e^{-r} \mathrm{~d} r+\int_{0}^{\infty}(-\imath r / p) f(-\imath r / p) e^{-r} \mathrm{~d} r\right],
\end{gathered}
$$

en donde dicha función de peso es $w(r)=e^{-r}$. Se realizó el mismo procedimiento para funciones de base tipo Slater radiales $l=1, l=2, l=3$ y $l=4$.

También es importante notar de las Ecs. (7.1.7 - 7.1.9) que las funciones de Bessel esféricas modificadas de orden semientero $K_{l+\frac{1}{2}}$ pueden ser representadas en términos de funciones exponenciales lo cual no se puede hacer para el caso de las funciones de Bessel de orden entero $K_{m}$.

Ahora veamos que si se emplea la definición de las funciones de Bessel esféricas en términos de funciones trigonométricas es posible obtener la misma representación (7.1.12) sin usar el procedimiento anterior para calcular la transformada de Bessel esférica.

Por lo que comenzamos definiendo a la función de Bessel esférica para $l=0$

$$
j_{0}(p r)=\frac{\operatorname{sen} p r}{p r}
$$

que al sustituirse en la ecuación (7.1.3)

$$
I(l, p)=\int_{0}^{\infty} r^{2} f(r)\left[\frac{\operatorname{sen} p r}{p r}\right] \mathrm{d} r,
$$




$$
I(l, p)=\frac{1}{p} \int_{0}^{\infty} r f(r) \operatorname{sen} p r d r
$$

representando la expresión en términos de exponenciales tenemos que

$$
\begin{gathered}
I(l, p)=\frac{1}{p} \int_{0}^{\infty} r f(r)\left(\frac{e^{\imath p r}-e^{-\imath p r}}{2 \imath}\right) \mathrm{d} r, \\
I(l, p)=\frac{1}{2 \imath p} \int_{0}^{\infty} r f(r)\left(e^{\imath p r}-e^{-\imath p r}\right) \mathrm{d} r \\
I(l, p)=\frac{1}{2 \imath p}\left[\int_{0}^{\infty} r f(r) e^{\imath p r} \mathrm{~d} r-\int_{0}^{\infty} r f(r) e^{-\imath p r} \mathrm{~d} r\right] .
\end{gathered}
$$

Efectuamos los siguientes cambios de variable $z= \pm \imath p r$, despejando a $r$ tenemos que $r= \pm \imath z / p$ y sustituyendo en la ecuación anterior

$$
\begin{array}{r}
I(l, p)=\frac{1}{2 \imath p}\left[\int_{0}^{\infty}(\imath z / p) f(\imath z / p) e^{-z}(\imath \mathrm{d} z / p)-\right. \\
\left.\int_{0}^{\infty}(-\imath z / p) f(-\imath z / p) e^{-z}(-\imath \mathrm{d} z / p)\right]
\end{array}
$$

finalmente llegamos a la misma representación obtenida en (7.1.12).

$$
I(l, p)=\frac{1}{2 p^{2}}\left[\int_{0}^{\infty}(\imath z / p) f(\imath z / p) e^{-z} \mathrm{~d} z+\int_{0}^{\infty}(-\imath z / p) f(-\imath z / p) e^{-z} \mathrm{~d} z\right],
$$

con función de peso $w(z)=e^{-z}$.

Se realizó el mismo procedimiento para funciones de base tipo Slater radiales $l=1, l=2, l=3$ y $l=4$.

Es importante resaltar que las funciones de peso $w(r)$ que aparecen para esta aplicación de la transformada de Bessel esférica están relacionadas con los polinomios de Bessel inversos $\Theta_{l}(r)$. Esto se debe a que pueden escribirse las funciones de Bessel esféricas modificadas de tercer $K_{l+\frac{1}{2}}(r)$ tipo como [65]

$$
K_{l+\frac{1}{2}}(r)=\sqrt{\frac{\pi}{2}} r^{-\left(l+\frac{1}{2}\right)} e^{-r} \Theta_{l}(r) .
$$


Por lo cual presentamos en la Tabla (7.2) los primeros cinco polinomios de Bessel inversos $\Theta_{l}(r)$ asociados a las funciones de peso $w_{n, l}^{S T O}(r)$

\begin{tabular}{lc}
$l$ & $\Theta_{l}(r)$ \\
\hline 0 & 1 \\
1 & $r+1$ \\
2 & $r^{2}+3 r+3$ \\
3 & $r^{3}+6 r^{2}+15 r+15$ \\
4 & $r^{4}+10 r^{3}+45 r^{2}+105 r+105$ \\
\hline
\end{tabular}

Tabla 7.2: Los polinomios de Bessel inversos $\Theta_{l}(r)$ para $0 \leq l \leq 4$.

Es posible generalizar este procedimiento para calcular las transformadas de Bessel esféricas de funciones de base tipo Slater radiales así la ecuación (4.4.9) toma la forma

$$
\begin{array}{r}
\tilde{f}_{n, l}(p) \approx \frac{\imath^{l}}{2 p^{l+2}}\left\{\sum_{k=1}^{N}\left(\imath r_{k} / p\right) f_{n, l}\left(\imath r_{k} / p\right) w_{k}+\right. \\
\left.\sum_{k=1}^{N}\left(-\imath r_{k} / p\right) f_{n, l}\left(-\imath r_{k} / p\right) w_{k}\right\} .
\end{array}
$$

Donde las $r_{k}$ y $w_{k}$ son las abscisas y pesos de la cuadratura Gaussiana con función de peso $w_{n, l}^{S T O}(r)$. El factor $\frac{ \pm \imath r_{k}}{p}$ representa la factorización del elemento de volumen $r^{2} \mathrm{~d} r$ en este procedimiento. Se enfatiza que $f_{n, l}(r)=r^{n-(l+1)} e^{-\zeta r}$ es distinto a la definición de la función de base tipo Slater $\left(r^{n-1} e^{-\zeta r}\right)$, esto se debe a que el factor $r^{l}$ está incluido en la función de peso para poder obtener los polinomios de Bessel inversos $\Theta_{l}(r)$.

Las soluciones en forma cerrada de las integrales (7.1.3) están disponibles en la Tabla (7.1). Las representaciones analíticas de las funciones en el espacio de momentos pueden usarse para probar el procedimiento numérico y obtener las transformaciones de las funciones del espacio de posición.

\subsubsection{Procedimiento}

Polinomios ortogonales y fórmulas de cuadraturas Gaussianas para funciones de peso $w_{n, l}^{S T O}(r)$ en el intervalo $[0, \infty]$ se obtuvieron hasta el orden $N=145$ utilizando el procedimiento de Stieltjes discretizado, ver apéndice C (12). 


\begin{tabular}{cc}
$l$ & $w_{n, l}^{S T O}(r)$ \\
\hline 0 & $e^{-r} \Theta_{0}(r)$ \\
1 & $e^{-r} \Theta_{1}(r)$ \\
2 & $e^{-r} \Theta_{2}(r)$ \\
3 & $e^{-r} \Theta_{3}(r)$ \\
4 & $e^{-r} \Theta_{4}(r)$ \\
\hline
\end{tabular}

Tabla 7.3: Funciones de peso $w_{n, l}^{S T O}(r)$ dependientes de los polinomios de Bessel inversos $\Theta_{l}(r)$ ver Tabla (7.2).

Se realizó un análisis de los resultados numéricos obtenidos utilizando este método para cinco funciones de base tipo STO (Slater-type orbital) correspondientes a $l=0, l=1, l=2, l=3$ y $l=4$ que fueron comparados con los valores analíticos de la Tabla (7.1) para un valor de $\zeta=1$ en (7.1.1). Los resultados obtenidos se analizan y presentan en gráficas que aparecen en la sección (7.4).

\subsection{Funciones de base tipo Gaussianas}

Las funciones de tipo Gaussiano se definen como la clase de funciones con comportamiento de largo alcance dominante en una exponencial Gaussiana [18]. Fueron introducidas en cálculos atómicos y moleculares en 1950 [66,67] y posteriormente las funciones de base para orbitales tipo Gaussiano [68]. Tienen la siguiente forma

$$
f_{n, l}^{G T O}(r)=N_{n, \zeta}^{G T O} r^{n-1} e^{-\zeta r^{2}}
$$

donde $\zeta>0, n-1 \geq l \geq 0$ y $N_{n, \zeta}^{G T O}=\left[\frac{2(2 \zeta)^{n+\frac{1}{2}}}{\Gamma\left(n+\frac{1}{2}\right)}\right]^{\frac{1}{2}}$.

La transformada de Hankel (3.6.6) para estas funciones es

$$
h_{n, l}^{G T O}(p)=N_{n, \zeta}^{G T O} \int_{0}^{\infty}\left[r^{n} e^{-\zeta r^{2}}\right] J_{l+\frac{1}{2}}(p r)(p r)^{\frac{1}{2}} \mathrm{~d} r .
$$

En la Tabla (7.4) mostramos las funciones de base tipo Gaussianas normalizadas radiales con $\zeta=5$ en tres dimensiones [18] que se usaron para probar la metodología propuesta

\subsubsection{Desarrollo}

Veamos el procedimiento para obtener la transformación de una función de base tipo Gaussiana con $l=0$. 


\begin{tabular}{cccc}
$n$ & $l$ & $f_{n, l}^{G T O}(r)$ & $\widetilde{f}_{n, l}^{G T O}(p)$ \\
\hline 1 & 0 & {$\left[2 \sqrt[4]{\frac{10^{3}}{\pi}}\right] e^{-5 r^{2}}$} & $\sqrt[4]{\frac{2}{5^{3} \pi}} e^{-\frac{p^{2}}{20}}$ \\
2 & 1 & {$\left[\frac{20 \sqrt[4]{\frac{2^{3} \cdot 5}{\pi}}}{\sqrt{3}}\right] r e^{-5 r^{2}}$} & $\frac{-\imath p \sqrt[4]{\frac{2}{5 \pi}} e^{-\frac{p^{2}}{20}}}{5 \sqrt{3}}$ \\
3 & 2 & {$\left[\frac{40 \sqrt[4]{\frac{2^{3} \cdot 5}{\pi}}}{\sqrt{3}}\right] r^{2} e^{-5 r^{2}}$} & $\frac{-p^{2} \sqrt[4]{\frac{2}{5 \pi}} e^{-\frac{p^{2}}{20}}}{25 \sqrt{3}}$ \\
4 & 3 & {$\left[\frac{80 \sqrt[4]{\frac{10^{3}}{\pi}}}{\sqrt{21}}\right] r^{3} e^{-5 r^{2}}$} & $\frac{\imath p^{3} \sqrt[4]{\frac{2}{5^{3} \pi}} e^{-\frac{p^{2}}{20}}}{25 \sqrt{21}}$ \\
\hline
\end{tabular}

Tabla 7.4: Funciones de base tipo Gaussianas normalizadas $f_{n, l}^{G T O}(r)$ con $\zeta=5$ [18]. Las funciones de base tipo Gaussianas en el espacio de momentos son $\widetilde{f}_{n, l}^{G T O}(p)[18,58]$.

Utilizando la representación de la transformada de Bessel esférica (7.1.3) tal y como se hizo con la función de base tipo Slater $l=0$ llegamos a la representación

$$
I(l, p)=\frac{\pi^{1 / 2}}{2^{1 / 2} p^{1 / 2}} \int_{0}^{\infty} f(r) J_{\frac{1}{2}}(p r) r^{3 / 2} \mathrm{~d} r
$$

que cumple la condición de $(2+l) \pm\left(\frac{1}{2}+l\right)>-1$. Se realizan algunas transformaciones hasta llegar a las funciones de Bessel esféricas modificadas de tercer tipo $K_{\frac{1}{2}}(r)$

$$
\begin{gathered}
I(l, p)=\left(\frac{\pi^{1 / 2}}{2^{1 / 2} p^{1 / 2}}\right)\left(\frac{1}{\pi p^{5 / 2}}\right)\left[e^{\imath \pi / 2} \int_{0}^{\infty} f(\imath r / p) K_{\frac{1}{2}}(r) r^{3 / 2} \mathrm{~d} r+\right. \\
\left.e^{-\imath \pi / 2} \int_{0}^{\infty} f(-\imath r / p) K_{\frac{1}{2}}(r) r^{3 / 2} \mathrm{~d} r\right], \quad(7.2 .4) \\
I(l, p)=\left(\frac{1}{2^{1 / 2} \pi^{1 / 2} p^{3}}\right)\left(\frac{\pi^{1 / 2}}{2^{1 / 2}}\right)\left[\imath \int_{0}^{\infty} f(\imath r / p) r e^{-r} \mathrm{~d} r-\imath \int_{0}^{\infty} f(-\imath r / p) r e^{-r} \mathrm{~d} r\right], \\
I(l, p)=\frac{\imath}{2 p^{3}}\left[\int_{0}^{\infty} r f(\imath r / p) e^{-r} \mathrm{~d} r-\int_{0}^{\infty} r f(-\imath r / p) e^{-r} \mathrm{~d} r\right],
\end{gathered}
$$




$$
I(l, p)=\frac{1}{2 p^{2}}\left[\int_{0}^{\infty}(\imath r / p) f(\imath r / p) e^{-r} \mathrm{~d} r+\int_{0}^{\infty}(-\imath r / p) f(-\imath r / p) e^{-r} \mathrm{~d} r\right]
$$

En este punto y por el carácter par de la función $f(r)=f_{n, l}^{G T O}(r)$ ambas integrales en (7.2.7) son iguales en magnitud y opuestas en signo por lo que se cancelan como en el caso 2D en la transformada de Hankel para el oscilador armónico.

Sin embargo al introducir el factor $e^{\imath \alpha r / p} e^{-\imath \alpha r / p}$ en la expresión de arriba se puede romper la simetría evitando la cancelación. Lo anterior sirve porque las funciones de Bessel esféricas de tercer tipo pueden escribirse en términos de funciones elementales como las exponenciales [37]. Sustituyendo este factor se obtiene

$$
\begin{gathered}
I(l, p)=\frac{1}{2 p^{2}}\left[\int_{0}^{\infty}(\imath r / p) f(\imath r / p) e^{\imath \alpha r / p} e^{-\imath \alpha r / p} e^{-r} \mathrm{~d} r+\right. \\
\left.\int_{0}^{\infty}(-\imath r / p) f(-\imath r / p) e^{\imath \alpha r / p} e^{-\imath \alpha r / p} e^{-r} \mathrm{~d} r\right], \\
I(l, p)=\frac{1}{2 p^{2}}\left[\int_{0}^{\infty}(\imath r / p) f(\imath r / p) e^{\imath \alpha r / p} e^{-r\left(1+\frac{\imath \alpha}{p}\right)} \mathrm{d} r+\right. \\
\left.\int_{0}^{\infty}(-\imath r / p) f(-\imath r / p) e^{-\imath \alpha r / p} e^{-r\left(1-\frac{\imath \alpha}{p}\right)} \mathrm{d} r\right] .
\end{gathered}
$$

Efectuamos los siguientes cambios de variable $z=r(1 \pm \imath \alpha / p)$ despejando a $r$ tenemos $r=z /(1 \pm \imath \alpha / p) \equiv z p /(p \pm \imath \alpha)$ que sustituyendo en la ecuación anterior

$$
\begin{array}{r}
I(l, p)=\frac{1}{2 p^{2}}\left[\int_{0}^{\infty}\left(\frac{\imath z}{p+\imath \alpha}\right) f\left(\frac{\imath z}{p+\imath \alpha}\right) e^{\left(\frac{\imath \alpha z}{p+\imath \alpha}\right)} \frac{p e^{-z}}{(p+\imath \alpha)} \mathrm{d} z+\right. \\
\left.\int_{0}^{\infty}\left(\frac{-\imath z}{p-\imath \alpha}\right) f\left(\frac{-\imath z}{p-\imath \alpha}\right) e^{\left(\frac{-\imath \alpha z}{p-\imath \alpha}\right)} \frac{p e^{-z}}{(p-\imath \alpha)} \mathrm{d} z\right], \\
I(l, p)=\frac{1}{2 p^{2}}\left[\frac{p}{(p+\imath \alpha)} \int_{0}^{\infty}\left(\frac{\imath z}{p+\imath \alpha}\right) f\left(\frac{\imath z}{p+\imath \alpha}\right) e^{\left(\frac{\imath \alpha z}{p+\imath \alpha}\right)} e^{-z} \mathrm{~d} z+\right. \\
\left.\frac{p}{(p-\imath \alpha)} \int_{0}^{\infty}\left(\frac{-\imath z}{p-\imath \alpha}\right) f\left(\frac{-\imath z}{p-\imath \alpha}\right) e^{\left(\frac{-\imath \alpha z}{p-\imath \alpha}\right)} e^{-z} \mathrm{~d} z\right],
\end{array}
$$




$$
\begin{array}{r}
I(l, p)=\frac{1}{2 p}\left[\frac{1}{(p+\imath \alpha)} \int_{0}^{\infty}\left(\frac{\imath z}{p+\imath \alpha}\right) f\left(\frac{\imath z}{p+\imath \alpha}\right) e^{\left(\frac{\imath \alpha z}{p+\imath \alpha}\right)} e^{-z} \mathrm{~d} z+\right. \\
\left.\frac{1}{(p-\imath \alpha)} \int_{0}^{\infty}\left(\frac{-\imath z}{p-\imath \alpha}\right) f\left(\frac{-\imath z}{p-\imath \alpha}\right) e^{\left(\frac{-\imath \alpha z}{p-\imath \alpha}\right)} e^{-z} \mathrm{~d} z\right],
\end{array}
$$

esta es la expresión usada para realizar cálculos en una función de base tipo Gaussiana radial con $l=0$ y función de peso $w(z)=e^{-z}$.

Ahora vamos a obtener la expresión (7.2.12) a partir de la definición de las funciones de Bessel esféricas en términos de funciones trigonométricas usando la definición (7.1.13) que al sustituirse en la ecuación (7.1.3) se tiene

$$
\begin{gathered}
I(l, p)=\int_{0}^{\infty} r^{2} f(r)\left[\frac{\operatorname{sen} p r}{p r}\right] \mathrm{d} r \\
I(l, p)=\frac{1}{p} \int_{0}^{\infty} r f(r) \text { sen } p r \mathrm{~d} r
\end{gathered}
$$

representamos la expresión trigonométrica en términos de exponenciales

$$
\begin{gathered}
I(l, p)=\frac{1}{p} \int_{0}^{\infty} r f(r)\left(\frac{e^{\imath p r}-e^{-\imath p r}}{2 \imath}\right) \mathrm{d} r \\
I(l, p)=\frac{1}{2 \imath p} \int_{0}^{\infty} r f(r)\left(e^{\imath p r}-e^{-\imath p r}\right) \mathrm{d} r \\
I(l, p)=\frac{1}{2 \imath p}\left[\int_{0}^{\infty} r f(r) e^{\imath p r} \mathrm{~d} r-\int_{0}^{\infty} r f(r) e^{-\imath p r} \mathrm{~d} r\right] .
\end{gathered}
$$

Debido a que la expresión de arriba da cero por la paridad de la función Gaussiana $f(r)$ introducimos el factor $e^{\alpha r} e^{-\alpha r}$ para evitar la cancelación de términos

$$
I(l, p)=\frac{1}{2 \imath p}\left[\int_{0}^{\infty} r f(r) e^{\alpha r} e^{-\alpha r} e^{\imath p r} \mathrm{~d} r-\int_{0}^{\infty} r f(r) e^{\alpha r} e^{-\alpha r} e^{-\imath p r} \mathrm{~d} r\right],
$$




$$
I(l, p)=\frac{1}{2 \imath p}\left[\int_{0}^{\infty} r f(r) e^{\alpha r} e^{-r(\alpha-\imath p)} \mathrm{d} r-\int_{0}^{\infty} r f(r) e^{\alpha r} e^{-r(\alpha+\imath p)} \mathrm{d} r\right]
$$

Realizamos los siguientes cambios de variable $z=r(\alpha \mp \imath p)$ despejando a $r$ tenemos que $r=z /(\alpha \mp \imath p)$ y sustituyendo en la ecuación anterior

$$
\begin{aligned}
I(l, p)=\frac{1}{2 \imath p}\left[\int_{0}^{\infty}\left(\frac{z}{\alpha-\imath p}\right) f\left(\frac{z}{\alpha-\imath p}\right) e^{\left(\frac{\alpha z}{\alpha-\imath p}\right)} \frac{e^{-z}}{(\alpha-\imath p)} \mathrm{d} z-\right. \\
\int_{0}^{\infty}\left(\frac{z}{\alpha+\imath p}\right) f\left(\frac{z}{\alpha+\imath p}\right) e^{\left.\left(\frac{\alpha z}{\alpha+\imath p}\right) \frac{e^{-z}}{(\alpha+\imath p)} \mathrm{d} z\right],} \\
I(l, p)=\frac{1}{2 \imath p}\left[\frac{1}{(\alpha-\imath p)} \int_{0}^{\infty}\left(\frac{z}{\alpha-\imath p}\right) f\left(\frac{z}{\alpha-\imath p}\right) e^{\left(\frac{\alpha z}{\alpha-\imath p}\right)} e^{-z} \mathrm{~d} z-\right. \\
\left.\frac{1}{(\alpha+\imath p)} \int_{0}^{\infty}\left(\frac{z}{\alpha+\imath p}\right) f\left(\frac{z}{\alpha+\imath p}\right) e^{\left(\frac{\alpha z}{\alpha+\imath p}\right)} e^{-z} \mathrm{~d} z\right] .
\end{aligned}
$$

Multiplicamos por el factor $(\imath / \imath)$ los argumentos de la primera integral y por el factor $(-\imath /-\imath)$ los argumentos de la segunda integral

$$
\begin{array}{r}
I(l, p)=\frac{1}{2 p}\left[\frac{1}{(p+\imath \alpha)} \int_{0}^{\infty}\left(\frac{\imath z}{p+\imath \alpha}\right) f\left(\frac{\imath z}{p+\imath \alpha}\right) e^{\left(\frac{\imath \alpha z}{p+\imath \alpha}\right)} e^{-z} \mathrm{~d} z-\right. \\
\left.\frac{1}{(-p+\imath \alpha)} \int_{0}^{\infty}\left(\frac{-\imath z}{p-\imath \alpha}\right) f\left(\frac{-\imath z}{p-\imath \alpha}\right) e^{\left(\frac{-\imath \alpha z}{p-\imath \alpha}\right)} e^{-z} \mathrm{~d} z\right],
\end{array}
$$

para finalmente llegar a la misma representación que en (7.2.12)

$$
\begin{array}{r}
I(l, p)=\frac{1}{2 p}\left[\frac{1}{(p+\imath \alpha)} \int_{0}^{\infty}\left(\frac{\imath z}{p+\imath \alpha}\right) f\left(\frac{\imath z}{p+\imath \alpha}\right) e^{\left(\frac{\imath \alpha z}{p+\imath \alpha}\right)} e^{-z} \mathrm{~d} z+\right. \\
\left.\frac{1}{(p-\imath \alpha)} \int_{0}^{\infty}\left(\frac{-\imath z}{p-\imath \alpha}\right) f\left(\frac{-\imath z}{p-\imath \alpha}\right) e^{\left(\frac{-\imath \alpha z}{p-\imath \alpha}\right)} e^{-z} \mathrm{~d} z\right] .
\end{array}
$$

Se realizó el mismo procedimiento para funciones de base tipo Gaussianas radiales $l=1, l=2$ y $l=3$.

En las transformaciones de funciones de base tipo Gaussianas para cada $l$ se requieren $l+1$ funciones de peso $w_{n, l}^{G T O}(r)$ ver Tabla (7.5). Lo anterior 
se debe a la introducción del factor que rompe la simetría que provoca la partición de la ecuación (4.4.9) en varias sumas.

Es posible generalizar este procedimiento para calcular las transformadas de Bessel esféricas de funciones de base tipo Gaussianas radiales; así, la ecuación (4.4.9) toma la forma

$$
\begin{aligned}
\widetilde{f}_{n, l}^{(j)}(p) \approx \frac{\imath^{l}}{2 p^{l+1}}\left\{\sum_{k=1}^{N}\right. & \left(\frac{\imath z_{k}}{p+\imath \alpha}\right) f_{n, l}\left(\frac{\imath z_{k}}{p+\imath \alpha}\right) e^{\left(\frac{\imath \alpha z_{k}}{p+\imath \alpha}\right)} w_{k}^{(j)}+ \\
& \left.\sum_{k=1}^{N}\left(\frac{-\imath z_{k}}{p-\imath \alpha}\right) f_{n, l}\left(\frac{-\imath z_{k}}{p-\imath \alpha}\right) e^{\left(\frac{-\imath \alpha z_{k}}{p-\imath \alpha}\right)} w_{k}^{(j)}\right\} .
\end{aligned}
$$

Donde las $z_{k}$ y $w_{k}$ son las abscisas y pesos de la cuadratura Gaussiana con función de peso $w_{n, l}^{G T O}(r)$. El factor $\frac{ \pm \imath z_{k}}{p \pm \imath \alpha}$ representa la factorización del elemento de volumen $r^{2} \mathrm{~d} r$ en este procedimiento. Es importante destacar que $f_{n, l}(r)=r^{n-(l+1)} e^{-\zeta r^{2}}$ se considera que es distitnto a la definición de la función de base tipo Gaussiana $\left(r^{n-1} e^{-\zeta r^{2}}\right)$ esto por el hecho de que el factor $r^{l}$ está incluido en la función de peso para poder obtener los polinomios de Bessel inversos $\Theta_{l}(r)$ y los polinomios $P_{n}^{(l-1)}(-r)$ correspondientes. Por lo que la aproximación total $\widetilde{f}_{n, l}(p)$ está dada por la suma de cada una de las particiones referentes a las funciones de peso $w^{(j)}$

$$
\widetilde{f}_{n, l}(p) \approx \sum_{j=1}^{l+1} M_{l}^{(j)} \widetilde{f}_{n, l}^{(j)}(p)
$$

siendo el factor $M_{l}^{(j)}$

$$
M_{l}^{(j)}=\kappa^{(j)}( \pm \imath \alpha)^{j-1} p^{l-j+1},
$$

con los $\kappa^{(j)}$ siendo los coeficientes constantes del polinomio de Bessel inverso correspondiente a $l$ considerando el valor positivo del término $( \pm \imath \alpha)^{j-1}$ para la primera suma y el negativo para la segunda suma en (7.2.24).

Las soluciones en forma cerrada de estas integrales están disponibles en la Tabla (7.4). Las representaciones analíticas de las funciones en el espacio de momentos pueden usarse para probar el procedimiento numérico y obtener las transformaciones de las funciones del espacio de posición.

\subsubsection{Procedimiento}

Polinomios ortogonales y fórmulas de cuadraturas Gaussianas para funciones de peso $w_{n, l}^{G T O}(r)$ en el intervalo $[0, \infty]$ se obtuvieron hasta el orden $N=145$ utilizando el procedimiento de Stieltjes discretizado. 


\begin{tabular}{cc}
$l$ & $w_{n, l}^{G T O}(r)$ \\
\hline 0 & $e^{-r} \Theta_{0}(r)$ \\
1 & $e^{-r} \Theta_{0}(r)$ \\
& $e^{-r} \Theta_{1}(r)$ \\
2 & $e^{-r} \Theta_{0}(r)$ \\
& $e^{-r}(r+2)$ \\
& $e^{-r} \Theta_{2}(r)$ \\
3 & $e^{-r} \Theta_{0}(r)$ \\
& $e^{-r}(r+3)$ \\
& $e^{-r}\left(2 r^{2}+10 r+15\right)$ \\
& $e^{-r} \Theta_{3}(r)$ \\
\hline
\end{tabular}

Tabla 7.5: Funciones de peso $w_{n, l}^{G T O}(r)$ dependientes de los polinomios de Bessel inversos $\Theta_{l}(r)$ y de los polinomios $P_{n}^{(l-1)}(-r)$ para $l \geq 0$.

De la tabla anterior se observa que $\Theta_{1}(r) \equiv P_{1}^{(0)}(-r)$ además tenemos $P_{1}^{(1)}(-r)=(r+2)$ y $P_{1}^{(2)}(-r)=(r+3)$.

Se realizó un análisis de los resultados numéricos obtenidos utilizando este método para cuatro funciones de base tipo GTO (Gaussian-type orbital) correspondientes a $l=0, l=1, l=2$ y $l=3$ que fueron comparados con los valores analíticos de la Tabla (7.4) para un valor de $\zeta=5$ en (7.2.1) y parámetro $\alpha=20$ en (7.2.11). Los resultados obtenidos se analizan y presentan en gráficas que aparecen en la sección (7.4).

\subsubsection{Análisis del parámetro $\alpha$}

Es importante analizar el parámetro $\alpha$ ya que la selección adecuada de un valor nos permite obtener resultados satisfactorios en el cálculo numérico de las transformadas de Bessel esféricas aplicadas a funciones de base tipo Gaussianas.

El parámetro $\alpha$ lleva a la transformación $r=z p /(p \pm \imath \alpha)$ que da como resultado la evaluación de argumentos con la forma $\frac{ \pm \imath z}{p \pm \imath \alpha}$ ver ecuaciones (7.2.8-7.2.11), donde tenemos que el argumento a evaluar está en función de tres términos $(\alpha, p, z)$. Los nodos $x_{k}$ de las cuadraturas calculadas corresponden a la discretización de la variable $z$.

El estudio del comportamiento del parámetro se hizo para dos regiones de $\alpha$ graficando el valor de $z$ (nodos de la cuadratura) contra el valor de la parte real de $\left(\frac{\imath z}{p+\imath \alpha}\right) f_{n, l}\left(\frac{\imath z}{p+\imath \alpha}\right) e^{\left(\frac{\imath \alpha z}{p+\imath \alpha}\right)}$ que aparece en la ecuación (7.2.24), por lo que se escogió la función de prueba $R_{1,0}$ con $f_{1,0}(x)=e^{-x^{2}}$ 


$$
\operatorname{Re}\left[R_{1,0}\right]=\left(\frac{\imath z}{p+\imath \alpha}\right) f_{1,0}\left(\frac{\imath z}{p+\imath \alpha}\right) e^{\left(\frac{\imath \alpha z}{p+\imath \alpha}\right)},
$$

en este caso tomamos el argumento con signo positivo ya que para el argumento con signo negativo obtenemos el mismo resultado.

\subsubsection{1 $\alpha<1$}

Cuando $\alpha=0$ el argumento $\frac{ \pm \imath z}{p \pm \imath \alpha}$ se convierte en $\frac{ \pm \imath z}{p}$ que aparece en las funciones de base tipo Slater que no tienen el parámetro $\alpha$. Por lo que para valores de $\alpha$ cercanos a cero el comportamiento del método se aproxima al caso en el que no existe parámetro $\alpha$.

El argumento $\frac{ \pm \imath z}{p}$ se acota en valores de $p$ grandes, es decir, tiende a valores pequeños del argumento lo cual le da el carácter asintótico a la metodología para funciones de base tipo Slater. Sin embargo con la introducción del parámetro $\alpha$ a través de las funciones exponenciales el comportamiento del método cambia dejando de ser asintótico.

- Caso I. Fig. (7.1). Para $0<\alpha<1$ y $0<p<1$. Observamos que para $\alpha=0.1$ (cercano a cero) el valor de Re $\left[R_{1,0}\right]$ oscila o crece más que para $\alpha=0.25$ y $\alpha=0.5$ independientemente de los valores de $p$ en el intervalo $(0,1)$. Esto demuestra que $\alpha$ amortigua la oscilación de la función. Además $\alpha<1$ se aproxima al comportamiento de la función sin la introducción del parámetro. 

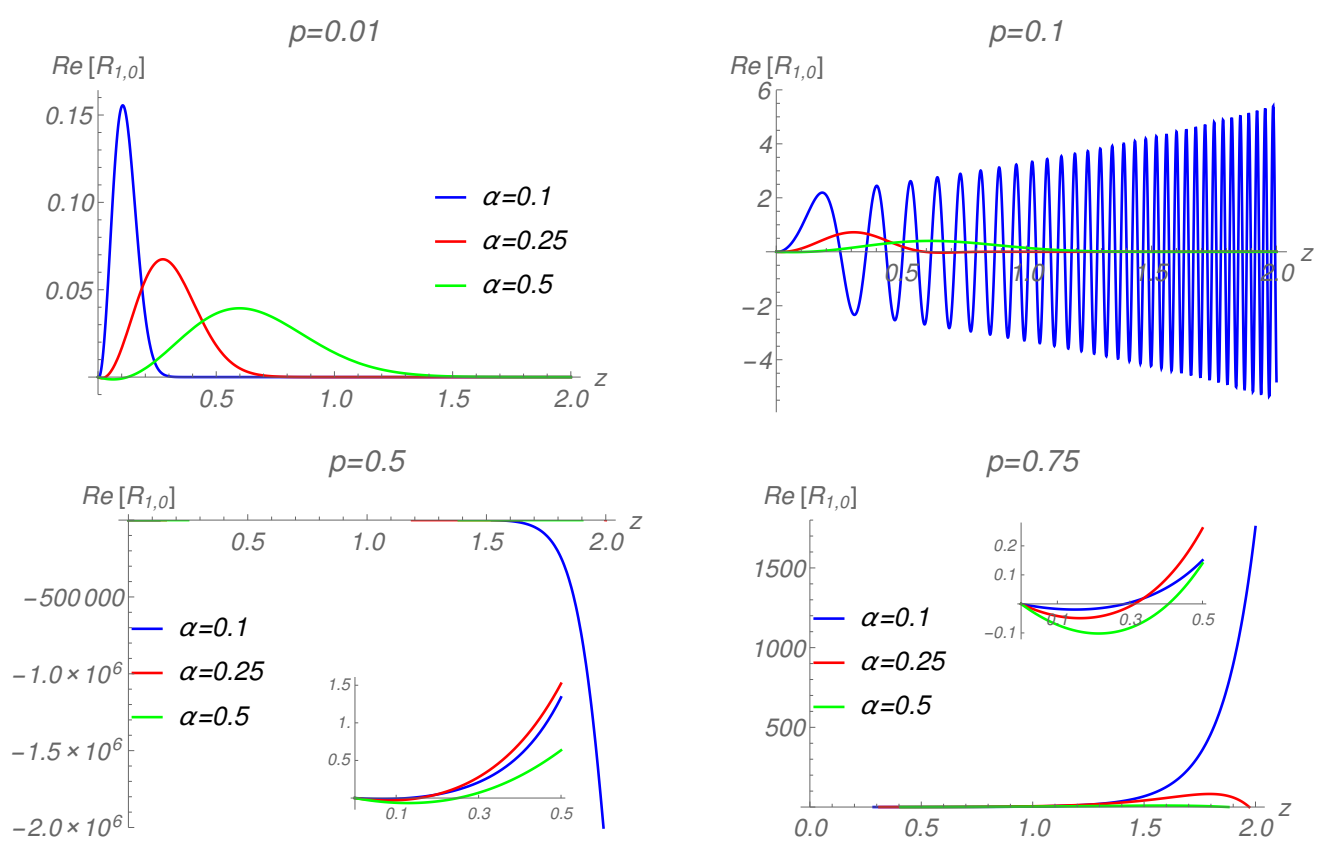

Figura 7.1: Caso I. Gráfica de Re $\left[R_{1,0}\right]$ contra $z$ para diferentes valores de $\alpha<1$ y $p<1$ en la región $0 \leq z \leq 2$.

- Caso II. Fig. (7.2). Para $0<\alpha<1$ y $1 \leq p \leq 50$. Tenemos que con $\alpha=0.1$ y $p=1$ el valor de Re $\left[R_{1,0}\right]$ explota. Por otro lado en valores de $p$ mayores a cinco se invierte el comportamiento observado anteriormente ya que el valor de $\operatorname{Re}\left[R_{1,0}\right]$ para $\alpha=0.5$ crece más que en $\alpha=0.25$ y $\alpha=0.1$. Asimismo vemos que los valores de $\operatorname{Re}\left[R_{1,0}\right]$ disminuyen al incrementar $p$ por lo que esta variable también amortigua el comportamiento oscilatorio. 

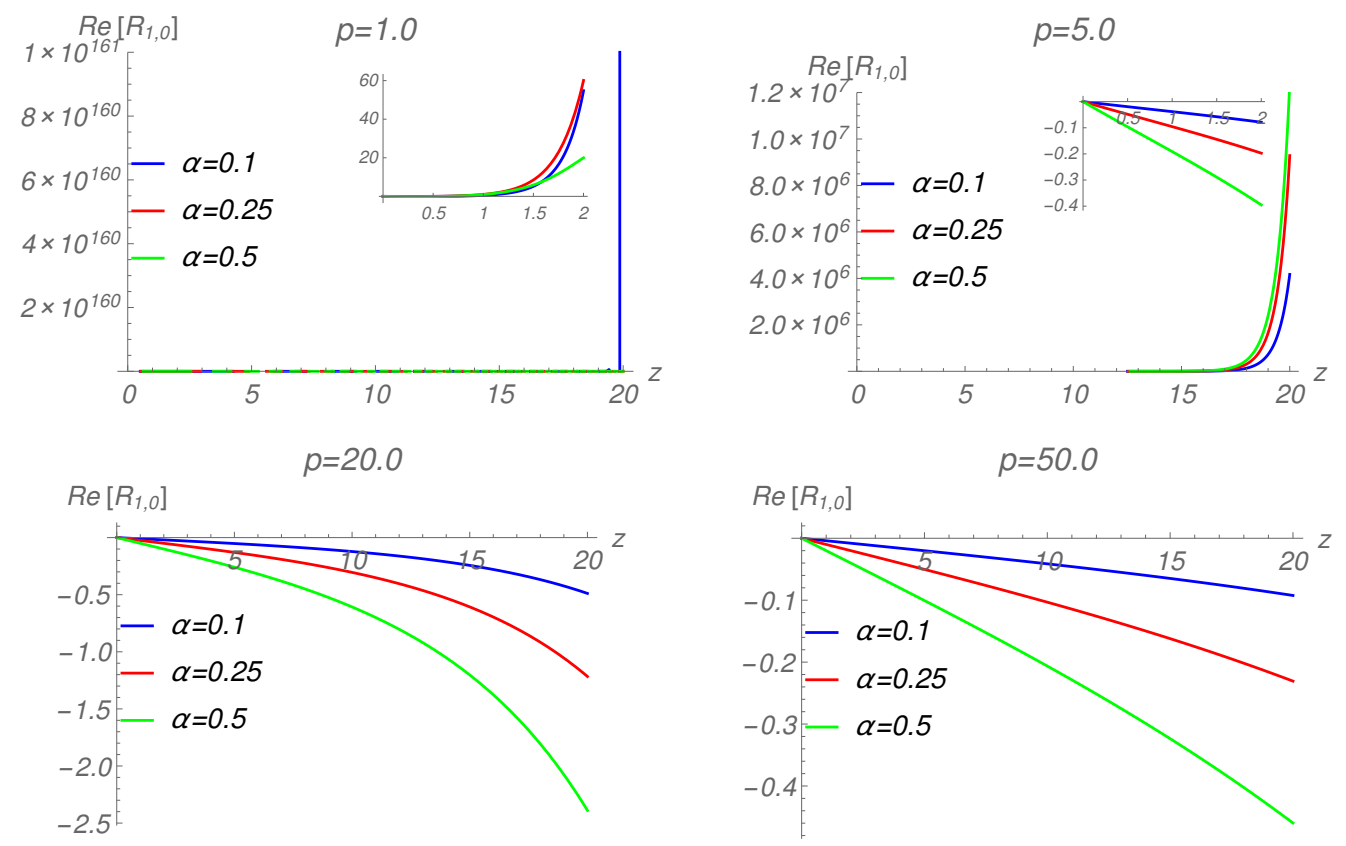

Figura 7.2: Caso II. Gráfica de Re $\left[R_{1,0}\right]$ contra $z$ para diferentes valores de $\alpha<1$ y $p \geq 1$ en la región $1 \leq z \leq 20$.

\subsubsection{2 $\alpha>1$}

Cuando $\alpha$ tiende a valores grandes $(\alpha \rightarrow \infty)$ entonces el argumento $\frac{ \pm \imath z}{p \pm \imath \alpha}$ se aproxima a valores cercanos a cero. En esta región de $\alpha$ es más factible encontrar los valores óptimos del parámetro ya que en principio se espera tener un comportamiento semejante al método asintótico.

- Caso I. Fig. (7.3). Para $5 \leq \alpha \leq 30$ y $0<p<1$. Tenemos que el valor de $\operatorname{Re}\left[R_{1,0}\right]$ para $\alpha=5$ crece más que en $\alpha=20$ y $\alpha=30$. Además los valores de Re $\left[R_{1,0}\right]$ no aumentan tanto como en la figura (7.1), también dichos valores se incrementan al hacer $p$ grande. Las curvas de esta gráfica presentan una tendencia más uniforme respecto al mismo caso en $\alpha<1$. 



Figura 7.3: Caso I. Gráfica de Re $\left[R_{1,0}\right]$ contra $z$ para diferentes valores de $\alpha>1$ y $p<1$ en la región $0 \leq z \leq 2$.

- Caso II. Fig. (7.4). Para $5 \leq \alpha \leq 30$ y $1 \leq p \leq 50$. Observamos que en $p=1$ las curvas para $\alpha=20$ y $\alpha=30$ tienen el mismo comportamiento. Conforme $p$ se incrementa los valores de Re $\left[R_{1,0}\right]$ disminuyen lo cual demuestra que $p$ contribuye con el amortiguamiento de las funciones. En general $\alpha=5$ no crece al compararse con los otros dos valores de $\alpha$.

Comparando las curvas en la Fig. (7.1) y la Fig. (7.3) para $p=0.1$ distinguimos claramente la diferencia entre $\alpha=0.1$ y $\alpha=20$ donde se confirma que la selección de valores grandes del parámetro $\alpha$ sirve para amortiguar el comportamiento de la función. 



Figura 7.4: Caso II. Gráfica de Re $\left[R_{1,0}\right]$ contra $z$ para diferentes valores de $\alpha>1$ y $p \geq 1$ en la región $1 \leq z \leq 20$.

Por lo anterior se tiene que con $\alpha>1$ los valores de Re $\left[R_{1,0}\right]$ se mantienen más acotados y uniformes para $p<1$ y $p \geq 1$. Además de que dichos valores de $\operatorname{Re}\left[R_{1,0}\right]$ se aproximan mejor a los resultados analíticos dados para cada región de $p$ estudiada. A continuación presentamos un estudio de convergencia para diferentes valores de $\alpha>1$.

En la Fig. (7.5) se presenta el comportamiento para la función $\widetilde{f}_{2,1}^{G T O}(p)$ con $\zeta=5.0$, una cuadratura de orden $N=60$ y usando tres valores diferentes del parámetro $\alpha=20, \alpha=25$ y $\alpha=30$. Tenemos que para estos valores de $\alpha$ graficados las tres curvas mantienen la misma tendencia respecto al logaritmo del error relativo en la región de $1 \leq p \leq 30$, lo cual indica que se encuentra acotado en este intervalo el valor óptimo para el parámetro $\alpha$.

La Fig. (7.6) confirma lo anterior ya que para la misma función $\widetilde{f}_{2,1}^{G T O}(p)$ con $\zeta=5.0$, orden de cuadratura $N=60$ y usando valores del parámetro $\alpha=10$ y $\alpha=40$ ambas curvas están por encima de las curvas mostradas en la Fig. (7.5). También se hicieron las mismas pruebas para las funciones $\widetilde{f}_{1,0}^{G T O}(p), \widetilde{f}_{3,2}^{G T O}(p)$ y $\widetilde{f}_{4,3}^{G T O}(p)$ encontrándose el mismo comportamiento.

Las pruebas realizadas para la transformación de las funciones de base tipo Gaussianas se hicieron considerando un valor del parámetro $\alpha=20$ esto por ser el valor de $\alpha$ más pequeño que se mantiene en el intervalo $20 \leq \alpha \leq 30$. 


$$
\tilde{f}_{2,1}^{G T O}(p), N=60
$$

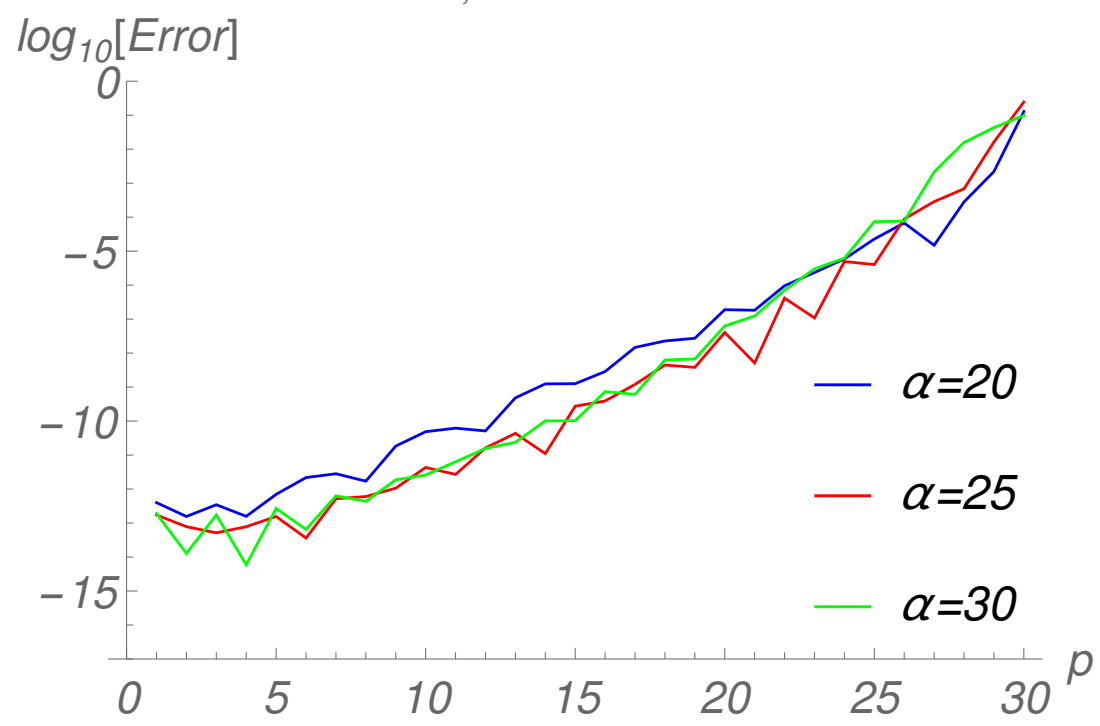

Figura 7.5: Gráfica del logaritmo del error relativo contra el valor de $p$ para la función de tipo Gaussiana con $l=1$ y $\alpha=20,25,30$ en la región de $1.0-30.0$ en $p$. 


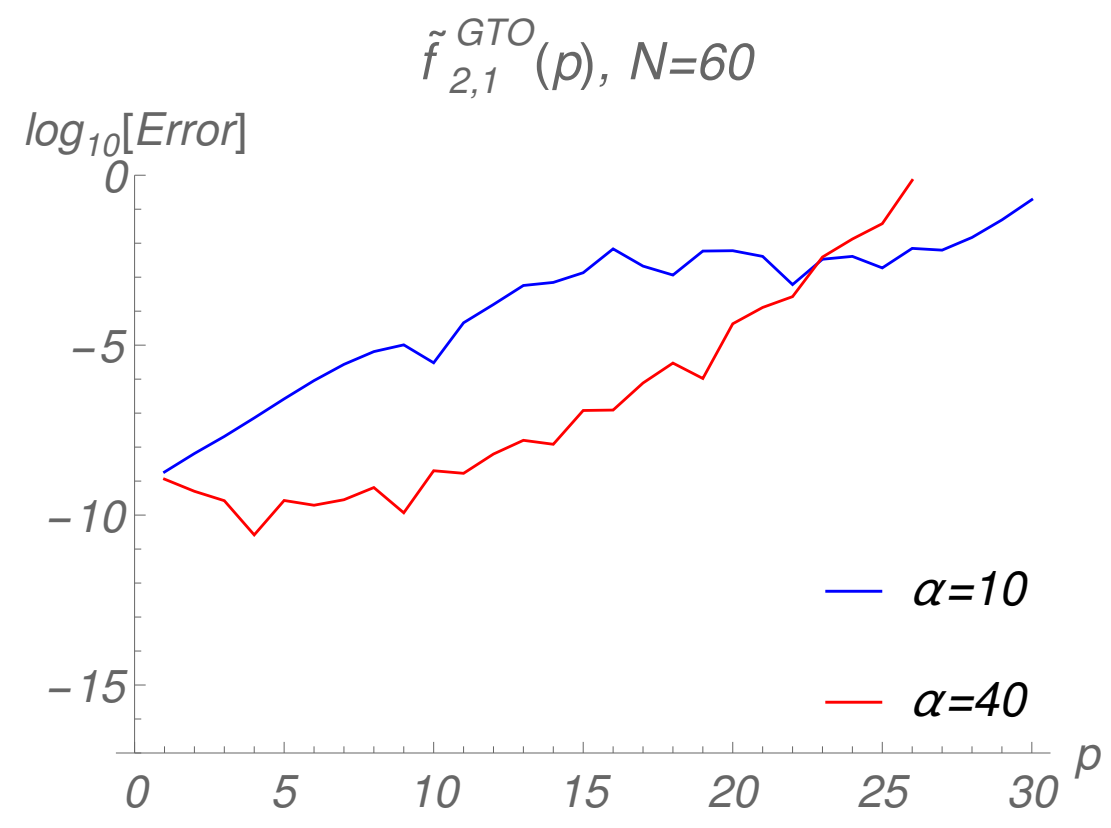

Figura 7.6: Gráfica del logaritmo del error relativo contra el valor de $p$ para la función de tipo Gaussiana con $l=1$ y $\alpha=10,40$ en la región de $1.0-30.0$ en $p$.

\subsubsection{Análisis de las sucesiones generadas en el plano complejo al evaluar el método}

En esta sección mostramos un análisis de tipo cualitativo de las sucesiones generadas en el plano complejo por la evaluación de las respectivas funciones de acuerdo al método (ver ecuaciones 7.1 .22 y 7.2 .24 ).

En la Fig. (7.7) se presentan las sucesiones generadas para funciones de base tipo Slater y Gaussianas donde usando un valor de $p=1$ en ambos casos y un parámetro $\alpha=20$ para la Gaussiana se muestra un comportamiento bastante diferenciado. En ambos casos se aprecia que existe simetría y cancelación de la parte imaginaria (eje vertical $\mathrm{Im}$ ) al sumarse los términos positivos (puntos rojos) y negativos (puntos azules). Además se observa que la gráfica del lado izquierdo correspondiente a la función de base tipo Slater $(l=0)$ barre en todos los cuadrantes y converge a cero muy rápido. Mientras que la gráfica del lado derecho correspondiente a la función de base tipo Gaussiana $(l=0)$ se mantiene acotada en el primero y cuarto cuadrante formando dos bucles que pasan por cero. 

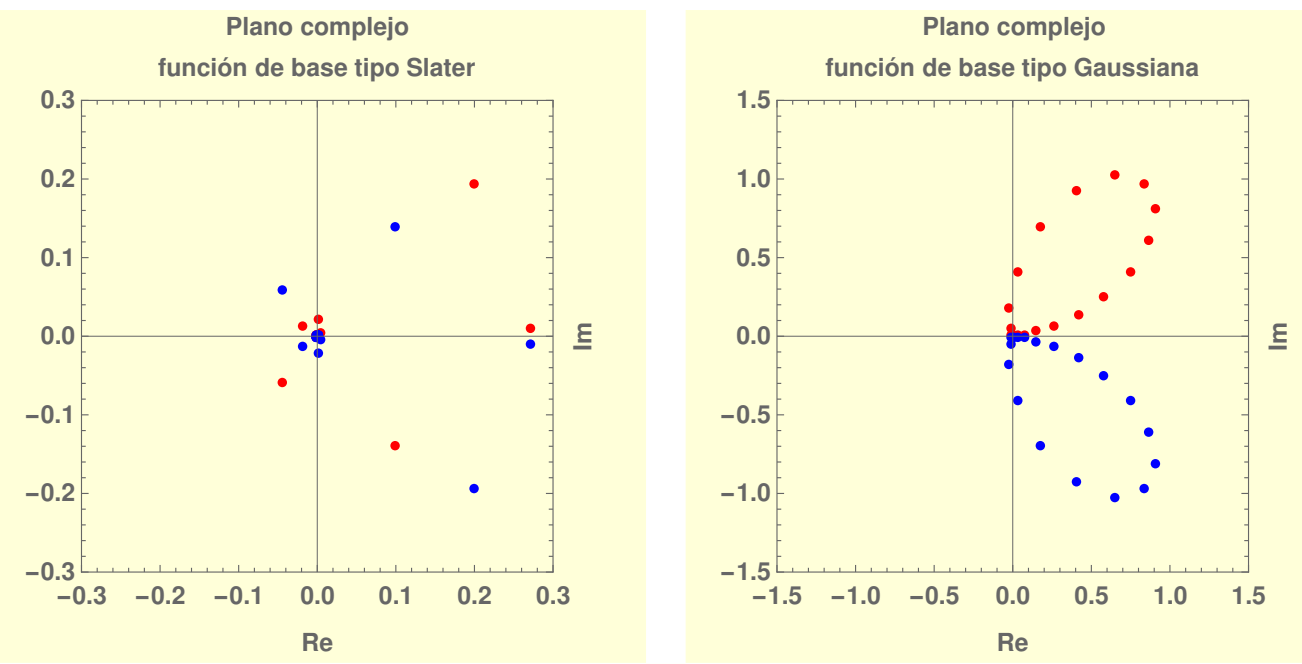

Figura 7.7: Gráfica en el plano complejo de las funciones de base tipo Slater y Gaussianas evaluadas de acuerdo al método. Tenemos en color rojo la evaluación con signo positivo y en color azul la evaluación con signo negativo para una cuadratura de orden $N=20$ y $p=1$ (Gaussiana con $\alpha=20$ ).

En la Fig (7.8) se presenta el comparativo para una función de base tipo Gaussiana usando diferentes valores del parámetro $\alpha$ para $p=1$ con una cuadratura de orden $N=20$. Para $\alpha=0$ se tiene que los valores de la sucesión son números con parte imaginaria únicamente los cuales al sumarse se cancelan y dan cero esto demuestra que el método falla sino se introduce el parámetro $\alpha$. Además observamos que conforme se incrementa el valor del parámetro se presenta una estructura más definida en la sucesión de puntos (no es dispersa como en $\alpha=1$ donde el método falla). Para los casos de $\alpha=20,30$ obtenemos la misma estructura de bucle lo cual se relaciona con las pruebas mostradas en el apartado anterior ya que al usar estos valores del parámetro se consiguen los mejores resultados. Asimismo al incrementar el valor del parámetro se pierde la estructura de bucle y el método falla. De acuerdo a esto la estructura de bucle en la sucesión de puntos está relacionada con los valores del parámetro $\alpha$ que funcionan en el método. 



Figura 7.8: Gráfica en el plano complejo de la función de base tipo Gaussiana evaluada de acuerdo al método para una cuadratura de orden $N=20$ con diferentes valores de $\alpha$ y $p=1$.

En la Fig. (7.9) se presenta el comportamiento de la sucesión al variar el valor de $p$ en la región no asintótica para $\alpha=20$. En este caso y de acuerdo a las pruebas previas (ver Fig. 7.5) al usar este valor del parámetro $\alpha$ se consiguen buenos resultados en esta región de $p$. Mientras se obtengan formas de la sucesión semejantes a una estructura de bucle (cerrado en $p=0.1,0.25$ o abierto en $p=0.5,0.75$ ) esto nos indica que el método no falla. 

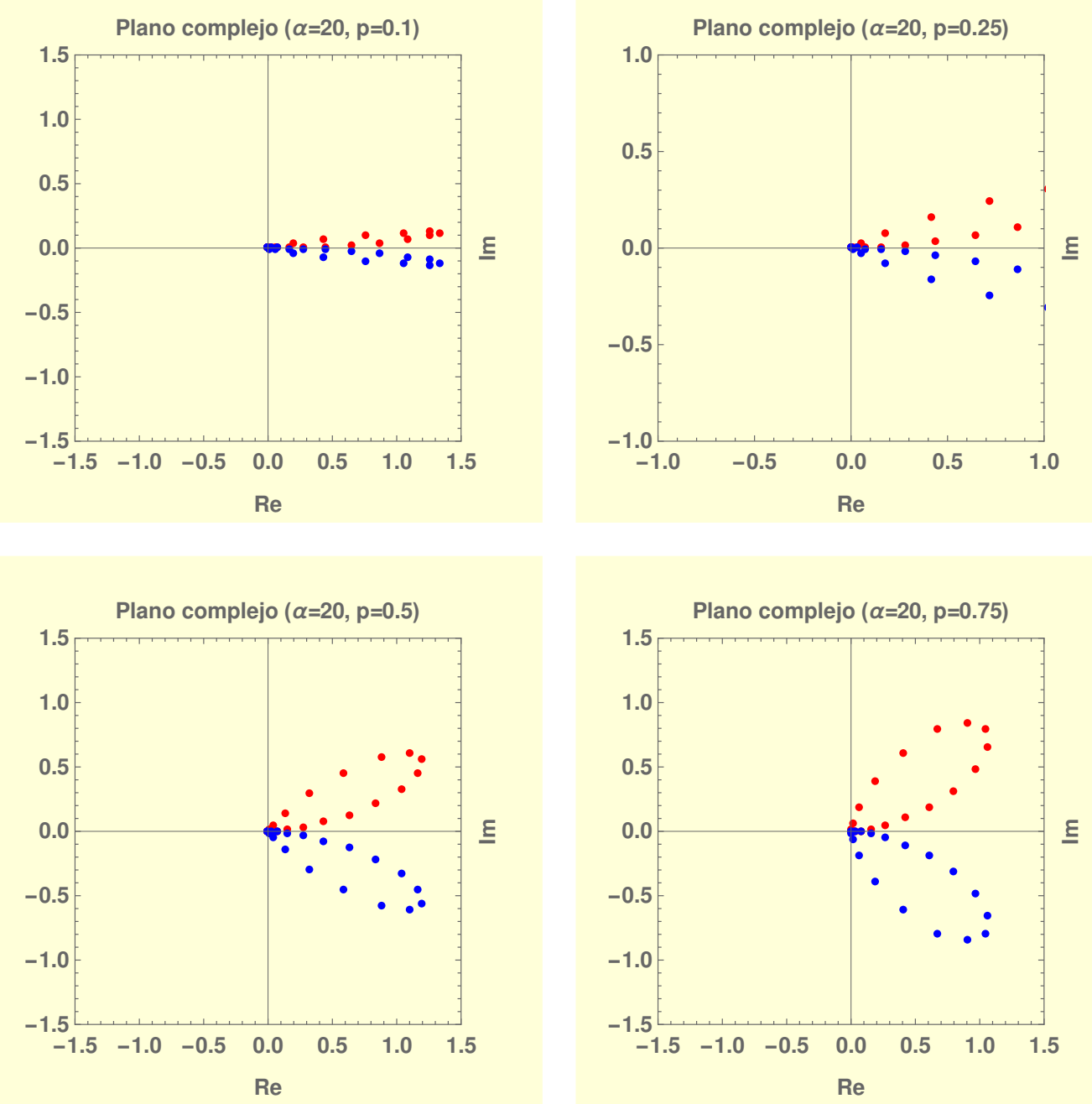

Figura 7.9: Gráfica en el plano complejo de la función de base tipo Gaussiana evaluada de acuerdo al método para una cuadratura de orden $N=20$ con valores de $\alpha=20$ y $0<p<1$.

En la Fig. (7.10) se muestra el comportamiento de la sucesión para una función de base tipo Gaussiana en la región asintótica de $p$ con $\alpha=20$. Observamos que para el valor de $p=1$ se tiene la estructura de bucle bien definida. Para $p=5,10$ se va perdiendo esta estructura y la mayor parte de los puntos pasan al segundo y tercer cuadrante, todavía en esta región el método funciona. Sin embargo para los valores de $p \geq 20$ la sucesión de puntos se va colapsando a cero y se pierde la estructura de bucle ya que el método falla completamente en esta región. 


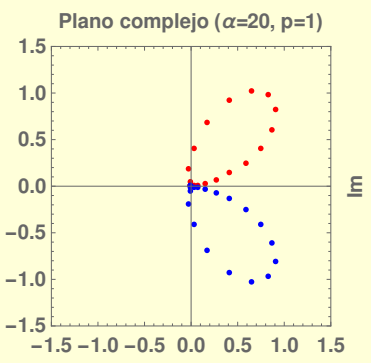

$\mathrm{Re}$

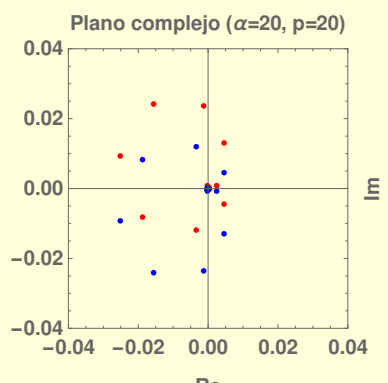

Re



$\operatorname{Re}$



$\mathrm{Re}$



Re

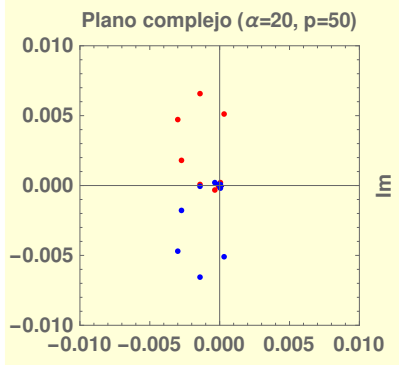

Re

Figura 7.10: Gráfica en el plano complejo de la función de base tipo Gaussiana evaluada de acuerdo al método para una cuadratura de orden $N=20$ con valores de $\alpha=20$ y $1 \leq p \leq 50$.

\subsection{Aplicación a sistemas atómicos: extensión a orbitales atómicos}

Se probó la metodología para transformar al espacio de momentos funciones de onda atómicas provenientes del método de Hartree-Fock-Roothaan [69].

Los átomos de $\mathrm{He}, \mathrm{Ne}$ y $\mathrm{Fe}$ fueron probados por lo que se usaron los valores para exponentes orbitales $\left(\zeta_{i, l}\right)$ y coeficientes de expansión lineales $\left(C_{i, l}\right)$ de las tablas que están en la referencia [70], además usamos la notación de Clementi [71]. Sabemos que el orbital $\phi_{n, l, m}$ se expande en términos de funciones de base como

$$
\phi_{n, l, m}=\sum_{i=1}^{k} C_{i, l} \chi_{i, l}
$$

donde las funciones de base tipo Slater $\chi_{i, l}$ son

$$
\chi_{i, n, l}=N_{i, l} r^{n-1} e^{-\zeta_{i, l} r}
$$


y la constante de normalización es

$$
N_{i, n, l}=\frac{\left(2 \zeta_{i, l}\right)^{n+\frac{1}{2}}}{[(2 n) !]^{\frac{1}{2}}} .
$$

\subsection{1 Átomo de Helio $(H e)$}

Para el orbital atómico $\phi(1 s)$ del Helio se usan los datos de la tabla que está en la referencia [70] donde tenemos

$$
\phi(1 s)=0.0272015 \chi_{1}+0.7407925 \chi_{2}+0.1801610 \chi_{3}+0.0798826 \chi_{4}+
$$

con funciones de base tipo Slater

$$
\begin{aligned}
& \chi_{1}=N_{1} r^{1} \exp (-1.354958 r) \\
& \chi_{2}=N_{2} r^{0} \exp (-1.455077 r) \\
& \chi_{3}=N_{3} r^{0} \exp (-2.177906 r) \\
& \chi_{4}=N_{4} r^{0} \exp (-3.384356 r) \\
& \chi_{5}=N_{5} r^{1} \exp (-6.437494 r)
\end{aligned}
$$

Para probar la metodología se utilizó la expresión completa para el orbital atómico (7.3.4) lo cual evitó calcular de manera individual cada función de base tipo Slater $\chi_{i, n, l}$ (7.3.5). Aprovechando la simetría de este orbital atómico $\phi(1 s)$ sólo necesitamos de las cuadraturas relacionadas con $l=0$ que corresponden a la función de peso $w(r)=e^{-r} \Theta_{0}(r)$.

\subsection{2 Átomo de Neón $(N e)$}

Para los orbitales atómicos $\phi(1 s)$ y $\phi(2 s)$ del Neón usamos los datos de la tabla en la referencia [70] por lo que

$$
\begin{aligned}
\phi(1 s)= & -0.0001014 \chi_{1}-0.0002064 \chi_{2}-0.0017244 \chi_{3}-0.0417988 \chi_{4}- \\
& 0.7527202 \chi_{5}-0.0891954 \chi_{6}-0.1341233 \chi_{7}-0.0005654 \chi_{8} \\
\phi(2 s)= & 0.0127644 \chi_{1}+1.0809135 \chi_{2}+0.1258193 \chi_{3}-0.4632619 \chi_{4}- \\
& 0.1044881 \chi_{5}+0.0131200 \chi_{6}+0.0046073 \chi_{7}-0.0001682 \chi_{8}
\end{aligned}
$$

que tienen funciones de base tipo Slater 


$$
\begin{aligned}
& \chi_{1}=N_{1} r^{0} \exp (-1.304155 r) \\
& \chi_{2}=N_{2} r^{0} \exp (-2.016153 r) \\
& \chi_{3}=N_{3} r^{1} \exp (-3.574219 r) \\
& \chi_{4}=N_{4} r^{0} \exp (-6.491668 r) \\
& \chi_{5}=N_{5} r^{0} \exp (-9.144899 r) \\
& \chi_{6}=N_{6} r^{1} \exp (-13.516489 r) \\
& \chi_{7}=N_{7} r^{0} \exp (-16.354484 r) \\
& \chi_{8}=N_{8} r^{1} \exp (-29.214419 r)
\end{aligned}
$$

En este caso también se usaron las expresiones de los orbitales atómicos completos lo cual evitó calcular de manera individual cada función de base tipo Slater. Aprovechando la simetría de estos orbitales atómicos $\phi(1 s)$ y $\phi(2 s)$ requerimos de las cuadraturas relacionadas con $l=0$ correspondientes a la función de peso $w(r)=e^{-r} \Theta_{0}(r)$

Para el orbital atómico $\phi(2 p)$ del Neón usamos los datos de la tabla en la referencia [70] donde tenemos que

$$
\begin{array}{r}
\phi(2 p)=0.0510413 \chi_{1}+0.3203928 \chi_{2}+0.3958489 \chi_{3}+0.2801866 \chi_{4}+ \\
0.0340866 \chi_{5}+0.0203038 \chi_{6}+0.0000409 \chi_{7}
\end{array}
$$

con funciones de base tipo Slater

$$
\begin{aligned}
& \chi_{1}=N_{1} r^{1} \exp (-1.304155 r) \\
& \chi_{2}=N_{2} r^{1} \exp (-1.710436 r) \\
& \chi_{3}=N_{3} r^{1} \exp (-2.648660 r) \\
& \chi_{4}=N_{4} r^{1} \exp (-4.295590 r) \\
& \chi_{5}=N_{5} r^{2} \exp (-8.124569 r) \\
& \chi_{6}=N_{6} r^{1} \exp (-10.674843 r) \\
& \chi_{7}=N_{7} r^{2} \exp (-25.731219 r)
\end{aligned}
$$

Utilizamos la expresión para el orbital atómico completo lo cual evita calcular de manera individual cada función de base tipo Slater. Aprovechamos la simetría de este orbital atómico $\phi(2 p)$ ya que sólo necesitamos de las cuadraturas relacionadas con $l=1$ siendo la función de peso $w(r)=$ $e^{-r} \Theta_{1}(r)$. 


\subsection{3 Átomo de Hierro $(\mathrm{Fe})$}

Para el orbital atómico $\phi(3 d)$ del Hierro usamos los datos de la tabla que aparece en la referencia [70]

$$
\begin{array}{r}
\phi(3 d)=0.0003175 \chi_{1}+0.0891893 \chi_{2}+0.2865224 \chi_{3}+0.3855914 \chi_{4}+ \\
0.2946262 \chi_{5}+0.0981612 \chi_{6}+0.0069661 \chi_{7}+0.0016054 \chi_{8}
\end{array}
$$

con funciones de base tipo Slater

$$
\begin{aligned}
& \chi_{1}=N_{1} r^{2} \exp (-0.718583 r) \\
& \chi_{2}=N_{2} r^{2} \exp (-1.404987 r) \\
& \chi_{3}=N_{3} r^{2} \exp (-2.122232 r) \\
& \chi_{4}=N_{4} r^{2} \exp (-3.309121 r) \\
& \chi_{5}=N_{5} r^{2} \exp (-5.208309 r) \\
& \chi_{6}=N_{6} r^{2} \exp (-7.891984 r) \\
& \chi_{7}=N_{7} r^{3} \exp (-18.020857 r) \\
& \chi_{8}=N_{8} r^{2} \exp (-23.292198 r)
\end{aligned}
$$

También se utilizó la expresión para el orbital atómico completo lo cual evita calcular de manera individual cada función de base tipo Slater. Considerando la simetría de este orbital atómico $\phi(3 d)$ sólo requerimos de las cuadraturas relacionadas con $l=2$ donde la función de peso es $w(r)=e^{-r} \Theta_{2}(r)$.

\subsection{Discusión de resultados}

\subsubsection{Funciones de base tipo Slater}

Los resultados para las funciones de base tipo Slater se agrupan y analizan de acuerdo al valor de $l$ ya que controla el orden de la transformada de Bessel esférica. Este método utiliza una cuadratura diferente para cada valor de $l$.

Las gráficas se dividen para analizar el funcionamiento del método en dos regiones. La región asintótica $1 \leq p \leq 50$ y la región no asintótica $0<p \leq 1$. Se estudia también la convergencia del método con respecto al orden de la cuadratura $N$ y los valores de $p$ en la región no asintótica. Se presentan las gráficas de una función por cada valor de $l$. Además se tiene que los resultados y el comportamiento son representativos para otras funciones con el mismo valor de $l$.

Usamos una nueva metodología para calcular numéricamente estas integrales que transforman funciones radiales al espacio de momentos empleando un código escrito en FORTRAN. Se calcularon las integrales para 
funciones radiales con $l=0, l=1, l=2, l=3$ y $l=4$. Los cálculos se hicieron utilizando aritmética en precisión doble para diferentes valores de p. Los resultados numéricos se comparan con los valores que se obtienen a partir de las expresiones analíticas, ver Tabla (7.1). Las gráficas se generaron calculando valores con pasos de una unidad en la región $1 \leq p \leq 50$ y 0.02 unidades en la región $0<p \leq 1$. Graficamos el logaritmo base 10 del valor absoluto del error relativo $\log _{10}[$ Error $]$ contra los valores de $p$ donde el error relativo se ha definido en (6.3.1).

\subsubsection{1 $l=0$}

En la Fig. (7.11) se muestra el error como una función de $p$ la cual se obtiene a partir de las cuadraturas de orden $N=5$ y $N=10$ basadas en la función de peso $e^{-r} \Theta_{0}(r)$. Las gráficas del cálculo de $\widetilde{f}_{1,0}^{S T O}(p)$ muestran que el método funciona mejor (el error disminuye) a medida que $p$ aumenta para un orden de cuadratura particular. Tomando en cuenta que se pueden obtener buenos resultados para $p$ mayor con tan sólo una cuadratura de cinco puntos. Esto muestra que se comporta como el método de Wong usado para la transformada de Hankel.

El método funciona mejor en regiones grandes del valor de $p$ donde los métodos convencionales tienen problemas debido a la naturaleza oscilatoria del integrando. La cuadratura de diez puntos produce resultados esencialmente exactos para $p>5$.

El mejor funcionamiento del método para $p$ mayor con una cuadratura de orden inferior significa que el integrando debe comportarse como un polinomio de orden inferior para $p$ mayor. Esto se puede entender a partir de (7.1.22). $\tilde{f}_{1,0}^{S T O}(p)$ es una función de valor real por lo que los componentes imaginarios de la ecuación (7.1.22) deben cancelarse. El comportamiento de la componente real del integrando en el primer término para diferentes valores de $p$ se presenta en la Fig. (7.12). Se observa que el comportamiento oscilatorio del integrando para $p$ menor se amortigua a medida que $p$ aumenta. De hecho la función se asemeja a un polinomio de orden inferior para $p$ mayor. El integrando del segundo término de la ecuación (7.1.22) muestra el mismo comportamiento. 




Figura 7.11: Gráfica del logaritmo del error relativo contra el valor de $p$ para la función tipo Slater con $l=0$ en la región de $1.0-50.0$ en $p$. 


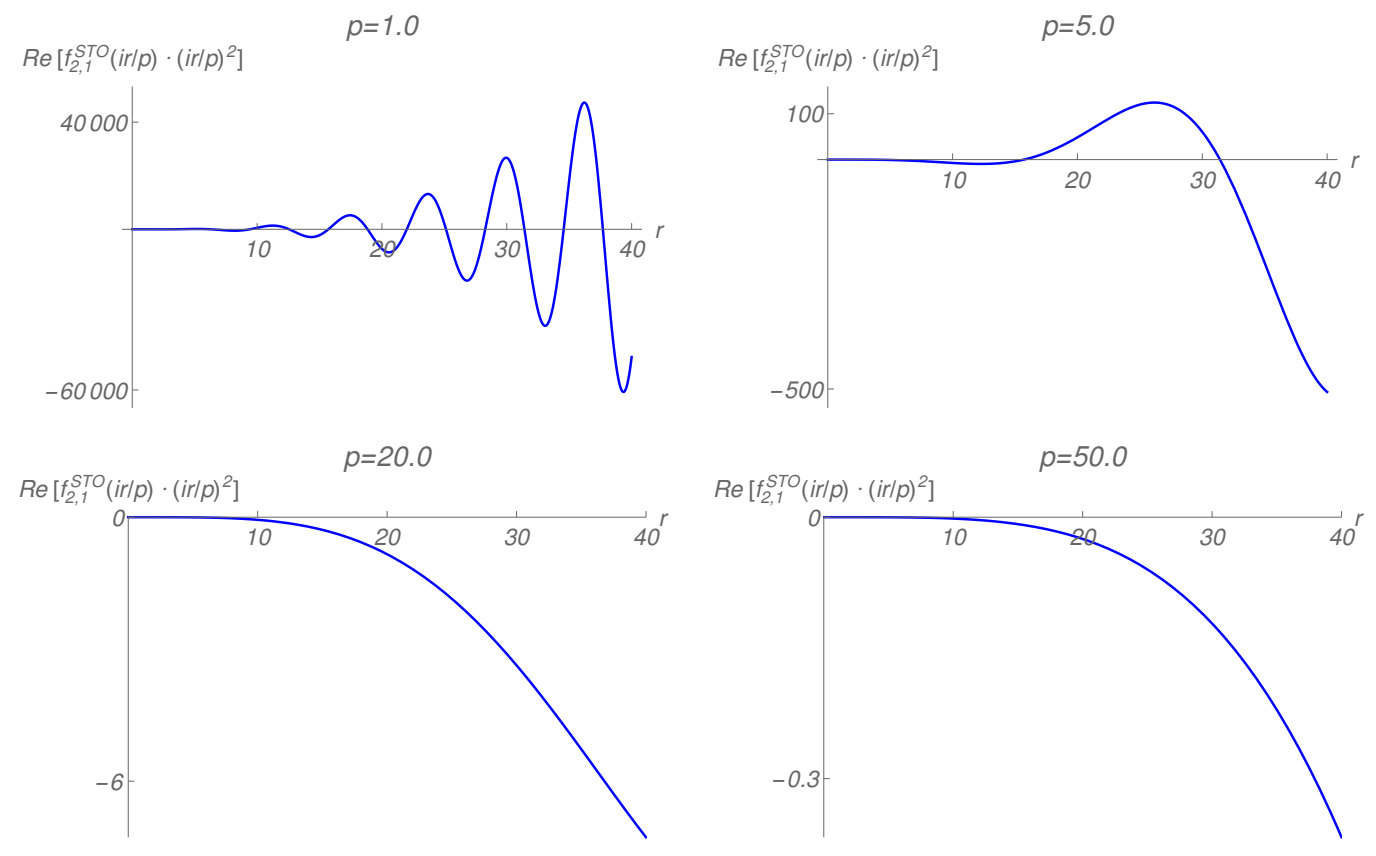

Figura 7.12: Gráfica de $\operatorname{Re}\left[f_{2,1}^{S T O}(\imath r / p) \cdot(\imath r / p)^{2}\right]$ contra $r$ para diferentes valores de $p$.

En la Fig. (7.13) se presenta el análisis del error con diferentes órdenes de cuadratura para $0<p \leq 1$. Se observa que cuadraturas de orden superior tienen que ser empleadas en esta región con el fin de obtener resultados razonables. Obtenemos mejores resultados aumentando el orden de la cuadratura. Además enfatizamos que el método falla para valores de $p<0.2$.

Un estudio del error frente al orden de la cuadratura para $p=0.5$ se muestra en la Fig. (7.14), donde se ve que pueden conseguirse resultados confiables aumentando el orden de la cuadratura. 


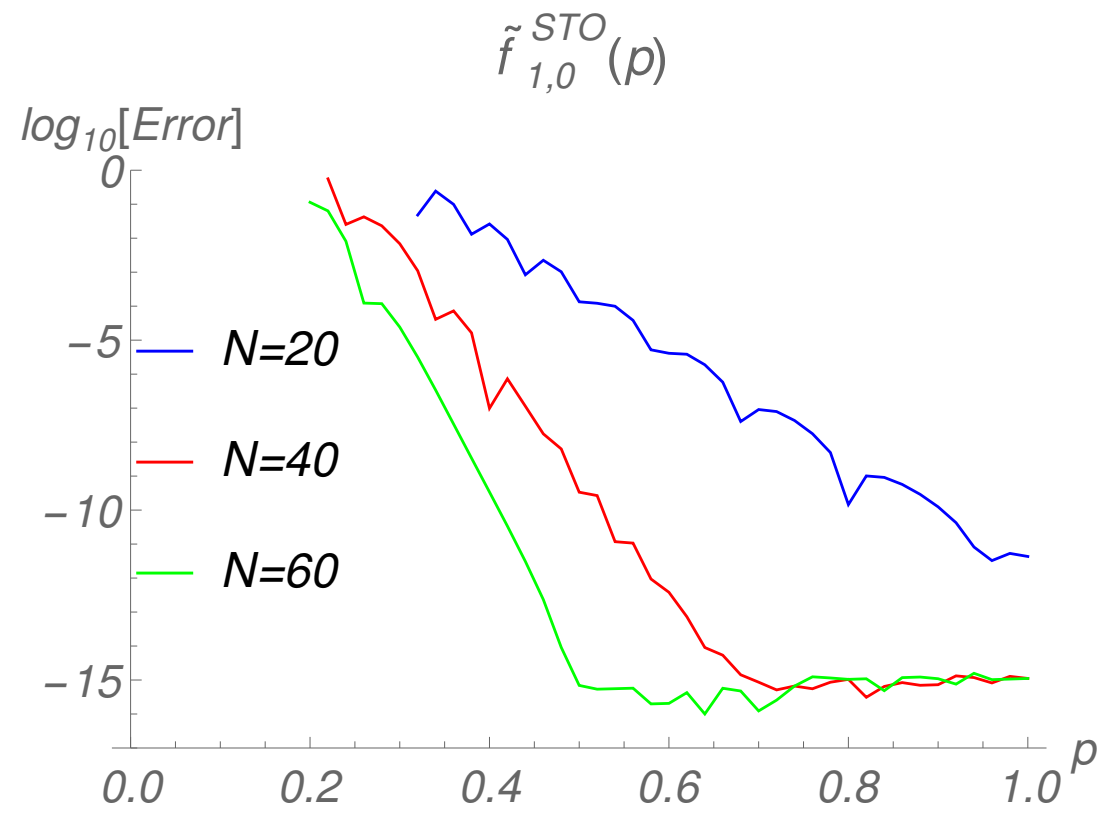

Figura 7.13: Gráfica del logaritmo del error relativo contra el valor de $p$ para la función tipo Slater con $l=0$ en la región de $0.0-1.0$ en $p$.

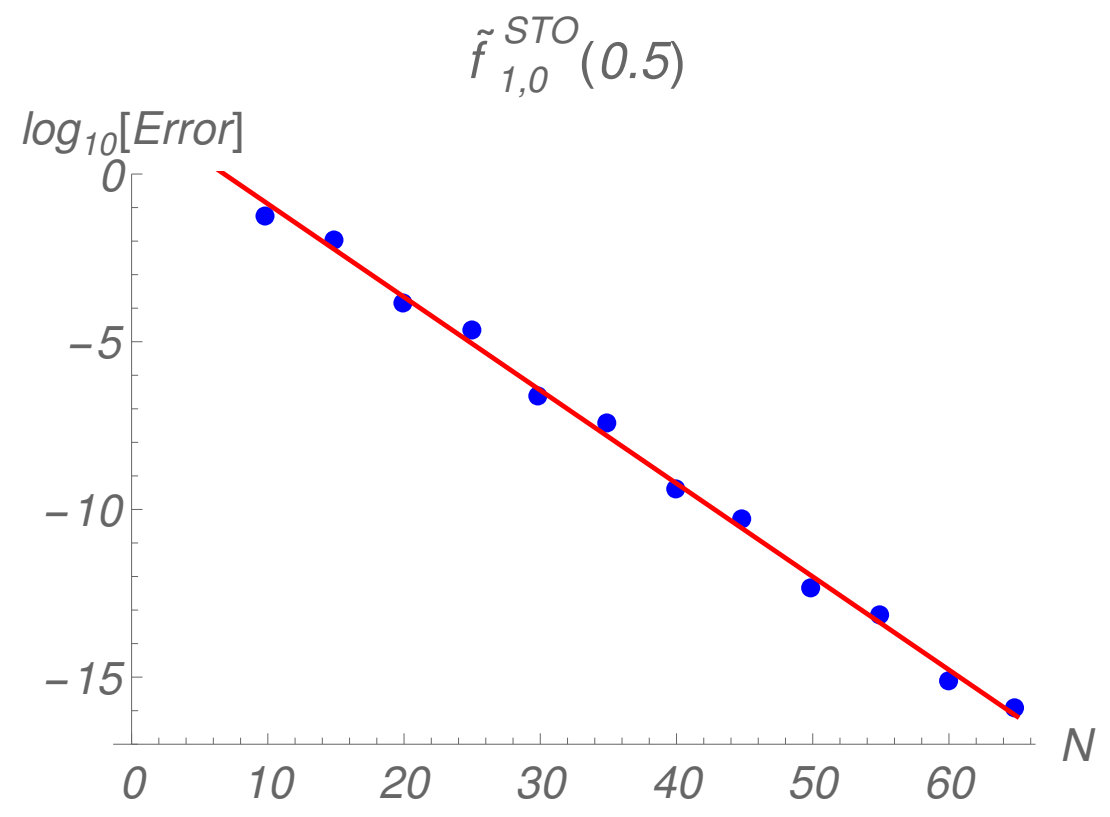

Figura 7.14: Gráfica del logaritmo del error relativo contra el orden de la cuadratura $N$ para la función con $l=0$ en $p=0.5$. 


\subsubsection{2 $l=1$}

En esta parte estudiamos el funcionamiento de las cuadraturas basadas en la función de peso $e^{-r} \Theta_{1}(r)$. Debemos señalar que la función $\widetilde{f}_{2,1}^{S T O}(p)$ de tipo $l=1$ es de valor complejo pero al realizar el cálculo del error relativo los valores de $\imath$ que aparecen se cancelan.

La Fig. (7.15) presenta las gráficas para la función $l=1$ donde se observan resultados similares a los de la función $l=0$. La cuadratura de cinco puntos produce valores exactos para $p>50$. Del mismo modo la cuadratura de diez puntos produce resultados exactos para $5<p<50$.

Una observación adicional para la gráfica con $N=5$ y $N=10$ es que con estas cuadraturas se logran resultados menos exactos para valores más pequeños de $p$ en comparación con las cuadraturas $e^{-r} \theta_{0}(r)$ de cinco y diez puntos para $l=0$ como en la Fig. (7.11).

Los resultados para valores pequeños de $p(0<p \leq 1)$ se presentan en la Fig. (7.16) donde obtenemos excelentes resultados con una cuadratura de $N=60$ para $p>0.5$. No se pueden obtener resultados significativos para $p<0.2$ con las cuadraturas estudiadas.

Por otro lado la Fig. (7.17) para $p=0.5$ ilustra que los resultados se pueden mejorar elevando el orden de la cuadratura. Sin embargo existe un problema ya que esta convergencia no es monótona por lo que presentamos la Fig. (7.18) para $p=0.7$ la cual sí presenta una convergencia monótona. Esto demuestra que la convergencia monótona es dependiente del valor de $p$ seleccionado aunque en ambos casos se observa que existe convergencia. 


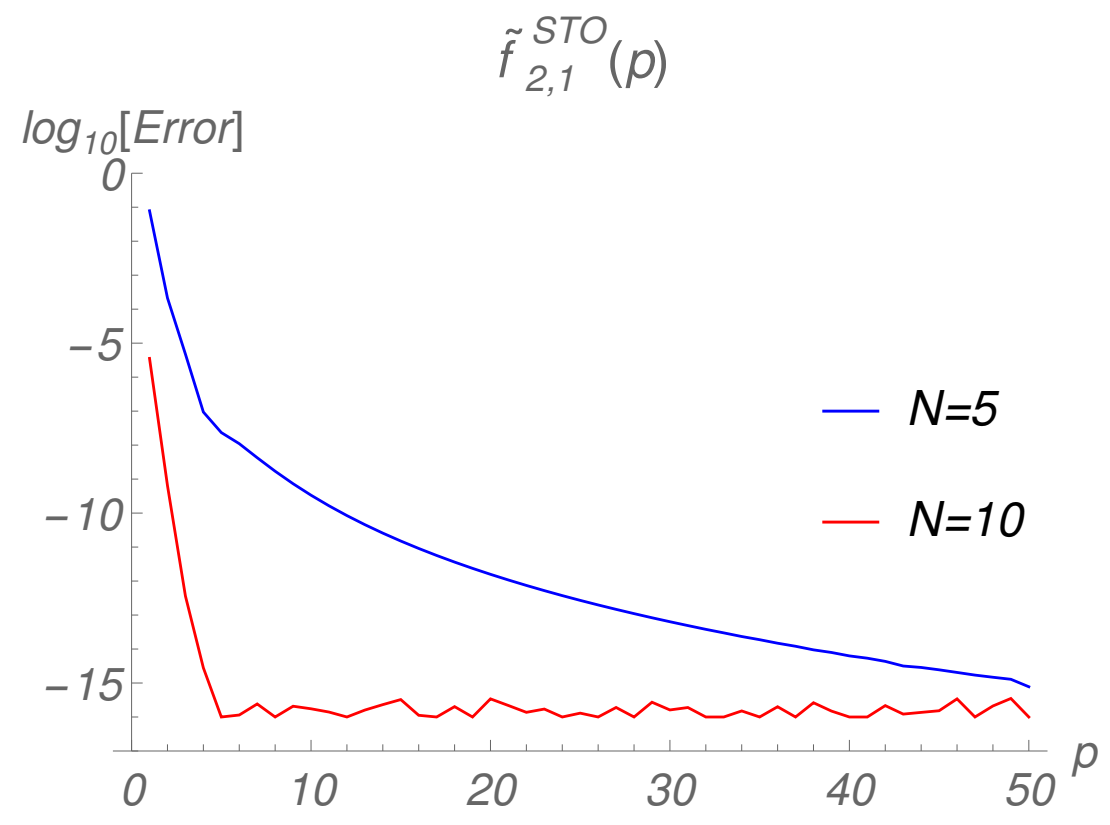

Figura 7.15: Gráfica del logaritmo del error relativo contra el valor de $p$ para la función tipo Slater con $l=1$ en la región de $1.0-50.0$ en $p$.

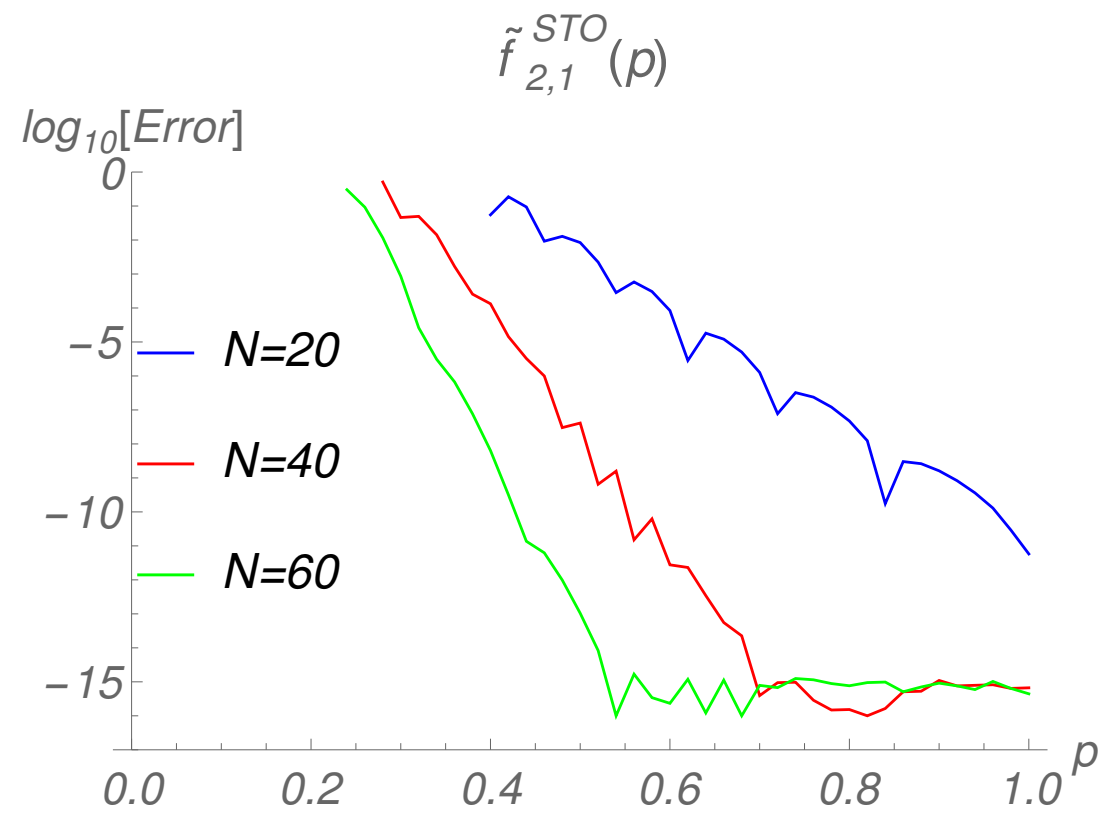

Figura 7.16: Gráfica del logaritmo del error relativo contra el valor de $p$ para la función tipo Slater con $l=1$ en la región de $0.0-1.0$ en $p$. 


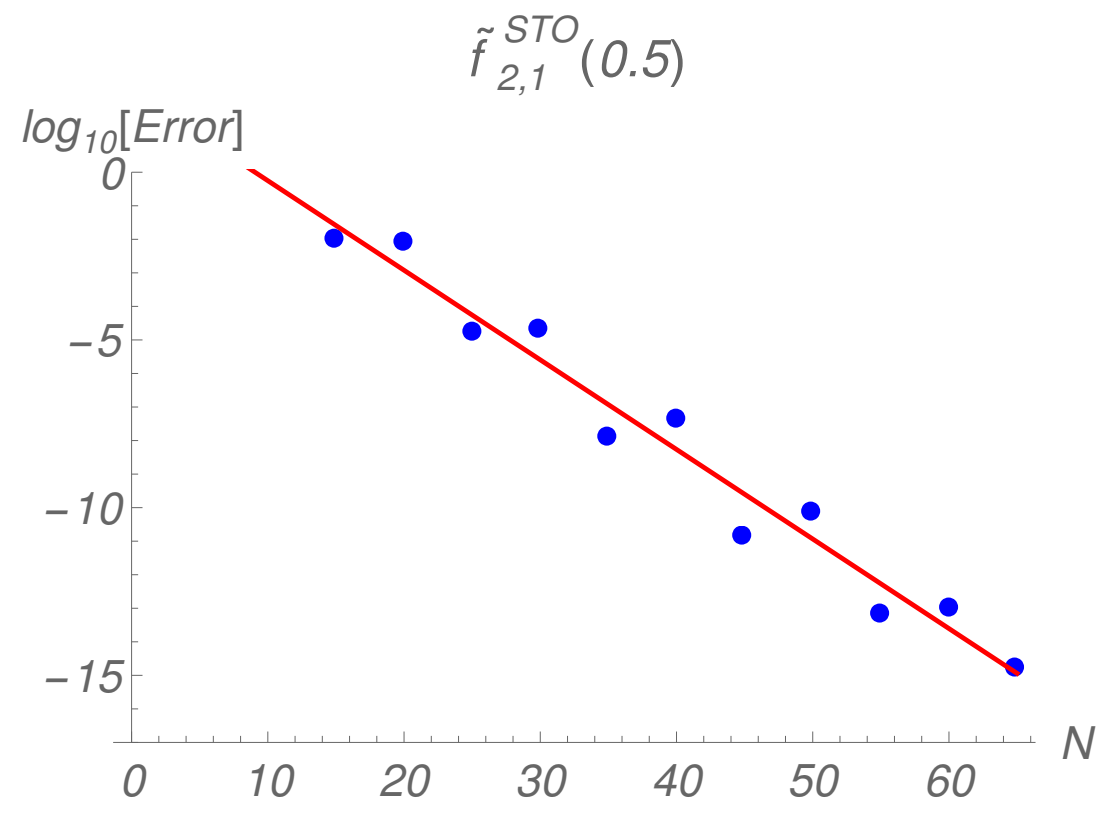

Figura 7.17: Gráfica del logaritmo del error relativo contra el orden de la cuadratura $N$ para la función con $l=1$ en $p=0.5$.

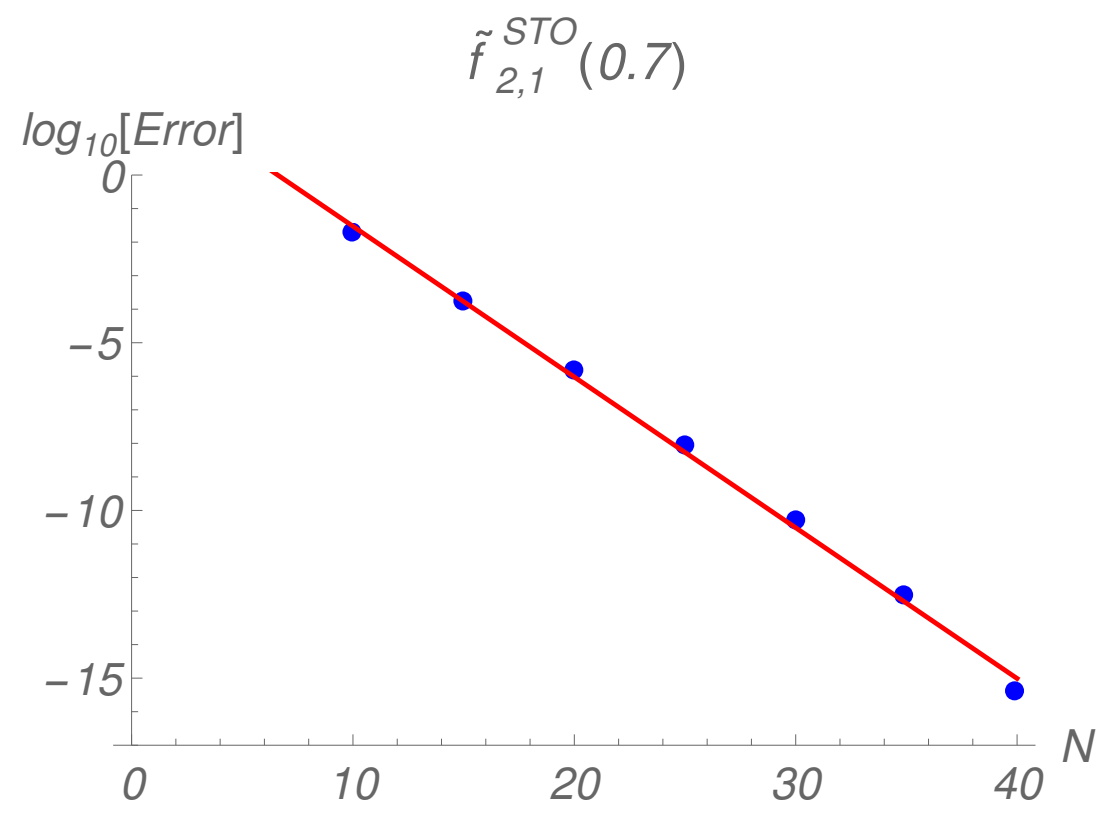

Figura 7.18: Gráfica del logaritmo del error relativo contra el orden de la cuadratura $N$ para la función con $l=1$ en $p=0.7$. 


\subsubsection{3 $l=2$}

Ahora estudiamos el funcionamiento de las cuadraturas basadas en la función de peso $e^{-r} \Theta_{2}(r)$ donde usamos la función $\widetilde{f}_{3,2}^{S T O}(p)$ de tipo $l=2$.

La Fig. (7.19) presenta las gráficas para la función $l=2$ donde se observan resultados similares a los de las funciones $l=0 \mathrm{y} l=1$. También notamos que la cuadratura de cinco puntos produce valores exactos para $p>50$. Asimismo la cuadratura de diez puntos produce resultados exactos para $5<p<50$.

Para la gráfica con cuadraturas $N=5$ y $N=10$ se logran resultados menos exactos para valores más pequeños de $p$ en comparación con las cuadraturas de cinco y diez puntos para $l=0$ y $l=1$ como vemos en la Fig. (7.11).

Los resultados para valores pequeños de $p(0<p \leq 1)$ se presentan en la Fig. (7.20) donde conseguimos excelentes resultados con una cuadratura de $N=60$ para $p>0.55$. No se pueden obtener resultados significativos para $p<0.2$ con las cuadraturas estudiadas. Por otro lado la Fig. (7.21) para $p=0.5$ ilustra que los resultados se pueden mejorar elevando el orden de la cuadratura.

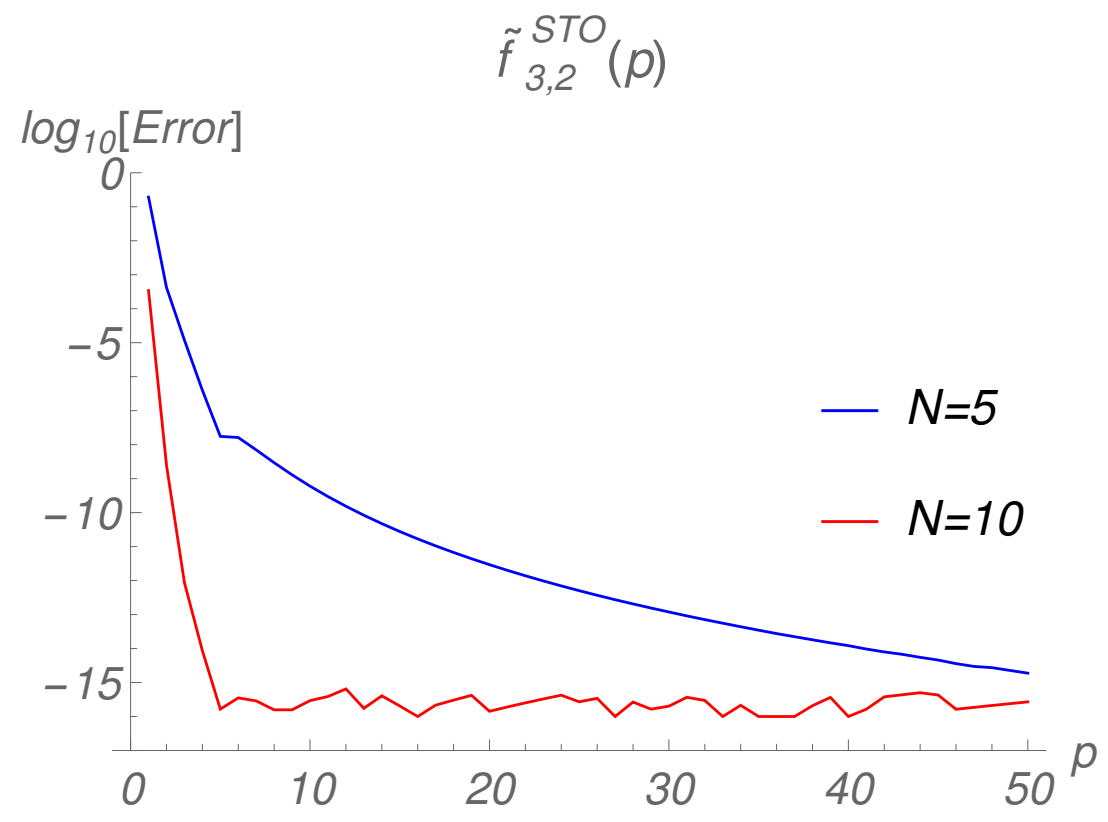

Figura 7.19: Gráfica del logaritmo del error relativo contra el valor de $p$ para la función tipo Slater con $l=2$ en la región de $1.0-50.0$ en $p$. 


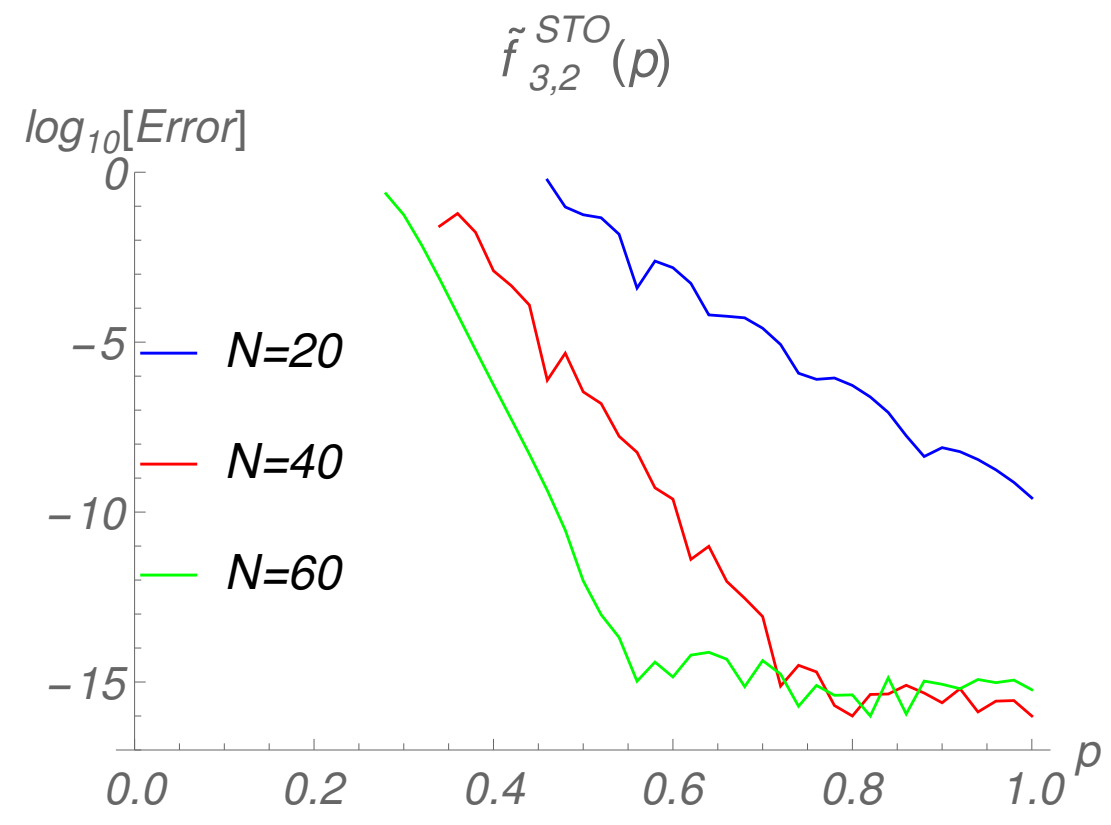

Figura 7.20: Gráfica del logaritmo del error relativo contra el valor de $p$ para la función tipo Slater con $l=2$ en la región de $0.0-1.0$ en $p$.



Figura 7.21: Gráfica del logaritmo del error relativo contra el orden de la cuadratura $N$ para la función con $l=2$ en $p=0.5$. 


\subsubsection{4 $l=3,4$}

Los resultados obtenidos de las cuadraturas basadas en la función de peso $e^{-r} \Theta_{4}(r)$ se presentan en las Figs. (7.22 - 7.23) para la función de prueba $\widetilde{f}_{5,4}^{S T O}(p)$. También se estudiaron las gráficas pertenecientes a los resultados de las cuadraturas con la función de peso $e^{-r} \Theta_{3}(r)$ pero no se presentan, las cuales muestran un comportamiento similar a las gráficas mostradas para $l=4$.

Los resultados son similares a los presentados para $l=0,1,2$. La cuadratura de cinco puntos no da buenos resultados en este intervalo ya que no mejora para $p<50$ mientras que la cuadratura de diez puntos produce resultados esencialmente exactos para $p>5$. La cuadratura en orden $N=60$ proporciona buenos resultados para $p>0.7$. El error aumenta rápidamente con $p<0.7$ por lo que el método se esfuerza por proporcionar resultados precisos en esta región.

La Fig. (7.24) muestra la convergencia del método con el orden de cuadratura en $p=0.5$. Lo cual demuestra que se pueden obtener mejores resultados aumentando el orden de la cuadratura.

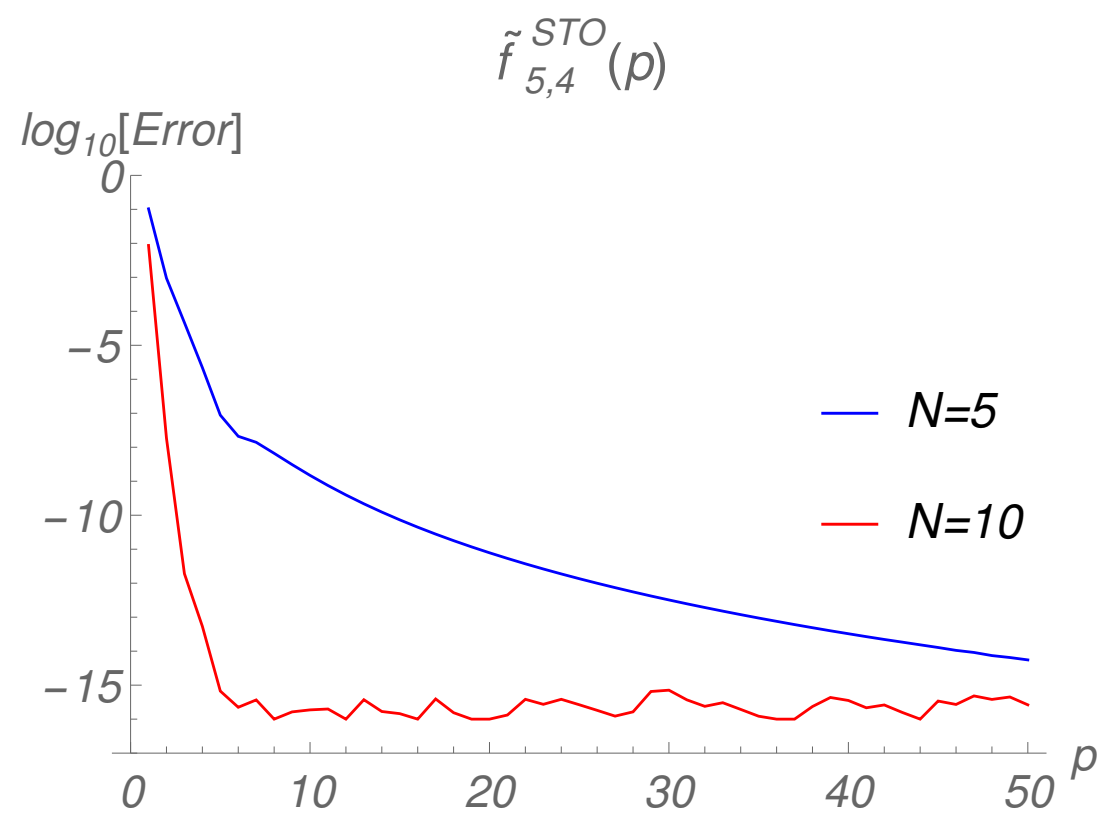

Figura 7.22: Gráfica del logaritmo del error relativo contra el valor de $p$ para la función tipo Slater con $l=4$ en la región de $1.0-50.0$ en $p$. 


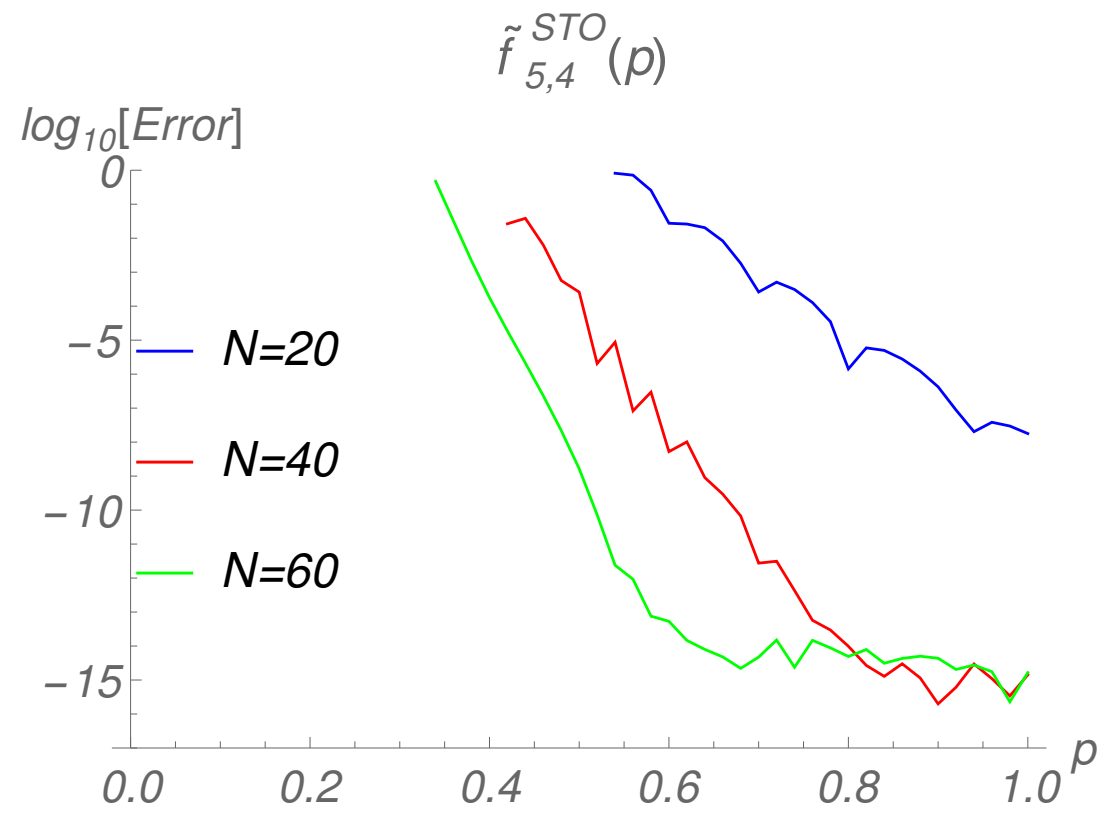

Figura 7.23: Gráfica del logaritmo del error relativo contra el valor de $p$ para la función tipo Slater con $l=4$ en la región de $0.0-1.0$ en $p$.

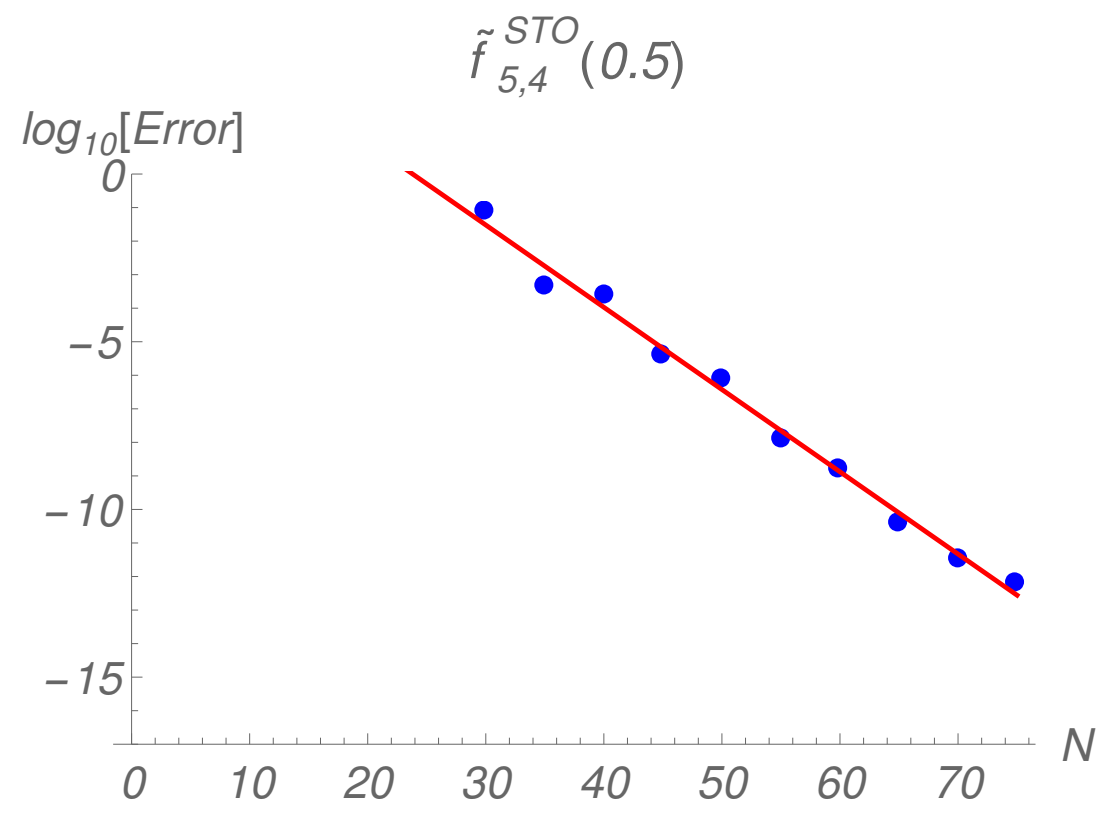

Figura 7.24: Gráfica del logaritmo del error relativo contra el orden de la cuadratura $N$ para la función con $l=4$ en $p=0.5$. 


\subsubsection{Funciones de base tipo Gaussianas}

La nueva metodología estudiada falla al calcular la transformada de Bessel esférica en funciones de base tipo Gaussianas si no se introduce el parámetro $\alpha$ que rompe la simetría. Para mostrar el funcionamiento del método estudiamos funciones con $l=0, l=1, l=2$ y $l=3$ en las cuales usamos valores de $\zeta=5$ y $\alpha=20$. Además se establecen las mismas condiciones de prueba que previamente fueron consideradas para las funciones de base tipo Slater. En este caso no analizamos la convergencia por el hecho de que el funcionamiento del método depende de un parámetro. Las funciones con $l=1$ y $l=3$ dan valores complejos pero las $\imath$ que aparecen se cancelan al calcular el error relativo. A continuación presentamos los resultados obtenidos con la introducción de $\alpha$ en esta nueva metodología (7.2.1)

\subsubsection{1 $l=0$}

En la Fig. (7.25) se muestra el error como una función de $p$ la cual obtenemos a partir de las cuadraturas de orden $N=20$ y $N=40$ basadas en la función de peso $e^{-r} \Theta_{0}(r)$. Las gráficas del cálculo de $\widetilde{f}_{1,0}^{G T O}(p)$ muestran que el método funciona mejor (el error disminuye) a medida que $p$ disminuye para un orden de cuadratura particular. En este caso no podemos conseguir buenos resultados para $p$ grandes. Esto muestra que el método no se comporta de manera asintótica al introducir el parámetro y es distinto al comportamiento del Slater.

El método se desempeña mejor en regiones pequeñas del valor de $p$ donde los métodos convencionales funcionan. La cuadratura de cuarenta puntos produce resultados esencialmente exactos para $p<15$.

En la Fig. (7.26) presentamos el análisis del error con diferentes órdenes de cuadratura para $0<p \leq 1$. Se observa que con un orden de cuadratura $N=20$ conseguimos resultados razonables obteniéndose valores exactos al incrementar el orden de la cuadratura. Además se enfatiza que el método no falla en esta región. 


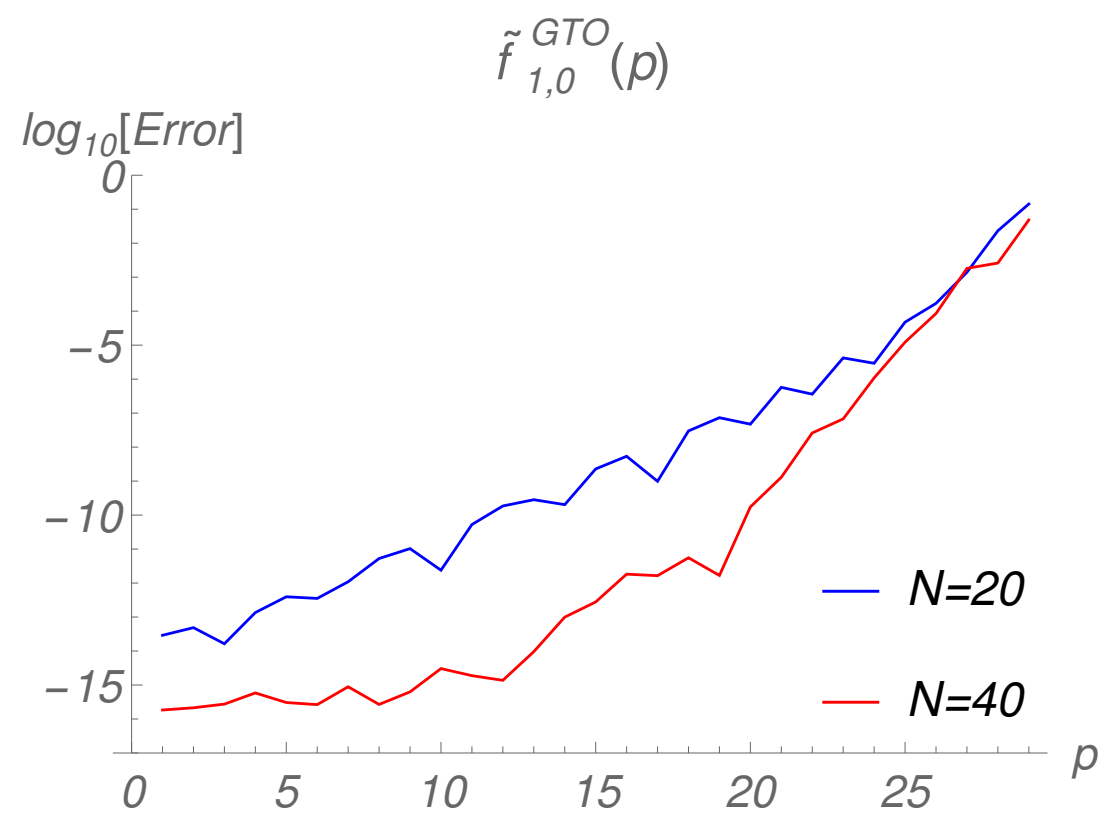

Figura 7.25: Gráfica del logaritmo del error relativo contra el valor de $p$ para la función tipo Gaussiana con $l=0$ y $\zeta=5.0$ en la región de $1.0-29.0$ en $p$.

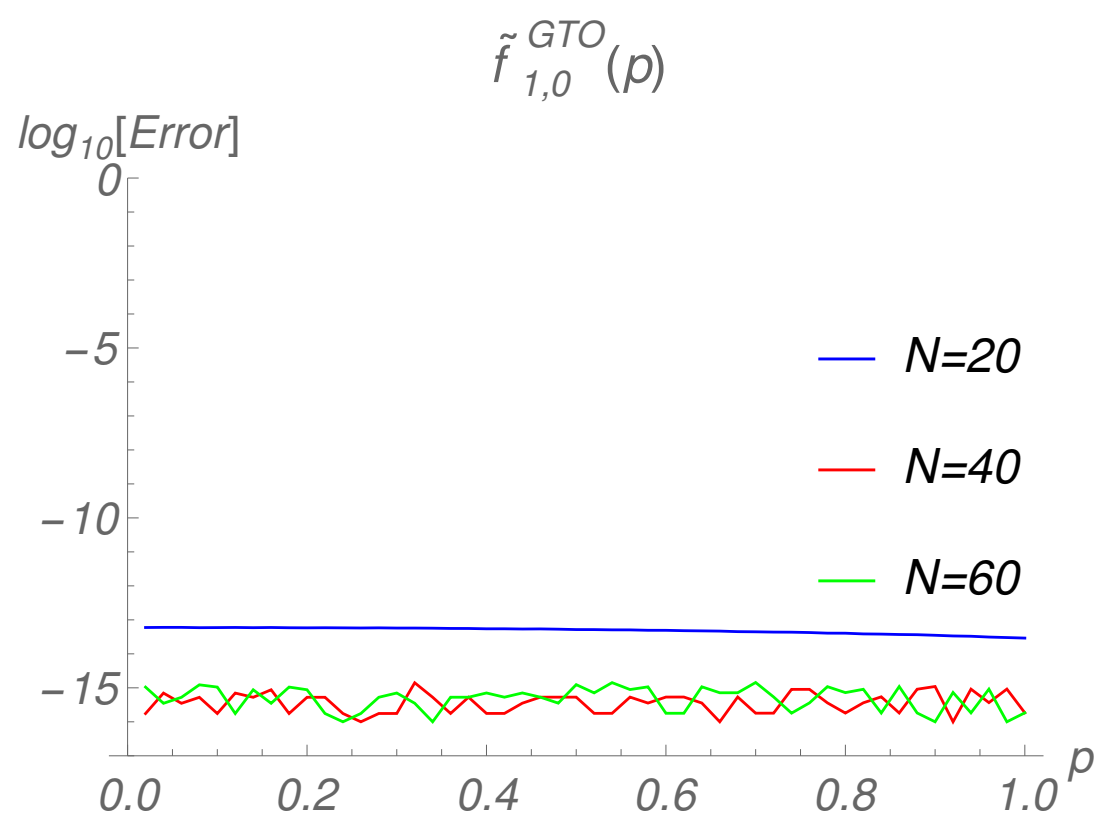

Figura 7.26: Gráfica del logaritmo del error relativo contra el valor de $p$ para la función tipo Gaussiana con $l=0$ y $\zeta=5.0$ en la región de $0.0-1.0$ en $p$. 


\subsubsection{2 $\quad l=1$}

En la Fig. (7.27) se muestra el error como una función de $p$ para cuadraturas de orden $N=20$ y $N=40$ basadas en las funciones de peso $e^{-r} \Theta_{0}(r)$ y $e^{-r} \Theta_{1}(r)$. Las gráficas del cálculo de $\widetilde{f}_{2,1}^{G T O}(p)$ muestran que el método funciona mejor a medida que $p$ disminuye para un orden de cuadratura particular. Al igual que con $l=0$ no se consiguen buenos resultados para $p$ grandes lo cual inidica que el método no es asintótico.

El método funciona mejor en regiones de $p$ pequeñas. Además la cuadratura de cuarenta puntos produce resultados esencialmente exactos para $p<15$.

En la Fig. (7.28) se presenta el análisis del error con diferentes órdenes de cuadratura para $0<p \leq 1$. Observamos que con los órdenes de cuadratura presentados se consiguen resultados razonables. Cuando se incrementa el orden de la cuadratura mejora el funcionamiento del método, sin embargo, el método comienza a perder precisión en la región cercana a cero.

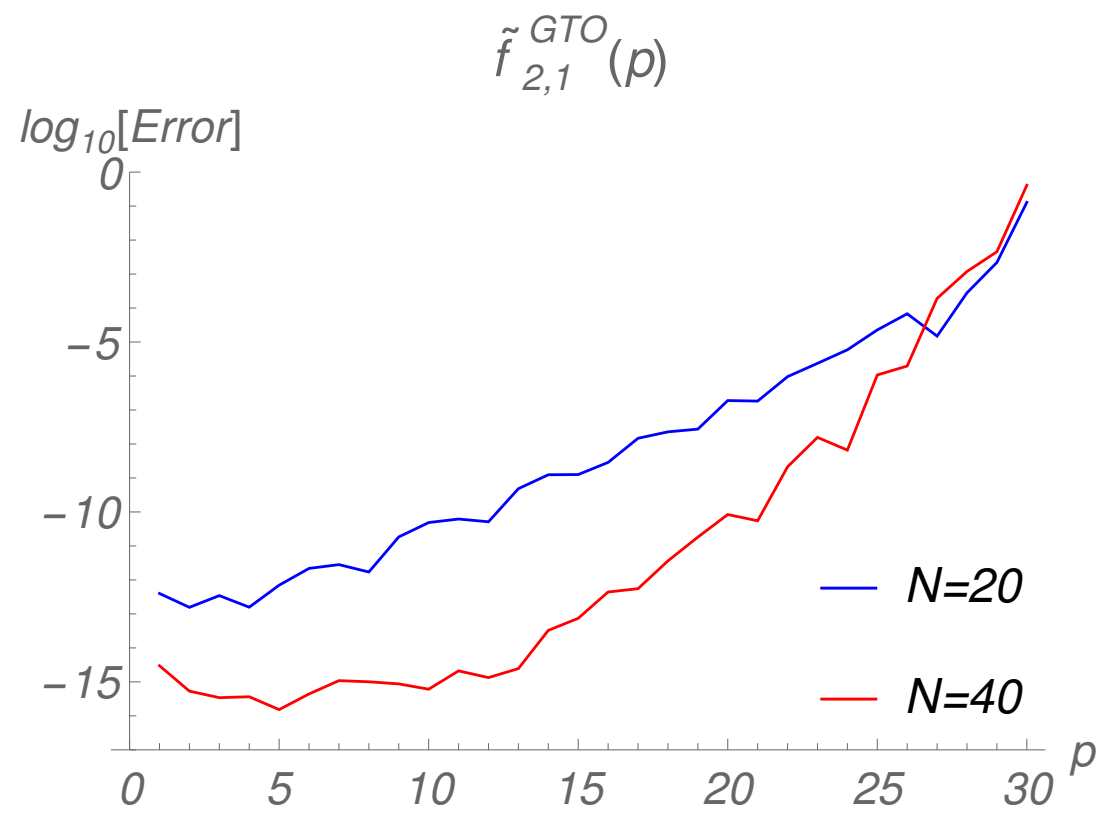

Figura 7.27: Gráfica del logaritmo del error relativo contra el valor de $p$ para la función tipo Gaussiana con $l=1$ y $\zeta=5.0$ en la región de $1.0-30.0$ en $p$. 




Figura 7.28: Gráfica del logaritmo del error relativo contra el valor de $p$ para la función tipo Gaussiana con $l=1$ y $\zeta=5.0$ en la región de $0.0-1.0$ en $p$.

\subsubsection{3 $l=2,3$}

En la Fig. (7.29) se presenta el error como una función de $p$ para cuadraturas de orden $N=20$ y $N=40$ basadas en las funciones de peso $e^{-r} \Theta_{0}(r)$, $e^{-r}(r+2)$ y $e^{-r} \Theta_{2}(r)$. Las gráficas del cálculo de $\widetilde{f}_{3,2}^{G T O}(p)$ muestran que el método funciona mejor a medida que $p$ disminuye para un orden de cuadratura particular. Al igual que con $l=0$ y $l=1$ no se consiguen buenos resultados para $p$ grandes.

En este caso el método no funciona para regiones de $p$ pequeñas además comienza a fallar para valores cercanos a $p=1$. La cuadratura de cuarenta puntos produce resultados esencialmente exactos para $2<p<15$.

En la Fig. (7.30) mostramos las gráficas para el cálculo de la función $\widetilde{f}_{4,3}^{G T O}(p)$ con $l=3$ que tiene un comportamiento similar al caso anterior. Además de que se acentúa el problema de la precisión en la región cercana a cero. 




Figura 7.29: Gráfica del logaritmo del error relativo contra el valor de $p$ para la función tipo Gaussiana con $l=2$ y $\zeta=5.0$ en la región de $1.0-31.0$ en $p$.



Figura 7.30: Gráfica del logaritmo del error relativo contra el valor de $p$ para la función tipo Gaussiana con $l=3$ y $\zeta=5.0$ en la región de $1.0-32.0$ en $p$. 


\subsubsection{Gaussiana sin transformar}

El método funciona bien para expresiones de tipo Gaussiano en las que no se aplica la transformada de Bessel esférica en la región asintótica $(p \geq 10)$. Es decir en integrales donde el término $r^{2}$ no aparece y la función de peso es $w(r)=e^{-r} \Theta_{0}(r)$. Para este caso tenemos que al sustituir en la ecuación (7.1.22) el valor de la integral $I(p)$ es diferente de cero.

El problema en la aplicación de la metodología a las funciones de base Gaussianas es el elemento Jacobiano de integración en la transformada de Bessel esférica. Sin embargo el método puede aplicarse a una integral con función general de Bessel esférica. La Fig. (7.31) muestra los resultados para los cálculos de $\int_{0}^{\infty} f(r) j_{0}(p r) \mathrm{d} r$ con $f(r)=e^{-r^{2}}$. Observamos que el método funciona bien en las regiones de $p$ más grandes y se pueden conseguir resultados más precisos para cuadraturas de orden superior. En este caso no es posible obtener resultados significativos para valores de $p$ cercanos a cero. Existen regiones de $p$ donde la cuadratura de orden inferior produce mejores resultados que la de orden superior y problemas con la representación de la máquina para las cuadraturas de orden superior.

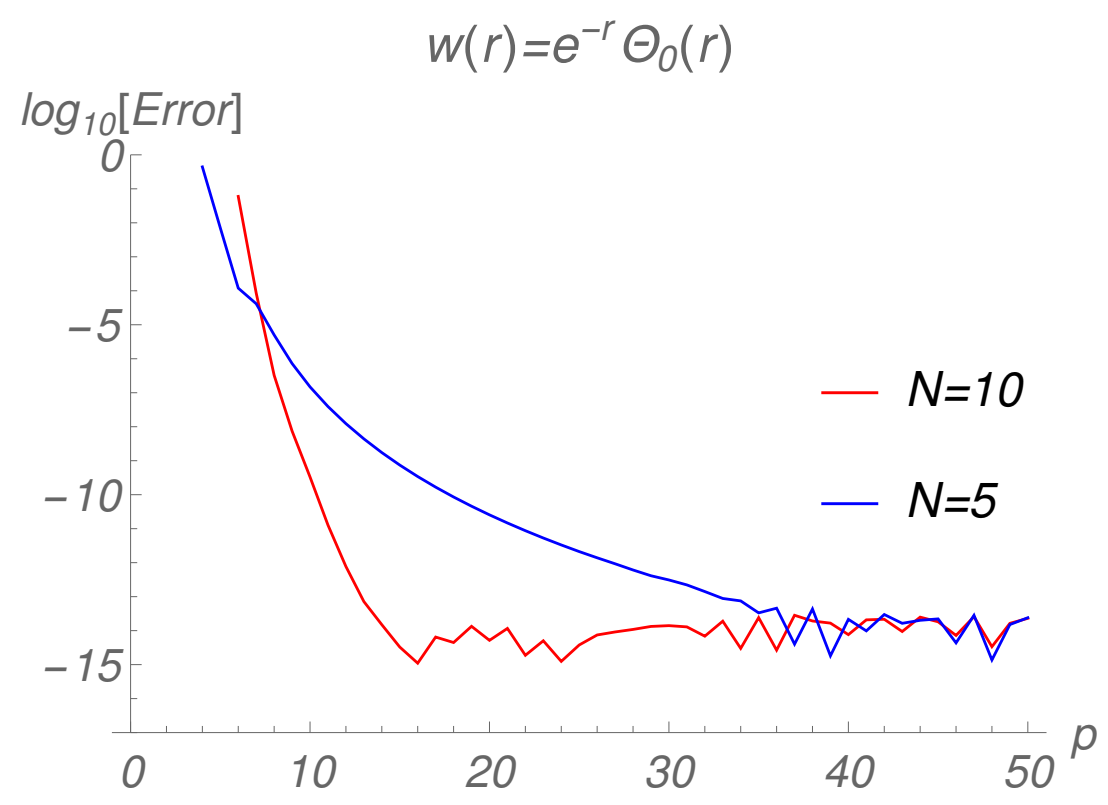

Figura 7.31: Gráfica del logaritmo del error relativo contra el valor de $p$ para la función Gaussiana sin transformar $f(r)=e^{-r^{2}}$ utilizando la función de peso $w(r)=e^{-r} \Theta_{0}(r)$ en la región de $1.0-50.0$ en $p$. 


\subsubsection{Aplicación a sistemas atómicos: extensión a orbitales}

Para esta aplicación se utilizó la nueva metodología para funciones de base tipo Slater (7.1.1). La ventaja en este caso es que se toma a la expansión del orbital $\phi_{i}(n, l)$ para obtener la transformada de Bessel esférica correspondiente a la misma simetría, es decir, con el mismo valor de $l$. Por lo cual ya no se calcula cada función de base tipo Slater $\chi_{i, l}$ de manera individual para después sumarse. Estudiamos el comportamiento del método en la región $1 \leq p \leq 50$.

\subsubsection{1 Átomo de Helio $(H e)$}

Es importante señalar que para el orbital atómico $\phi(1 s)$ se calculan las funciones de base tipo Slater $l=0$ relacionadas con la función de peso $w(r)=e^{-r} \Theta_{0}(r)$.

La Fig. (7.32) presenta las gráficas para el orbital atómico $\phi(1 s)$ donde la cuadratura de veinte puntos produce valores exactos para $p>10$. Las cuadraturas con $N=40,60$ producen resultados exactos para $p>5$.

Una observación importante es que se requieren de órdenes de cuadratura mayores que cuando se calculan funciones de base tipo Slater de manera individual. Además no se consiguen resultados en la región $0<p \leq 1$.



Figura 7.32: Gráfica del logaritmo del error relativo contra el valor de $p$ para el orbital $\phi(1 s)$ para el átomo de Helio región de 1.0 - 50.0 en $p$. 


\subsubsection{2 Átomo de Neon $(\mathrm{Ne})$}

En este caso para los orbitales atómicos $\phi(1 s)$ y $\phi(2 s)$ también se calculan las funciones de base tipo Slater $l=0$ relacionadas con la función de peso $w(r)=e^{-r} \Theta_{0}(r)$.

En las Figs. (7.33 - 7.34) presentamos las gráficas para los orbitales atómicos $\phi(1 s)$ y $\phi(2 s)$ respectivamente donde la cuadratura de sesenta puntos produce valores exactos para $p \approx 15$ en ambos casos. Mientras que se requieren de cuadraturas de orden mayor con $N=135$ para producir resultados exactos en $p=10$.

Tampoco es posible conseguir resultados satisfactorios en la región $0<$ $p \leq 1$. Inclusive se aprecia que el comportamiento para $\phi(2 s)$ en la región cercana a cero no es monótono decreciente como en $\phi(1 s)$.

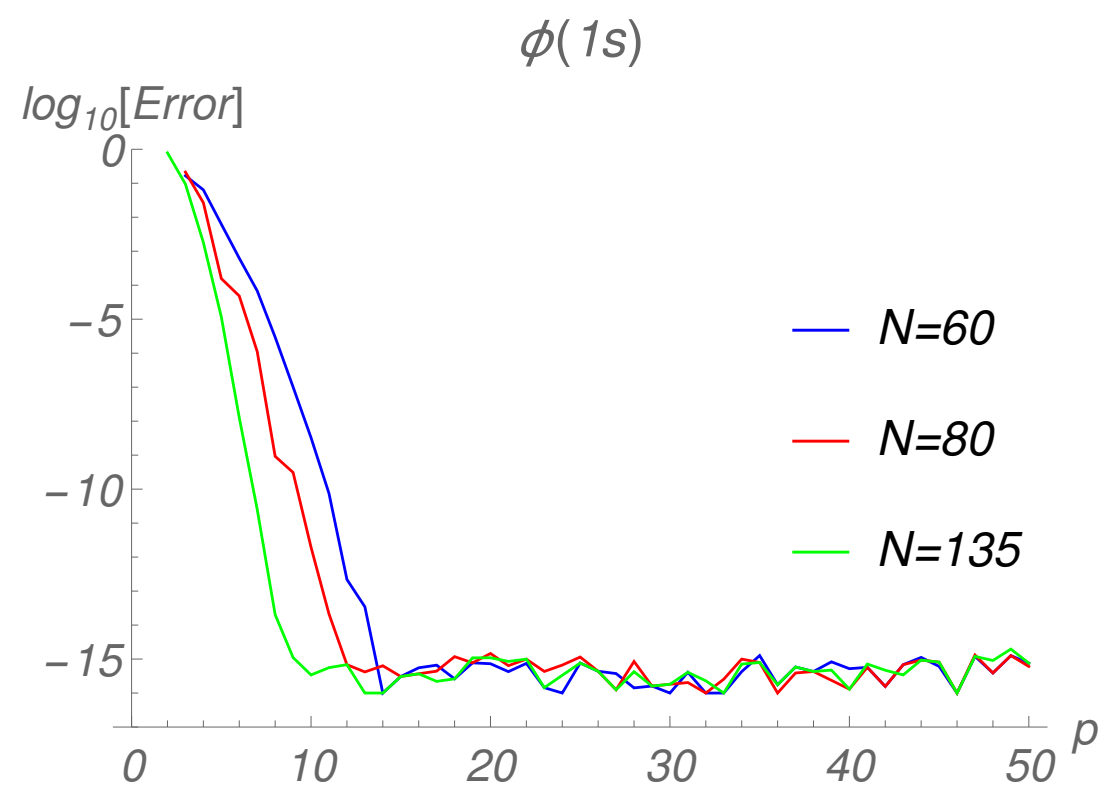

Figura 7.33: Gráfica del logaritmo del error relativo contra el valor de $p$ para el orbital $\phi(1 s)$ para el átomo de Neón en la región de 1.0 - 50.0 en $p$. 


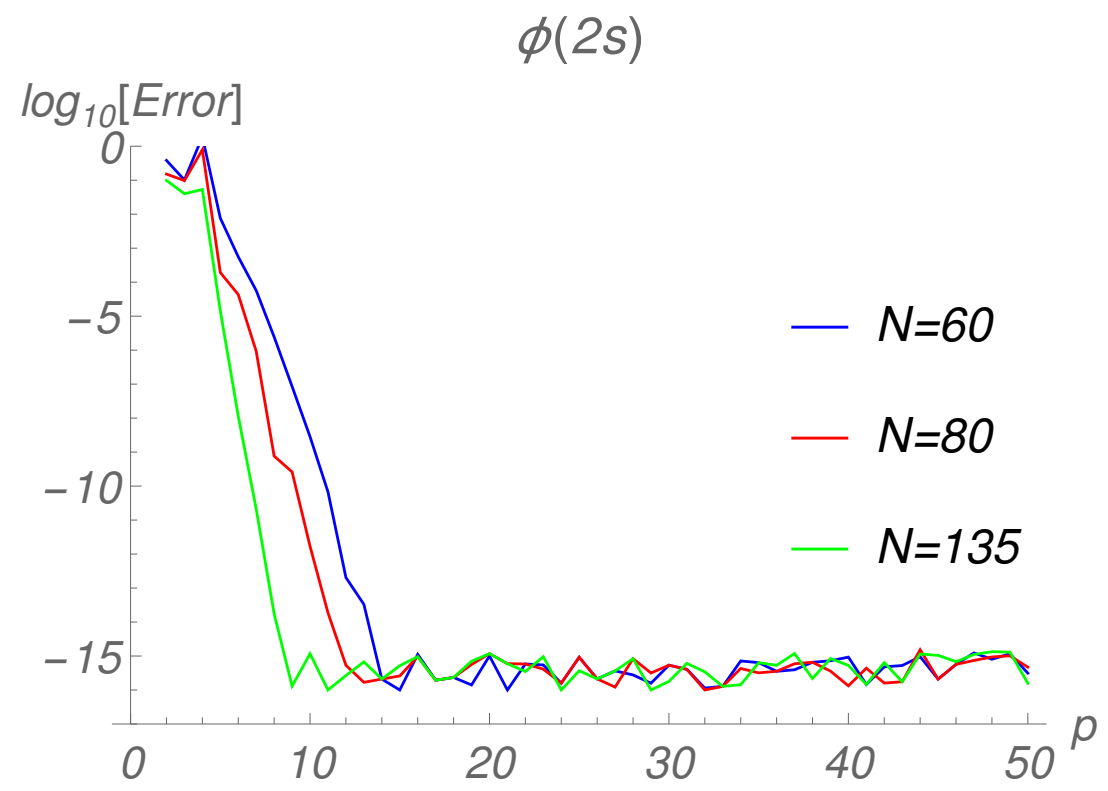

Figura 7.34: Gráfica del logaritmo del error relativo contra el valor de $p$ para el orbital $\phi(2 s)$ para el átomo de Neón en la región de 1.0 - 50.0 en $p$.

La Fig. (7.35) presenta las gráficas para el orbital atómico $\phi(2 p)$ donde se calculan las funciones de base tipo Slater $l=1$ relacionadas con la función de peso $w(r)=e^{-r} \Theta_{1}(r)$.

La cuadratura de sesenta puntos genera valores exactos para $p>15$. Además las cuadraturas con $N=80,135$ producen resultados exactos para $p>10$. El método falla en $0<p \leq 1$. 


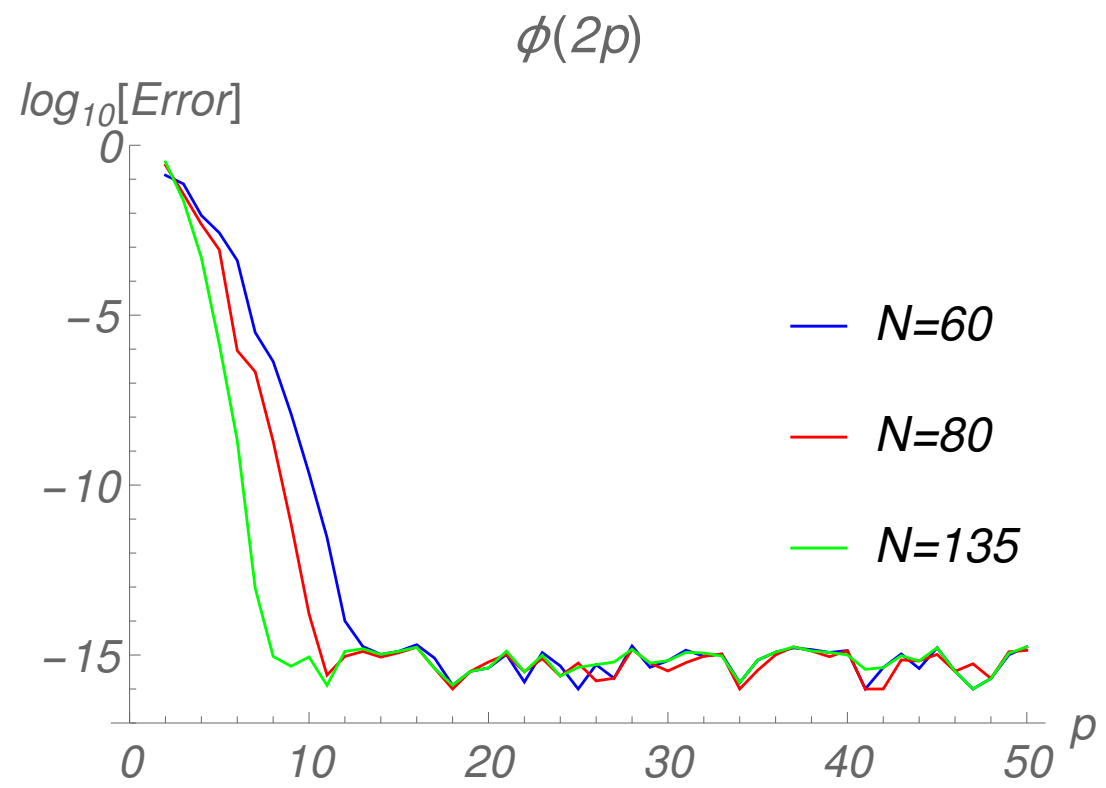

Figura 7.35: Gráfica del logaritmo del error relativo contra el valor de $p$ para el orbital $\phi(2 p)$ para el átomo de Neón en la región de 1.0 - 50.0 en $p$.

Se probaron dos valores diferentes de $\zeta$ correspondientes a dos funciones de base tipo Slater las que aparecen en el orbital $\phi(1 s)$ para el átomo de Neón. Para la función de base con el primer valor $\zeta=1.304155$ en la Fig. (7.36) observamos que el mejor funcionamiento del método es para $p$ grandes donde se amortigua la oscilación. Sin embargo para la segunda función con valor $\zeta=16.354484$ en la Fig. (7.37) vemos que el comportamiento oscilatorio del integrando no se amortigua lo suficiente a medida que $p$ aumenta. En este caso las oscilaciones son más fuertes para la región de $p$ pequeñas. Por lo que al incrementar el valor de $\zeta$ la metodología presenta dificultades en regiones de $p$ pequeñas. Esto demuestra la influencia del coeficiente en el exponente sobre el comportamiento del método. 


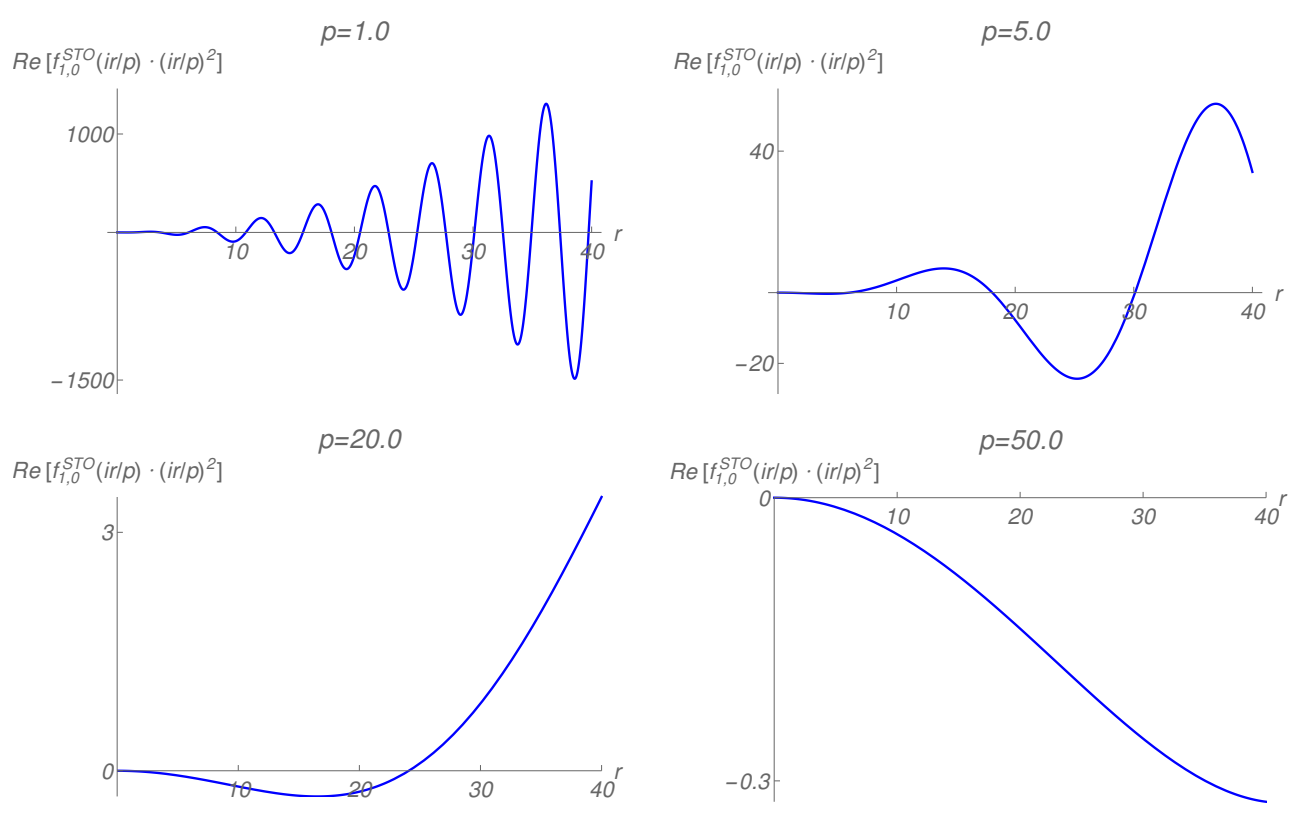

Figura 7.36: Gráfica de $\operatorname{Re}\left[f_{1,0}^{S T O}(\imath r / p) \cdot(\imath r / p)^{2}\right]$ contra $r$ para diferentes valores de $p$ y $\zeta=1.304155$.

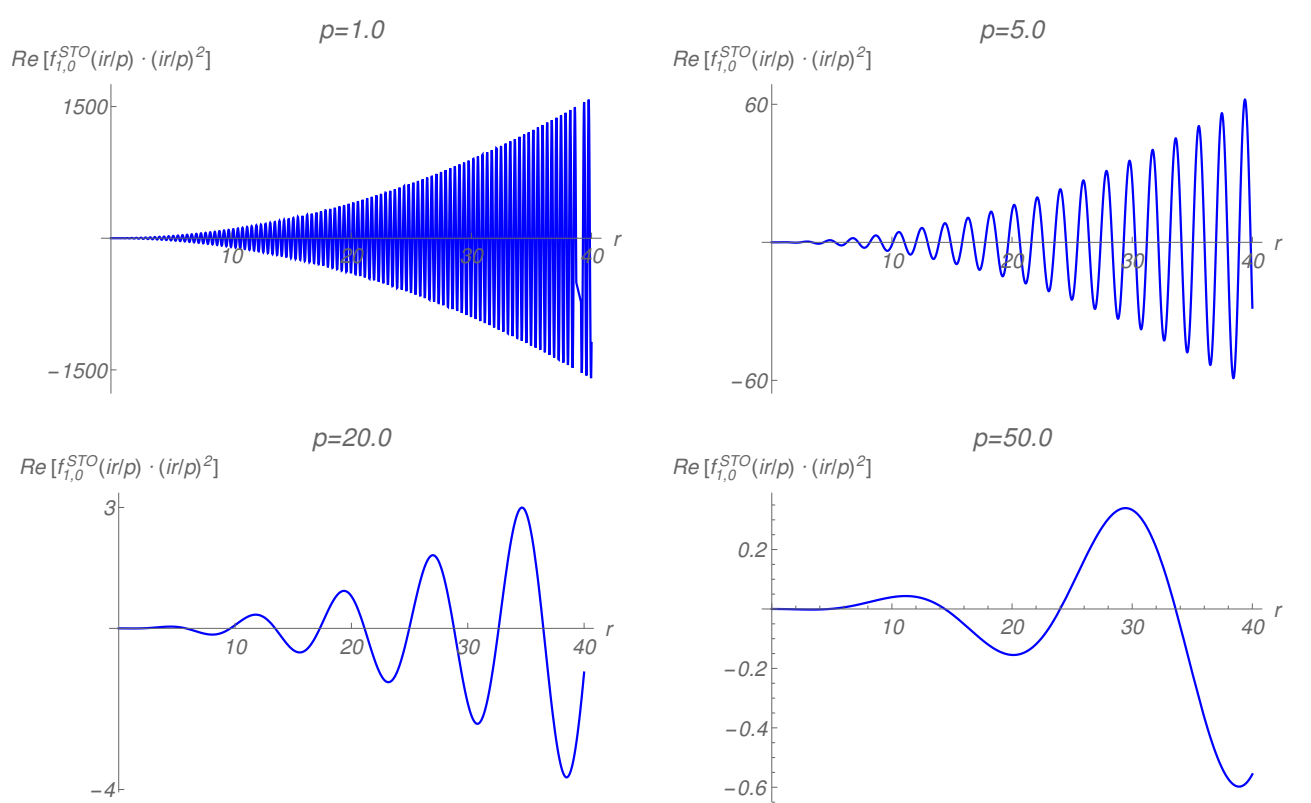

Figura 7.37: Gráfica de $\operatorname{Re}\left[f_{1,0}^{S T O}(\imath r / p) \cdot(\imath r / p)^{2}\right]$ contra $r$ para diferentes valores de $p$ y $\zeta=16.354484$. 


\subsubsection{3 Átomo de Hierro $(\mathrm{Fe})$}

La Fig. (7.38) presenta las gráficas para el orbital atómico $\phi(3 d)$ donde se calculan las funciones de base tipo Slater $l=2$ relacionadas con la función de peso $w(r)=e^{-r} \Theta_{2}(r)$.

Observamos que la cuadratura de sesenta puntos da valores exactos para $p=15$. Además la cuadratura con $N=135$ produce resultados exactos para $p>8$. También el método falla en $0<p \leq 1$.

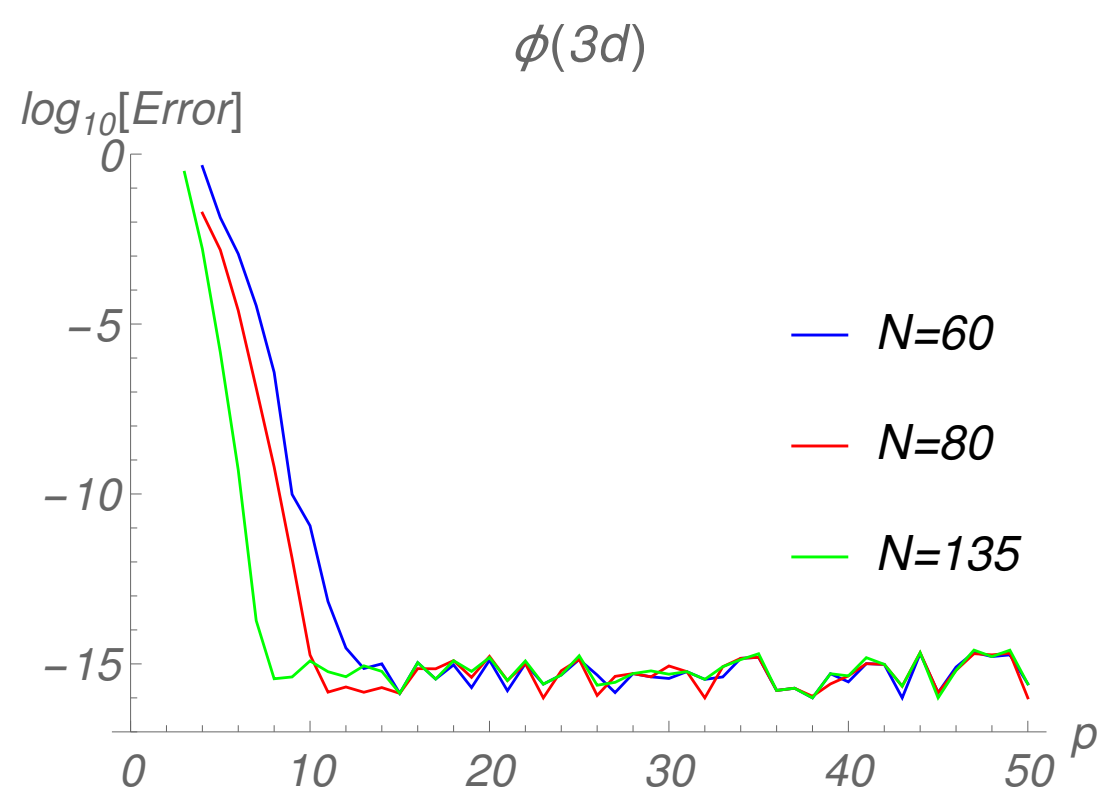

Figura 7.38: Gráfica del logaritmo del error relativo contra el valor de $p$ para el orbital $\phi(3 d)$ para el átomo de Hierro en la región de $1.0-50.0$ en $p$.

Finalmente debemos mencionar que no existe una dependencia marcada con respecto al valor de $l$ y el funcionamiento adecuado de la nueva metodología. Lo anterior significa que es más importante la contribución de los coeficientes en el exponente $\zeta$ ya que valores grandes de estos provocan que el método falle para valores de $p$ pequeños. Por otro lado valores de $\zeta$ cercanos a uno o menores hacen que el método funcione bien en la región asintótica y no asintótica. 


\subsection{Extensión de la metodología para las funciones de base tipo Slater usando el parámetro $\alpha$}

Es posible implementar el procedimiento usado para las funciones de base tipo Gaussianas a las funciones de base tipo Slater con el propósito de obtener mejores resultados en la región no asintótica de $p$. Es decir, podemos introducir el parámetro $\alpha$ (ver ecuaciones 7.2.24-7.2.26) y con esto usar las mismas funciones de peso que en la base tipo Gaussiana, ver la Tabla (7.5). Lo anterior significa que intercambiamos la función a evaluar por las funciones de base tipo Slater ya que con el parámetro se mejora el comportamiento del método al incrementar el valor de $\zeta$ en las funciones de base tipo Slater.

A continuación se muestra el comparativo de una función de base tipo Slater $\chi_{7}=N_{7} e^{-\zeta r}$ para el orbital $\phi(1 s)$ del Neón que tiene un coeficiente $\zeta=16.354484$. Observamos que sin usar el parámetro $(\alpha=0)$ y utilizando un valor de $\alpha=20.0$ para los mismos órdenes de cuadratura la introducción del parámetro $\alpha$ permite conseguir mejores resultados incluso con $N=10$.


Figura 7.39: Gráfica del logaritmo del error relativo contra el valor de $p$ para la función tipo Slater $\chi_{7} \operatorname{con} \zeta=16.354484$ para el átomo de Neón con dos valores del parámetro $\alpha$.

En la Fig. (7.40) presentamos una función de base tipo Slater $\chi_{7}=N_{7} e^{-\zeta r}$ para el orbital $\phi(2 p)$ del Neón con un coeficiente $\zeta=25.731219$. Se muestra un comportamiento similar al de la figura anterior (7.39) ya que el parámetro $\alpha=20.0$ permite conseguir valores de $p$ en la región no asintótica con un orden de cuadratura de $N=10$. 

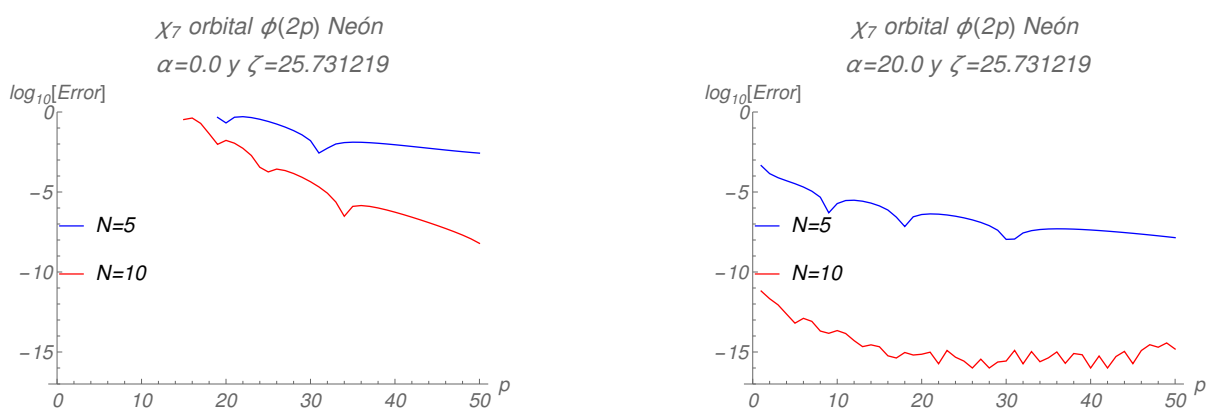

Figura 7.40: Gráfica del logaritmo del error relativo contra el valor de $p$ para la función tipo Slater $\chi_{7} \operatorname{con} \zeta=25.731219$ para el átomo de Neón con dos valores del parámetro $\alpha$.

Ahora veamos una prueba más fuerte ya que calculamos un orbital completo en el que se involucran varias funciones de base tipo Slater que poseen la misma geometría (igual valor de $l$ ). Esto tiene la ventaja de calcular todo en un sólo paso en vez de hacerlo individualmente para cada función de base tipo Slater.

En la Fig. (7.41) se muestra el comparativo para el cálculo del orbital atómico $\phi(1 s)$ del átomo de Neón usando dos valores de $\alpha$ distintos. En este caso la gráfica de la izquierda no tiene parámetro $\alpha$ mientras que la gráfica de la derecha tiene un parámetro $\alpha>0$. Observamos que para órdenes de cuadratura más bajos es posible conseguir mejores resultados usando el parámetro $\alpha=13.0$. Incluso es posible obtener valores de $p$ en la región no asintótica.
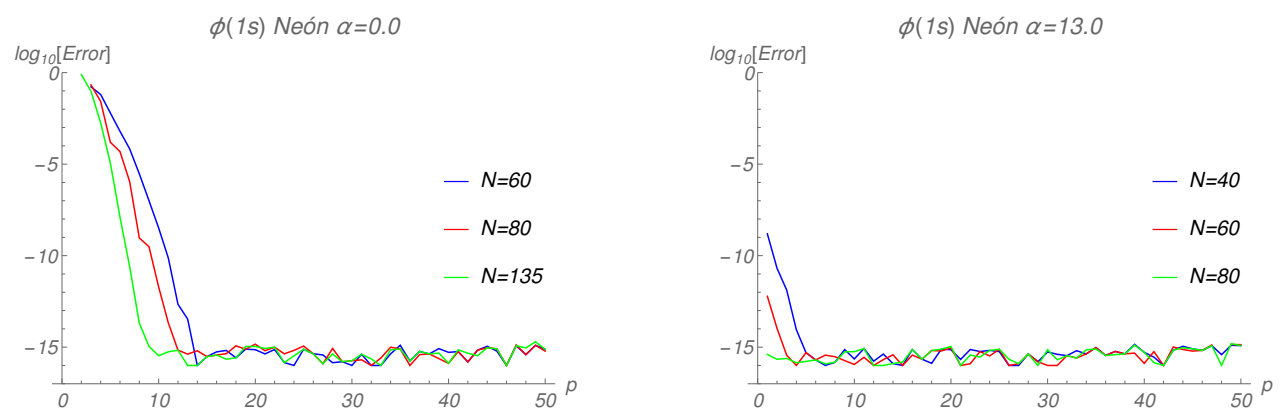

Figura 7.41: Gráfica del logaritmo del error relativo contra el valor de $p$ para el orbital $\phi(1 s)$ para el átomo de Neón en la región de $1.0-50.0$ en $p$ con dos valores del parámetro $\alpha$. 
Algo muy similar ocurre para el cálculo del orbital $\phi(2 p)$ en el mismo átomo de Neón ver Fig. (7.42) donde es notable el orden de cuadratura necesario $(N=80)$ para alcanzar buenos resultados en las regiones asintóticas y no asintóticas de $p$.


Figura 7.42: Gráfica del logaritmo del error relativo contra el valor de $p$ para el orbital $\phi(2 p)$ para el átomo de Neón en la región de $1.0-50.0$ en $p$ con dos valores del parámetro $\alpha$.

\subsection{Comparación con otra metodología}

A continuación se compara el método con otra metodología que se desarrolla en [64] como una alternativa para resolver el problema de calcular numéricamente la transformada de Bessel esférica para funciones de base tipo Slater y Gaussianas.

Este procedimiento numérico calcula la transformada de Bessel esférica a través de la transformada de Fourier rápida con una fórmula de recurrencia. El método utiliza una representación integral de la función de Bessel esférica considerando un cambio de variable en la integración. En [64] grafican el error absoluto el cual definen como Error $=\log _{10}\left|\widetilde{f}_{\text {num }}(k)-\widetilde{f}_{\text {ana }}(k)\right|$ contra la variable momento la cual llaman $k$ y está dada en unidades atómicas inversas. Además presentan a las distintas curvas en las gráficas en términos de mallas $N$.

\subsubsection{Funciones de base tipo Slater}

A partir de los resultados mostrados en la sección (7.4.1.1) tenemos que para una función de base tipo Slater con $l=0$ el método que proponemos requiere de órdenes de cuadratura bajos $N=5,10$. Asimismo nuestro método es competitivo ya que se consiguen mejores resultados que los mostrados en el 
método de Toyoda, ver Fig. (7.43) inciso (a), aun cuando ellos grafican contra el error absoluto y nosotros lo hacemos contra el error relativo. Observamos en el inciso (a) de la Fig. (7.43) que el método no es asintótico y con los órdenes de malla $N=256,2048$ no alcanzan el valor analítico en $l=0$. En términos generales la metodología que proponemos es convergente asintóticamente e inclusive funciona para regiones donde $p<1$ ya que en [64] no presentan un estudio de la región no asintótica.

También de acuerdo a los resultados mostrados en la sección (7.4.1.1) podemos inferir que la prueba para $l=15$ que presenta Toyoda en el inciso (b) de la Fig. (7.43) con nuestra metodología se alcanzaría la convergencia en $p<2$ usando órdenes de cuadratura bajos.
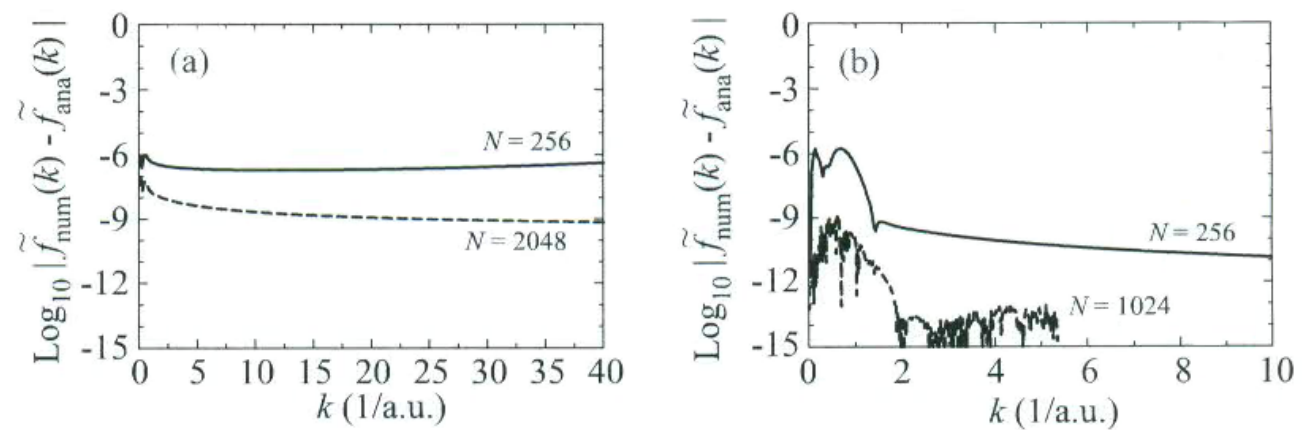

Figura 7.43: Gráfica del logaritmo del error absoluto contra el valor de $k$ para diferentes mallas $N$. (a) Para $l=0$. (b) Para $l=15$. Figura tomada de [64].

\subsubsection{Funciones de base tipo Gaussianas}

En este caso tenemos que nuestra metodología presenta un buen funcionamiento ver Figs. (7.44 - 7.45) para una región de $0<p \leq 10$ donde los resultados obtenidos superan a los mostrados en la Fig. (7.46) para un orden de cuadratura de cuarenta puntos. La Fig. (7.46) muestra que el método de Toyoda no es asintótico y para ningún valor de malla $N$ se alcanza el valor analítico. Es importante mencionar que nuestra metodología falla para valores de $p$ grandes ver la sección (7.4.2) por lo que ambos métodos no son asintóticos. Algo interesante es el hecho de que este tipo de transformaciones con funciones de base Gaussianas muestran un comportamiento problemático en ambos métodos para alcanzar buenos resultados con valores de $p$ grandes. 


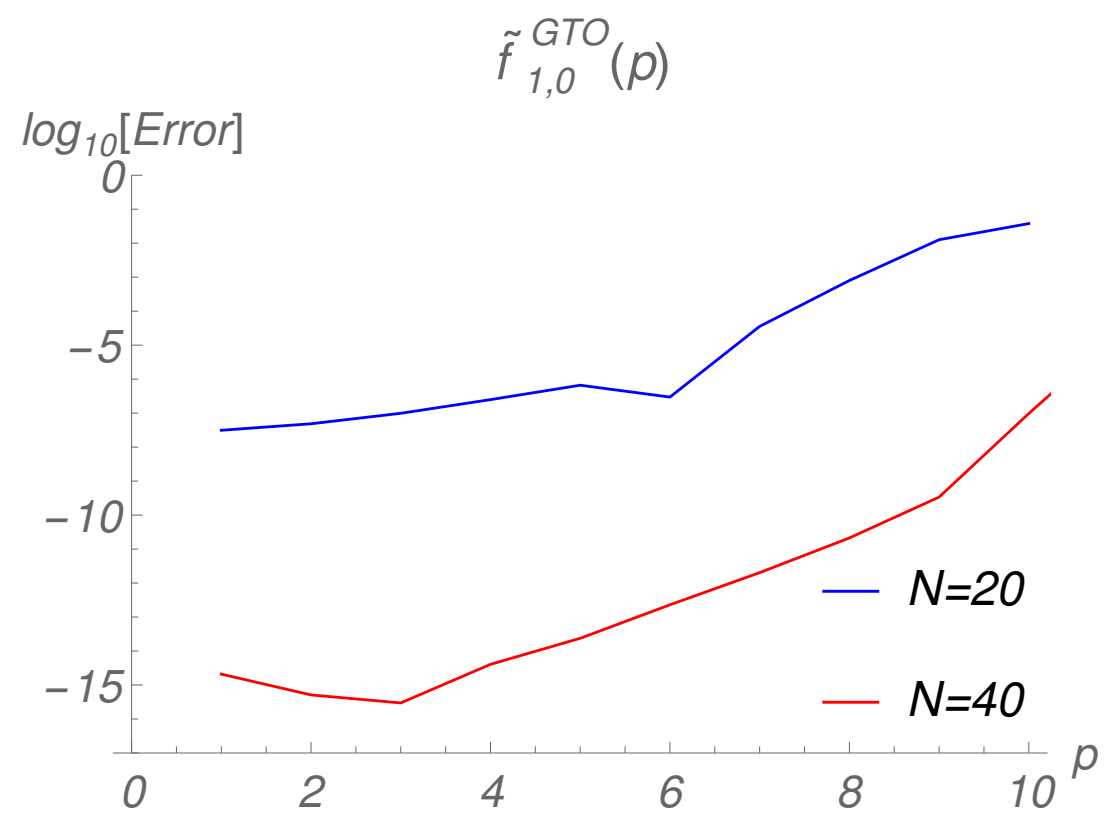

Figura 7.44: Gráfica del logaritmo del error relativo contra el valor de $p$ para la función tipo Gaussiana con $l=0$ y $\zeta=1.0$ en la región de $1.0-10.0$ en $p$.

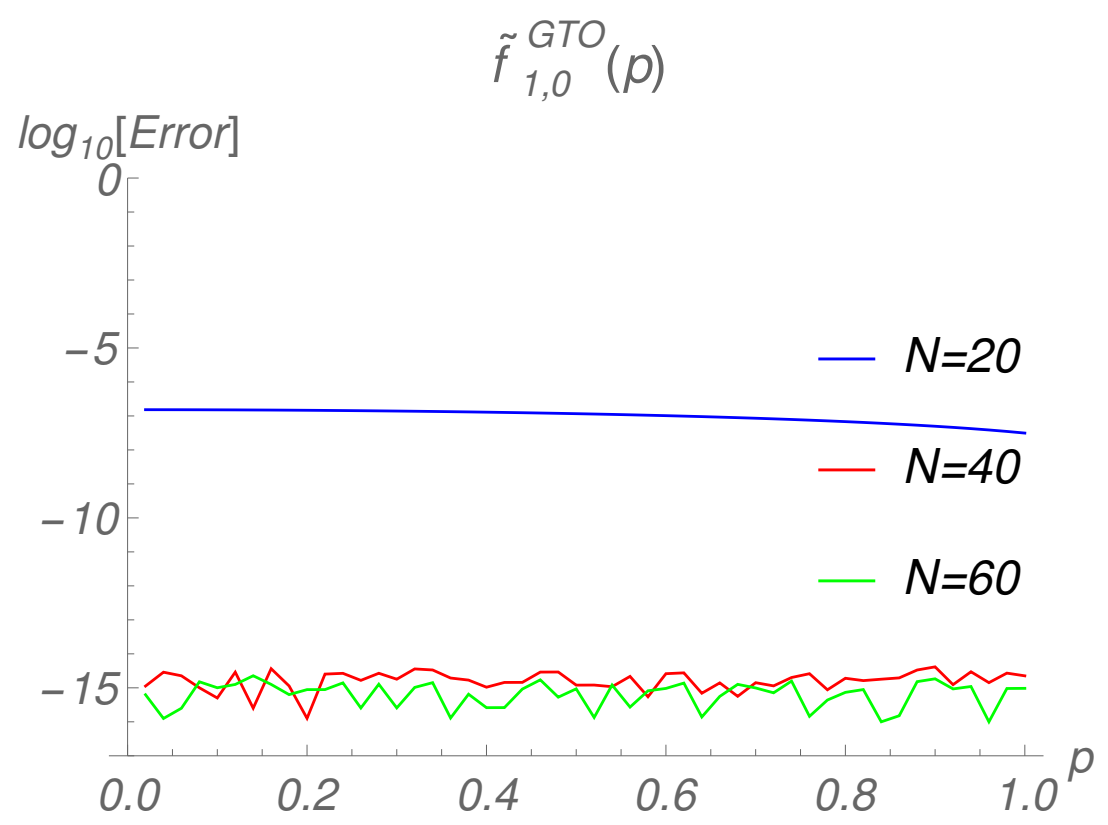

Figura 7.45: Gráfica del logaritmo del error relativo contra el valor de $p$ para la función tipo Gaussiana con $l=0$ y $\zeta=1.0$ en la región de $0.0-1.0$ en $p$. 




Figura 7.46: Gráfica del logaritmo del error absoluto contra el valor de $k$ para $l=0$ se presentan diferentes mallas con valores de $N=128,256,512,1024$ donde las curvas correspondientes a cada valor de $N$ vienen en orden descendente. Figura tomada de [64].

\subsection{Conclusiones}

Una nueva metodología fue desarrollada para aplicarla en el cálculo numérico de integrales que involucran funciones de Bessel esférica $j_{0}(r), j_{1}(r), j_{2}(r)$, $j_{3}(r)$ y $j_{4}(r)$. Por lo que se definieron cuadraturas Gaussianas basadas en funciones de peso relacionadas con los polinomios de Bessel inversos usadas en la transformada de Bessel esférica para funciones con bases tipo Slater y Gaussiana.

Los resultados numéricos muestran que el método funciona muy bien en las regiones asintóticas con $p$ grande incluso con cuadraturas de orden bajo. Los resultados exactos para $p$ pequeñas se pueden obtener mediante el incremento del orden de la cuadratura así los valores están convergiendo monótonamente al resultado analítico. Sin embargo no es posible obtener valores para $p<0.2$. También se debe resaltar que este método es competitivo al compararse con otra metodología [64]. 
El método se puede aplicar para funciones de tipo Gaussiano donde la introducción de un parámetro evita que falle como en el caso del oscilador armónico en dos dimensiones. Además este parámetro provoca que el método ya no sea de tipo asintótico. Los valores óptimos del parámetro se encuentran acotados en cierto intervalo $(\alpha \approx[20,30])$. Para las funciones Gaussianas con valores de $p$ grandes el método falla. Aunque presenta dificultades este método es competitivo para ciertas regiones al compararse con otra metodología [64].

Se aplicó la metodología para transformar orbitales atómicos al espacio de momentos obteniéndose buenos resultados en la región asintótica de $p$. Mostramos que con valores de $\zeta$ grandes para funciones de base tipo Slater el método pierde precisión en valores de $p$ pequeños. Por otro lado se puede mejorar el método en esta región no asintótica introduciendo el parámetro $\alpha$ como en el caso de las funciones de base tipo Gaussianas. 


\section{Conclusiones generales}

- Los resultados numéricos muestran que en general la metodología funciona muy bien en regiones de $p$ asintóticas para cuadraturas de orden bajo $N=10$ en los casos de la transformada de Hankel del átomo de hidrógeno y la transformada de Bessel esférica para funciones de base tipo Slater.

- En la región no asintótica es difícil obtener una mayor precisión incrementando el orden de la cuadratura para $p<0.2$. La metodología es convergente en esta región para la transformada de Hankel del átomo de hidrógeno y para la transformada de Bessel esférica en funciones de base tipo Slater.

- El método de Wong no funciona para la transformación al espacio de momentos en el caso del oscilador armónico en dos dimensiones. Sin embargo es posible utilizar la metodología para calcular integrales con funciones Gaussianas asociadas a funciones de Bessel entero en las que no aparece la transformada de Hankel.

- La metodología nueva no funciona para la transformación al espacio de momentos en el caso de las funciones de base tipo Gaussianas en tres dimensiones. Sin embargo la introducción de un parámetro $\alpha$ permite obtener valores numéricos usando la metodología. Los resultados exactos para $p$ grandes no se pueden conseguir mediante el incremento del orden de la cuadratura ni modificando el valor del parámetro $\alpha$. Los resultados numéricos muestran que la metodología nueva funciona muy bien en la región no asintótica de $p$ incluso con cuadraturas de orden bajo. Además es posible utilizar la metodología para calcular integrales con funciones Gaussianas asociadas a funciones de Bessel esféricas en las que no aparece la transformada de Bessel esférica.

- La metodología para el caso de dos dimensiones es competitiva en la región asintótica al compararse con otro método [35] para funciones con comportamiento exponencial y Gaussiano.

- La metodología nueva para el caso de tres dimensiones es competitiva en la región asintótica y no asintótica al compararse con otro método [64] para funciones de base tipo Slater. Para el caso de las funciones de base tipo Gaussianas la metodología nueva resulta competitiva al compararse con respecto a [64] en la región no asintótica. En ambos casos nuestra metodología nueva requiere de órdenes de cuadratura menores a sesenta. 
- La metodología nueva fue aplicada para transformar orbitales atómicos al espacio de momentos dando buenos resultados en la región asintótica y mostrando la dependencia con respecto a los valores de los coeficientes en el exponente de las funciones de base tipo Slater. Además se muestra que los resultados en la región no asintótica pueden mejorarse introduciendo el parámetro $\alpha$. 


\section{Perspectivas}

- Aplicar esta metodología para la evaluación de integrales de repulsión electrón-electrón [72-74].

- Generar los polinomios ortogonales correspondientes y usar este método para calcular las integrales con intervalo de integración acotado por los límites $[a, b][75,76]$.

- Probar la metodología en espacios de dimensiones mayores $n>3$ para explorar su alcance.

- Examinar y desarrollar otras metodologías incluyendo las del plano complejo [77]. 


\section{Apéndice A}

\subsection{Coeficientes de recurrencia y cuadraturas Gaussianas para funciones de peso $w(x)=x^{m} K_{m}(x)$ para $m=0, m=1, m=2$ y $m=3$.}

\begin{tabular}{cc}
$\alpha_{n}$ & $\beta_{n}$ \\
\hline $0.636619772367581+00$ & $0.157079632679490+01$ \\
$0.257476461293110+01$ & $0.594715265430649+00$ \\
$0.455674548258986+01$ & $0.313882578680290+01$ \\
$0.654745603869502+01$ & $0.767060044233757+01$ \\
$0.854158085501622+01$ & $0.141964862164733+02$ \\
$0.105374441563584+02$ & $0.227188108611067+02$ \\
$0.125343315227843+02$ & $0.332387014752612+02$ \\
$0.145318810974393+02$ & $0.457567990542895+02$ \\
$0.165298876757858+02$ & $0.602735074118681+02$ \\
$0.185282251677061+02$ & $0.767890996444386+02$ \\
$0.205268112978841+02$ & $0.953037703243276+02$ \\
$0.225255898112020+02$ & $0.115817663720878+03$ \\
$0.245245207759544+02$ & $0.138330890231810+03$ \\
$0.265235749640011+02$ & $0.162843536521054+03$ \\
$0.285227304266007+02$ & $0.189355672072447+03$ \\
$0.305219703196624+02$ & $0.217867353592334+03$ \\
$0.325212814733259+02$ & $0.248378628063819+03$ \\
$0.345206534225286+02$ & $0.280889534924376+03$ \\
$0.365200777327563+02$ & $0.315400107655404+03$ \\
$0.385195475203576+02$ & $0.351910374966539+03$ \\
$0.405190571043985+02$ & $0.390420361694034+03$ \\
$0.425186017494634+02$ & $0.430930089493162+03$ \\
$0.445181774726063+02$ & $0.473439577379489+03$ \\
$0.465177808963684+02$ & $0.517948842157416+03$ \\
$0.485174091354139+02$ & $0.564457898763379+03$ \\
$0.505170597080629+02$ & $0.612966760543573+03$ \\
$0.525167304665085+02$ & $0.663475439480831+03$ \\
$0.545164195412281+02$ & $0.715983946381573+03$ \\
$0.565161252963000+02$ & $0.770492291031088+03$ \\
$0.585158462931839+02$ & $0.827000482323455+03$ \\
$0.605155812611341+02$ & $0.885508528370981+03$ \\
$0.625153290728580+02$ & $0.946016436596974+03$ \\
$0.645150887243528+02$ & $0.100852421381484+04$ \\
$0.665148593181015+02$ & $0.107303186629588+04$ \\
$0.685146400489843+02$ & $0.113953939982775+04$
\end{tabular}


-continuación-

\begin{tabular}{cc}
$\alpha_{n}$ & $\beta_{n}$ \\
\hline $0.705144301924028+02$ & $0.120804681976499+04$ \\
$0.725142290942177+02$ & $0.127855413107304+04$ \\
$0.745140361621808+02$ & $0.135106133836664+04$ \\
$0.765138508586072+02$ & $0.142556844594353+04$ \\
$0.785136726940801+02$ & $0.150207545781403+04$ \\
$0.805135012220208+02$ & $0.158058237772724+04$ \\
$0.825133360339874+02$ & $0.166108920919424+04$ \\
$0.845131767555882+02$ & $0.174359595550866+04$ \\
$0.865130230429185+02$ & $0.182810261976506+04$ \\
$0.885128745794420+02$ & $0.191460920487539+04$ \\
$0.905127310732539+02$ & $0.200311571358366+04$ \\
$0.925125922546707+02$ & $0.209362214847921+04$ \\
$0.945124578741022+02$ & $0.218612851200862+04$ \\
$0.965123277001677+02$ & $0.228063480648648+04$ \\
$0.985122015180230+02$ & $0.237714103410514+04$ \\
$0.100512079127872+03$ & $0.247564719694356+04$ \\
$0.102511960343637+03$ & $0.257615329697529+04$ \\
$0.104511844991776+03$ & $0.267865933607590+04$ \\
$0.106511732910210+03$ & $0.278316531602953+04$ \\
$0.108511623947375+03$ & $0.288967123853510+04$ \\
$0.110511517961358+03$ & $0.299817710521183+04$ \\
$0.112511414819121+03$ & $0.310868291760444+04$ \\
$0.114511314395801+03$ & $0.322118867718777+04$ \\
$0.116511216574074+03$ & $0.333569438537120+04$ \\
$0.118511121243582+03$ & $0.345220004350261+04$ \\
$0.120511028300409+03$ & $0.357070565287206+04$ \\
$0.122510937646605+03$ & $0.369121121471521+04$ \\
$0.124510849189755+03$ & $0.381371673021649+04$ \\
$0.126510762842580+03$ & $0.393822220051201+04$ \\
$0.128510678522579+03$ & $0.406472762669226+04$ \\
$0.130510596151696+03$ & $0.419323300980464+04$ \\
$0.132510515656019+03$ & $0.432373835085583+04$ \\
$0.134510436965497+03$ & $0.445624365081392+04$ \\
$0.136510360013691+03$ & $0.459074891061051+04$ \\
$0.138510284737532+03$ & $0.472725413114254+04$
\end{tabular}


-continuación-

\begin{tabular}{cc}
$\alpha_{n}$ & $\beta_{n}$ \\
\hline $0.140510211077108+03$ & $0.486575931327411+04$ \\
$0.142510138975463+03$ & $0.500626445783813+04$ \\
$0.144510068378411+03$ & $0.514876956563788+04$ \\
$0.146509999234367+03$ & $0.529327463744842+04$ \\
$0.148509931494187+03$ & $0.543977967401801+04$ \\
$0.150509865111023+03$ & $0.558828467606939+04$ \\
$0.152509800040185+03$ & $0.573878964430091+04$ \\
$0.154509736239018+03$ & $0.589129457938776+04$ \\
$0.156509673666780+03$ & $0.604579948198296+04$ \\
$0.158509612284537+03$ & $0.620230435271841+04$
\end{tabular}

Tabla 10.1: Coeficientes de recurrencia para la función de peso $w(x)=K_{0}(x)$.

\begin{tabular}{cc} 
Abscisas & Pesos \\
\hline $8.982513946507351-02$ & $6.829948961994860-01$ \\
$5.897923377431657-01$ & $5.830426341981469-01$ \\
$1.574481470283905+00$ & $2.403056562639477-01$ \\
$3.076485168049643+00$ & $5.641660683767700-02$ \\
$5.140112867471345+00$ & $7.486688107514948-03$ \\
$7.833343693912404+00$ & $5.313425045101793-04$ \\
$1.126446663794674+01$ & $1.824683798220893-05$ \\
$1.561778565480894+01$ & $2.548106024523016-07$ \\
$2.125071587180707+01$ & $1.034504058033080-09$ \\
$2.908192754018544+01$ & $5.251417484918089-13$
\end{tabular}

Tabla 10.2: Cuadratura Gaussiana de diez puntos para la función de peso $w(x)=$ $K_{0}(x)$. 


\begin{tabular}{cc}
$\alpha_{n}$ & $\beta_{n}$ \\
\hline $0.127323954473516+01$ & $0.157079632679490+01$ \\
$0.334375770060194+01$ & $0.137886106172260+01$ \\
$0.537466242867702+01$ & $0.479184076622125+01$ \\
$0.739261165744769+01$ & $0.102226950377245+02$ \\
$0.940460447497128+01$ & $0.176643030917875+02$ \\
$0.114133180332566+02$ & $0.271130893358387+02$ \\
$0.134200104312221+02$ & $0.385670295898178+02$ \\
$0.154253567158969+02$ & $0.520248713474705+02$ \\
$0.174297544317213+02$ & $0.674857857597322+02$ \\
$0.194334544450458+02$ & $0.849491950074074+02$ \\
$0.214366237310800+02$ & $0.104414679261394+03$ \\
$0.234393782370461+02$ & $0.125881923092245+03$ \\
$0.254418012988880+02$ & $0.149350682880638+03$ \\
$0.274439545485356+02$ & $0.174820766084342+03$ \\
$0.294458846755186+02$ & $0.202292017536461+03$ \\
$0.314476277825718+02$ & $0.231764310091248+03$ \\
$0.334492122846222+02$ & $0.263237538056313+03$ \\
$0.354506608934532+02$ & $0.296711612467175+03$ \\
$0.374519920101924+02$ & $0.332186457613645+03$ \\
$0.394532207236237+02$ & $0.369662008437541+03$ \\
$0.414543595397074+02$ & $0.409138208550024+03$ \\
$0.434554189238418+02$ & $0.450615008698038+03$ \\
$0.454564077101366+02$ & $0.494092365561941+03$ \\
$0.474573334146042+02$ & $0.539570240801173+03$ \\
$0.494582024778364+02$ & $0.587048600288326+03$ \\
$0.514590204551974+02$ & $0.636527413488160+03$ \\
$0.534597921674419+02$ & $0.688006652949456+03$ \\
$0.554605218211384+02$ & $0.741486293885662+03$ \\
$0.574612131058024+02$ & $0.796966313826079+03$ \\
$0.594618692728830+02$ & $0.854446692323645+03$ \\
$0.614624932004795+02$ & $0.913927410708467+03$ \\
$0.634630874467395+02$ & $0.975408451878670+03$ \\
$0.654636542942050+02$ & $0.103888980012186+04$ \\
$0.674641957868683+02$ & $0.110437144096191+04$ \\
$0.694647137613125+02$ & $0.117185336102684+04$ \\
$0.714652098730208+02$ & $0.124133554793427+04$ \\
$0.734656856187183+02$ & $0.131281799019178+04$ \\
$0.754661423554324+02$ & $0.138630067710976+04$ \\
$0.774665813168291+02$ & $0.146178359872497+04$ \\
$0.794670036272716+02$ & $0.153926674573321+04$
\end{tabular}




\begin{tabular}{cc}
$\alpha_{n}$-continuación- \\
$\beta_{n}$ \\
\hline $0.814674103139695+02$ & $0.161875010942985+04$ \\
$0.834678023175156+02$ & $0.170023368165697+04$ \\
$0.854681805010591+02$ & $0.178371745475652+04$ \\
$0.874685456583183+02$ & $0.186920142152836+04$ \\
$0.894688985206021+02$ & $0.195668557519285+04$ \\
$0.914692397629829+02$ & $0.204616990935719+04$ \\
$0.934695700097388+02$ & $0.213765441798520+04$ \\
$0.954698898391654+02$ & $0.223113909537006+04$ \\
$0.974701997878424+02$ & $0.232662393610966+04$ \\
$0.994705003544249+02$ & $0.242410893508429+04$ \\
$0.101470792003023+03$ & $0.252359408743640+04$ \\
$0.103471075166220+03$ & $0.262507938855213+04$ \\
$0.105471350247773+03$ & $0.272856483404457+04$ \\
$0.107471617625037+03$ & $0.283405041973839+04$ \\
$0.109471877651149+03$ & $0.294153614165582+04$ \\
$0.111472130656982+03$ & $0.305102199600380+04$ \\
$0.113472376952928+03$ & $0.316250797916222+04$ \\
$0.115472616830493+03$ & $0.327599408767301+04$ \\
$0.117472850563752+03$ & $0.339148031823019+04$ \\
$0.119473078410666+03$ & $0.350896666767070+04$ \\
$0.121473300614284+03$ & $0.362845313296580+04$ \\
$0.123473517403827+03$ & $0.374993971121333+04$ \\
$0.125473728995689+03$ & $0.387342639963031+04$ \\
$0.127473935594343+03$ & $0.399891319554628+04$ \\
$0.129474137393173+03$ & $0.412640009639699+04$ \\
$0.131474334575236+03$ & $0.425588709971858+04$ \\
$0.133474527313960+03$ & $0.438737420314217+04$ \\
$0.135474715773784+03$ & $0.452086140438880+04$ \\
$0.137474900110753+03$ & $0.465634870126473+04$ \\
$0.139475080473055+03$ & $0.479383609165702+04$ \\
$0.141475257001528+03$ & $0.493332357352947+04$ \\
$0.143475429830119+03$ & $0.507481114491872+04$ \\
$0.145475599086312+03$ & $0.521829880393069+04$ \\
$0.147475764891525+03$ & $0.536378654873717+04$ \\
$0.149475927361475+03$ & $0.551127437757270+04$ \\
$0.151476086606518+03$ & $0.566076228873154+04$ \\
$0.153476242731963+03$ & $0.581225028056494+04$ \\
$0.155476395838366+03$ & $0.596573835147846+04$ \\
$0.157476546021805+03$ & $0.612122649992951+04$ \\
$0.159476693374127+03$ & $0.627871472442503+04$
\end{tabular}

Tabla 10.3: Coeficientes de recurrencia para la función de peso $w(x)=x K_{1}(x)$. 


\begin{tabular}{cc} 
Abscisas & Pesos \\
\hline $3.236388725848161-01$ & $7.312100574923358-01$ \\
$1.619014763308521+00$ & $6.794547596829900-01$ \\
$3.953726791864134+00$ & $1.519246960873696-01$ \\
$7.591825239709581+00$ & $8.148784443736022-03$ \\
$1.330067013896603+01$ & $5.802908846518882-05$
\end{tabular}

Tabla 10.4: Cuadratura Gaussiana de cinco puntos para la función de peso $w(x)=x K_{1}(x)$.

\begin{tabular}{cc} 
Abscisas & Pesos \\
\hline $1.630098883239737-01$ & $3.983898903221983-01$ \\
$8.279820439457481-01$ & $6.188755246031987-01$ \\
$1.991851553324522+00$ & $3.988025948990537-01$ \\
$3.670324216331792+00$ & $1.304122723114443-01$ \\
$5.904745221614970+00$ & $2.230190835044418-02$ \\
$8.763650402364749+00$ & $1.934604861616689-03$ \\
$1.235717198742878+01$ & $7.827069275976689-05$ \\
$1.687253706118580+01$ & $1.255000393068355-06$ \\
$2.267222714832694+01$ & $5.750518879552425-09$ \\
$3.068727034072851+01$ & $3.269064931048025-12$
\end{tabular}

Tabla 10.5: Cuadratura Gaussiana de diez puntos para la función de peso $w(x)=$ $x K_{1}(x)$. 


\begin{tabular}{cc}
$\alpha_{n}$ & $\beta_{n}$ \\
\hline $0.169765272631355+01$ & $0.471238898038469+01$ \\
$0.391316319224135+01$ & $0.211797522084017+01$ \\
$0.601902264806164+01$ & $0.629066916461253+01$ \\
$0.808257796620297+01$ & $0.125234083276395+02$ \\
$0.101258268619112+02$ & $0.207962567230252+02$ \\
$0.121577020559160+02$ & $0.310961791900020+02$ \\
$0.141824830207253+02$ & $0.434154392524348+02$ \\
$0.162024870635148+02$ & $0.577492726476044+02$ \\
$0.182190892357882+02$ & $0.740945744375976+02$ \\
$0.202331641814978+02$ & $0.924492086537446+02$ \\
$0.222452989905597+02$ & $0.112811636247757+03$ \\
$0.242559048475672+02$ & $0.135180705106504+03$ \\
$0.262652797762908+02$ & $0.159555525501625+03$ \\
$0.282736459490983+02$ & $0.185935392645514+03$ \\
$0.302811729335535+02$ & $0.214319736441523+03$ \\
$0.322879927547966+02$ & $0.244708087632553+03$ \\
$0.342942099837966+02$ & $0.277100054240450+03$ \\
$0.362999086884657+02$ & $0.311495304698667+03$ \\
$0.383051573421159+02$ & $0.347893555478696+03$ \\
$0.403100123645435+02$ & $0.386294561819155+03$ \\
$0.423145207253024+02$ & $0.426698110650425+03$ \\
$0.443187218898510+02$ & $0.469104015106999+03$ \\
$0.463226492963906+02$ & $0.513512110210136+03$ \\
$0.483263314917656+02$ & $0.559922249427933+03$ \\
$0.503297930158449+02$ & $0.608334301903245+03$ \\
$0.523330550977516+02$ & $0.658748150196855+03$ \\
$0.543361362095494+02$ & $0.71163688433074+03$ \\
$0.563390525106771+02$ & $0.765580820763115+03$ \\
$0.583418182077559+02$ & $0.821999460081951+03$ \\
$0.603444458481941+02$ & $0.880419526949235+03$ \\
$0.623469465615325+02$ & $0.940840948675886+03$ \\
$0.643493302591893+02$ & $0.100326365854623+04$ \\
$0.663516058008273+02$ & $0.106768759515183+04$ \\
$0.683537811337464+02$ & $0.113411270181804+04$ \\
$0.703558634103257+02$ & $0.120253892610789+04$ \\
$0.723578590874887+02$ & $0.127296621939093+04$ \\
$0.743597740113597+02$ & $0.134539453646700+04$ \\
$0.763616134896472+02$ & $0.141982383523647+04$ \\
$0.783633823538067+02$ & $0.149625407641020+04$ \\
$0.803650850126454+02$ & $0.157468522325356+04$
\end{tabular}




\begin{tabular}{cc}
-continuación- \\
$\alpha_{n}$ & $\beta_{n}$ \\
\hline $0.823667254987287+02$ & $0.165511724135956+04$ \\
$0.843683075087034+02$ & $0.173755009844741+04$ \\
$0.863698344384597+02$ & $0.182198376418286+04$ \\
$0.883713094138941+02$ & $0.190841821001765+04$ \\
$0.903727353179103+02$ & $0.199685340904563+04$ \\
$0.923741148141903+02$ & $0.208728933587336+04$ \\
$0.943754503681817+02$ & $0.217972596650367+04$ \\
$0.963767442656804+02$ & $0.227416327823042+04$ \\
$0.983779986293256+02$ & $0.237060124954322+04$ \\
$0.100379215433281+03$ & $0.246903986004111+04$ \\
$0.102380396516332+03$ & $0.256947909035402+04$ \\
$0.104381543593596+03$ & $0.267191892207121+04$ \\
$0.106382658267021+03$ & $0.277635933767608+04$ \\
$0.108383742034813+03$ & $0.288280032048649+04$ \\
$0.110384796299916+03$ & $0.299124185460010+04$ \\
$0.112385822377669+03$ & $0.310168392484431+04$ \\
$0.114386821502715+03$ & $0.321412651673014+04$ \\
$0.116387794835261+03$ & $0.332856961640993+04$ \\
$0.118388743466750+03$ & $0.344501321063820+04$ \\
$0.120389668425019+03$ & $0.356345728673569+04$ \\
$0.122390570678983+03$ & $0.368390183255595+04$ \\
$0.124391451142916+03$ & $0.380634683645457+04$ \\
$0.126392310680345+03$ & $0.393079228726055+04$ \\
$0.128393150107621+03$ & $0.405723817424980+04$ \\
$0.130393970197177+03$ & $0.418568448712048+04$ \\
$0.132394771680529+03$ & $0.431613121597010+04$ \\
$0.134395555251013+03$ & $0.444857835127417+04$ \\
$0.136396321566316+03$ & $0.458302588386626+04$ \\
$0.138397071250797+03$ & $0.471947380491950+04$ \\
$0.140397804897630+03$ & $0.485792210592916+04$ \\
$0.142398523070775+03$ & $0.499837077869644+04$ \\
$0.144399226306807+03$ & $0.514081981531330+04$ \\
$0.146399915116600+03$ & $0.528526920814821+04$ \\
$0.148400589986890+03$ & $0.543171894983278+04$ \\
$0.150401251381721+03$ & $0.558016903324927+04$ \\
$0.152401899743790+03$ & $0.573061945151875+04$ \\
$0.154402535495690+03$ & $0.588307019799009+04$ \\
$0.156403159041073+03$ & $0.603752126622951+04$ \\
$0.158403770765723+03$ & $0.619397265001076+04$ \\
$0.160404371038567+03$ & $0.635242434330585+04$
\end{tabular}

Tabla 10.6: Coeficientes de recurrencia para la función de peso $w(x)=x^{2} K_{2}(x)$. 


\begin{tabular}{cc} 
Abscisas & Pesos \\
\hline $4.028804443733526-01$ & $1.920550470548815+00$ \\
$1.943507878887528+00$ & $2.160291525691454+00$ \\
$4.554322827789002+00$ & $5.933454933664700-01$ \\
$8.469268246102408+00$ & $3.788834268079449-02$ \\
$1.446826399757837+01$ & $3.131480971565248-04$
\end{tabular}

Tabla 10.7: Cuadratura Gaussiana de cinco puntos para la función de peso $w(x)=x^{2} K_{2}(x)$.

\begin{tabular}{cc} 
Abscisas & Pesos \\
\hline $1.929214440099163-01$ & $9.674203346551806-01$ \\
$9.711682620400458-01$ & $1.730934120551488+00$ \\
$2.285663116948677+00$ & $1.357381826605418+00$ \\
$4.122501924760546+00$ & $5.368351816130900-01$ \\
$6.514375363009271+00$ & $1.084564311211495-01$ \\
$9.528225287750705+00$ & $1.085529142372329-02$ \\
$1.327505302523484+01$ & $4.968716782625190-04$ \\
$1.794445582806802+01$ & $8.877849556293435-06$ \\
$2.390356515461224+01$ & $4.485880813299287-08$ \\
$3.209523954573858+01$ & $2.801400939011396-11$
\end{tabular}

Tabla 10.8: Cuadratura Gaussiana de diez puntos para la función de peso $w(x)=$ $x^{2} K_{2}(x)$. 


\begin{tabular}{cc}
$\alpha_{n}$ & $\beta_{n}$ \\
\hline $0.203718327157626+01$ & $0.235619449019234+02$ \\
$0.439628819543049+01$ & $0.284988431800984+01$ \\
$0.658112833097809+01$ & $0.773524588487516+01$ \\
$0.869415512267929+01$ & $0.147275376046612+02$ \\
$0.107722988878063+02$ & $0.237930333417394+02$ \\
$0.128307090169876+02$ & $0.349073989021315+02$ \\
$0.148766536092345+02$ & $0.480565934338115+02$ \\
$0.169140932710264+02$ & $0.632322122408102+02$ \\
$0.189454028087682+02$ & $0.804288425094676+02$ \\
$0.209721108074902+02$ & $0.996427544012018+02$ \\
$0.229952551740501+02$ & $0.120871236165510+03$ \\
$0.250155704113317+02$ & $0.144112234163521+03$ \\
$0.270335937196047+02$ & $0.169364143983965+03$ \\
$0.290497287119940+02$ & $0.196625681351078+03$ \\
$0.310642855188989+02$ & $0.225895798003111+03$ \\
$0.330775070249421+02$ & $0.257173624460508+03$ \\
$0.350895865967512+02$ & $0.290458429707600+03$ \\
$0.371006803943645+02$ & $0.325749591970449+03$ \\
$0.391109161256764+02$ & $0.363046577031706+03$ \\
$0.411203994013383+02$ & $0.402348921814664+03$ \\
$0.431292184324747+02$ & $0.443656221741382+03$ \\
$0.451374475599770+02$ & $0.486968120850483+03$ \\
$0.471451499446751+02$ & $0.532284303969402+03$ \\
$0.491523796448474+02$ & $0.579604490440382+03$ \\
$0.511591832397038+02$ & $0.628928429038146+03$ \\
$0.531656011118203+02$ & $0.680255893813124+03$ \\
$0.551716684702153+02$ & $0.733586680661790+03$ \\
$0.571774161739498+02$ & $0.788920604474167+03$ \\
$0.591828714007084+02$ & $0.846257496743838+03$ \\
$0.611880581937493+02$ & $0.905597203551846+03$ \\
$0.631929979125706+02$ & $0.966939583855286+03$ \\
$0.651977096067272+02$ & $0.103028450802604+04$ \\
$0.672022103278381+02$ & $0.109563185659631+04$ \\
$0.692065153915244+02$ & $0.116298151917628+04$ \\
$0.712106385985131+02$ & $0.123233339351574+04$ \\
$0.732145924222317+02$ & $0.130368738468702+04$ \\
$0.752183881687396+02$ & $0.137704340437060+04$ \\
$0.772220361136965+02$ & $0.145240137022786+04$ \\
$0.792255456201680+02$ & $0.152976120534856+04$ \\
$0.812289252403625+02$ & $0.160912283776224+04$
\end{tabular}




\begin{tabular}{cc}
$\alpha_{n}$ & -continuación- \\
\hline $0.832321828038274+02$ & $0.169048620000481+04$ \\
$0.852353254941869+02$ & $0.177385122873286+04$ \\
$0.872383599161422+02$ & $0.185921786437949+04$ \\
$0.892412921541606+02$ & $0.194658605084617+04$ \\
$0.912441278240482+02$ & $0.203595573522629+04$ \\
$0.932468721184032+02$ & $0.212732686755641+04$ \\
$0.952495298467906+02$ & $0.222069940059184+04$ \\
$0.972521054713495+02$ & $0.231607328960389+04$ \\
$0.992546031384325+02$ & $0.241344849219603+04$ \\
$0.101257026706792+03$ & $0.251282496813711+04$ \\
$0.103259379772748+03$ & $0.261420267920948+04$ \\
$0.105261665692717+03$ & $0.271758158907065+04$ \\
$0.107263887603412+03$ & $0.282296166312691+04$ \\
$0.109266048440014+03$ & $0.293034286841765+04$ \\
$0.111268150952521+03$ & $0.303972517350943+04$ \\
$0.113270197720523+03$ & $0.315110854839864+04$ \\
$0.115272191166542+03$ & $0.326449296442207+04$ \\
$0.117274133568131+03$ & $0.337987839417450+04$ \\
$0.119276027068847+03$ & $0.349726481143268+04$ \\
$0.121277873688221+03$ & $0.361665219108520+04$ \\
$0.123279675330844+03$ & $0.373804050906755+04$ \\
$0.125281433794646+03$ & $0.386142974230196+04$ \\
$0.127283150778458+03$ & $0.398681986864172+04$ \\
$0.129284827888932+03$ & $0.411421086681936+04$ \\
$0.131286466646875+03$ & $0.424360271639855+04$ \\
$0.133288068493061+03$ & $0.437499539772929+04$ \\
$0.135289634793570+03$ & $0.450838889190623+04$ \\
$0.137291166844694+03$ & $0.464378318072969+04$ \\
$0.139292665877460+03$ & $0.478117824666935+04$ \\
$0.141294133061792+03$ & $0.492057407283026+04$ \\
$0.143295569510364+03$ & $0.506197064292106+04$ \\
$0.145296976282150+03$ & $0.520536794122420+04$ \\
$0.147298354385711+03$ & $0.535076595256805+04$ \\
$0.149299704782245+03$ & $0.549816466230070+04$ \\
$0.151301028388406+03$ & $0.564756405626533+04$ \\
$0.153302326078927+03$ & $0.579896412077719+04$ \\
$0.155303598689053+03$ & $0.595236484260173+04$ \\
$0.157304847016803+03$ & $0.610776620893423+04$ \\
$0.159306071825082+03$ & $0.626516820738045+04$ \\
$0.161307273843638+03$ & $0.642457082593844+04$
\end{tabular}

Tabla 10.9: Coeficientes de recurrencia para la función de peso $w(x)=x^{3} K_{3}(x)$. 


\begin{tabular}{cc} 
Abscisas & Pesos \\
\hline $4.606906809186083-01$ & $8.909700140124831+00$ \\
$2.206118326958725+00$ & $1.097673030814545+01$ \\
$5.066858223535144+00$ & $3.425778321623442+00$ \\
$9.238246176753681+00$ & $2.474459730151987-01$ \\
$1.550914040030430+01$ & $2.290159014531770-03$
\end{tabular}

Tabla 10.10: Cuadratura Gaussiana de cinco puntos para la función de peso $w(x)=x^{3} K_{3}(x)$.

\begin{tabular}{cc} 
Abscisas & Pesos \\
\hline $2.140458170312080-01$ & $4.316589251175842+00$ \\
$1.079818573263136+00$ & $8.149254495909784+00$ \\
$2.525180308440953+00$ & $7.104060586848880+00$ \\
$4.507294968400214+00$ & $3.181611792741240+00$ \\
$7.046779907534519+00$ & $7.252284961629501-01$ \\
$1.020741334175151+01$ & $8.102636014962487-02$ \\
$1.410040013700009+01$ & $4.093520106828389-03$ \\
$1.891735417959003+01$ & $7.995980810871634-05$ \\
$2.502977930588859+01$ & $4.387231804682895-07$ \\
$3.339195678307718+01$ & $2.970102160416987-10$
\end{tabular}

Tabla 10.11: Cuadratura Gaussiana de diez puntos para la función de peso $w(x)=x^{3} K_{3}(x)$. 


\section{Apéndice $B$}

Se presentan las gráficas correspondientes a los polinomios ortogonales con respecto a funciones de peso $w(x)=K_{0}(x), w(x)=x K_{1}(x), w(x)=x^{2} K_{2}(x)$ y $w(x)=x^{3} K_{3}(x)$.

11.1 Gráficas de polinomios ortogonales para $w(x)=$ $x^{m} K_{m}(x)$.
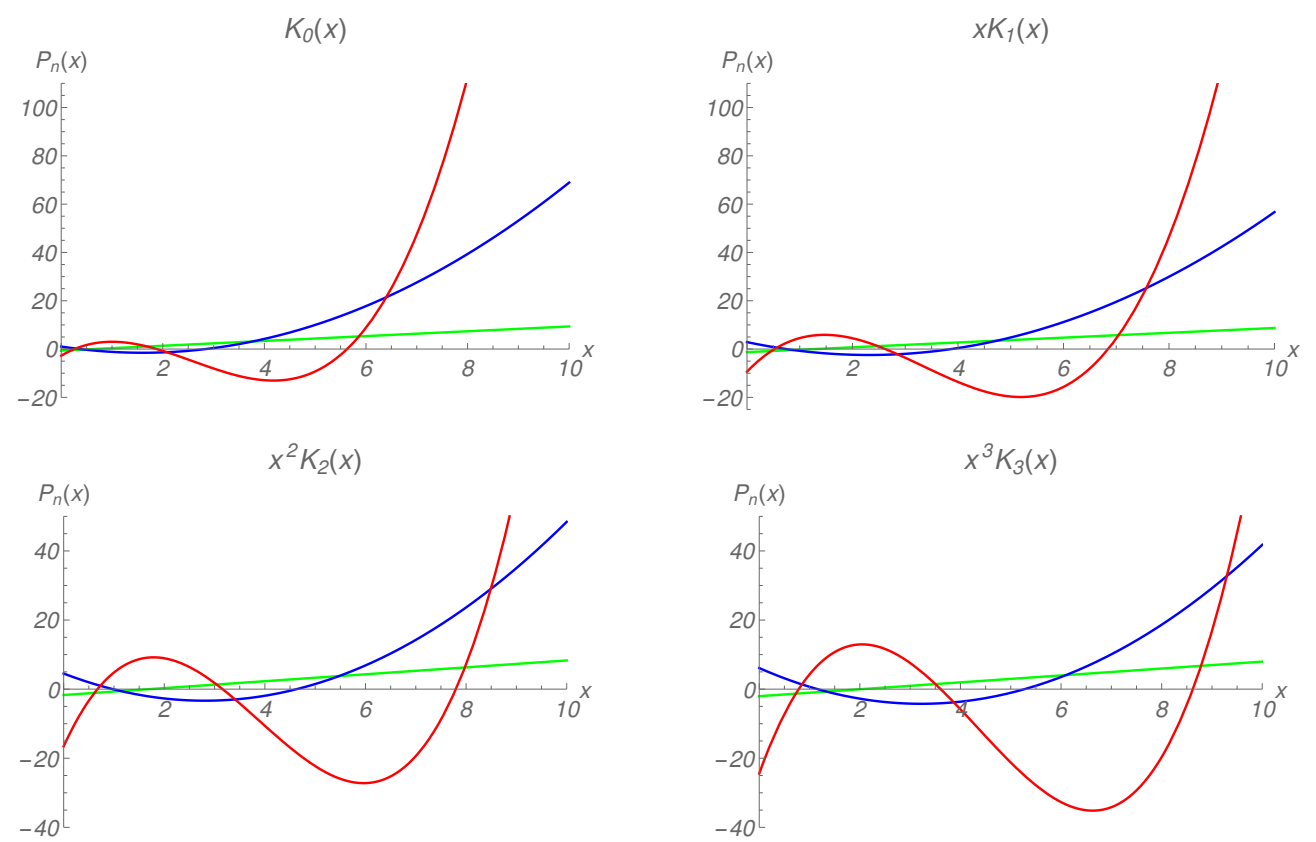

Figura 11.1: Gráficas de los tres primeros polinomios ortogonales en el intervalo $0 \leq x \leq 10$. El polinomio de primer grado $P_{1}(x)$ (verde), el polinomio de segundo grado $P_{2}(x)$ (azul) y el polinomio de tercer grado $P_{3}(x)$ (rojo). 


\section{Apéndice $\mathrm{C}$}

12.1 Coeficientes de recurrencia y cuadraturas Gaussianas para funciones de peso $w(x)=e^{-x} \Theta_{l}(x)$ para $l=0, l=1, l=2$ y $l=3$ con el polinomio de Bessel inverso $\Theta_{l}(x)$.

\begin{tabular}{cc}
$\alpha_{n}$ & $\beta_{n}$ \\
\hline $0.100000000000000+01$ & $0.100000000000000+01$ \\
$0.300000000000000+01$ & $0.100000000000000+01$ \\
$0.500000000000000+01$ & $0.400000000000000+01$ \\
$0.700000000000000+01$ & $0.900000000000000+01$ \\
$0.900000000000000+01$ & $0.160000000000000+02$ \\
$0.110000000000000+02$ & $0.250000000000000+02$ \\
$0.130000000000000+02$ & $0.360000000000000+02$ \\
$0.150000000000000+02$ & $0.490000000000000+02$ \\
$0.170000000000000+02$ & $0.640000000000000+02$ \\
$0.190000000000000+02$ & $0.810000000000000+02$ \\
$0.210000000000000+02$ & $0.100000000000000+03$ \\
$0.230000000000000+02$ & $0.121000000000000+03$ \\
$0.250000000000000+02$ & $0.144000000000000+03$ \\
$0.270000000000000+02$ & $0.169000000000000+03$ \\
$0.290000000000000+02$ & $0.19600000000000+03$ \\
$0.310000000000000+02$ & $0.225000000000000+03$ \\
$0.330000000000000+02$ & $0.25600000000000+03$ \\
$0.350000000000000+02$ & $0.289000000000000+03$ \\
$0.370000000000000+02$ & $0.324000000000000+03$ \\
$0.390000000000000+02$ & $0.361000000000000+03$ \\
$0.410000000000000+02$ & $0.400000000000000+03$ \\
$0.430000000000000+02$ & $0.441000000000000+03$ \\
$0.450000000000000+02$ & $0.484000000000000+03$ \\
$0.470000000000000+02$ & $0.529000000000000+03$ \\
$0.490000000000000+02$ & $0.576000000000000+03$ \\
$0.510000000000000+02$ & $0.625000000000000+03$ \\
$0.530000000000000+02$ & $0.676000000000000+03$ \\
$0.550000000000000+02$ & $0.72900000000000+03$ \\
$0.570000000000000+02$ & $0.784000000000000+03$ \\
$0.590000000000000+02$ & $0.841000000000000+03$ \\
$0.610000000000000+02$ & $0.900000000000000+03$ \\
$0.630000000000000+02$ & $0.961000000000000+03$ \\
$0.650000000000000+02$ & $0.102400000000000+04$ \\
$0.670000000000000+02$ & $0.108900000000000+04$
\end{tabular}


-continuación-

\begin{tabular}{cc}
$\alpha_{n}$ & $\beta_{n}$ \\
\hline $0.690000000000000+02$ & $0.115600000000000+04$ \\
$0.710000000000000+02$ & $0.122500000000000+04$ \\
$0.730000000000000+02$ & $0.129600000000000+04$ \\
$0.750000000000000+02$ & $0.136900000000000+04$ \\
$0.770000000000000+02$ & $0.144400000000000+04$ \\
$0.790000000000000+02$ & $0.152100000000000+04$ \\
$0.810000000000000+02$ & $0.160000000000000+04$ \\
$0.830000000000000+02$ & $0.168100000000000+04$ \\
$0.850000000000000+02$ & $0.176400000000000+04$ \\
$0.870000000000000+02$ & $0.184900000000000+04$ \\
$0.890000000000000+02$ & $0.193600000000000+04$ \\
$0.910000000000000+02$ & $0.202500000000000+04$ \\
$0.930000000000000+02$ & $0.211600000000000+04$ \\
$0.950000000000000+02$ & $0.220900000000000+04$ \\
$0.970000000000000+02$ & $0.230400000000000+04$ \\
$0.990000000000000+02$ & $0.240100000000000+04$ \\
$0.101000000000000+03$ & $0.250000000000000+04$ \\
$0.103000000000000+03$ & $0.260100000000000+04$ \\
$0.105000000000000+03$ & $0.270400000000000+04$ \\
$0.107000000000000+03$ & $0.280900000000000+04$ \\
$0.109000000000000+03$ & $0.291600000000000+04$ \\
$0.111000000000000+03$ & $0.302500000000000+04$ \\
$0.113000000000000+03$ & $0.313600000000000+04$ \\
$0.115000000000000+03$ & $0.324900000000000+04$ \\
$0.117000000000000+03$ & $0.336400000000000+04$ \\
$0.119000000000000+03$ & $0.348100000000000+04$ \\
$0.121000000000000+03$ & $0.360000000000000+04$ \\
$0.123000000000000+03$ & $0.372100000000000+04$ \\
$0.125000000000000+03$ & $0.384400000000000+04$ \\
$0.127000000000000+03$ & $0.396900000000000+04$ \\
$0.129000000000000+03$ & $0.409600000000000+04$ \\
$0.131000000000000+03$ & $0.422500000000000+04$ \\
$0.133000000000000+03$ & $0.435600000000000+04$ \\
$0.135000000000000+03$ & $0.448900000000000+04$ \\
$0.137000000000000+03$ & $0.462400000000000+04$ \\
$0.139000000000000+03$ & $0.476100000000000+04$ \\
$0.141000000000000+03$ & $0.490000000000000+04$ \\
$0.143000000000000+03$ & $0.504100000000000+04$ \\
$0.145000000000000+03$ & $0.518400000000000+04$ \\
$0.147000000000000+03$ & $0.532900000000000+04$ \\
$0.149000000000000+03$ & $0.547600000000000+04$ \\
$0.151000000000000+03$ & $0.562500000000000+04$ \\
$0.153000000000000+03$ & $0.577600000000000+04$
\end{tabular}


-continación-

\begin{tabular}{cc}
$\alpha_{n}$ & $\beta_{n}$ \\
\hline $0.155000000000000+03$ & $0.592900000000000+04$ \\
$0.157000000000000+03$ & $0.608400000000000+04$ \\
$0.159000000000000+03$ & $0.624100000000000+04$ \\
$0.161000000000000+03$ & $0.640000000000000+04$ \\
$0.163000000000000+03$ & $0.656100000000000+04$ \\
$0.165000000000000+03$ & $0.672400000000000+04$ \\
$0.167000000000000+03$ & $0.688900000000000+04$ \\
$0.169000000000000+03$ & $0.705600000000000+04$ \\
$0.171000000000000+03$ & $0.722500000000000+04$ \\
$0.173000000000000+03$ & $0.739600000000000+04$ \\
$0.175000000000000+03$ & $0.756900000000000+04$ \\
$0.177000000000000+03$ & $0.774400000000000+04$ \\
$0.179000000000000+03$ & $0.792100000000000+04$ \\
$0.181000000000000+03$ & $0.810000000000000+04$ \\
$0.183000000000000+03$ & $0.828100000000000+04$ \\
$0.185000000000000+03$ & $0.846400000000000+04$ \\
$0.187000000000000+03$ & $0.864900000000000+04$ \\
$0.189000000000000+03$ & $0.883600000000000+04$ \\
$0.191000000000000+03$ & $0.902500000000000+04$ \\
$0.193000000000000+03$ & $0.921600000000000+04$ \\
$0.195000000000000+03$ & $0.940900000000000+04$ \\
$0.197000000000000+03$ & $0.960400000000000+04$ \\
$0.199000000000000+03$ & $0.980100000000000+04$ \\
$0.201000000000000+03$ & $0.100000000000000+05$ \\
$0.203000000000000+03$ & $0.102010000000000+05$ \\
$0.205000000000000+03$ & $0.104040000000000+05$ \\
$0.207000000000000+03$ & $0.106090000000000+05$ \\
$0.209000000000000+03$ & $0.108160000000000+05$ \\
$0.211000000000000+03$ & $0.110250000000000+05$ \\
$0.213000000000000+03$ & $0.112360000000000+05$ \\
$0.215000000000000+03$ & $0.114490000000000+05$ \\
$0.217000000000000+03$ & $0.116640000000000+05$ \\
$0.219000000000000+03$ & $0.118810000000000+05$ \\
$0.221000000000000+03$ & $0.121000000000000+05$ \\
$0.223000000000000+03$ & $0.123210000000000+05$ \\
$0.225000000000000+03$ & $0.125440000000000+05$ \\
$0.227000000000000+03$ & $0.127690000000000+05$ \\
$0.229000000000000+03$ & $0.129960000000000+05$ \\
$0.231000000000000+03$ & $0.132250000000000+05$ \\
$0.233000000000000+03$ & $0.134560000000000+05$ \\
$0.235000000000000+03$ & $0.136890000000000+05$ \\
$0.237000000000000+03$ & $0.139240000000000+05$ \\
$0.239000000000000+03$ & $0.141610000000000+05$
\end{tabular}


-continuación-

\begin{tabular}{cc}
$\alpha_{n}$ & $\beta_{n}$ \\
\hline $0.241000000000000+03$ & $0.144000000000000+05$ \\
$0.243000000000000+03$ & $0.146410000000000+05$ \\
$0.245000000000000+03$ & $0.148840000000000+05$ \\
$0.247000000000000+03$ & $0.151290000000000+05$ \\
$0.249000000000000+03$ & $0.153760000000000+05$ \\
$0.251000000000000+03$ & $0.156250000000000+05$ \\
$0.253000000000000+03$ & $0.158760000000000+05$ \\
$0.255000000000000+03$ & $0.161290000000000+05$ \\
$0.257000000000000+03$ & $0.163840000000000+05$ \\
$0.259000000000000+03$ & $0.166410000000000+05$ \\
$0.261000000000000+03$ & $0.169000000000000+05$ \\
$0.263000000000000+03$ & $0.171610000000000+05$ \\
$0.265000000000000+03$ & $0.174240000000000+05$ \\
$0.267000000000000+03$ & $0.176890000000000+05$ \\
$0.269000000000000+03$ & $0.179560000000000+05$ \\
$0.271000000000000+03$ & $0.182250000000000+05$ \\
$0.273000000000000+03$ & $0.184960000000000+05$ \\
$0.275000000000000+03$ & $0.187690000000000+05$ \\
$0.277000000000000+03$ & $0.190440000000000+05$ \\
$0.279000000000000+03$ & $0.193210000000000+05$ \\
$0.281000000000000+03$ & $0.196000000000000+05$ \\
$0.283000000000000+03$ & $0.198810000000000+05$ \\
$0.285000000000000+03$ & $0.201640000000000+05$ \\
$0.287000000000000+03$ & $0.204490000000000+05$ \\
$0.289000000000000+03$ & $0.207360000000000+05$ \\
$0.291000000000000+03$ & $0.210250000000000+05$ \\
$0.293000000000000+03$ & $0.213160000000000+05$ \\
$0.295000000000000+03$ & $0.216090000000000+05$ \\
$0.297000000000000+03$ & $0.219040000000000+05$ \\
$0.299000000000000+03$ & $0.222010000000000+05$
\end{tabular}

Tabla 12.1: Coeficientes de recurrencia para la función de peso de Laguerre $w(x)=e^{-x}$. 


\begin{tabular}{cc} 
Abscisas & Pesos \\
\hline $2.635603197181409-01$ & $5.217556105828087-01$ \\
$1.413403059106517+00$ & $3.986668110831759-01$ \\
$3.596425771040722+00$ & $7.594244968170760-01$ \\
$7.085810005858838+00$ & $3.611758679922048-03$ \\
$1.264080084427578+01$ & $2.336997238577623-05$
\end{tabular}

Tabla 12.2: Cuadratura Gaussiana de cinco puntos para la función de peso de Laguerre $w(x)=e^{-x}$.

\begin{tabular}{cc} 
Abscisas & Pesos \\
\hline $1.377934705404924-01$ & $3.084411157650201-01$ \\
$7.294545495031705-01$ & $4.011199291552736-01$ \\
$1.808342901740316+00$ & $2.180682876118094-01$ \\
$3.401433697854900+00$ & $6.208745609867775-02$ \\
$5.552496140063804+00$ & $9.501516975181101-03$ \\
$8.330152746764497+00$ & $7.530083885875388-04$ \\
$1.184378583790007+01$ & $2.825923349599566-05$ \\
$1.627925783137810+01$ & $4.249313984962686-07$ \\
$2.199658581198076+01$ & $1.839564823979631-09$ \\
$2.992069701227389+01$ & $9.911827219609009-13$
\end{tabular}

Tabla 12.3: Cuadratura Gaussiana de diez puntos para la función de peso de Laguerre $w(x)=e^{-x}$. 


\begin{tabular}{cc}
$\alpha_{n}$ & $\beta_{n}$ \\
\hline $0.150000000000000+01$ & $0.200000000000000+01$ \\
$0.364285714285714+01$ & $0.175000000000000+01$ \\
$0.571008403361345+01$ & $0.555102040816327+01$ \\
$0.774992963692654+01$ & $0.113901384083045+02$ \\
$0.977681870794828+01$ & $0.192537716627367+02$ \\
$0.117964941659060+02$ & $0.291339729954529+02$ \\
$0.138116945914183+02$ & $0.410260614318552+02$ \\
$0.158238991080652+02$ & $0.549271227358904+02$ \\
$0.178339824233824+02$ & $0.708352365682654+02$ \\
$0.198424984942197+02$ & $0.887490727485758+02$ \\
$0.218498173407223+02$ & $0.108667670262942+03$ \\
$0.238561968298025+02$ & $0.130590310018693+03$ \\
$0.258618229692186+02$ & $0.154516438004406+03$ \\
$0.278668338096499+02$ & $0.180445616853798+03$ \\
$0.298713342887475+02$ & $0.208377494129913+03$ \\
$0.318754058129638+02$ & $0.238311780849870+03$ \\
$0.338791126496665+02$ & $0.270248236508800+03$ \\
$0.358825063140252+02$ & $0.304186658361952+03$ \\
$0.378856286548675+02$ & $0.340126873577114+03$ \\
$0.398885140727882+02$ & $0.378068733371600+03$ \\
$0.418911911452675+02$ & $0.418012108552907+03$ \\
$0.438936838377348+02$ & $0.459956886072569+03$ \\
$0.458960124199085+02$ & $0.503902966324856+03$ \\
$0.478981941687004+02$ & $0.549850261002121+03$ \\
$0.499002439141256+02$ & $0.597798691372375+03$ \\
$0.519021744680947+02$ & $0.647748186881475+03$ \\
$0.539039969646997+02$ & $0.699698684007959+03$ \\
$0.559057211328287+02$ & $0.753650125316711+03$ \\
$0.579073555164738+02$ & $0.809602458670727+03$ \\
$0.599089076542050+02$ & $0.867555636569763+03$ \\
$0.619103842264715+02$ & $0.927509615591718+03$ \\
$0.639117911773361+02$ & $0.989464355917840+03$ \\
$0.659131338157319+02$ & $0.105341982092686+04$ \\
$0.679144169001939+02$ & $0.111937597684614+04$ \\
$0.699156447101626+02$ & $0.118733279245044+04$ \\
$0.719168211063074+02$ & $0.125729023880038+04$ \\
$0.739179495818122+02$ & $0.132924828901459+04$ \\
$0.759190333061855+02$ & $0.140320691807025+04$ \\
$0.779200751628463+02$ & $0.147916610262784+04$ \\
$0.799210777815087+02$ & $0.155712582087662+04$
\end{tabular}


-continuación-

\begin{tabular}{cc}
$\alpha_{n}$ & $\beta_{n}$ \\
\hline $0.819220435661926+02$ & $0.163708605239787+04$ \\
$0.839229747195442+02$ & $0.171904677804353+04$ \\
$0.859238732640261+02$ & $0.180300797982808+04$ \\
$0.879247410604437+02$ & $0.188896964083199+04$ \\
$0.899255798241948+02$ & $0.197693174511527+04$ \\
$0.919263911395646+02$ & $0.206689427763981+04$ \\
$0.939271764723404+02$ & $0.215885722419948+04$ \\
$0.959279371809726+02$ & $0.225282057135704+04$ \\
$0.979286745264770+02$ & $0.234878430638708+04$ \\
$0.999293896812427+02$ & $0.244674841722432+04$ \\
$0.101930083736886+03$ & $0.254671289241657+04$ \\
$0.103930757711268+03$ & $0.264867772108201+04$ \\
$0.105931412554784+03$ & $0.275264289287014+04$ \\
$0.107932049156008+03$ & $0.285860839792617+04$ \\
$0.109932668346769+03$ & $0.296657422685837+04$ \\
$0.111933270906727+03$ & $0.307654037070817+04$ \\
$0.113933857567510+03$ & $0.318850682092270+04$ \\
$0.115934429016450+03$ & $0.330247356932947+04$ \\
$0.117934985899976+03$ & $0.341844060811315+04$ \\
$0.119935528826692+03$ & $0.353640792979403+04$ \\
$0.121936058370175+03$ & $0.365637552720823+04$ \\
$0.123936575071526+03$ & $0.377834339348929+04$ \\
$0.125937079441698+03$ & $0.390231152205117+04$ \\
$0.127937571963622+03$ & $0.402827990657250+04$ \\
$0.129938053094152+03$ & $0.415624854098186+04$ \\
$0.131938523265848+03$ & $0.428621741944422+04$ \\
$0.133938982888616+03$ & $0.441818653634822+04$ \\
$0.135939432351205+03$ & $0.455215588629433+04$ \\
$0.137939872022598+03$ & $0.468812546408383+04$ \\
$0.139940302253280+03$ & $0.482609526470853+04$ \\
$0.141940723376416+03$ & $0.496606528334109+04$ \\
$0.143941135708938+03$ & $0.510803551532603+04$ \\
$0.145941539552545+03$ & $0.525200595617129+04$ \\
$0.147941935194633+03$ & $0.539797660154029+04$ \\
$0.149942322909158+03$ & $0.554594744724449+04$ \\
$0.151942702957428+03$ & $0.569591848923644+04$ \\
$0.153943075588849+03$ & $0.584788972360319+04$ \\
$0.155943441041611+03$ & $0.600186114656012+04$ \\
$0.157943799543329+03$ & $0.615783275444512+04$ \\
$0.159944151311639+03$ & $0.631580454371312+04$
\end{tabular}


-continuación-

\begin{tabular}{cc}
$\alpha_{n}$ & $\beta_{n}$ \\
\hline $0.161944496554753+03$ & $0.647577651093088+04$ \\
$0.163944835471979+03$ & $0.663774865277216+04$ \\
$0.165945168254202+03$ & $0.680172096601311+04$ \\
$0.167945495084341+03$ & $0.696769344752784+04$ \\
$0.169945816137764+03$ & $0.713566609428439+04$ \\
$0.171946131582692+03$ & $0.730563890334076+04$ \\
$0.173946441580565+03$ & $0.747761187184126+04$ \\
$0.175946746286390+03$ & $0.765158499701298+04$ \\
$0.177947045849065+03$ & $0.782755827616247+04$ \\
$0.179947340411692+03$ & $0.800553170667260+04$ \\
$0.181947630111855+03$ & $0.818550528599956+04$ \\
$0.183947915081896+03$ & $0.836747901167001+04$ \\
$0.185948195449170+03$ & $0.855145288127839+04$ \\
$0.187948471336279+03$ & $0.873742689248433+04$ \\
$0.189948742861302+03$ & $0.892540104301021+04$ \\
$0.191949010138006+03$ & $0.911537533063884+04$ \\
$0.193949273276047+03$ & $0.930734975321122+04$ \\
$0.195949532381157+03$ & $0.950132430862446+04$ \\
$0.197949787555325+03$ & $0.969729899482969+04$ \\
$0.199950038896964+03$ & $0.989527380983021+04$ \\
$0.201950286501073+03$ & $0.100952487516796+05$ \\
$0.203950530459385+03$ & $0.102972238184800+05$ \\
$0.205950770860512+03$ & $0.105011990083804+05$ \\
$0.207951007790079+03$ & $0.107071743195751+05$ \\
$0.209951241330853+03$ & $0.109151497503021+05$ \\
$0.211951471562863+03$ & $0.111251252988415+05$ \\
$0.213951698563519+03$ & $0.113371009635145+05$ \\
$0.215951922407716+03$ & $0.115510767426816+05$ \\
$0.217952143167943+03$ & $0.117670526347418+05$ \\
$0.219952360914378+03$ & $0.119850286381307+05$ \\
$0.221952575714983+03$ & $0.122050047513200+05$ \\
$0.223952787635596+03$ & $0.124269809728159+05$ \\
$0.225952996740010+03$ & $0.126509573011582+05$ \\
$0.227953203090058+03$ & $0.128769337349192+05$ \\
$0.229953406745689+03$ & $0.131049102727029+05$ \\
$0.231953607765040+03$ & $0.133348869131436+05$ \\
$0.233953806204508+03$ & $0.135668636549053+05$ \\
$0.235954002118814+03$ & $0.138008404966808+05$ \\
$0.237954195561068+03$ & $0.140368174371907+05$ \\
$0.239954386582830+03$ & $0.142747944751827+05$
\end{tabular}


-continuación-

\begin{tabular}{cc}
$\alpha_{n}$ & $\beta_{n}$ \\
\hline $0.241954575234164+03$ & $0.145147716094308+05$ \\
$0.243954761563699+03$ & $0.147567488387343+05$ \\
$0.245954945618677+03$ & $0.150007261619175+05$ \\
$0.247955127445006+03$ & $0.152467035778285+05$ \\
$0.249955307087307+03$ & $0.154946810853390+05$ \\
$0.251955484588958+03$ & $0.157446586833431+05$ \\
$0.253955659992144+03$ & $0.159966363707574+05$ \\
$0.255955833337891+03$ & $0.162506141465196+05$ \\
$0.257956004666114+03$ & $0.165065920095883+05$ \\
$0.259956174015651+03$ & $0.167645699589426+05$ \\
$0.261956341424301+03$ & $0.170245479935812+05$ \\
$0.263956506928859+03$ & $0.172865261125221+05$ \\
$0.265956670565153+03$ & $0.175505043148018+05$ \\
$0.267956832368071+03$ & $0.178164825994753+05$ \\
$0.269956992371598+03$ & $0.180844609656152+05$ \\
$0.271957150608844+03$ & $0.183544394123113+05$ \\
$0.273957307112069+03$ & $0.186264179386703+05$ \\
$0.275957461912717+03$ & $0.189003965438153+05$ \\
$0.277957615041438+03$ & $0.191763752268853+05$ \\
$0.279957766528115+03$ & $0.194543539870350+05$ \\
$0.281957916401891+03$ & $0.197343328234341+05$ \\
$0.283958064691185+03$ & $0.200163117352672+05$ \\
$0.285958211423725+03$ & $0.203002907217335+05$ \\
$0.287958356626561+03$ & $0.205862697820459+05$ \\
$0.289958500326091+03$ & $0.208742489154313+05$ \\
$0.291958642548078+03$ & $0.211642281211299+05$ \\
$0.293958783317673+03$ & $0.214562073983951+05$ \\
$0.295958922659431+03$ & $0.217501867464929+05$ \\
$0.297959060597328+03$ & $0.220461661647018+05$ \\
$0.299959197154783+03$ & $0.223441456523126+05$
\end{tabular}

Tabla 12.4: Coeficientes de recurrencia para la función de peso $w(x)=e^{-x}(x+$ $1)$. 


\begin{tabular}{cc} 
Abscisas & Pesos \\
\hline $3.674290529550515-01$ & $8.617669902056584-01$ \\
$1.791726093042943+00$ & $8.989578828888994-01$ \\
$4.268337995162533+00$ & $2.258431597814047-01$ \\
$8.047833898869262+00$ & $1.332912541468874-02$ \\
$1.390436248131562+01$ & $1.028417093486880-04$
\end{tabular}

Tabla 12.5: Cuadratura Gaussiana de cinco puntos para la función de peso $w(x)=e^{-x}(x+1)$.

\begin{tabular}{cc} 
Abscisas & Pesos \\
\hline $1.797881951792418-01$ & $4.477173138673236-01$ \\
$9.057639228288455-01$ & $7.610542479174122-01$ \\
$2.147796401990209+00$ & $5.500932797342007-01$ \\
$3.907290042545831+00$ & $2.000200564822172-01$ \\
$6.221819170055896+00$ & $3.745314600380328-02$ \\
$9.159336958443133+00$ & $3.507848380915104-03$ \\
$1.283050114446644+01$ & $1.515231677324516-04$ \\
$1.742377422124112+01$ & $2.572034847206277-06$ \\
$2.330400253857352+01$ & $1.240414323015975-08$ \\
$3.140818570901279+01$ & $7.405040097785192-12$
\end{tabular}

Tabla 12.6: Cuadratura Gaussiana de diez puntos para la función de peso $w(x)=$ $e^{-x}(x+1)$. 


\begin{tabular}{cc}
$\alpha_{n}$ & $\beta_{n}$ \\
\hline $0.187500000000000+01$ & $0.800000000000000+01$ \\
$0.416273584905660+01$ & $0.248437500000000+01$ \\
$0.630804337172262+01$ & $0.701744393022428+01$ \\
$0.839614535739332+01$ & $0.136336834837241+02$ \\
$0.104565831797832+02$ & $0.223064800283651+02$ \\
$0.125014502083910+02$ & $0.330172417706895+02$ \\
$0.145365458691447+02$ & $0.457550498478749+02$ \\
$0.165650187145257+02$ & $0.605132816102859+02$ \\
$0.185887458325360+02$ & $0.772876574238139+02$ \\
$0.206089280316981+02$ & $0.960752379725166+02$ \\
$0.226263760261387+02$ & $0.116873899390755+03$ \\
$0.246416607029748+02$ & $0.139682043889507+03$ \\
$0.266551978406724+02$ & $0.164498430676212+03$ \\
$0.286672987085730+02$ & $0.191322071574788+03$ \\
$0.306782017495408+02$ & $0.220152163381590+03$ \\
$0.326880932093765+02$ & $0.250988042175751+03$ \\
$0.346971210178598+02$ & $0.283829151367034+03$ \\
$0.367054043938972+02$ & $0.318675018690733+03$ \\
$0.387130406540888+02$ & $0.355525239233294+03$ \\
$0.407201101414417+02$ & $0.394379462643979+03$ \\
$0.427266798598730+02$ & $0.435237383325982+03$ \\
$0.447328061987322+02$ & $0.478098732794598+03$ \\
$0.467385370054085+02$ & $0.522963273641436+03$ \\
$0.487439131830141+02$ & $0.569830794708691+03$ \\
$0.507489699368174+02$ & $0.618701107188547+03$ \\
$0.527537377573142+02$ & $0.669574041439138+03$ \\
$0.547582432033577+02$ & $0.722449444362091+03$ \\
$0.567625095317514+02$ & $0.777327177224899+03$ \\
$0.587665572076989+02$ & $0.834207113839075+03$ \\
$0.607704043218979+02$ & $0.893089139025418+03$ \\
$0.627740669338309+02$ & $0.953973147312877+03$ \\
$0.647775593562175+02$ & $0.101685904182892+04$ \\
$0.667808943922009+02$ & $0.108174673334801+04$ \\
$0.687840835342842+02$ & $0.114863613947145+04$ \\
$0.707871371321064+02$ & $0.121752718391711+04$ \\
$0.727900645346715+02$ & $0.128841979590146+04$ \\
$0.747928742115069+02$ & $0.136131390959977+04$ \\
$0.767955738563471+02$ & $0.143620946367257+04$ \\
$0.787981704762473+02$ & $0.151310640084883+04$ \\
$0.808006704684868+02$ & $0.159200466755760+04$
\end{tabular}


-continuación-

\begin{tabular}{cc}
$\alpha_{n}$ & $\beta_{n}$ \\
\hline $0.828030796871955+02$ & $0.167290421360157+04$ \\
$0.848054035012854+02$ & $0.175580499186673+04$ \\
$0.868076468450016+02$ & $0.184070695806343+04$ \\
$0.888098142621765+02$ & $0.192761007049469+04$ \\
$0.908119099450962+02$ & $0.201651428984852+04$ \\
$0.928139377687374+02$ & $0.210741957901108+04$ \\
$0.948159013210121+02$ & $0.220032590289825+04$ \\
$0.968178039295608+02$ & $0.229523322830351+04$ \\
$0.988196486855495+02$ & $0.239214152376013+04$ \\
$0.100821438464858+03$ & $0.249105075941610+04$ \\
$0.102823175946994+03$ & $0.259196090692042+04$ \\
$0.104824863632009+03$ & $0.269487193931949+04$ \\
$0.106826503855676+03$ & $0.279978383096246+04$ \\
$0.108828098803114+03$ & $0.290669655741479+04$ \\
$0.110829650521063+03$ & $0.301561009537888+04$ \\
$0.112831160928958+03$ & $0.312652442262142+04$ \\
$0.114832631828936+03$ & $0.323943951790648+04$ \\
$0.116834064914898+03$ & $0.335435536093396+04$ \\
$0.118835461780732+03$ & $0.347127193228281+04$ \\
$0.120836823927778+03$ & $0.359018921335867+04$ \\
$0.122838152771632+03$ & $0.371110718634541+04$ \\
$0.124839449648342+03$ & $0.383402583416021+04$ \\
$0.126840715820067+03$ & $0.395894514041207+04$ \\
$0.128841952480251+03$ & $0.408586508936311+04$ \\
$0.130843160758361+03$ & $0.421478566589275+04$ \\
$0.132844341724233+03$ & $0.434570685546432+04$ \\
$0.134845496392060+03$ & $0.447862864409393+04$ \\
$0.136846625724059+03$ & $0.461355101832153+04$ \\
$0.138847730633850+03$ & $0.475047396518380+04$ \\
$0.140848811989567+03$ & $0.488939747218885+04$ \\
$0.142849870616728+03$ & $0.503032152729258+04$ \\
$0.144850907300890+03$ & $0.517324611887650+04$ \\
$0.146851922790103+03$ & $0.531817123572697+04$ \\
$0.148852917797184+03$ & $0.546509686701573+04$ \\
$0.150853893001817+03$ & $0.561402300228158+04$ \\
$0.152854849052515+03$ & $0.576494963141321+04$ \\
$0.154855786568430+03$ & $0.591787674463301+04$ \\
$0.156856706141043+03$ & $0.607280433248190+04$ \\
$0.158857608335734+03$ & $0.622973238580490+04$ \\
$0.160858493693244+03$ & $0.638866089573769+04$
\end{tabular}


-continuación-

\begin{tabular}{cc}
$\alpha_{n}$ & $\beta_{n}$ \\
\hline $0.162859362731040+03$ & $0.654958985369382+04$ \\
$0.164860215944587+03$ & $0.671251925135266+04$ \\
$0.166861053808536+03$ & $0.687744908064804+04$ \\
$0.168861876777838+03$ & $0.704437933375747+04$ \\
$0.170862685288781+03$ & $0.721331000309193+04$ \\
$0.172863479759962+03$ & $0.738424108128629+04$ \\
$0.174864260593201+03$ & $0.755717256119011+04$ \\
$0.176865028174397+03$ & $0.773210443585901+04$ \\
$0.178865782874326+03$ & $0.790903669854643+04$ \\
$0.180866525049401+03$ & $0.808796934269587+04$ \\
$0.182867255042371+03$ & $0.826890236193341+04$ \\
$0.184867973182998+03$ & $0.845183575006073+04$ \\
$0.186868679788673+03$ & $0.863676950104837+04$ \\
$0.188869375165014+03$ & $0.882370360902940+04$ \\
$0.190870059606416+03$ & $0.901263806829330+04$ \\
$0.192870733396581+03$ & $0.920357287328021+04$ \\
$0.194871396809008+03$ & $0.939650801857544+04$ \\
$0.196872050107462+03$ & $0.959144349890418+04$ \\
$0.198872693546413+03$ & $0.978837930912652+04$ \\
$0.200873327371458+03$ & $0.998731544423266+04$ \\
$0.202873951819710+03$ & $0.101882518993383+05$ \\
$0.204874567120171+03$ & $0.103911886696805+05$ \\
$0.206875173494090+03$ & $0.105961257506130+05$ \\
$0.208875771155294+03$ & $0.108030631376029+05$ \\
$0.210876360310503+03$ & $0.110120008262263+05$ \\
$0.212876941159638+03$ & $0.112229388121651+05$ \\
$0.214877513896097+03$ & $0.114358770912029+05$ \\
$0.216878078707033+03$ & $0.116508156592224+05$ \\
$0.218878635773609+03$ & $0.118677545122017+05$ \\
$0.220879185271240+03$ & $0.120866936462112+05$ \\
$0.222879727369829+03$ & $0.123076330574109+05$ \\
$0.224880262233985+03$ & $0.125305727420475+05$ \\
$0.226880790023234+03$ & $0.127555126964517+05$ \\
$0.228881310892221+03$ & $0.129824529170355+05$ \\
$0.230881824990896+03$ & $0.132113934002896+05$ \\
$0.232882332464702+03$ & $0.134423341427813+05$ \\
$0.234882833454740+03$ & $0.136752751411520+05$ \\
$0.236883328097942+03$ & $0.139102163921151+05$ \\
$0.238883816527223+03$ & $0.141471578924536+05$ \\
&
\end{tabular}


-continuación-

\begin{tabular}{cc}
$\alpha_{n}$ & $\beta_{n}$ \\
\hline $0.242884775256498+03$ & $0.146270416287256+05$ \\
$0.244885245803563+03$ & $0.148699838585561+05$ \\
$0.246885710631117+03$ & $0.151149263255519+05$ \\
$0.248886169854120+03$ & $0.153618690268158+05$ \\
$0.250886623584320+03$ & $0.156108119595087+05$ \\
$0.252887071930374+03$ & $0.158617551208488+05$ \\
$0.254887514997949+03$ & $0.161146985081091+05$ \\
$0.256887952889834+03$ & $0.163696421186169+05$ \\
$0.258888385706034+03$ & $0.166265859497515+05$ \\
$0.260888813543872+03$ & $0.168855299989430+05$ \\
$0.262889236498077+03$ & $0.171464742636713+05$ \\
$0.264889654660870+03$ & $0.174094187414642+05$ \\
$0.266890068122058+03$ & $0.176743634298966+05$ \\
$0.268890476969102+03$ & $0.179413083265890+05$ \\
$0.270890881287207+03$ & $0.182102534292062+05$ \\
$0.272891281159387+03$ & $0.184811987354567+05$ \\
$0.274891676666545+03$ & $0.187541442430907+05$ \\
$0.276892067887533+03$ & $0.190290899499000+05$ \\
$0.278892454899227+03$ & $0.193060358537161+05$ \\
$0.280892837776584+03$ & $0.195849819524099+05$ \\
$0.282893216592704+03$ & $0.198659282438900+05$ \\
$0.284893591418893+03$ & $0.201488747261024+05$ \\
$0.286893962324714+03$ & $0.204338213970294+05$ \\
$0.288894329378045+03$ & $0.207207682546883+05$ \\
$0.290894692645128+03$ & $0.210097152971312+05$ \\
$0.292895052190622+03$ & $0.213006625224436+05$ \\
$0.294895408077651+03$ & $0.215936099287440+05$ \\
$0.296895760367850+03$ & $0.218885575141828+05$ \\
$0.298896109121408+03$ & $0.221855052769419+05$ \\
$0.300896454397116+03$ & $0.224844532152336+05$
\end{tabular}

Tabla 12.7: Cuadratura Gaussiana de diez puntos para la función de peso $w(x)=$ $e^{-x}\left(x^{2}+3 x+3\right)$. 


\begin{tabular}{cc} 
Abscisas & Pesos \\
\hline $4.334107263780284-01$ & $3.126285312315232+00$ \\
$2.080432318855032+00$ & $3.706170202838551+00$ \\
$4.819002869988582+00$ & $1.092351345744303+00$ \\
$8.864360640960049+00$ & $7.453894181599578-02$ \\
$1.500130120177409+01$ & $6.541972859189739-04$
\end{tabular}

Tabla 12.8: Cuadratura Gaussiana de cinco puntos para la función de peso $w(x)=e^{-x}\left(x^{2}+3 x+3\right)$.

\begin{tabular}{cc} 
Abscisas & Pesos \\
\hline $2.040999954584994-01$ & $1.540405131078210+00$ \\
$1.028387094770998+00$ & $2.845897766236949+00$ \\
$2.410435520337926+00$ & $2.369962904285137+00$ \\
$4.321374767420807+00$ & $1.003385222036850+00$ \\
$6.788153904232589+00$ & $2.162759908385876-01$ \\
$9.876310697774448+00$ & $2.294596348425800-02$ \\
$1.369702559142198+01$ & $1.106205741579204-03$ \\
$1.844094381895615+01$ & $2.070701955826230-05$ \\
$2.447741364509646+01$ & $1.092077619367787-07$ \\
$3.275505137878136+01$ & $7.110886208049865-11$
\end{tabular}

Tabla 12.9: Cuadratura Gaussiana de diez puntos para la función de peso $w(x)=$ $e^{-x}\left(x^{2}+3 x+3\right)$. 


\begin{tabular}{cc}
$\alpha_{n}$ & $\beta_{n}$ \\
\hline $0.218750000000000+01$ & $0.480000000000000+02$ \\
$0.461687424058323+01$ & $0.321484375000000+01$ \\
$0.684097433647064+01$ & $0.844654222689417+01$ \\
$0.897907268480269+01$ & $0.158091158709766+02$ \\
$0.110752589635655+02$ & $0.252613627146857+02$ \\
$0.131476069761617+02$ & $0.367732373487596+02$ \\
$0.152047965944759+02$ & $0.503277286677345+02$ \\
$0.172515798299534+02$ & $0.659147478318503+02$ \\
$0.192908233617017+02$ & $0.835277908123652+02$ \\
$0.213243832328397+02$ & $0.103162348393842+03$ \\
$0.233535261073412+02$ & $0.124815118564845+03$ \\
$0.253791521837523+02$ & $0.148483584512177+03$ \\
$0.274019221849538+02$ & $0.174165769584679+03$ \\
$0.294223340221210+02$ & $0.201860084872488+03$ \\
$0.314407712749077+02$ & $0.231565229057218+03$ \\
$0.334575350344164+02$ & $0.263280119736306+03$ \\
$0.354728654917744+02$ & $0.297003844719389+03$ \\
$0.374869569744102+02$ & $0.332735626538171+03$ \\
$0.394999686653239+02$ & $0.370474795996244+03$ \\
$0.415120324018612+02$ & $0.410220772075637+03$ \\
$0.435232584525358+02$ & $0.451973046417579+03$ \\
$0.455337398650990+02$ & $0.495731171160502+03$ \\
$0.475435557864809+02$ & $0.541494749285090+03$ \\
$0.495527740307023+02$ & $0.589263426860321+03$ \\
$0.515614530885360+02$ & $0.639036886750866+03$ \\
$0.535696437171720+02$ & $0.690814843461841+03$ \\
$0.555773902100199+02$ & $0.744597038878725+03$ \\
$0.575847314201595+02$ & $0.800383238719080+03$ \\
$0.595917015920975+02$ & $0.858173229555600+03$ \\
$0.615983310429328+02$ & $0.917966816301714+03$ \\
$0.636046467241764+02$ & $0.979763820074663+03$ \\
$0.656106726882100+02$ & $0.104356407636890+04$ \\
$0.676164304779692+02$ & $0.110936743348640+04$ \\
$0.696219394543699+02$ & $0.117717375118092+04$ \\
$0.716272170729164+02$ & $0.124698289948163+04$ \\
$0.736322791185692+02$ & $0.131879475766788+04$ \\
$0.756371399061255+02$ & $0.139260921337202+04$ \\
$0.776418124519491+02$ & $0.146842616179110+04$ \\
$0.796463086217729+02$ & $0.154624550499197+04$ \\
$0.816506392584223+02$ & $0.162606715129629+04$
\end{tabular}


-continuación-

\begin{tabular}{cc}
$\alpha_{n}$ & $\beta_{n}$ \\
\hline $0.836548142926069+02$ & $0.170789101473471+04$ \\
$0.856588428393752+02$ & $0.179171701456082+04$ \\
$0.876627332823754+02$ & $0.187754507481712+04$ \\
$0.896664933477058+02$ & $0.196537512394626+04$ \\
$0.916701301688416+02$ & $0.205520709444202+04$ \\
$0.936736503438882+02$ & $0.214704092253511+04$ \\
$0.956770599862082+02$ & $0.224087654790964+04$ \\
$0.976803647693141+02$ & $0.233671391344668+04$ \\
$0.996835699667758+02$ & $0.243455296499182+04$ \\
$0.101686680487788+03$ & $0.253439365114399+04$ \\
$0.103689700908941+03$ & $0.263623592306330+04$ \\
$0.105692635502671+03$ & $0.274007973429577+04$ \\
$0.107695488262787+03$ & $0.284592504061318+04$ \\
$0.109698262927422+03$ & $0.295377179986654+04$ \\
$0.111700962999721+03$ & $0.306361997185164+04$ \\
$0.113703591766511+03$ & $0.317546951818571+04$ \\
$0.115706152315191+03$ & $0.328932040219387+04$ \\
$0.117708647549037+03$ & $0.340517258880452+04$ \\
$0.119711080201092+03$ & $0.352302604445292+04$ \\
$0.121713452846798+03$ & $0.364288073699200+04$ \\
$0.123715767915500+03$ & $0.376473663560993+04$ \\
$0.125718027700939+03$ & $0.388859371075368+04$ \\
$0.127720234370836+03$ & $0.401445193405816+04$ \\
$0.129722389975664+03$ & $0.414231127828044+04$ \\
$0.131724496456683+03$ & $0.427217171723849+04$ \\
$0.133726555653309+03$ & $0.440403322575424+04$ \\
$0.135728569309888+03$ & $0.453789577960049+04$ \\
$0.137730539081925+03$ & $0.467375935545135+04$ \\
$0.139732466541825+03$ & $0.481162393083598+04$ \\
$0.141734353184176+03$ & $0.495148948409530+04$ \\
$0.143736200430640+03$ & $0.509335599434158+04$ \\
$0.145738009634467+03$ & $0.523722344142043+04$ \\
$0.147739782084670+03$ & $0.538309180587529+04$ \\
$0.149741519009898+03$ & $0.553096106891407+04$ \\
$0.151743221582020+03$ & $0.568083121237776+04$ \\
$0.153744890919463+03$ & $0.583270221871097+04$ \\
$0.155746528090302+03$ & $0.598657407093423+04$ \\
$0.157748134115138+03$ & $0.614244675261785+04$ \\
$0.159749709969784+03$ & $0.630032024785732+04$ \\
$0.161751256587755+03$ & $0.646019454125008+04$
\end{tabular}


-continuación-

\begin{tabular}{cc}
$\alpha_{n}$ & $\beta_{n}$ \\
\hline $0.163752774862603+03$ & $0.662206961787366+04$ \\
$0.165754265650088+03$ & $0.678594546326494+04$ \\
$0.167755729770212+03$ & $0.695182206340058+04$ \\
$0.169757168009114+03$ & $0.711969940467859+04$ \\
$0.171758581120853+03$ & $0.728957747390073+04$ \\
$0.173759969829068+03$ & $0.746145625825599+04$ \\
$0.175761334828541+03$ & $0.763533574530481+04$ \\
$0.177762676786660+03$ & $0.781121592296423+04$ \\
$0.179763996344792+03$ & $0.798909677949370+04$ \\
$0.181765294119575+03$ & $0.816897830348163+04$ \\
$0.183766570704129+03$ & $0.835086048383270+04$ \\
$0.185767826669200+03$ & $0.853474330975562+04$ \\
$0.187769062564231+03$ & $0.872062677075168+04$ \\
$0.189770278918376+03$ & $0.890851085660371+04$ \\
$0.191771476241452+03$ & $0.909839555736563+04$ \\
$0.193772655024841+03$ & $0.929028086335252+04$ \\
$0.195773815742335+03$ & $0.948416676513105+04$ \\
$0.197774958850940+03$ & $0.968005325351048+04$ \\
$0.199776084791635+03$ & $0.987794031953399+04$ \\
$0.201777193990083+03$ & $0.100778279544704+05$ \\
$0.203778286857312+03$ & $0.102797161498065+05$ \\
$0.205779363790354+03$ & $0.104836048972390+05$ \\
$0.207780425172855+03$ & $0.106894941886680+05$ \\
$0.209781471375646+03$ & $0.108973840161895+05$ \\
$0.211782502757290+03$ & $0.111072743720894+05$ \\
$0.213783519664600+03$ & $0.113191652488366+05$ \\
$0.215784522433130+03$ & $0.115330566390773+05$ \\
$0.217785511387637+03$ & $0.117489485356292+05$ \\
$0.219786486842529+03$ & $0.119668409314759+05$ \\
$0.221787449102280+03$ & $0.121867338197617+05$ \\
$0.223788398461836+03$ & $0.124086271937861+05$ \\
$0.225789335206988+03$ & $0.126325210469995+05$ \\
$0.227790259614741+03$ & $0.128584153729980+05$ \\
$0.229791171953655+03$ & $0.130863101655192+05$ \\
$0.231792072484173+03$ & $0.133162054184378+05$ \\
$0.233792961458934+03$ & $0.135481011257611+05$ \\
$0.235793839123073+03$ & $0.137819972816257+05$ \\
$0.237794705714503+03$ & $0.140178938802930+05$ \\
$0.239795561464188+03$ & $0.142557909161457+05$ \\
&
\end{tabular}


-continuación-

\begin{tabular}{cc}
$\alpha_{n}$ & $\beta_{n}$ \\
\hline $0.243797241328966+03$ & $0.147375862775242+05$ \\
$0.245798065873511+03$ & $0.149814845923908+05$ \\
$0.247798880435673+03$ & $0.152273833231182+05$ \\
$0.249799685215326+03$ & $0.154752824646450+05$ \\
$0.251800480406784+03$ & $0.157251820120117+05$ \\
$0.253801266198998+03$ & $0.159770819603578+05$ \\
$0.255802042775745+03$ & $0.162309823049190+05$ \\
$0.257802810315811+03$ & $0.164868830410247+05$ \\
$0.259803568993159+03$ & $0.167447841640953+05$ \\
$0.261804318977102+03$ & $0.170046856696399+05$ \\
$0.263805060432455+03$ & $0.172665875532536+05$ \\
$0.265805793519690+03$ & $0.175304898106156+05$ \\
$0.267806518395085+03$ & $0.177963924374867+05$ \\
$0.269807235210858+03$ & $0.180642954297073+05$ \\
$0.271807944115308+03$ & $0.183341987831951+05$ \\
$0.273808645252939+03$ & $0.186061024939436+05$ \\
$0.275809338764585+03$ & $0.188800065580194+05$ \\
$0.277810024787532+03$ & $0.191559109715611+05$ \\
$0.279810703455627+03$ & $0.194338157307770+05$ \\
$0.281811374899395+03$ & $0.197137208319434+05$ \\
$0.283812039246135+03$ & $0.199956262714031+05$ \\
$0.285812696620030+03$ & $0.202795320455636+05$ \\
$0.287813347142241+03$ & $0.205654381508956+05$ \\
$0.289813990931001+03$ & $0.208533445839312+05$ \\
$0.291814628101706+03$ & $0.211432513412628+05$ \\
$0.293815258767000+03$ & $0.214351584195414+05$ \\
$0.295815883036863+03$ & $0.217290658154753+05$ \\
$0.297816501018686+03$ & $0.220249735258285+05$ \\
$0.299817112817354+03$ & $0.223228815474199+05$ \\
$0.301817718535317+03$ & $0.226227898771214+05$
\end{tabular}

Tabla 12.10: Cuadratura Gaussiana de diez puntos para la función de peso $w(x)=e^{-x}\left(x^{3}+6 x^{2}+15 x+15\right)$. 


\begin{tabular}{cc} 
Abscisas & Pesos \\
\hline $4.856397204365821-01$ & $1.767567198376409+01$ \\
$2.322943622519654+00$ & $2.242442391649330+01$ \\
$5.300917914775939+00$ & $7.337666788536437+00$ \\
$9.594450731980713+00$ & $5.568334586622845-01$ \\
$1.599572823570913+01$ & $5.403852543884571-03$
\end{tabular}

Tabla 12.11: Cuadratura Gaussiana de cinco puntos para la función de peso $w(x)=e^{-x}\left(x^{3}+6 x^{2}+15 x+15\right)$.

\begin{tabular}{cc} 
Abscisas & Pesos \\
\hline $2.231269688967887-01$ & $8.448330854095689+00$ \\
$1.126944113234777+00$ & $1.619852066263472+01$ \\
$2.631956961477349+00$ & $1.463539994003167+01$ \\
$4.682584404835726+00$ & $6.870558759754008+00$ \\
$7.292762485612102+00$ & $1.644418987882815+00$ \\
$1.052419789528722+01$ & $1.924194238741014-01$ \\
$1.448797342967301+01$ & $1.014413253898122-02$ \\
$1.937659688426222+01$ & $2.060654091398202-04$ \\
$2.556367111733745+01$ & $1.172955323584843-06$ \\
$3.400905595993764+01$ & $8.235547686627145-10$
\end{tabular}

Tabla 12.12: Cuadratura Gaussiana de diez puntos para la función de peso $w(x)=e^{-x}\left(x^{3}+6 x^{2}+15 x+15\right)$. 


\begin{tabular}{cc}
$\alpha_{n}$ & $\beta_{n}$ \\
\hline $0.246093750000000+01$ & $0.384000000000000+03$ \\
$0.502710707169388+01$ & $0.394378662109375+01$ \\
$0.732831797310007+01$ & $0.985567614999046+01$ \\
$0.951654159603280+01$ & $0.179456776682826+02$ \\
$0.116493835633401+02$ & $0.281570846181982+02$ \\
$0.137503753782961+02$ & $0.404489959818953+02$ \\
$0.158308665389149+02$ & $0.547989994939242+02$ \\
$0.178971352534647+02$ & $0.711939054990667+02$ \\
$0.199530116690086+02$ & $0.896251310902562+02$ \\
$0.220010001087101+02$ & $0.110086640551224+03$ \\
$0.240428235362128+02$ & $0.132573956224636+03$ \\
$0.260797146304880+02$ & $0.157083629627076+03$ \\
$0.281125832530329+02$ & $0.183612930328100+03$ \\
$0.301421184065812+02$ & $0.212159649165508+03$ \\
$0.321688531646057+02$ & $0.242721967010396+03$ \\
$0.341932075698882+02$ & $0.275298363715294+03$ \\
$0.362155178657620+02$ & $0.309887552956916+03$ \\
$0.382360569464403+02$ & $0.346488434416856+03$ \\
$0.402550489946184+02$ & $0.385100057931781+03$ \\
$0.422726801701718+02$ & $0.425721596119981+03$ \\
$0.442891065544624+02$ & $0.468352323142666+03$ \\
$0.463044601485887+02$ & $0.512991597990447+03$ \\
$0.483188534666824+02$ & $0.559638851164179+03$ \\
$0.503323830984120+02$ & $0.608293573940291+03$ \\
$0.523451325041175+02$ & $0.658955309630533+03$ \\
$0.543571742310717+02$ & $0.711623646399556+03$ \\
$0.563685716877644+02$ & $0.766298211312755+03$ \\
$0.583793805769791+02$ & $0.822978665365470+03$ \\
$0.603896500627664+02$ & $0.881664699302238+03$ \\
$0.623994237279288+02$ & $0.942356030077444+03$ \\
$0.644087403651489+02$ & $0.100505239784080+04$ \\
$0.664176346349416+02$ & $0.106975356335535+04$ \\
$0.684261376161878+02$ & $0.113645930577433+04$ \\
$0.704342772694171+02$ & $0.120516942071763+04$ \\
$0.724420788287523+02$ & $0.127588371859995+04$ \\
$0.744495651351664+02$ & $0.134860202317125+04$ \\
$0.764567569211790+02$ & $0.142332417023768+04$ \\
$0.784636730551529+02$ & $0.150005000653614+04$ \\
$0.804703307518041+02$ & $0.157877938874069+04$ \\
$0.824767457543234+02$ & $0.165951218258220+04$
\end{tabular}


-continuación-

\begin{tabular}{cc}
$\alpha_{n}$ & $\beta_{n}$ \\
\hline $0.844829324925283+02$ & $0.174224826206599+04$ \\
$0.864889042206952+02$ & $0.182698750877429+04$ \\
$0.884946731380882+02$ & $0.191372981124273+04$ \\
$0.905002504946994+02$ & $0.200247506440120+04$ \\
$0.925056466843005+02$ & $0.209322316907139+04$ \\
$0.945108713265684+02$ & $0.218597403151398+04$ \\
$0.965159333397723+02$ & $0.228072756301966+04$ \\
$0.985208410052781+02$ & $0.237748367953889+04$ \\
$0.100525602024939+03$ & $0.247624230134600+04$ \\
$0.102530223572284+03$ & $0.257700335273382+04$ \\
$0.104534712338274+03$ & $0.267976676173552+04$ \\
$0.106539074572309+03$ & $0.278453245987076+04$ \\
$0.108543316119046+03$ & $0.289130038191349+04$ \\
$0.110547442451527+03$ & $0.300007046567943+04$ \\
$0.112551458701052+03$ & $0.311084265183093+04$ \\
$0.114555369684166+03$ & $0.322361688369773+04$ \\
$0.116559179927081+03$ & $0.333839310711198+04$ \\
$0.118562893687819+03$ & $0.345517127025610+04$ \\
$0.120566514976328+03$ & $0.357395132352242+04$ \\
$0.122570047572775+03$ & $0.369473321938333+04$ \\
$0.124573495044222+03$ & $0.381751691227119+04$ \\
$0.126576860759841+03$ & $0.394230235846690+04$ \\
$0.128580147904823+03$ & $0.406908951599657+04$ \\
$0.130583359493110+03$ & $0.419787834453549+04$ \\
$0.132586498379061+03$ & $0.432866880531873+04$ \\
$0.134589567268167+03$ & $0.446146086105799+04$ \\
$0.136592568726894+03$ & $0.459625447586395+04$ \\
$0.138595505191742+03$ & $0.473304961517387+04$ \\
$0.140598378977597+03$ & $0.487184624568392+04$ \\
$0.142601192285427+03$ & $0.501264433528587+04$ \\
$0.144603947209402+03$ & $0.515544385300783+04$ \\
$0.146606645743463+03$ & $0.530024476895874+04$ \\
$0.148609289787421+03$ & $0.544704705427621+04$ \\
$0.150611881152587+03$ & $0.559585068107770+04$ \\
$0.152614421567019+03$ & $0.574665562241449+04$ \\
$0.154616912680371+03$ & $0.589946185222851+04$ \\
$0.156619356068420+03$ & $0.605426934531160+04$ \\
$0.158621753237262+03$ & $0.621107807726725+04$ \\
$0.160624105627233+03$ & $0.636988802447443+04$ \\
$0.162626414616551+03$ & $0.653069916405351+04$
\end{tabular}


-continuación-

\begin{tabular}{cc}
$\alpha_{n}$ & $\beta_{n}$ \\
\hline $0.164628681524729+03$ & $0.669351147383409+04$ \\
$0.166630907615745+03$ & $0.685832493232456+04$ \\
$0.168633094101020+03$ & $0.702513951868339+04$ \\
$0.170635242142192+03$ & $0.719395521269190+04$ \\
$0.172637352853721+03$ & $0.736477199472849+04$ \\
$0.174639427305323+03$ & $0.753758984574426+04$ \\
$0.176641466524255+03$ & $0.771240874723987+04$ \\
$0.178643471497462+03$ & $0.788922868124357+04$ \\
$0.180645443173586+03$ & $0.806804963029040+04$ \\
$0.182647382464858+03$ & $0.824887157740238+04$ \\
$0.184649290248877+03$ & $0.843169450606969+04$ \\
$0.186651167370284+03$ & $0.861651840023284+04$ \\
$0.188653014642337+03$ & $0.880334324426561+04$ \\
$0.190654832848394+03$ & $0.899216902295887+04$ \\
$0.192656622743312+03$ & $0.918299572150517+04$ \\
$0.194658385054770+03$ & $0.937582332548401+04$ \\
$0.196660120484513+03$ & $0.957065182084787+04$ \\
$0.198661829709530+03$ & $0.976748119390880+04$ \\
$0.200663513383164+03$ & $0.996631143132568+04$ \\
$0.202665172136167+03$ & $0.101671425200920+05$ \\
$0.204666806577693+03$ & $0.103699744475244+05$ \\
$0.206668417296239+03$ & $0.105748072012512+05$ \\
$0.208670004860541+03$ & $0.107816407692022+05$ \\
$0.210671569820416+03$ & $0.109904751395980+05$ \\
$0.212673112707568+03$ & $0.112013103009408+05$ \\
$0.214674634036341+03$ & $0.114141462420047+05$ \\
$0.216676134304451+03$ & $0.116289829518269+05$ \\
$0.218677613993662+03$ & $0.118458204196993+05$ \\
$0.220679073570445+03$ & $0.120646586351599+05$ \\
$0.222680513486591+03$ & $0.122854975879853+05$ \\
$0.224681934179805+03$ & $0.125083372681831+05$ \\
$0.226683336074261+03$ & $0.127331776659846+05$ \\
$0.228684719581140+03$ & $0.129600187718376+05$ \\
$0.230686085099134+03$ & $0.131888605764003+05$ \\
$0.232687433014932+03$ & $0.134197030705344+05$ \\
$0.234688763703678+03$ & $0.136525462452992+05$ \\
$0.236690077529414+03$ & $0.138873900919453+05$ \\
$0.238691374845497+03$ & $0.141242346019096+05$ \\
$0.240692655994999+03$ & $0.143630797668091+05$ \\
$0.242693921311091+03$ & $0.146039255784359+05$
\end{tabular}


-continuación-

\begin{tabular}{cc}
$\alpha_{n}$ & -continuacion- \\
\hline $0.244695171117403+03$ & $0.148467720287524+05$ \\
$0.246696405728378+03$ & $0.150916191098858+05$ \\
$0.248697625449600+03$ & $0.153384668141240+05$ \\
$0.250698830578118+03$ & $0.155873151339106+05$ \\
$0.252700021402742+03$ & $0.158381640618410+05$ \\
$0.254701198204343+03$ & $0.160910135906575+05$ \\
$0.256702361256127+03$ & $0.163458637132460+05$ \\
$0.258703510823902+03$ & $0.166027144226315+05$ \\
$0.260704647166335+03$ & $0.168615657119744+05$ \\
$0.262705770535197+03$ & $0.171224175745673+05$ \\
$0.264706881175597+03$ & $0.173852700038308+05$ \\
$0.266707979326205+03$ & $0.176501229933106+05$ \\
$0.268709065219472+03$ & $0.179169765366739+05$ \\
$0.270710139081835+03$ & $0.181858306277063+05$ \\
$0.272711201133914+03$ & $0.184566852603089+05$ \\
$0.274712251590706+03$ & $0.187295404284952+05$ \\
$0.276713290661765+03$ & $0.190043961263879+05$ \\
$0.278714318551379+03$ & $0.192812523482167+05$ \\
$0.280715335458741+03$ & $0.195601090883152+05$ \\
$0.282716341578108+03$ & $0.198409663411186+05$ \\
$0.284717337098958+03$ & $0.201238241011606+05$ \\
$0.286718322206143+03$ & $0.204086823630718+05$ \\
$0.288719297080029+03$ & $0.206955411215765+05$ \\
$0.290720261896639+03$ & $0.209844003714911+05$ \\
$0.292721216827783+03$ & $0.212752601077213+05$ \\
$0.294722162041189+03$ & $0.215681203252604+05$ \\
$0.296723097700628+03$ & $0.218629810191870+05$ \\
$0.298724023966029+03$ & $0.221598421846630+05$ \\
$0.300724940993599+03$ & $0.224587038169316+05$ \\
$0.302725848935929+03$ & $0.227595659113154+05$
\end{tabular}

Tabla 12.13: Cuadratura Gaussiana de diez puntos para la función de peso $w(x)=e^{-x}\left(x^{4}+10 x^{3}+45 x^{2}+105 x+105\right)$. 


\begin{tabular}{cc} 
Abscisas & Pesos \\
\hline $5.305812861509350-01$ & $1.358220332619339+02$ \\
$2.536207092935022+00$ & $1.796388609811520+02$ \\
$5.735844745506172+00$ & $6.327381970652042+01$ \\
$1.026350610283796+01$ & $5.210433302655349+00$ \\
$1.691614847673673+01$ & $5.485274773833875-02$
\end{tabular}

Tabla 12.14: Cuadratura Gaussiana de cinco puntos para la función de peso $w(x)=e^{-x}\left(x^{4}+10 x^{3}+45 x^{2}+105 x+105\right)$.

\begin{tabular}{cc} 
Abscisas & Pesos \\
\hline $2.394753416728400-01$ & $6.357953668602757+01$ \\
$1.211845745033918+00$ & $1.244506182881016+02$ \\
$2.827067796266381+00$ & $1.184521571016887+02$ \\
$5.007615713168725+00$ & $5.988971602953811+01$ \\
$7.753735215036700+00$ & $1.554442963337607+01$ \\
$1.112224268436729+01$ & $1.969218801386250+00$ \\
$1.522357277696554+01$ & $1.118716465283256-01$ \\
$2.025180986588678+01$ & $2.436979062283901-03$ \\
$2.658459772810010+01$ & $1.482316758208445-05$ \\
$3.519271378606304+01$ & $1.112352424440037-08$
\end{tabular}

Tabla 12.15: Cuadratura Gaussiana de diez puntos para la función de peso $w(x)=e^{-x}\left(x^{4}+10 x^{3}+45 x^{2}+105 x+105\right)$. 


\section{Apéndice D}

Se presentan las gráficas correspondientes a los polinomios ortogonales con respecto a funciones de peso $w(x)=e^{-x}$ que es de Laguerre, $w(x)=e^{-x}(x+$ $1), w(x)=e^{-x}\left(x^{2}+3 x+3\right)$ у $w(x)=e^{-x}\left(x^{3}+6 x^{2}+15 x+15\right)$.

\subsection{Gráficas de polinomios ortogonales para $w(x)=$} $e^{-x} \Theta_{l}(x)$.
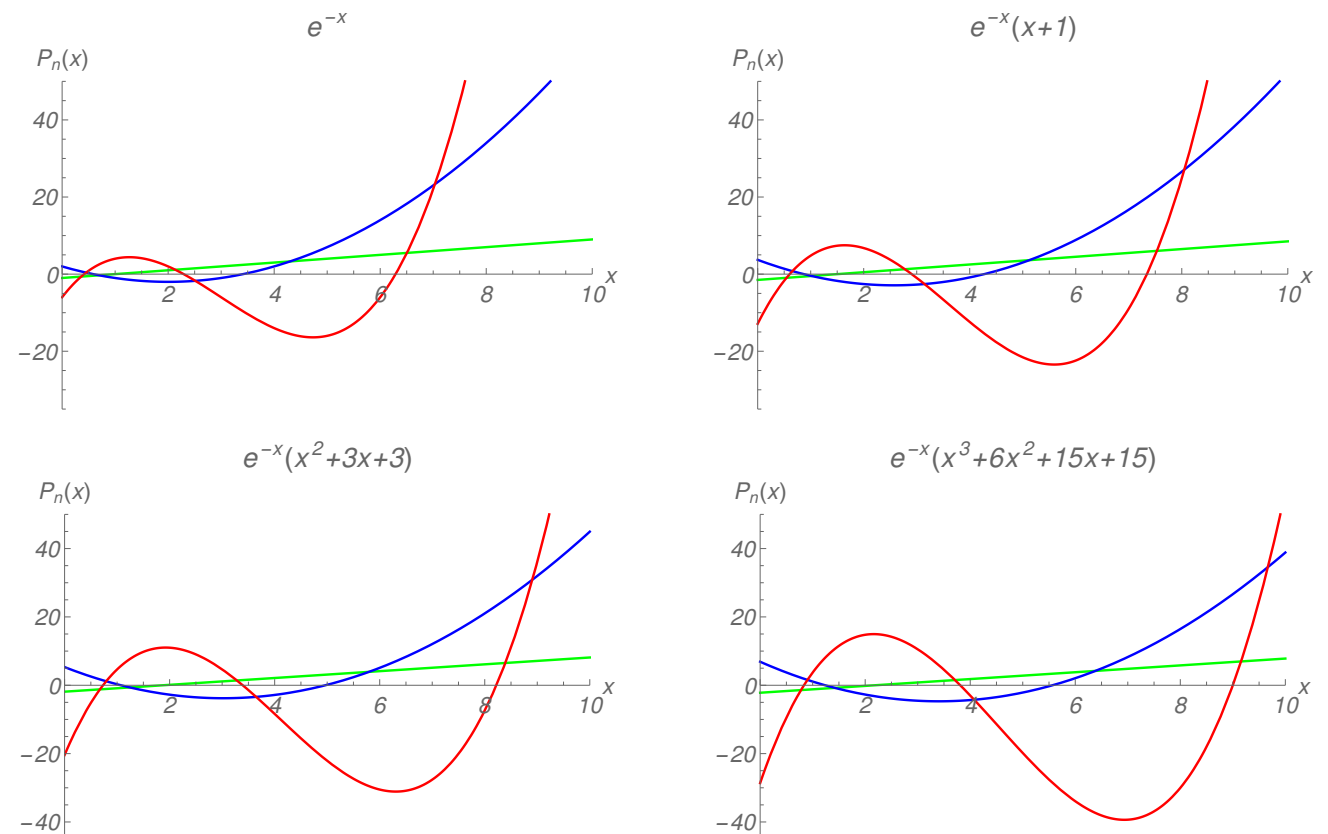

Figura 13.1: Gráficas de los tres primeros polinomios ortogonales en el intervalo $0 \leq x \leq 10$. El polinomio de primer grado $P_{1}(x)$ (verde), el polinomio de segundo grado $P_{2}(x)$ (azul) y el polinomio de tercer grado $P_{3}(x)$ (rojo). 


\section{Apéndice $\mathrm{E}$}

Se presentan las gráficas correspondientes a las funciones de peso para la transformada de Hankel $w(x)=K_{0}(x), w(x)=x K_{1}(x), w(x)=x^{2} K_{2}(x)$ y $w(x)=x^{3} K_{3}(x)$.

14.1 Gráficas de las funciones de peso $w(x)=x^{m} K_{m}(x)$.



Figura 14.1: Gráficas de las funciones de peso correspondientes a la transformada de Hankel en el intervalo $0 \leq x \leq 8$. 
Se presentan las gráficas correspondientes a las funciones de peso para la transformada de Bessel esférica $w(x)=e^{-x} \Theta_{0}, w(x)=e^{-x} \Theta_{1}, w(x)=e^{-x} \Theta_{2}$ y $w(x)=e^{-x} \Theta_{3}$.

\subsection{Gráficas de las funciones de peso $w(x)=e^{-x} \Theta_{l}(x)$.}



Figura 14.2: Gráficas de las funciones de peso correspondientes a la transformada de Bessel esférica en el intervalo $0 \leq x \leq 8$. 


\section{Apéndice $\mathrm{F}$}

A través del tratamiento de cuadratura Gaussiana se plantea la solución en términos de un problema de álgebra lineal por lo que definimos la integral (4.0.7) como la aproximación

$$
\int_{0}^{\infty} f(x) w(x) \mathrm{d} x \approx \sum_{k=1}^{N} f\left(x_{k}\right) w_{k}
$$

donde las $x_{k}$ corresponden a los nodos y las $w_{k}$ a los pesos de la cuadratura Gaussiana para $N$ puntos. Esta formulación del problema corresponde a un sistema de ecuaciones no lineales de tamaño $2 N \times 2 N$, respecto a una base polinomial $\mathbb{P}_{n}=\left\{1, x, x^{2}, x^{3}, \ldots\right\}$, tal que $\operatorname{dim} \mathbb{P}_{n}=2 N$. La construcción de cada una de las ecuaciones no lineales a partir de (15.0.1) se define imponiendo la condición de que $f(x)$ sea exacta para cada elemento de la base, así al tomar sucesivamente un nuevo elemento de $\mathbb{P}_{n}$ se construye una ecuación diferente. Lo anterior se hace considerando la función de peso $w(x)$ particular en este caso de acuerdo a la integral (4.0.7) tenemos que $w(x)=J_{\nu}(x) e^{-x}$. La introducción del término $e^{-x}$ en la función de peso es para asegurar la convergencia de las integrales $\int_{0}^{\infty} x^{n} J_{\nu}(x) e^{-x} \mathrm{~d} x$.

La construcción general para el sistema de $2 N$ ecuaciones no lineales con $2 N$ incógnitas $\left\{x_{1}, x_{2}, \ldots, x_{N}\right\}$ y $\left\{w_{1}, w_{2}, \ldots, w_{N}\right\}$ está dada por

$$
\begin{aligned}
w_{1}+w_{2}+w_{3}+\cdots+w_{N} & =\int_{0}^{\infty} J_{\nu}(x) e^{-x} \mathrm{~d} x \\
x_{1} w_{1}+x_{2} w_{2}+x_{3} w_{3}+\cdots+x_{N} w_{N} & =\int_{0}^{\infty} x J_{\nu}(x) e^{-x} \mathrm{~d} x \\
x_{1}^{2} w_{1}+x_{2}^{2} w_{2}+x_{3}^{2} w_{3}+\cdots+x_{N}^{2} w_{N} & =\int_{0}^{\infty} x^{2} J_{\nu}(x) e^{-x} \mathrm{~d} x
\end{aligned}
$$

se continúa así hasta completar la base $\mathbb{P}_{n}$

$$
x_{1}^{2 N-1} w_{1}+x_{2}^{2 N-1} w_{2}+x_{3}^{2 N-1} w_{3}+\cdots+x_{N}^{2 N-1} w_{N}=\int_{0}^{\infty} x^{2 N-1} J_{\nu}(x) e^{-x} \mathrm{~d} x .
$$

Las integrales que aparecen en el lado derecho del sistema de ecuaciones no lineales corresponden a los momentos generados por la función de peso $w(x)$ y la base $\mathbb{P}_{n}$ mediante $\int_{0}^{\infty} x^{n} J_{\nu}(x) e^{-x} \mathrm{~d} x$ que tiene solución analítica.

Considerando funciones de Bessel de primer tipo con $\nu=0$ se probó la aplicación de esta metodología para calcular las cuadraturas Gaussianas ya 
que para algunos momentos se tiene que $\int_{0}^{\infty} x^{n} J_{0}(x) e^{-x} \mathrm{~d} x<0$. Usando el paquete Mathematica versión 10.0 [78] se resolvieron los sistemas de ecuaciones no lineales. A continuación se presentan los valores de pesos $\left\{x_{j}\right\}_{1}^{4}$ y nodos $\left\{w_{j}\right\}_{1}^{4}$ de la cuadratura Gaussiana de cuatro puntos

\begin{tabular}{cc} 
Nodos & Pesos \\
\hline $0.443744+01+i 0.128721+01$ & $-0.321954-02+i 0.900534-03$ \\
$0.443744+01-i 0.128721+01$ & $-0.321954-02-i 0.900534-03$ \\
$0.949345+00$ & $0.334834+00$ \\
$0.175784+00$ & $0.378711+00$
\end{tabular}

Tabla 15.1: Cuadratura Gaussiana $N=4$ y función de peso $w(x)=J_{0}(x) e^{-x}$.

Para estos sistemas no lineales las soluciones, es decir nodos y pesos pueden ser valores complejos $\left\{x_{k}, w_{k}\right\}_{1}^{N} \in \mathbb{C}$. Sin embargo para $N=5$ se tiene que las soluciones son valores reales, además encontramos valores negativos, lo cual indica que los nodos se encuentran fuera del intervalo de integración. En la Tabla (14.2) se muestra la aplicación de estas nuevas cuadraturas generadas con $N=4$ para calcular integrales del tipo (4.0.7).

\begin{tabular}{ccc}
$\rho$ & $f_{1}(x)=\frac{1}{x+1}$ & $f_{2}(x)=\frac{1}{x^{2}+1}$ \\
\hline 0.5 & 1.184497 & 1.156487 \\
& 1.184714 & 1.167650 \\
2.0 & 0.440581 & 0.537450 \\
& 0.441931 & 0.537908 \\
10.0 & $9.907407 \cdot 10^{-2}$ & 0.101126 \\
& $9.993135 \cdot 10^{-2}$ & 0.101924 \\
20.0 & $4.987765 \cdot 10^{-2}$ & $5.012801 \cdot 10^{-2}$ \\
& $5.046292 \cdot 10^{-2}$ & $5.083596 \cdot 10^{-2}$ \\
30.0 & $3.329665 \cdot 10^{-2}$ & $3.337075 \cdot 10^{-2}$ \\
& $3.374011 \cdot 10^{-2}$ & $3.391081 \cdot 10^{-2}$
\end{tabular}

Tabla 15.2: Resultados de la regla de cuadratura aplicados a la integral (4.0.7). La primera entrada muestra el resultado analítico mientras que la segunda entrada es el resultado numérico.

Mediante el paquete Mathematica versión 10.0 no se pudo resolver sistemas de ecuaciones no lineales para $N>5$, ya que el programa no fue capaz de dar una solución para $N=6$. No obstante, uno espera mejorar la 
aproximación incrementando el orden de la cuadratura resolviendo sistemas de ecuaciones no lineales más grandes. 


\section{Bibliografía}

[1] F. London, On the Bose-Einstein condensation, Phys. Rev. 54 (1938) 947. doi:10.1103/PhysRev. 54.947.

[2] P. Jordan, Über eine neue Begründung der Quantenmechanik, Z. Physik 40 (1927) 809-838. doi:10.1007/BF01390903.

[3] I. N. Sneddon, Fourier Transforms, Dover, Publication, Inc., New York, (1995) Ch. 1, Ch. 2 y Ch. 8.

[4] I. McCarthy, E. Weigold, (e, 2e) spectroscopy, Phys. Rep. 27 (1976) 275-371. doi:10.1016/0370-1573(76)90005-3.

[5] L. Pauling, The nature of the chemical bond, 3rd Ed., Cornell University, New York, (1960).

[6] P. Coppens, M. B. Hall, Electron distributions and the chemical bond, Plenum, New York, (1982).

[7] R. K. Pathak, B. S. Sharma, A. J. Thakkar, Approximate relationships between density power integrals, moments of the momentum density, and interelectronic repulsions in diatomic molecules, J. Chem. Phys. 85 (1986) 958. doi:dx.doi.org/10.1063/1.451252.

[8] H. A. Bethe, E. E. Salpeter, Quantum Mechanics of one- and two-electron atoms, Springer-Verlag, Berlin, (1957) p. 170.

[9] A. J. Thakkar, The momentum density perspective of the electronic structure of atoms and molecules, Adv. Chem. Phys. 28 (2004) 303-352.

[10] C. Cohen-Tannoudji, B. Diu, F. Laloë, Quantum Mechanics, John Willey \& Sons, Paris, (1973) Vol. I. Ch. 2.

[11] K. Gottfried, Quantum Mechanics, W. A. Benjamin Inc., USA, (1966) Vol. I. Ch. 2.

[12] P. A. M. Dirac, The Principles of Quantum Mechanics, 3rd Ed., Oxford, New York, (1947) 103.

[13] R. N. Bracewell, The Fourier Transform and its Applications, Mc Graw Hill, USA, (2000) Ch. 13. 
[14] R. Piessens, The Transforms and Applications Handbook, 2nd ed., Ed. A.D. Poularikis, (CRC Press LLC, Boca Raton, 2000) Ch. 9.

[15] H. Laguna G., Correlación en los modelos de una y dos partículas en una caja, UAM-Iztapalapa, (2011).

[16] E. M. Stein, G. Weiss, Introduction to Fourier analysis on Euclidean spaces, Princeton, New Jersey, New Jersey, (1975) Ch. 4.

[17] B. Podolsky, L. Pauling, The momentum distribution in hydrogen-like atoms, Phys. Rev. 34 (1929) 109-116. doi:10.1103/PhysRev.34.109.

[18] P. Kaijser, V. H. Smith Jr., Evaluation of momentum distributions and Compton profiles for atomic and molecular systems, Adv. Quantum Chem. 10 (1977) 37-76. doi:10.1016/S0065-3276(08)60578-X.

[19] P. Benesch, V. H. Smith Jr., Radial momentum distributions for the $2 \mathrm{~S}$ ground state of the lithium atom, Chem. Phys. Lett. 5 (1970) 601-604. doi:10.1016/0009-2614(70)85058-8.

[20] P. Benesch, V. H. Smith Jr., Natural orbitals in momentum space and correlated radial momentum distributions: I. The 1S ground state of $\mathrm{Li}+$, Int. J. Quantum Chem. 5 (1971) 131-138. doi:10.1002/qua. 560050712 .

[21] P. Benesch, V. H. Smith Jr., Wave Mechanics the First Fifty Years, Butterworth, London, (1973) 357.

[22] A. Messiah, Quantum Mechanics, North-Holland Publ., Amsterdam, (1967) Vol I.

[23] P. Linz, A method for computing Bessel function integrals, Math. Comput. 26 (1972) 509-513. doi:10.1090/ S0025-5718-1972-0303687-8.

[24] J. D. Talman, Numerical Fourier and Bessel transforms in logarithmic variables, J. Comput. Phys. 29 (1978) 35-48. doi:10.1016/ 0021-9991 (78) 90107-9.

[25] S. M. Candel, An algorithm for the Fourier-Bessel transform, Comput. Phys. Commun. 23 (1981) 343-353. doi:10.1016/0010-4655(81) 90175-2. 
[26] A. V. Oppenheim, G. V. Frisk, D. R. Martinez, Computation of the Hankel transform using projections, J. Acoust. Soc. Am. 68 (1980) 523-529. doi:10.1121/1.384765.

[27] R. Piessens, Automatic computation of Bessel function integrals, Comput. Phys. Commun. 25 (1982) 289-295. doi:10.1016/ 0010-4655 (82) 90024-8.

[28] R. Piessens, M. Branders, Algorithm for the computation of Bessel function integrals, J. Comput. Appl. Math. 11 (1984) 119-137. doi: 10.1016/0377-0427(84)90037-2.

[29] M. Puoskari, A method for computing Bessel function integrals, J. Comput. Phys. 75 (1988) 334-344. doi:10.1016/0021-9991(88) 90116-7.

[30] J. D. Secada, Numerical evaluation of the Hankel transform, Comput. Phys. Commun. 116 (1999) 278-294. doi:10.1016/S0010-4655(98) 00108-8.

[31] L. Knockaert, Fast Hankel transform by fast sine and cosine transforms: The Mellin connection, IEEE Trans. Signal Proc. 48 (2000) 1695-1701. doi:10.1109/78.845927.

[32] M. Guizar-Sicairos, J. C. Gutiérrez-Vega, Computation of quasi-discrete Hankel transforms of integer order for propagating optical wave fields, J. Opt. Soc. Am. A 21 (2000) 53-58. doi:10.1364/JOSAA.21.000053.

[33] V. K. Singh, O. P. Singh, R. K. Pandey, Numerical evaluation of the Hankel transform by using linear Legendre multi-wavelets, Comput. Phys. Commun. 179 (2008) 424-429. doi:10.1016/j.cpc.2008.04. 006.

[34] V. K. Singh, O. P. Singh, R. K. Pandey, Efficient algorithms to compute Hankel transforms using wavelets, Comput. Phys. Commun. 179 (2008) 812-818. doi:10.1016/j.cpc.2008.07.005.

[35] J. L. Luna, H. H. Corzo, R. P. Sagar, Numerical evaluation of Bessel function integrals for functions with exponential dependence, Rev. Mex. Fís. E 59 (2013) 115-121.

[36] R. Wong, Quadrature formulas for oscillatory integral transforms, Numer. Math. 39 (1982) 351-360. doi:10.1007/BF01407868. 
[37] N. N. Lebedev, Special Functions and Their Applications, Dover Publications Inc., New York, (1972) Ch. 4.

[38] M. Abramowitz, Handbook of Mathematical Functions, National Bureau of Standards Applied Mathematics Series - 55, USA, (1972) Ch. 9 y Ch. 10.

[39] G. B. Arfken, Mathematical Methods for Physicists, Elsevier, USA, (2013) Ch. 11.

[40] W. W. Bell, Special Functions for Scientists and Engineers, D. Van Nostrand Company, London, (1968) Ch. 4.

[41] P. Linz, Theoretical Numerical Analysis, John Wiley \& Sons, Inc., USA, (1976) Ch. 3.

[42] W. Gautschi, Numerical Analysis an Introduction, Birkhäuser, Boston, (1997) Ch. 3.

[43] J. Stoer, R. Bulirsch, Introduction to Numerical Analysis, Springer-Verlag Inc., New York, (1993) Ch. 3.

[44] T. S. Chihara, An Introduction to Orthogonal Polynomials, Dover Publications Inc., New York, (2011) Ch. 1, Ch. 4 y Ch. 6.

[45] A. Asheim, D. Huybrechs, Complex Gaussian quadrature for oscillatory integral transforms, IMA J. Numer. Anal. 33 (2013) 1322-1341. doi: 10.1093/imanum/drs060.

[46] V. Dolotin, A. Morozov, Introduction to Non-linear Algebra, World Scientific Publishing Co. Pte. Ltd., (2007) Ch. 4.

[47] K. Sainath, F. L. Teixeira, Tensor Green's function evaluation in arbitrarily anisotropic, layered media using complex-plane Gauss-Laguerre quadrature, Phys. Rev. E 89 (053303) (2014) 1-27. doi:10.1103/PhysRevE. 89.053303.

[48] R. P. Sagar, H. Schmider, V. H. Smith Jr., Evaluation of Fourier transforms by Gauss-Laguerre quadratures, J. Phys. A 25 (1) (1992) 189-195. doi:10.1088/0305-4470/25/1/023.

[49] K. T. R. Davies, Complex-plane methods for evaluating highly oscillatory integrals in nuclear physics. II, J. Phys. G: Nucl. Phys. 14 (7) (1988) 973-993. doi:10.1088/0305-4616/14/7/015. 
[50] W. Gautschi, On generating orthogonal polynomials, SIAM J. Sci. Stat. Comput. 3 (1982) 289-317. doi:10.1137/0903018.

[51] W. Gautschi, Orthogonal Polynomials: Computation and Approximation, Oxford University Press, London, (2004).

[52] W. Gautschi, Computation of Bessel and Airy functions and of related Gaussian quadrature formulae, BIT 42 (2002) 110-118. doi:10.1023/ A : 1021974203359 .

[53] B. Zaslow, M. E. Zandler, Two-dimensional analog to the hydrogen atom, Am. J. Phys. 35 (1967) 1118-1119. doi:10.1119/1.1973790.

[54] H. N. Nunẽz, C. A. Vargas, A. L. Salas, The one-dimensional hydrogen atom in momentum representation, Eur. J. Phys. 8 (1987) 189-193.

[55] X. L. Yang, S. H. Guo, F. T. Chan, Analytic solution of a two-dimensional hydrogen atom. I. Nonrelativistic theory, Phys. Rev. A 43 (1991) 1187-1196. doi:10.1103/PhysRevA.43.1186.

[56] J. Avery, Hyperspherical Harmonics and Generalized Sturmians, Kluwer Academic Publishers, USA, (2002) Ch. 2.

[57] V. Aquilanti, S. Cavalli, C. Coletti, The D-dimensional hydrogen atom: hyperspherical harmonics as momentum space orbitals and alternative Sturmian basis sets, Chem. Phys. 214 (1997) 1-13. doi:10.1016/ S0301-0104 (96)00310-2.

[58] A. Erdélyi, Tables of integral transforms, McGraw-Hill Book Company Inc., USA, (1954) Vol. II. Ch. 8.

[59] R. J. Yáñez, W. V. Assche, J. S. Dehesa, Position and momentum information entropies of the D-dimensional harmonic oscillator and hydrogen atom, Phys. Rev. A 50 (1994) 3065-3079. doi:10.1103/ PhysRevA.50.3065.

[60] P. W. Thulstrup, Smooth interpolation, Fourier transformation, and two-center overlap integrals for numerical atomic orbitals, Int. J. Quantum Chem. 9 (1975) 789-795. doi:10.1002/qua.560090504.

[61] A. J. Thakkar, V. H. Smith Jr., A strategy for the numerical evaluation of Fourier sine and cosine transforms to controlled accuracy, Comput. Phys. Commun. 10 (1975) 73-79. doi:10.1016/0010-4655(75) 90073-9. 
[62] E. Clementi, Tables of atomic functions, IBM J. 9 (1965) 87-89. doi: $10.1147 /$ JRD . 1965.5392159.

[63] P. E. Cade, A. C. Wahl, Hartree-Fock-Roothaan wavefunctions for diatomic molecules: II. First-row homonuclear systems A2, A2-, A2+ and A2*, At. Data Nucl. Data 13 (1974) 339-389. doi:10.1016/ 0092-640X (74) 90006-0.

[64] M. Toyoda, T. Ozaki, Fast spherical Bessel transform via fast Fourier transform and recurrence formula, Comput. Phys. Comm., 181 (2010) 277-282. doi:10.1016/j.cpc.2009.09.020.

[65] H. L. Krall, O. Frink, A new class of orthogonal polynomials: the Bessel polynomials, Amer. Math. Soc. 130 (1949) 100-115.

[66] S. F. Boys, Electronic wave functions. I. A general method of calculation for the stationary states of any molecular system, Proc. R. Soc. London Ser. A 200 (1950) 542-554.

[67] R. McWeeny, Gaussian approximations, to wave functions, Nature. London, 166 (1950) 21-22. doi:10.1038/166021a0.

[68] F. E. Harris, Gaussian wave functions for polyatomic molecules, Rev. Mod. Phys., 35 (1963) 558-569. doi:10.1103/RevModPhys.35.558.

[69] D. A. McQuarrie, Quantum Chemistry, University Science Books, California, (2008) Ch. 9.

[70] T. Koga, K. Kanayama, S. Watanabe, A. J. Thakkar, Analytical Hartree-Fock wave functions subject to cusp and asymptotic constraints: He to $\mathrm{Xe}, \mathrm{Li}+$ to $\mathrm{Cs}+$, H- to I-, Int. J. Quantum Chem. 71 (1999) 491-497. doi:10.1002/(SICI) 1097-461X(1999) 71:6<491: : AID-QUA6>3 . 0 . CO;2-T.

[71] E. Clementi, C. Roetti, Roothaan-Hartree-Fock atomic wavefunctions: Basis functions and their coefficients for ground and certain excited states of neutral and ionized atoms, $\mathrm{Z}<54$, At. Data Nucl. Data 14 (1974) 177-478. doi:10.1016/S0092-640X (74)80016-1.

[72] M. Toyoda, T. Ozaki, Numerical evaluation of electron repulsion integrals for pseudoatomic orbitals and their derivatives, J. Chem. Phys. 130 (2009) 1-7. doi:10.1063/1.3082269. 
[73] H. Safouhi, Efficient and rapid numerical evaluation of the two-electron, four-center Coulomb integrals using nonlinear transformations and useful properties of sine and Bessel functions, J. Comp. Phys. 176 (2002) 1-19. doi:10.1006/jcph.2001.6925.

[74] F. R. Ornellas, The integral transform approach the electron-repulsion integral and the Hellium atom, J. Chem. Edu. 62 (1985) 378. doi: 10.1021/ed062p378.

[75] A. Iserles, S. P. Nørsett, Efficient quadrature of highly oscillatory integrals using derivatives, Proc. R. Soc. A. 461 (2005) 1383-1399. doi:10.1098/rspa.2004.1401.

[76] A. Iserles, S. P. Nørsett, On quadrature methods for highly oscillatory integrals and their implementation, BIT Numerical Mathematics 44 (2004) 755-772. doi:10.1007/s10543-004-5243-3.

[77] E. K. Ryu, S. P. Boyd, Extensions of Gauss quadrature via linear programming, Found. Comput. Math. 15 (2015) 953-971. doi : $10.1007 /$ s10208-014-9197-9.

[78] Mathematica, Version 10, Wolfram Research, Inc., Champaign, IL, USA, (2016). 